\title{
Augmenting the Detection of Verotoxin-Producing Escherichia coli Through Use of Fluorescently Labeled DNA Oligonucleotides
}

\author{
by \\ Dylan Fox-Altherr \\ A thesis submitted to the Faculty of Graduate and Postdoctoral Affairs in partial \\ fulfillment of the requirements for the degree of \\ Master of Science \\ in
}

Chemistry with Specialization in Chemical and Environmental Toxicology

Carleton University

Ottawa, Ontario

(C)2018

Dylan Fox-Altherr 


\begin{abstract}
Verotoxin-producing Escherichia coli (VTEC) present a significant risk of foodborne illness and severe patient outcomes. Isolation is laborious and time consuming due to the diversity of strains. Development of a differential agent would enhance rate and speed of isolation. Production of verotoxin (VT) is the only phenotypic trait exclusive to VTEC.

Aptamers are single-stranded oligonucleotides with high selectivity and affinity for their targets. A VT-targeting aptamer beacon could aid in isolation of VTEC. Proof of concept was demonstrated using an existing aptamer sequence, showing increased fluorescence in the presence of VT1a. Further characterization showed target specificity, but weaker signal contrast in complex media. Comparison of fluorophore-quencher pairs showed Texas Red and Black Hole Quenchers as optimal choices. Finally, a DNA substrate developed to detect VT enzyme activity produced weak fluorescent signal making it unlikely to aid in detection.
\end{abstract}




\section{Acknowledgements}

I would like to thank Health Canada and Carleton University for the opportunity to work on this project and for all the education, experiences, and support l've received over the past 7 years through Bachelor's, Master's, and co-op placements.

I would like to thank both Maria DeRosa and Alex Gill for all their supervision, advice, problem solving, and the expertise that they shared with me through the course of this project regarding aptamers and VTEC; refreshing beverages, all-dressed chips, and life; as well as the opportunity to work in both of their laboratories. Every time I thought there was a major problem they'd act like it was nothing. Their confidence and encouragement was of great benefit and I can't thank them enough.

I would like the thank Mary Rao and Robyn Kenwell for all the work behind the scenes to keep the VTEC lab running. All the weeks put into purifying toxin for me, the ups and downs of cloning VT expression vectors, all the piles of other work and family responsibilities on their plates is probably more than I could handle. ;P

I would like to thank Daniel Brumar for the hard work and effort he put into the project during the summer of 2016. Combined with his sense of humour it was an enjoyable summer while we weren't worrying about him. ;P

I would like to thank Nadine Frost for all her assistance in learning how to use the instruments at Carleton as well as all the assistance in DNA synthesis and purification. I would like to thank Erin McConnell for all her wisdom and constant positive attitude. I would like to thank Christopher Mattice who was a wealth of knowledge whenever I had questions about chemical reactions and problem solving. 
And Emily Mastronardi who was a great TA, PhD senpai, and whose encouragement led to joining the DeRosa lab in the first place! :P

I would also like to thank each of the other members of the DeRosa lab for the various ways they have assisted throughout this last few years and for providing such a friendly, cooperative environment to do research in and compare Pokédexes.

I would also like to thank Laura Arvaj, Annie Jung, Luke Dick, Michael Liu, and Allen Garcia for the various ways they kept me sane throughout the past couple years. I'd like to thank the various friends who have shown excitement for my project when explaining it either in detail or passing when I may or may not have had excitement for it. I would like to thank my parents for their patience, trust, and support in everything.

Finally, I would like to thank Ruth Hill-Lapensee and Chantelle Gravelle for all their assistance in registration and all the logistic details.

I'd also like to say that none of this would have been possible aside from the endless graces that God has shown me over the years. I still remember how deeply impacted by the reality of grace I was when I showed up for my second year biochem lab 30 minutes late with a lab report I had worked on almost exclusively that morning and had my laptop freeze as I was finishing (after not saving for hours). Or the time I literally walked into an Organic midterm having not studied at all and ending up with an A+ for a final grade. Or the time I was blessed to go to Haiti to help rebuild an orphanage and I also had a formal lab report due the following week. My coordinator gave me an extension when I asked. Or the time I had procrastinated applying for co-op jobs and just didn't have the time so I'd either have to devote so much extra time to it 
or just not do co-op. And while praying about it with a friend I received an email with a job interview for a job I didn't realize I had applied for (learned about the things I needed for the interview in Plant Physiology class the hour before, and ended up getting that job). Or when my plans to intern as a missionary for a couple years fell through and I thought I had no options left. Within a couple weeks I was accepted for my M.Sc. And most impactfully while TAing being able to better grasp how God loves me through my relationship with all my students. I literally told them all to just slap a conclusion on their report and go to sleep so they' $d$ be able to do their lab exam. Many did not follow this simple instruction. One guy even pulled an all-nighter and forgot an equation he needed which was in his notebook (it was an open notebook lab exam) causing him to do so badly on it. And even though they regularly made mistakes and didn't listen to me I still cared for them and wanted them to succeed. Seeing God's provision for me this last September and the awesome friends and coworkers He's given me. Even to this month as it felt like my life was literally falling apart He has kept me and got me through it all. Through the cross and the resurrection of Jesus even though I make endless mistakes and choose so many things that aren't God and what He'd have for me, even though He has told me again and again, He's always there to pick me up and love and forgive and move forward. Honestly, I continue to marvel at the graces extended to me. By the grace of God, I am who I am. He will love me when I can't. He will use me to do greater things than I can imagine. In His timing. He will bring this incomplete composition to completion. This is Amazing Grace. So much more than I could ask or deserve. 


\section{Table of Contents}

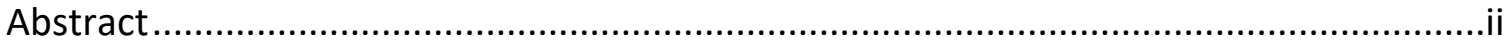

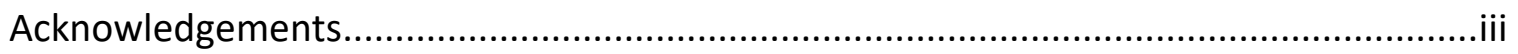

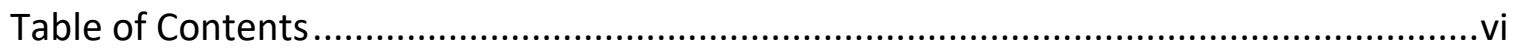

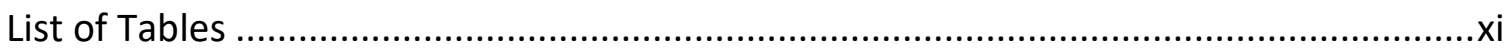

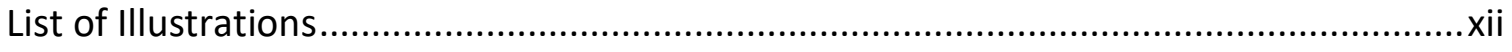

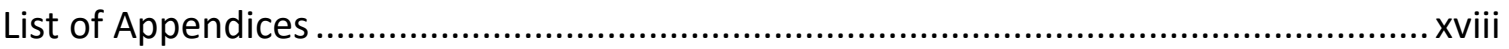

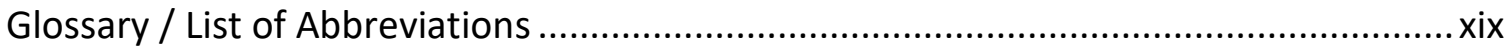

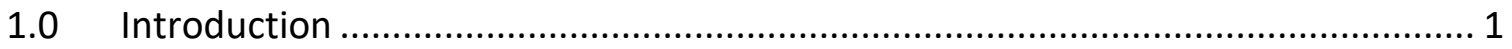

1.1 Verotoxin (Shiga Toxin, Shiga-like Toxin; VT) .............................................. 1

1.1.1 General Information and Structure ........................................................... 1

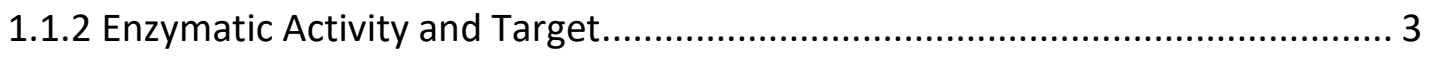

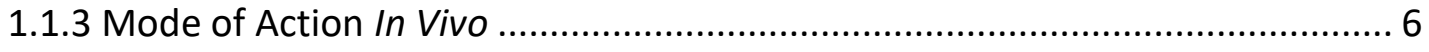

1.1.3.1 B Subunit: Receptor Binding, Holotoxin Formation, and Uptake ............. 6

1.1.3.2 A Subunit: Retrograde Transport and Ribosome Inactivation ................. 7

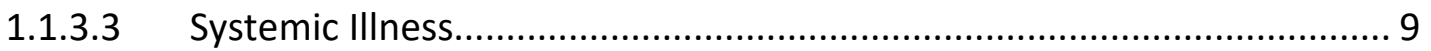

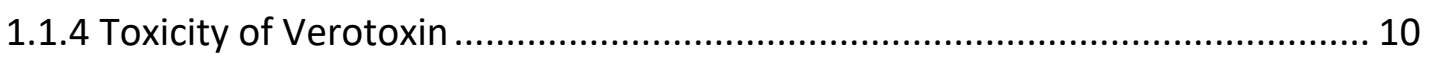

1.1.5 Verotoxin Subtypes and Disease Association .............................................. 13

1.1.6 Factors Implicated in Higher Disease Severity for VT2 ............................... 16

1.2 Verotoxin-producing Escherichia coli (VTEC) ................................................ 18

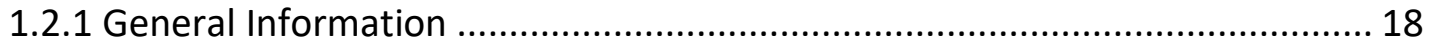

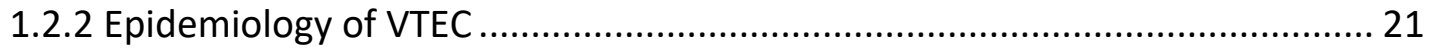

1.2.3 Verotoxin Production, Expression, and Virulence Factors ............................ 25

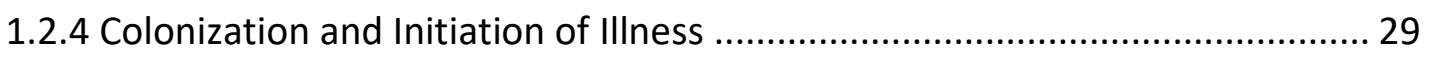

1.2.5 VTEC Associated IIIness and Treatment.................................................... 30

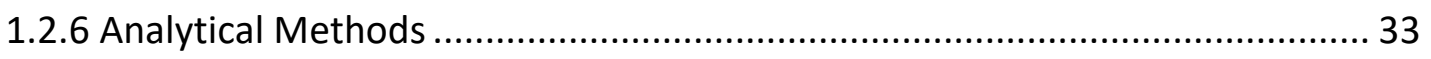

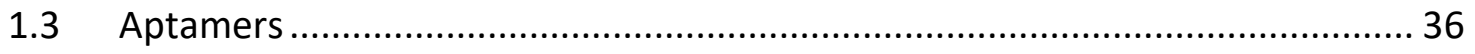

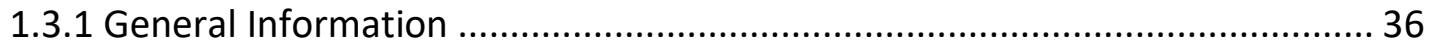

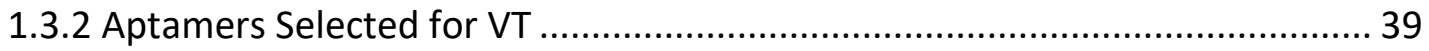

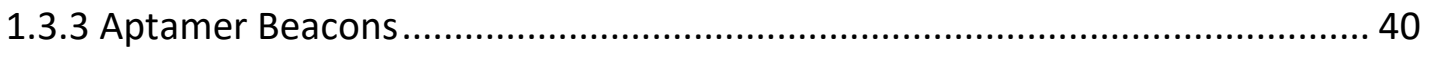

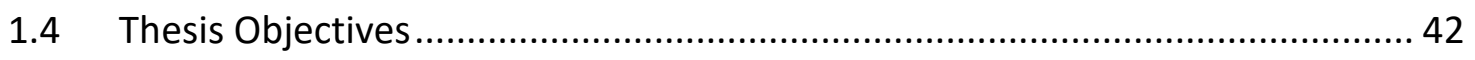




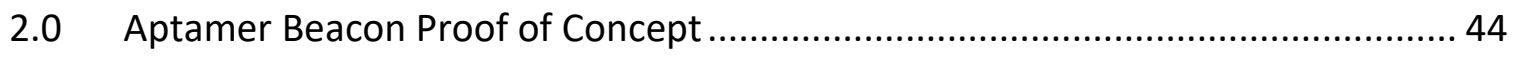

2.1 Introduction........................................................................................... 44

2.1.1 The Benefit of a VT Aptamer Beacon.......................................................... 44

2.1.2 Fluorescence Measurement ..................................................................... 45

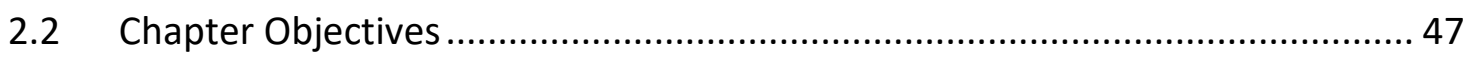

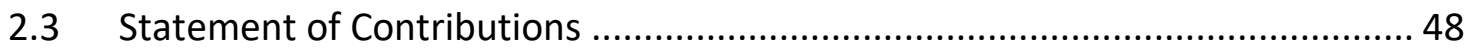

2.4 Materials and Methods ........................................................................... 48

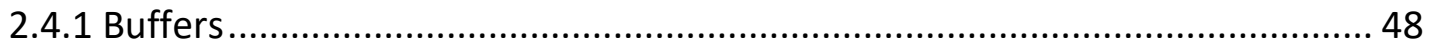

2.4.2 Preliminary structural analysis and aptamer beacon design ........................... 48

2.4.3 DNA Synthesis, purification, and quantification ........................................... 49

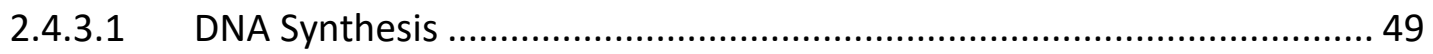

2.4.3.2 Sample Clean-up and Quantification ................................................. 50

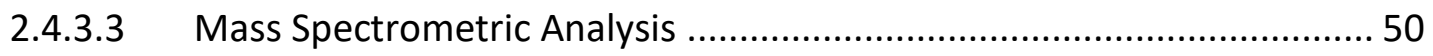

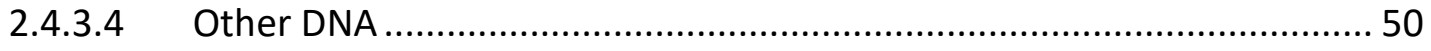

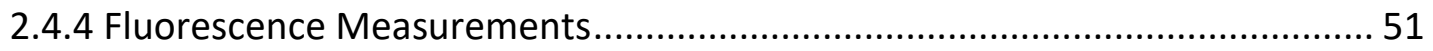

2.4.5 Linear Fluorescence Range .......................................................................... 51

2.4.6 Effect of Temperature and Melting Temperature Determination .................. 52

2.4.7 Effect of Reannealing Media..................................................................... 52

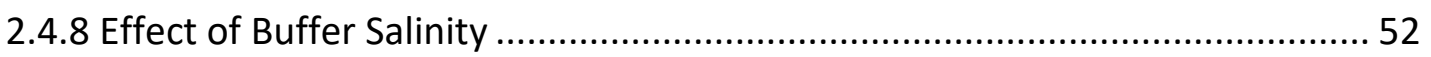

2.4.9 Effect of Complement Length ................................................................... 53

2.4.10 Effect of Toxin on Probe Fluorescence ........................................................ 53

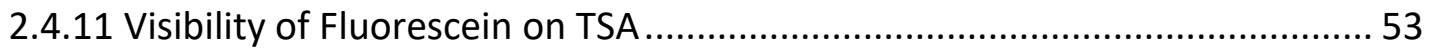

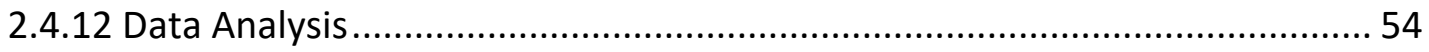

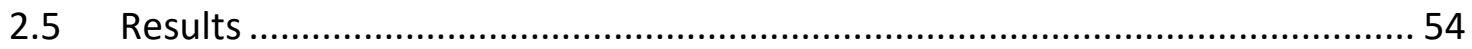

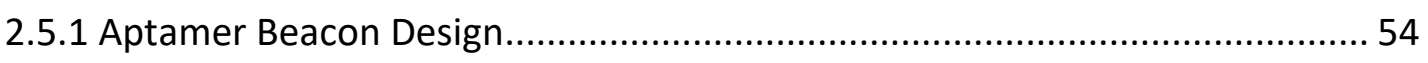

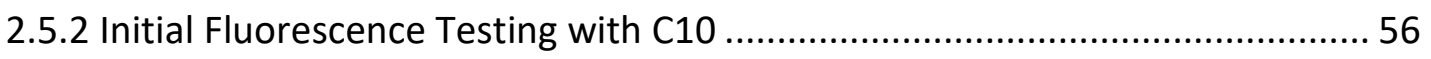

2.5.3 Melting Temperature Determination and Effect of Temperature .................. 57

2.5.4 Effect of Reannealing Media.......................................................................... 58

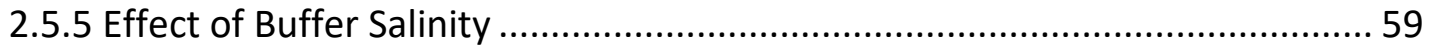

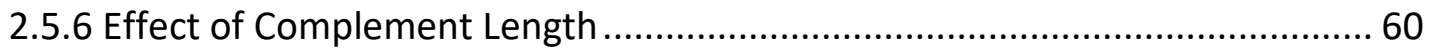

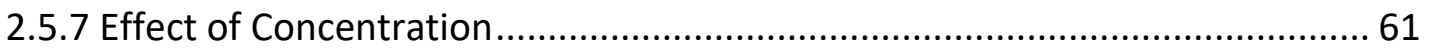

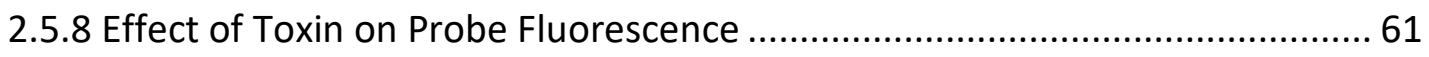

2.5.9 Visibility of Fluorescein-Tagged Sequences on TSA ......................................... 63 


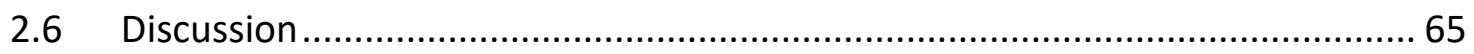

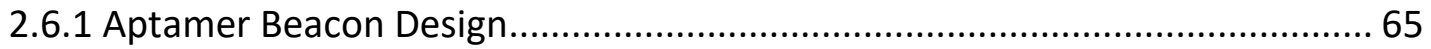

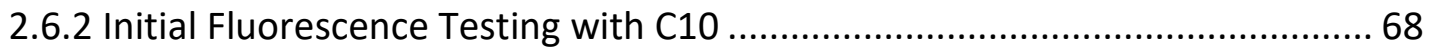

2.6.3 Melting Temperature Determination and Effect of Temperature .................. 70

2.6.4 Effect of Reannealing Media....................................................................... 71

2.6.5 Effect of Buffer Salinity .......................................................................... 72

2.6.6 Effect of Complement Length ......................................................................... 73

2.6.7 Effect of Concentration............................................................................. 74

2.6.8 Effect of Toxin on Probe Fluorescence ...................................................... 75

2.6.9 Visibility of Fluorescein-Tagged Sequences on TSA ...................................... 78

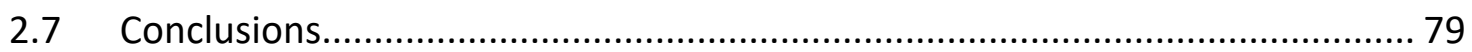

3.0 Further Characterizing the Aptamer Beacon ................................................. 81

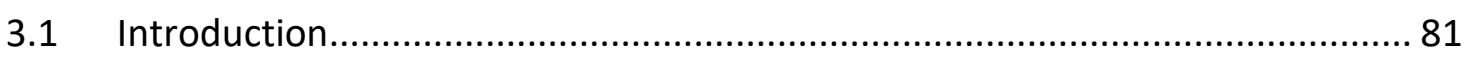

3.1.1 Characterization of Aptamer Beacons ......................................................... 81

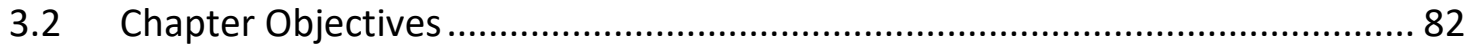

3.3 Statement of Contributions ……………................................................... 82

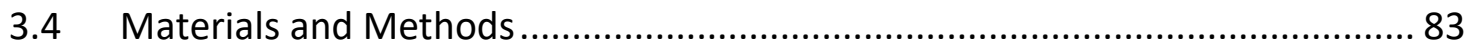

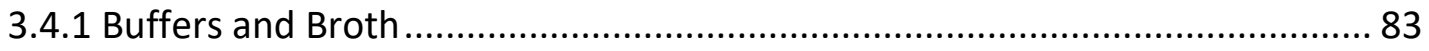

3.4.2 DNA Synthesis, purification, and quantification........................................... 83

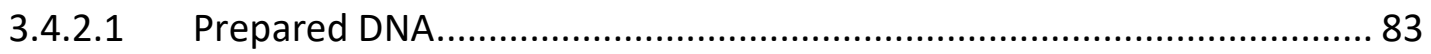

3.4.3 Fluorescent Measurements .......................................................................... 83

3.4.4 Linear Range of Fluorescence ....................................................................... 84

3.4.5 Determination of Potential Fluorescence................................................... 84

3.4.6 Molecular Beacon Potential in TSB ............................................................. 84

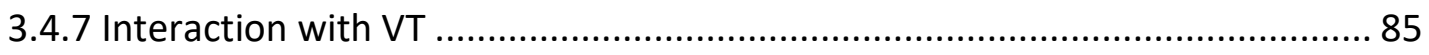

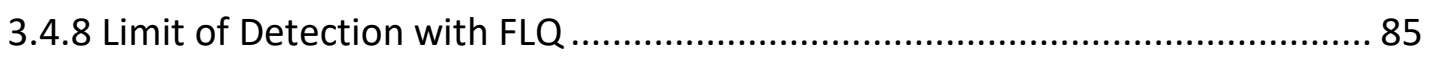

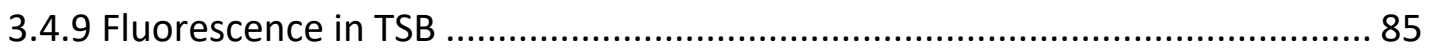

3.4.10 Fluorescence with Crude Toxin................................................................... 86

3.4.11 Effect of non-specific protein quenching..................................................... 87

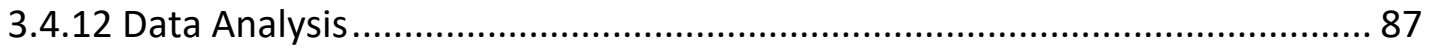

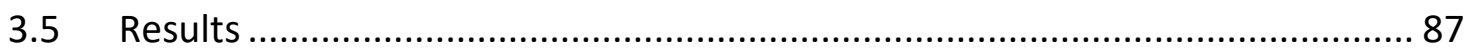

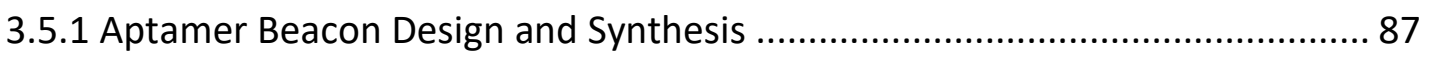

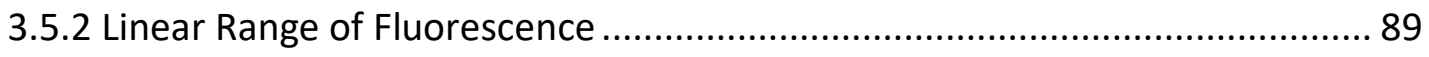




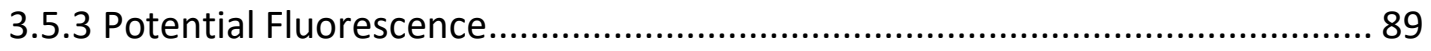

3.5.4 Molecular Beacon Potential in TSB .......................................................... 90

3.5.5 Fluorescence in the Presence of Toxin ...................................................... 91

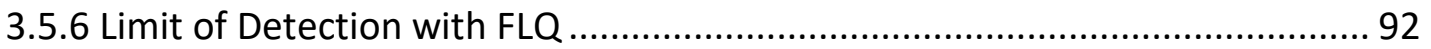

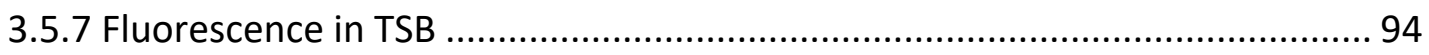

3.5.8 Fluorescent Signal Induced with Crude Toxin.................................................. 96

3.5.9 Effect of Non-specific Protein Quenching..................................................... 98

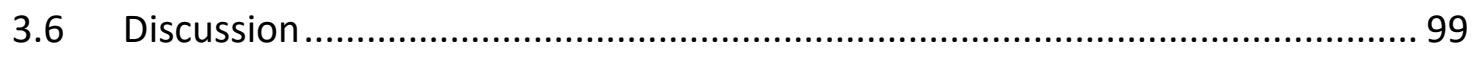

3.6.1 Aptamer Beacon Design and Synthesis .......................................................... 99

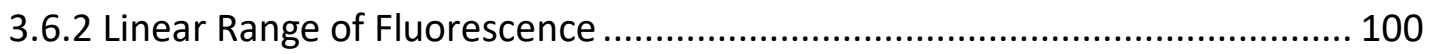

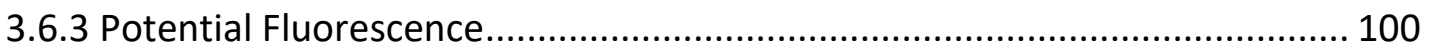

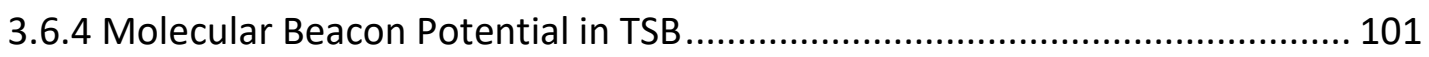

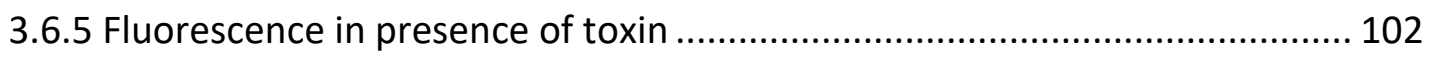

3.6.6 Limit of Detection with FLQ ..................................................................... 104

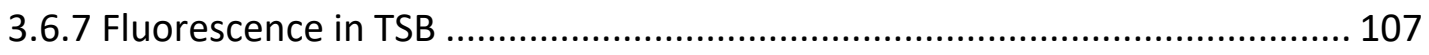

3.6.8 Effect of Crude Toxin Prep ......................................................................... 109

3.6.9 Effect of Non-specific Protein Quenching................................................... 110

3.7 Conclusions / Future Directions ……………........................................... 111

4.0 Comparing Fluorophore / Quencher Pairs.................................................... 113

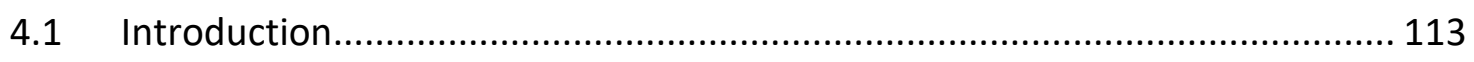

4.1.1 Fluorophore / Quencher Pairs .............................................................. 113

4.1.2 Selecting New Pairs .............................................................................. 113

4.2 Chapter Objectives .............................................................................. 114

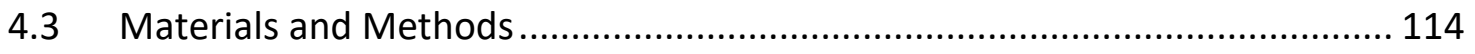

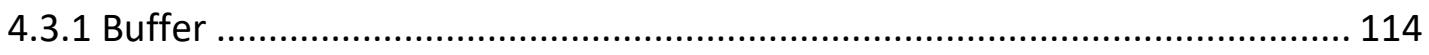

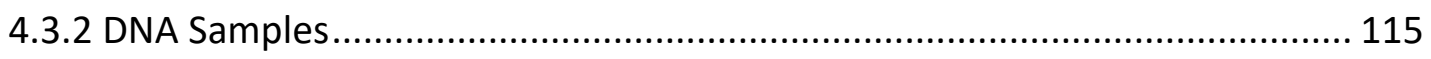

4.3.3 Fluorescent Measurements ................................................................. 116

4.3.4 Determination of Linear Fluorescent Range .................................................. 117

4.3.5 Determination of Potential Fluorescence................................................... 117

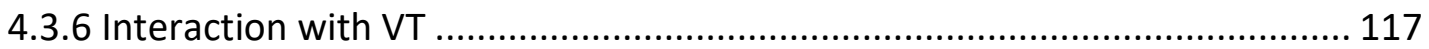

4.3.7 Comparison of Visible Limit of Detection .................................................... 118

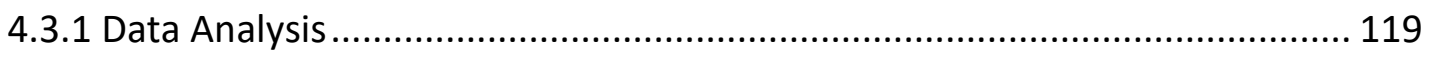

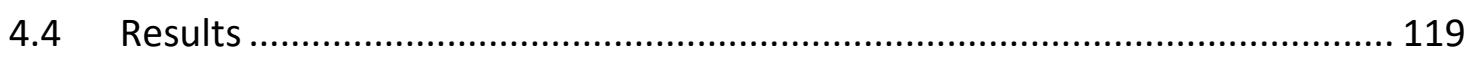




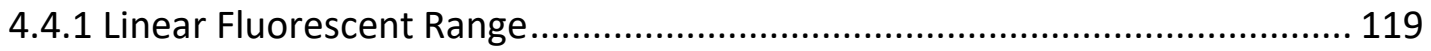

4.4.2 Potential Fluorescence with Complete Complement ................................ 120

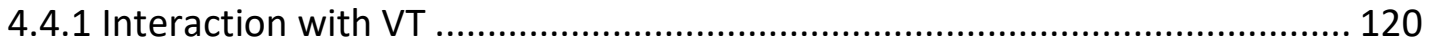

4.4.2 Comparison of Visible Limit of Detection ................................................ 124

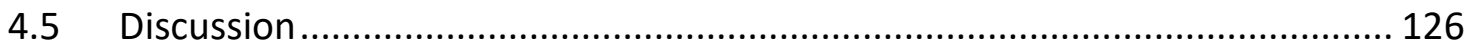

4.5.1 Linear Fluorescent Range .................................................................. 126

4.5.2 Potential Fluorescence with Complete Complement.................................. 129

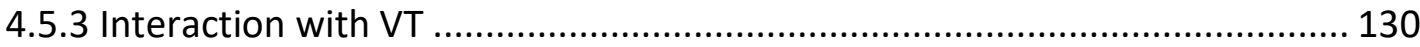

4.5.4 Comparison of Visible Limit of Detection ................................................ 131

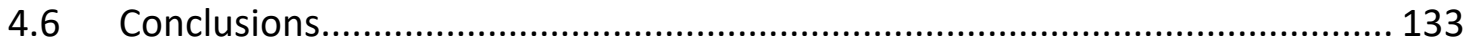

5.0 PolyA DNA Substrate for Enzymatic Activity Differentiation............................. 134

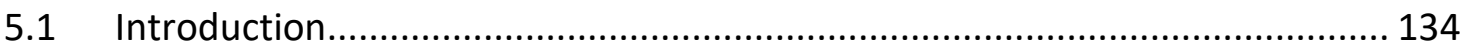

5.1.1 Use of VT Enzyme Activity as a Detection Method for VT2 ........................ 134

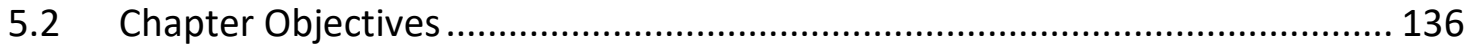

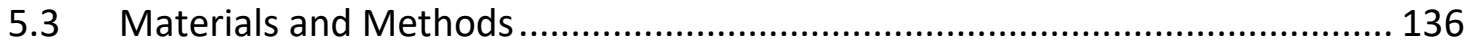

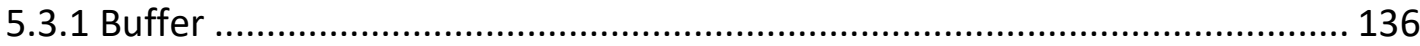

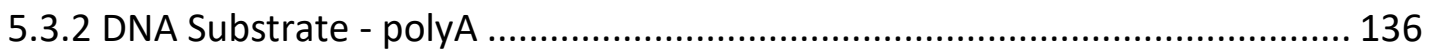

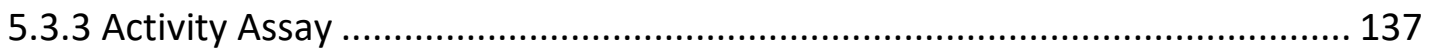

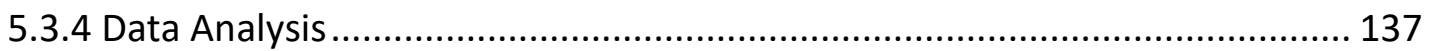

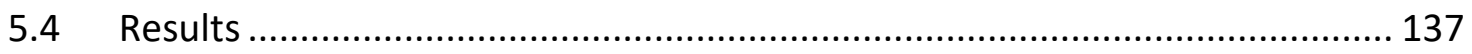

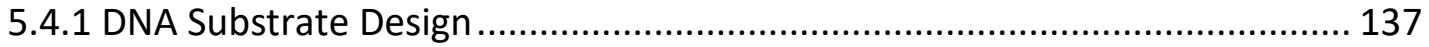

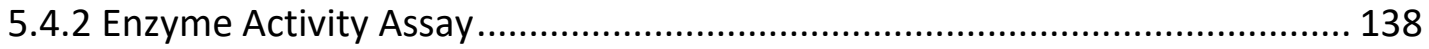

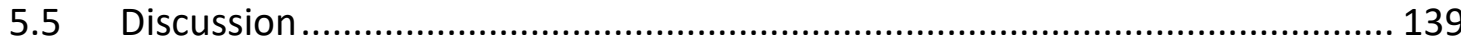

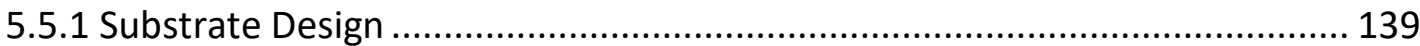

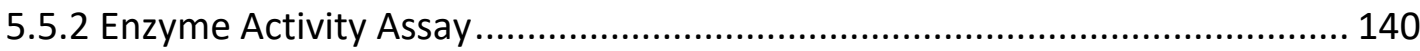

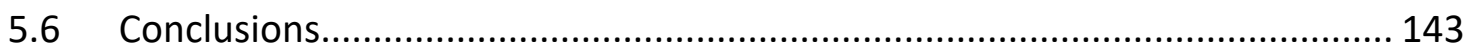

6.0 Contributions to knowledge and future studies .......................................... 144

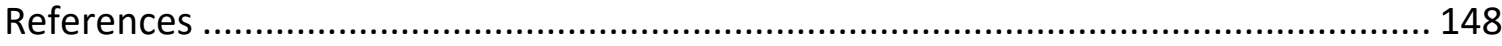

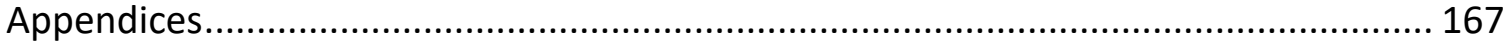




\section{List of Tables}

Table 1 - Summary of select VT or Shiga Toxin toxicity trials .................................... 11

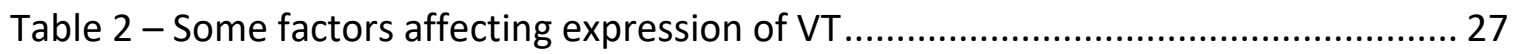

Table 3 - Aptamer sequences selected for verotoxin ............................................. 40

Table 4 - Buffers used in Chapter 2.0 Aptamer Beacon Proof of Concept ..................... 48

Table 5 - Buffers and broth used in Chapter 3.0 - Further Characterizing the Aptamer

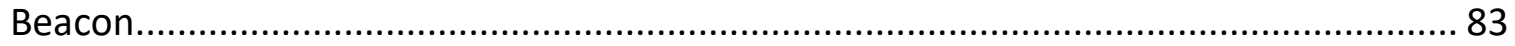

Table 6 - Characteristics of fluorophores and quenchers compared in this study ....... 115

Table 7 - Comparison of unquenched signal loss over 1 hour in buffer ...................... 131 


\section{List of Illustrations}

Figure 1 - Verotoxin holotoxin structure 3D model. A) A subunit (silver) interacting noncovalently with the $B$ subunit pentamer (gold) via the A2 chain. A red arrow indicates the cleavable loop. B) The five B subunits coloured differently. Model produced by JSmol web application from Protein Data Bank entry 1DMO: SHIGA TOXIN (Fraser et al., 1994).

Figure 2 - The structure of the SRL region targeted by verotoxin. Left) the stem loop structure of SRL portion of the large ribosomal rRNA subunit; Adenine-4324 indicated by an arrow. Right) the structure of deoxyadenosine monophosphate with the cleavage site indicated by thick red line. SRL model produced by NGL web application from Protein Data Bank entry 430D (Correll et al., 1998); Chemical structure produced with ChemDoodle web application.

Figure 3 - Generalized overview of the SELEX process used to select aptamers for specific targets

Figure 4-Schematic diagram of the modified aptamer beacon with no fluorescence (middle) and interaction with complement DNA (left) and verotoxin (right) resulting in a conformational shift, producing an increased fluorescent emission.

Figure 5 - Dabcyl CPG (1-Dimethoxytrityloxy-3-[O-(N-4'-carboxy-4-(dimethylamino)azobenzene)-3-aminopropyl)]-propyl-2-O-succinoyl-long chain alkylamino-CPG)

Figure 6-5' fluorescein phosphoramidite (6-(3',6'-dipivaloylfluoresceinyl-6-

carboxamido)-hexyl-1-O-(2-cyanoethyl)-(N,N-diisopropyl)-phosphoramidite)

Figure 7 - Secondary structure of Fan et al.'s 40 base forward (+) verotoxin DNA aptamer sequence predicted with RNAstructure software A) unmodified; B) with 10 complementary bases at the $3^{\prime}$ end (A10); and C) with 15 complementary bases (A15) (Reuter and Matthews, 2010).....

Figure 8 - Secondary structure for a 15-base DNA complement (5' - TGA ATG CTA GTT ACC $-3^{\prime}$ ) using RNAstructure (left) and Mfold (right) software (Reuter and Matthews, 2010; Zuker, 2003).

Figure 9 - Fluorescence intensity for fluorescein / dabcyl labeled aptamer beacon with 10 complementary bases (A10) and 15 complementary bases (A15) at $1 \mu \mathrm{M}$ with increasing of beacon: complement ratios (1:0,1:1, 1:2, and 1:4) over an hour in TEB buffer. DNA complement was 10 bases long (C10). Fluorescent control values were about $85 \times 10^{4}$ RFUs. Data points represent the average of two wells (error bars show SD). Ex. $495 \pm 10 \mathrm{~nm}$, Em. $528 \pm 20 \mathrm{~nm}$.

Figure 10 - Melting temperature curves for aptamer beacons with 10 complementary 
bases (A10) (left) and 15 complementary bases (A15) (right) prepared using $260 \mathrm{~nm}$ spectrophotometer absorbance readings and SigmaPlot software.

Figure 11 - Fluorescence intensity of fluorescent control DNA (FC) and fluorescein / dabcyl labeled aptamer beacon with 10 complementary bases (A10) at $1 \mu \mathrm{M}$ in TEB buffer. About 40 minutes passed while heating to $60^{\circ} \mathrm{C}$. Data points represent the average of $3(\mathrm{FC})$ or 8 wells (A10) with error bars showing SD. Ex. $495 \pm 10 \mathrm{~nm}, \mathrm{Em} .528 \pm$ $20 \mathrm{~nm}$.

Figure 12 - Fluorescence intensity effects of refolding in TEB buffer (B) of fluorescein / dabcyl labeled aptamer beacon with 10 complementary bases (A10) $(1 \mu \mathrm{M})$ alone and in the presence of $\mathrm{C} 10(4 \mu \mathrm{M})$. fluorescent control DNA $(\mathrm{FC})$ had a value of about $30 \times 10^{5}$ RFUs. Data points are the average of 3 samples. Error bars show SD. Ex. $495 \pm 10 \mathrm{~nm}$, Em. $528 \pm 20 \mathrm{~nm}$. 59

Figure 13 - Comparison of fluorescence intensity of fluorescein / dabcyl labeled aptamer beacon with 10 complementary bases (A10) $(1 \mu \mathrm{M})$ in low or high salt buffer with or without C10 $(4 \mu \mathrm{M})$. Buffer had $20 \mathrm{mM}(\mathrm{TEB})$ or $1 \mathrm{M}$ (TES) of $\mathrm{NaCl} / \mathrm{KCl}$. Data points are the average of 4 wells. Error bars show SD. Fluorescent control values were about $34 \mathrm{x}$ $10^{5}$ RFUs. Ex. $495 \pm 10 \mathrm{~nm}$, Em. $528 \pm 20 \mathrm{~nm}$.

Figure 14 - Fluorescence intensity of fluorescein / dabcyl labeled aptamer beacon with 10 complementary bases (A10) $(1 \mu \mathrm{M})$ in the presence of $\mathrm{C} 10$ or 15 base complement (C15) $(1 \mu \mathrm{M})$ in TEB (B) and TES (S). Fluorescent control values were about $33 \times 10^{5}$ RFUs. Data points are the average of 4 wells (B and S), 5 wells (S-C10), and 6 wells (others). Error bars represent SD. Ex. $495 \pm 10 \mathrm{~nm}, \mathrm{Em} .528 \pm 20 \mathrm{~nm}$.

Figure 15 - Fluorescence intensity of fluorescein / dabcyl labeled aptamer beacon with 10 complementary bases (A10) $(50 \mathrm{nM})$ in the presence of $\mathrm{C} 10$ or 15 base complement (C15) (50 nM) in TEB (B) and TES (S). Fluorescent control values were about $12 \times 10^{4}$ RFUs. Data points are the average of 6 wells except $B$ and $S$ which had 4 wells. Error bars represent SD. Ex. $495 \pm 10 \mathrm{~nm}$, Em. $528 \pm 20 \mathrm{~nm}$.

Figure 16 - Fluorescence intensity results for fluorescein / dabcyl labeled aptamer beacon with 10 complementary bases (A10) ( $50 \mathrm{nM}$ ) testing at a 1:1 ratio with C15, VT1a, and VT2a in TES buffer. A) results over 60 minutes; B) Results at 60 minutes. * and $* *$ values significantly different from background $p=0.05$ and $p=0.01$, respectively. Fluorescent control DNA (FC) values were about $13 \times 10^{4} \mathrm{RFUs}$. Data points are the average of 16 wells (A10 and C15) and 4 wells (VT1 and VT2) over two replicates. Error bars represent SD. Ex. $495 \pm 10 \mathrm{~nm}$, Em. $528 \pm 20 \mathrm{~nm}$. 64

Figure 17 - Fluorescence intensity results for fluorescein / dabcyl labeled aptamer beacon with 10 complementary bases (A15) ( $50 \mathrm{nM}$ ) testing at a 1:1 ratio with $\mathrm{C} 15$, VT1a, and VT2a in TES buffer. A) results over 60 minutes; B) Results at 60 minutes. * and $* *$ values significantly different from background $p=0.05$ and $p=0.01$, respectively. 
Fluorescent control DNA (FC) values were about $13 \times 10^{4}$ RFUs. Data points are the average of 16 wells (A10), 14 wells (C15), and 4 wells (VT1 and VT2) over two replicates. Error bars represent SD. Ex. $495 \pm 10 \mathrm{~nm}$, Em. $528 \pm 20 \mathrm{~nm}$.

Figure 18 - Solutions of fluorescent control DNA (FC) at concentrations of 40, 30, 20, 10 $\mu \mathrm{M}$ under UV lamp. Image enhanced. 65

Figure 19 - Handheld UV lamp-illuminated TSA plate spotted with six spots of $10 \mu \mathrm{L}$ fluorescein labeled DNA at each of 50, 40, 30, 20, and $10 \mu \mathrm{M}$. Image enhanced to reflect what was seen by eye. Red circles highlight examples of visibly fluorescent fluorescent control DNA (FC) spots.

Figure 20 - Handheld UV lamp-illuminated TSA plates spotted with $10 \mu \mathrm{L}$ of fluorescein / dabcyl labeled aptamer beacon with 10 complementary bases (A10) (50 $\mu \mathrm{M})$ and C15 $(100 \mu \mathrm{M})$, together, separately, and fluorescent control DNA (FC) $(30 \mu \mathrm{M})$. Left: A10 spotted first; right: C15 spotted first. Images enhanced to reflect what was seen by eye. Red circles highlight a visibly fluorescent control spot. 66

Figure 21 - Most probable secondary structure for the DNA sequence of the aptamer beacon with 13 complementary bases using RNAstructure (left) and Mfold (right) software (Reuter and Matthews, 2010; Zuker, 2003).

Figure 22 - Most probable secondary structure for complete complement DNA (CC) using RNAstructure (left) and Mfold (right) software (Reuter and Matthews, 2010; Zuker, 2003). 88

Figure 23 - Fluorescent control (FLX) calibration curve in TES buffer. Data points are the average of 9 wells over 3 replicates. Error bars represent 95\% Cl. Ex. $495 \pm 10$ nm, Em. $528 \pm 20 \mathrm{~nm}$.

Figure 24 - Comparison of the fluorescence of fluorescein / dabcyl labeled aptamer beacon (FLQ) in the presence of equimolar complete complement DNA (CC) compared to fluorescein labeled aptamer (FLX) and FLQ alone in TES buffer. Data points are the average of 9 wells over 3 replicates. Error bars represent $95 \% \mathrm{Cl}$. Ex. $495 \pm 10 \mathrm{~nm}$, Em. $528 \pm 20 \mathrm{~nm}$.

Figure 25 - Fluorescence of fluorescein / dabcyl labeled aptamer beacon (FLQ) induced by binding complete complement DNA (CC) (10 nM) in TES buffer and TSB media over time compared to fluorescein labeled aptamer (FLX). Data points represent the average of 3 samples, except for B-FLQ/CC (2 samples). Error bars represent SD. Ex. $495 \pm 10 \mathrm{~nm}$, Em. $528 \pm 20 \mathrm{~nm}$. 91

Figure 26 - Fluorescence of fluorescein / dabcyl labeled aptamer beacon (FLQ) (10 nM) with equimolar VT1a or VT2a over time in TES buffer. A) Fluorescence intensity of all samples; B) fluorescence intensity excluding fluorescein labeled aptamer (FLX). Data 
points are the average of 12 wells over 4 replicates, except for VT2 ( 9 over 3 ). Error bars represent $95 \% \mathrm{Cl}$. Ex. $495 \pm 10 \mathrm{~nm}$, Em. $528 \pm 20 \mathrm{~nm}$. 92

Figure 27 - Fluorescence of fluorescein / dabcyl labeled aptamer beacon (FLQ) (10 nM) after 70 minutes with varying VT1 concentrations in TES buffer. Fluorescein labeled aptamer (FLX) values ( $10 \mathrm{nM}$ ) were around $17 \times 10^{3}$ RFUs. Data points represent the average of 6 wells from replicates 1 and 2. Error bars shown are SD. Ex. $495 \pm 10 \mathrm{~nm}$, Em. $528 \pm 20 \mathrm{~nm}$. * and $* *$ values significantly different from $0 \mathrm{nM}$ VT $p=0.05$ and $p=$ 0.01 , respectively.

Figure 28 - Change in fluorescence signal of fluorescein / dabcyl labeled aptamer beacon (FLQ) in the presence of varying concentrations of VT1 mixed immediately before reading in TES buffer. Data points are the average of 9 samples over 3 trials. Ex. $495 \pm 10$ nm, Em. $528 \pm 20 \mathrm{~nm}$.

Figure 29 - Fluorescence of fluorescein / dabcyl labeled aptamer beacon (FLQ) (10nM) measured over time with equimolar VT1 in TSB media. Inset: closer view of FLQ and FLQ+VT1. Data points are the average of 6 wells over 2 replicates. Error bars represent $95 \%$ Cl. Ex. $495 \pm 10$ nm, Em. $528 \pm 20$ nm.

Figure 30 - Rate of fluorescence change of fluorescein labeled aptamer (FLX), fluorescein / dabcyl labeled aptamer beacon (FLQ), or free fluorescein (FI) at $10 \mathrm{nM}$ in TSB diluted with TES buffer over a 60-minute period. Data points are the average of 6 wells over 2 replicates. Error bars represent 95\% Cl. Ex. $495 \pm 10$ nm, Em. $528 \pm 20$ nm.

Figure 31 - SDS PAGE gel for crude VT1 preparation. Solutions loaded: 1) ladder; 2-6) crude VT2a solutions; 7) crude VT1a solution; 8) commercial VT1a; 9) commercial VT2a; and 10 ) ladder. A and B subunits indicated for CVT1 by red arrows.

Figure 32 - Effect of crude VT1 on fluorescence of A) fluorescein labeled aptamer (FLX) $(10 \mathrm{nM})$ and B) fluorescein / dabcyl labeled aptamer beacon (FLQ) (10 nM) at concentrations of $2.5 \mathrm{nM}$ (VTL) and $6 \mathrm{nM}$ (VTH) of holotoxin over 60 minutes in TES buffer. Data points are the average of 9 samples over 3 replicates. Error bars are SD. Ex. $495 \pm 10 \mathrm{~nm}$, Em. $528 \pm 20 \mathrm{~nm}$.

Figure 33 - Effect of protein on fluorescence of A) fluorescein labeled aptamer (FLX) (10 $\mathrm{nM}$ ) and B) fluorescein / dabcyl labeled aptamer beacon (FLQ) (10 nM) in the presence of VT1, VT2, bovine serum albumin (BSA), or tryptone soya broth (TSB) (10 nM each) in TES buffer. Data points are the average of 3 samples, except for FLQ+VT1 (2 samples). Error bars are SD. Ex. $495 \pm 10 \mathrm{~nm}, \mathrm{Em} .528 \pm 20 \mathrm{~nm}$. 98

Figure 34 - Potential methods by which VT binds additional beacon cooperatively with the $5^{\prime}$ complement region of the aptamer beacon. A) Each VT binds a separate beacon, 
followed by a shift towards toxin complexation. B) Beacon preferentially binds the same toxin, resulting in fewer toxins interacting with a higher number of beacons. 107

Figure 35 - Chemical structures of left) Texas $\operatorname{Red}^{\circledR}-\mathrm{X}$, succinimidyl ester; and right) Cy5 phosphoramidite, 1-[3-(4-monomethoxytrityloxy)propyl]-1'-[3-[(2-cyanoethyl)-(N,Ndiisopropyl phosphoramidityl]propyl]-3,3,3',3'-tetramethylindodicarbocyanine chloride

Figure 36 - Black Hole Quencher 2 CPG, (4'-(4-Nitro-phenyldiazo)-2'-methoxy-5'methoxy-azobenzene-4"-(N-ethyl-2-O-(4,4'-dimethoxytrityl))-N-ethyl-2-O-glycolate-CPG)

Figure 37 - Black Hole Quencher 3 CPG, (3-Diethylamino-5-phenylphenazium-7diazobenzene-4"-(N-ethyl-2-O-(4,4'-dimethoxytrityl))-N-ethyl-2-O-glycolate-CPG) ..... 116

Figure 38 - Chemical structure for NIR-641 N-succinimidyl ester fluorescent dye; 1-Ethyl1-[5-(N-succinimidyloxycarbonyl)pentyl]-3,3,3,3-tetramethyl-indodicarbocyanine chloride.

Figure 39 - Fluorescence of aptamers labeled with fluorescein (FL), Cy5, and Texas Red (TR) in TES buffer. Data points are the average of 9 samples over 3 trials. Error bars indicate SD. FL Ex 495 \pm 10 nm, Em 528 \pm 20 ; TR Ex 596 \pm 15 nm, Em 680 \pm 30 nm; Cy5 Ex $620 \pm 40 \mathrm{~nm}, \mathrm{Em} 680 \pm 30 \mathrm{~nm}$.

Figure 40 - Comparison of the fluorescence of Texas Red / Black Hole Quencher 2 labeled aptamer beacon (TRQ) in the presence of equimolar complete complement DNA (CC) compared to Texas Red labeled aptamer (TRX) and TRQ alone in TES buffer. Data points are the average of 9 wells over 3 replicates. Error bars represent SD. Ex $596 \pm 15$ $\mathrm{nm}, \mathrm{Em} 680 \pm 30 \mathrm{~nm}$

Figure 41 - Comparison of the fluorescence of Cyanine 5 / Black Hole Quencher 3 labeled aptamer beacon (CYQ) in the presence of equimolar complete complement DNA (CC) compared to Cyanine 5 labeled aptamer (CYX) and CYQ alone in TES buffer. Data points are the average of 9 wells over 3 replicates. Error bars represent SD. Ex $620 \pm 40$ $\mathrm{nm}, \mathrm{Em} 680 \pm 30 \mathrm{~nm}$

Figure 42 - Blanked change in fluorescence over time for Texas Red / Black Hole Quencher 2 labeled aptamer beacon (TRQ) (50 nM) in the presence of VT1a, VT2a, or complete complement (CC) each at $50 \mathrm{nM}$ in TES buffer. A) Full view including unquenched Texas Red labeled aptamer (TRX) sequence; B) view excluding complete complement DNA (CC) and TRX curves. Data points are the average of 9 readings over 3 replicates. Error bars show SD. Ex. $596 \pm 15$ nm, Em. $680 \pm 30 \mathrm{~nm}$.

Figure 43 - Blanked change in fluorescence over time for Cyanine 5 / Black Hole Quencher 3 labeled aptamer beacon (CYQ) (50 nM) in the presence of VT1a, VT2a, or 
complete complement (CC) each at $50 \mathrm{nM}$ in TES buffer. A) Full view including unquenched Cyanine 5 labeled aptamer (CYX) sequence; B) view excluding complete complement DNA (CC) and CYX curves. Data points are the average of 9 readings over 3 replicates. Error bars show SD. Ex. $620 \pm 40$ nm, Em. $680 \pm 30 \mathrm{~nm}$.

Figure 44 - Visible fluorescence of fluorescein, Texas Red, and Cy5 solutions from left to right $(100,10,1,0.1$, and $0.01 \mu \mathrm{M}$ from bottom to top) excited with a handheld UV lamp. Image taken with a cell phone camera....

Figure 45 - Comparison of visible fluorescence of fluorescein (left) and Texas Red (right) spotted on untreated TSA plates and allowed to dry. Solutions were 40, 30, 20, 10, 5 , and $1 \mu \mathrm{M}$ going clockwise for each segment and spotted in quintuplicate. Image taken with a cell phone camera.

Figure 46 - Comparison of visible fluorescence of fluorescein and Texas Red spotted on an untreated TSA plate. Solutions were 40, 30, 20, 10, 5, and $1 \mu \mathrm{M}$ from bottom to top for each segment and spotted in triplicate. Image taken with a cell phone camera..... 125

Figure 47 - Comparison of visible fluorescence of fluorescein (left) and Texas Red (right) spotted on untreated $1.5 \%$ agarose plates. Solutions were 40, 30, 20, 10, 5, and $1 \mu \mathrm{M}$ going clockwise for each segment. Samples were spotted in quintuplicate and plates were refrigerated overnight to allow spots to dry. Images taken with a cell phone camera.

Figure 48 - Fluorescence spectra of fluorescein, Texas Red, and Cy5 as well as excitation and emission filters ranges. Absorption spectra are indicated by dashed lines and emission spectra by solid lines. Image made using ThermoFisher's Fluorescence SpectraViewer Web Application.

Figure 49 - Comparison of dichroic and 50/50 mirrors used for fluorescence detection. Left: schematic of the use of a dichroic mirror. Right: schematic of the use of a 50/50 mirror.

Figure 50 - Mechanism for amine-catalyzed $\beta$-elimination causing DNA backbone cleavage at abasic sites.

Figure 51 - Predicted secondary structure of polyA substrate using Mfold software (Zuker, 2003).

Figure 52 - Mean fluorescence values for the enzyme activity assay at time 0 and 40 for both 40 -minute incubations at $30^{\circ} \mathrm{C}$ with polyA background subtracted. Solutions in EAB contained DNase (0.5 units per $100 \mu \mathrm{L})$, VT1 or VT2 (10 nM), polyA (3.75 $\mu \mathrm{M})$, and during the second incubation $\mathrm{N}, \mathrm{N}^{\prime}$-dimethyl-ethylene-diamine $(100 \mathrm{mM})$. Data points the average of 8 samples over 3 replicates. Error bars show original SD. Ex. $495 \pm 10 \mathrm{~nm}$, Em. $528 \pm 20 \mathrm{~nm}$. 139 


\section{List of Appendices}

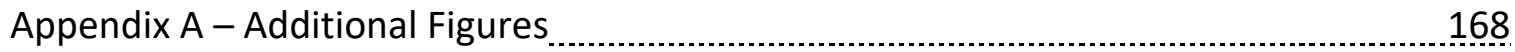

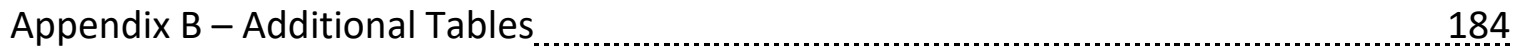

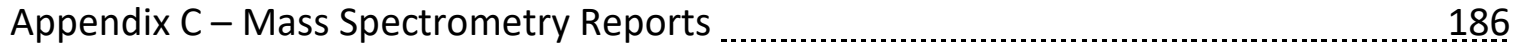




\section{Glossary / List of Abbreviations}

$\alpha$-sarcin - A type I ribosome inactivating protein (RIP) which inactivates ribosomes by cleavage of the phosphodiester bond between G4325 and A4326 in rat 28 S rRNA.

A1 subunit - The enzymatically active portion of the VT A subunit. Amino acids 1-252 in VT1a, 1-251 in VT2a.

A2 subunit - The portion of the VT A subunit which interacts with the B subunit pentamer to form the holotoxin. Amino acids 252-315 in VT1a, 251-319 in VT2a.

A subunit - A $32 \mathrm{kDa}$ peptide subunit which interacts with the $B$ subunit pentamer to form a functional VT holotoxin. Composed of two portions which are cleaved in target cells.

A-4324 - The rRNA location in rat ribosomes that is the catalytic site of action for VT activity. VT cleaves the adenine residue from the RNA sequence causing a cascade of effects, specifically inhibition of protein synthesis and cell death.

$A B_{5}$ toxin - A protein toxin composed of 6 subunits: 1 enzymatically active $A$ subunit, and $5 \mathrm{~B}$ subunits which bind to receptors causing internalization of the toxin.

Abasic sites - Sites within a nucleotide strand which have had the nitrogenous base removed leaving only the phosphate sugar backbone intact.

Affinity - A term which describes the strength of interaction between molecules. The $K_{d}$ is frequently used as a quantitative representation of this affinity for aptamers and their targets.

Antibody - A class of immune particles produced by mammals which contribute to defense against toxins and infectious agents. Generally, refers to Immunoglobulin $\mathrm{G}$ molecules which are also used in biotechnology or detection. Preparation of antibodies can only be done by use of a cell culture and is intrinsically variable from batch to batch as a result.

Antigen - The portion of a molecule to which antibodies can develop affinity.

Aptamer - A class of molecules composed of single-stranded nucleotides which have a high affinity and specificity for target molecules. Aptamers are developed by the SELEX process and are synthesized chemically. Modifications are generally easily incorporated.

Aptamer beacon - An aptamer sequence which has been modified to create a signal upon interaction with the target molecule. This is frequently accomplished using a fluorescent system such as dual-labeling with fluorophore and quencher molecules.

B subunit - A 7.7 kDa peptide sequence which has the property of binding to the Gb3 cell receptor. Upon interaction with $\mathrm{Gb3}$ rich regions of target cells the $\mathrm{B}$ subunits form pentamers, interact with the A subunit, and cause internalization of the VT 
holotoxin. It is 69 amino acids long for VT1, and 71 for VT2.

$\mathrm{CD}_{50}$ - Median cytotoxic dose; the dose required to cause death in $50 \%$ of cells tested.

CFU - Colony forming units; a unit estimating the number of viable bacterial cells in a sample.

$\mathrm{Cl}$-Confidence interval; the range within the true value is expected to lie, given the frequency of this being true statistically.

CPG - Controlled pore glass; the type of columns used for solid support DNA synthesis. The column is silica based with consistent pore size.

DNA - Deoxyribonucleic acid, the genetic information storage molecule. DNA can also be used for nanotechnological purposes including functional molecules, such as aptamers.

Escherichia coli - A species of Gram negative bacteria found in the gastrointestinal tracts of mammals and other animals. Most are commensal in nature, however, a subset of $E$. coli are human pathogens.

eae - The gene encoding intimin, a protein which is required for producing an attaching and effacing phenotype in some pathogenic $E$. coli.

EAEC - Enteroaggregative E. coli, one of seven E. coli pathotypes typified by formation of a thick biofilm of bacteria and production of enterotoxins and cytotoxins.

$\mathrm{EC}_{50}$ - Median effective concentration; the concentration at which the effect is seen in 50 percent of the population tested.

$E D_{50}$ - Median effective dose; the dose at which the effect is seen in 50 percent of the population tested.

EF-1 - Elongation factor 1 found in prokaryotic organisms. It is analogous to the EF-Tu of eukaryotes. EF-1 (and EF-Tu) deliver aminoacyl tRNA as a complex to the ribosome.

EF-2 - Elongation factor 1 found in prokaryotic organisms. It is analogous to the EF-G of eukaryotes. EF-2 (and EF-G) catalyzes the translocation of the peptidyl-tRNA and deacylated tRNA in the ribosome.

EhxA - The gene encoding enterohemolysin. The plasmid which carries this gene has been associated with LEE-positive VTEC and has been used as a virulence marker although the exact role in pathogenicity is not fully known.

ELISA - Enzyme-Linked Immunosorbent Assay, a technique using antibodies to detect or quantify a target molecule through a binding step followed by a linked enzyme's subsequent activity.

Enterobacteriaceae - A taxonomical family of bacteria containing Gram-negative bacteria, including genera Citrobacter, Enterobacter, Salmonella, Escherichia, Yersinia pestis, Klebsiella, Shigella, among many others.

ER - Endoplasmic reticulum, one of the organelles of the cell. The ER is involved in 
intracellular trafficking and preparing peptides for secretion or membranes.

FAM - Fluorescein amidite; fluorescein modified with protecting groups and

functionalized to be added to a synthetic nucleotide sequence.

FITC - Fluorescein isothiocyanate; fluorescein modified with isothiocyanate functional group to add amine-reactivity. More recently succinimidyl-ester modified fluorescein is used instead.

Fluorescein - A commonly used yellow-green fluorophore with excitation peaking at about $494 \mathrm{~nm}$ and emission peaking at about $512 \mathrm{~nm}$. Can be functionalized for labeling proteins and DNA (FAM / FITC).

FRET - Fluorescence resonance energy transfer; transfer of light energy from a donor molecule to an acceptor $r$. The energy from the first molecule is transferred to excite the second. This is frequently used as a means of detection for a specific interaction. Only once the interaction has brought the two FRET molecules together will the signal from the second one be visible.

fur locus - The genetic location of the Fur protein.

Fur protein - A protein acts to regulate expression of genes related to iron-binding and uptake. When there are high-levels of iron the genes affected by the Fur protein regulation are repressed. The VT1 promoter region shares homology with the genes repressed by Fur protein.

Furin - The main enzyme involved in splitting the A subunit into A1 and A2 for activity in the cell by activity on the cleavable loop portion.

Gb3 - Globotriose receptor, composed of the trisaccharide (Gal $\alpha 1-4 \mathrm{Gal} \beta 1-4 \mathrm{Clc}$ ) attached to the lipid, ceramide. Gb3 is found more commonly in lipid rafts of the cell membrane, but only on certain cell types. Also known as glycolipid receptor CD77. The saccharide portion is also known as Pk trisaccharide.

Gram-negative - A designation given to bacteria with only a thin cell wall (composed of peptidoglycan) between an outer membrane and an inner plasma membrane. When stained according to Gram's method, Gram-negative bacteria lose the crystal violet stain (and take the color of the red counterstain). Gram-positive bacteria have only one cellular membrane within a thick cell wall, resulting in retention of the crystal violet stain. E. coli are Gram-negative bacteria.

GTP - Guanosine triphosphate. An important nucleotide monomer for RNA used as a source of energy in a number of enzymatic reactions as well as signal transduction. GTPase - GTP-binding proteins, or G-proteins, catalyze the hydrolysis of GTP. They are an important component of signal transduction.

$\mathrm{H}$-type - The classification of $E$. coli based on serological reaction of flagellar protein to a standard set of antibodies. There are 53 different accepted $\mathrm{H}$-types.

$\mathrm{HC}$ - Hemorrhagic colitis, a disease syndrome resulting in bloody diarrhea. 
HRMEC - Human renal glomerular microvascular endothelial cells.

HSVEC - Human saphenous vein endothelial cells.

HUS - Hemolytic uremic syndrome, a severe disease process resulting from VTEC infection. HUS is described as a combination of microangiopathic hemolytic anemia (death of red blood cells, especially in small blood vessels), thrombocytopenia (low levels of platelets), and acute nephropathy (kidney disease / damage). For details refer to Section 1.2.5.

HUVEC - Human umbilical vein endothelial cells.

$\mathrm{IC}_{50}$ - Median inhibitory concentration; the concentration required to cause inhibition in 50 percent of the population tested.

$I D_{50}-$ Median inhibitory dose; the dose required to cause inhibition in 50 percent of the population tested.

IL - Interleukin, a family of pro-inflammatory cytokines implicated in sensitizing patients to development of HUS in VTEC infections.

$K$ type - A serological classification for $E$. coli based on reaction of capsular polysaccharides with a standard set of $\mathrm{K}$ antibodies.

$\mathrm{K}_{\mathrm{d}}$ - Dissociation constant, a quantitative measurement of affinity between ligand and target, such as aptamers and their targets.

Kinase - A class of enzymes involved in phosphorylation of other proteins.

Late genes - Phage genes encoding production of phage particles and other related systems. They are only significantly upregulated when the phage is induced into the lytic cycle. VT genes are encoded in this part of the phage genome.

$\mathrm{LD}_{50}$ - Median lethal dose; the dose at which $50 \%$ of individuals treated result in death. LPS - Lipopolysaccharide, also known as endotoxin. Lipopolysaccharides are expressed on the outer membrane surface of $E$. coli. It is composed of an outer O-antigen (by which O-types are distinguished) attached by other saccharides to a lipid base which is embedded in the membrane.

Lysogenic cycle - Or lysogeny, is one of two cycles of viral reproduction. The lysogenic cycle is characterized by the integration of viral genes in the host genome, where it is replicated to daughter cells through host cell replication. The viral genes will remain integrated within the host genome until the lytic cycle is induced.

Lytic cycle - Or lysis, is one of two cycles of viral reproduction. The lytic cycle is characterized by excision of viral genes from the host genome, replication of viral particles, and resulting lysis of the host cell releasing viral particles into the environment. The released viruses can then infect other cells.

Macropinocytosis - A general non-selective process for the uptake of molecules by single-celled organisms.

MPN - Most probable number; a method used to approximate the number of bacterial 
cells present. The method involves diluting a solution multiple times and by using replicated determining the presence or absence at those lower concentrations.

mRNA - Messenger RNA, is transcribed from the genetic information on DNA and takes

it for translation into proteins outside the nucleus.

$\mathrm{NM}$ - Non-motile, the H-type designation given to $E$. coli not expressing flagellin. non-0157 - A broad category of VTEC which do not have the 0157 serotype.

$O$ rough - The O-type distinction given to E. coli not expressing or only partially expressing LPS resulting in no reactivity to typing antibodies.

O type - A classification for $E$. coli based on reaction of LPS polysaccharide with a standard set of $\mathrm{O}$ antibodies. There are currently 185 accepted $\mathrm{O}$-types.

0157 - The O-type historically most commonly associated with VTEC outbreaks and illnesses in North America, especially O157:H7 and 0157:NM strains. 0157 strains are typically highly pathogenic with VT2- and LEE-positive genotypes. 0157 strains generally can be distinguished by lack of sorbitol fermentation and $\beta$ -

glucuronidase activity, although isolates can be found without both of these traits.

Operon - A single functioning section of DNA with a promoter region, regulating operator region, and associated genes.

Outbreak - A series of illnesses caused by a single strain or very closely related strains over a short period of time by the same vector.

Pathotype - Groups of pathogenic E. coli that cause enteric illness based on virulence factor expression and the mechanisms by which they induce disease. There are currently seven accepted pathotypes for E. coli: enteropathogenic E. coli (EPEC); enteroinvasive $E$. coli (EIEC); enteroaggregative $E$. coli (EAEC); enterotoxigenic $E$. coli (ETEC); diffusely adherent E. coli (DAEC); adherent invasive E. coli (AIEC); and verotoxin-producing $E$. coli (VTEC).

PCR - Polymerase chain reaction; a method of amplifying specific portions of a DNA sample by using DNA primers, a polymerase enzyme, and nucleic monophosphates in solution. The solution is heated and cooled in cycles causing the DNA sequence of choice to be copied over and over if it is in the sample. This can be used for detection of specific $E$. coli genes in screening.

Periplasmic space - The space between the inner cytoplasmic membrane and the bacterial outer membrane in Gram-negative bacteria.

PFGE - Pulsed field gel electrophoresis, a method that results in unique patterns based on partial digestion of whole genome. Restriction enzymes cut as specific sequences throughout the genome. These DNA fragments are then able to be separated by electrophoresis. These patterns can be compared to other isolates to determine a measure of relatedness or identify outbreaks.

Phage titer - A measure of the concentration of phage particles in a liquid culture 
determined by diluting the original solution and observing the resulting lytic effect on susceptible bacteria.

Pk trisaccharide - Another name for the saccharide portion of Gb3 (Gal $\alpha 1-4 \mathrm{Gal} \beta 1-4 \mathrm{Clc}$ ), more commonly used in studies of blood.

Quorum sensing - A system of stimuli and response correlated to bacterial population density which results in upregulating expression of certain genes.

Restriction enzymes - Enzymes that cleave DNA at specific sequence recognition sites.

Retrograde transport - The name for the transport system which can transport

molecules from the exterior of the cell via endosomes to the trans-Golgi network, through the Golgi apparatus, to the endoplasmic reticulum (ER).

Ricin - A type II ribosome inactivating protein highly homologous to VT produced by the castor-oil-plant, Ricinus communis.

RIPs - Ribosome inactivating proteins, a class of proteins that inactivate protein synthesis by irreversible modification of the ribosome RNA.

RNA - Ribonucleic acid, nucleotides actively involved in cellular process of protein translation. The main forms it takes are as: messenger RNA (mRNA); transfer RNA (tRNA); and ribosomal RNA (rRNA) although other RNAs also exist in the cell.

RPTEC - Renal proximal tubule epithelial cells.

rRNA - Ribosomal RNA, the nucleic acid component of ribosomes.

SD - Standard deviation; a measure of the variance within a set of samples.

SELEX - Selective Evolution of Ligands by Exponential Enrichment, the name for the cyclical method by which aptamers are selected. A randomized pool of nucleotides is selected for strong, selective binding to a target molecule by cycles of binding, partitioning, and amplification.

Serotype - The resulting numerical designation to distinguish $E$. coli strains based on antibody reactions to cell surface antigens $(\mathrm{O}, \mathrm{K}$, and $\mathrm{H})$. The reactions result in a numerical O:K:H designation scheme, however generally only the O:H serotype, for example 0157:H7, is used to type isolates.

SOS response - A pathway involved in response to general DNA damage. Activation of the SOS response causes induction of VT phages, and associated VT production.

Sporadic illness - Illness caused as an isolated case, not resulting in multiple illnesses. SPR - Surface plasmon resonance, a method of detecting interactions between molecules by adhering one to a metallic surface and measuring the change in resonant angle. All angles will reflect except the resonant angle which will continue parallel to the surface. As binding occurs the resonant angle changes and can be observed.

SRL - The sarcin-ricin loop, a highly conserved region of rRNA in the large ribosomal subunit which plays a substantial role in binding elongation factors. The SRL is the 
target for VT activity as well as other RIPs.

Strain - A genetically homologous population of bacteria resulting from clonal descent from a single common ancestor.

Subtype - VT variants can be grouped based on gene and peptide sequence homology.

There are 3 VT1 subtypes (a, c, d) and 7 VT2 subtypes (a, b, c, d, e, f, g).

tRNA - Transfer RNA, small single stranded RNA which binds amino acids and transports them to the ribosome for peptide elongation.

UDG - Uracil-DNA glycosylase, an enzyme which prevents uracil monomers from addition into DNA by cleaving the $\mathrm{N}$-glycosylic bond, promoting other enzymes to initiate repair pathways without removing the whole base by its own activity.

Untypable - The designation given to $E$. coli strains that do not possess standard $O$ and $\mathrm{H}$ serotypes.

Variant - A VT that, while highly related to the subtype toxin differs by one or more amino acid. Variants are still classified by subtype, but are additionally given their own unique designation.

VBNC - Viable but nonculturable cells, a state which some bacteria can enter during stress conditions. This is a metabolic state in which the cells may persist for prolonged periods without replication. They are generally nonculturable by standard techniques. VBNC VTEC have been found to produce VT.

Vero cells - A cell line derived from green monkey kidneys that is susceptible to verotoxin cytotoxicity.

Virulence factor - A trait that contributes to the pathogenic processes of a bacteria. Bacteria can possess multiple virulence factors.

VT (also known as SLT or Stx) - Verotoxin (verocytotoxin, Shiga-like toxin, or Shiga toxin), the definitive virulence factor expressed by VTEC. A protein toxin which acts as a type II RIP with a catalytic A subunit and a B subunit pentamer. Discussed in detail in section 1.1.

VT1 - The first of two types of VT with three subtypes: 1a, 1c, and 1d. VT1 is antigenically identical to the Shiga toxin produced by Shigella dysenteriae with as few as one amino acid differences for VT1a.

VT2 - The second of two types of VT with seven subtypes: $2 a, 2 b, 2 c, 2 d, 2 e, 2 f$, and $2 g$. VT2a is antigenically distinct from Shiga toxin produced by Shigella dysenteriae sharing only about $56 \%$ amino acid identity in 2 a.

VTEC (also STEC or SLTEC) - Verotoxin-producing Escherichia coli (Shiga toxin-producing E. coli or Shiga-like Toxin-producing E. coli), one of seven E. coli pathotypes typified by expression of VT.

$v t x$ genes (also known as stx) - Genes encoding verotoxin encoded in the late gene section of a bacteriophage. 


\subsection{Introduction}

\subsection{Verotoxin (Shiga Toxin, Shiga-like Toxin; VT)}

\subsubsection{General Information and Structure}

Verotoxin (VT) was originally discovered as a toxin with an irreversible cytotoxic effect on Vero cells (a cell line derived from African green monkey kidney epithelium) (Konowalchuk et al., 1977). This toxin was produced by a subset of Escherichia coli (E. coli) strains and the toxicity was unrelated to other exotoxins. They were named verotoxins (VTs) due to this toxicity in Vero cells (Konowalchuk et al., 1977). VTs are also known as Shiga toxins (Stxs) or Shiga-like toxins (SLTs) with the E. coli producing VT known as verotoxin-producing E. coli (VTEC), Shiga toxin producing E. coli (STEC), or Shiga-like toxin producing E. coli (SLTEC). This is due to VT sharing very high homology and antigenic identity with the toxin produced by Shigella dysenteriae, Shiga toxin.

E. coli producing VT were first implicated in outbreaks of disease in the 1980s (Riley et al., 1984; Karmali et al., 1983). Some patients were observed to develop serious disease outcomes such as hemorrhagic colitis (HC) and hemolytic uremic syndrome (HUS). It quickly became evident that Shiga-like toxin and verotoxin being studied by different groups were variants of the same toxin (Strockbine et al., 1986) and it was later proposed that the nomenclature for the toxins, and E. coli that produce them, be used interchangeably. However, different geographical regions still use one set of terms or the other (VT/VTEC or Stx/STEC). For this report, the terms VT and VTEC will be used. At the time of discovery, the toxins were observed to divide into at least two 
antigenic groups (Konowalchuk et al., 1977). VT was confirmed to fall into two antigenic types, VT1 and VT2, based on their similarity to Shiga toxin (Strockbine et al., 1986). VT1 toxins are the most similar to Shiga toxin with only a single amino acid difference in the prototype VT1a and the ability to cross-react with antibodies for the Shiga toxin. VT2 toxins share less amino acid identity with VT1 (VT2a sharing 56\%) and are antigenically distinct (Scheutz et al., 2012; Jackson et al., 1987). VT1 and VT2 can be further differentiated into three VT1 (1a, 1c, 1d) and seven VT2 (2a, 2b, 2c, 2d, 2e, 2f, 2g) subtypes based on gene sequence (Scheutz et al., 2012). Within VT1 or VT2 the subtypes have $90 \%$ or greater amino acid identity, aside from VT2f which is about $60 \%$ identical to VT2a (Schmidt et al., 2000).

Verotoxin (VT) is an $A_{5}$ protein with a single $A$ subunit ( $32 \mathrm{kDa}$ ) that is noncovalently associated with a pentamer of B subunits (7.7 kDa each) (Melton-Celsa, 2014; Fraser et al., 1994). Unlike other $A B_{5}$ toxins VT is not secreted as holotoxin, but is released as individual subunits through lytic activity of the VT bacteriophage (Neely and Friedman, 1998). Combination of subunits is facilitated by B subunit interaction with Gb3 receptors (Pellino et al., 2016), with the A subunit subsequently binding to form holotoxin (Figure 1). The B subunits bind to $\mathrm{Gb} 3$ cell receptors and cause internalization of the holotoxin. The A subunit is composed of two portions, A1 and A2, held together by a disulfide bridge and a short loop of nucleotides (Fraser et al., 1994). Inside the cell the $A$ subunit is transported, cleaved, releasing the enzymatically active $A 1$ subunit, and halts protein synthesis by irreversible modification of the $28 \mathrm{~S}$ ribosomal RNA (rRNA), leading to cell death. This will be described in more detail below. 
A

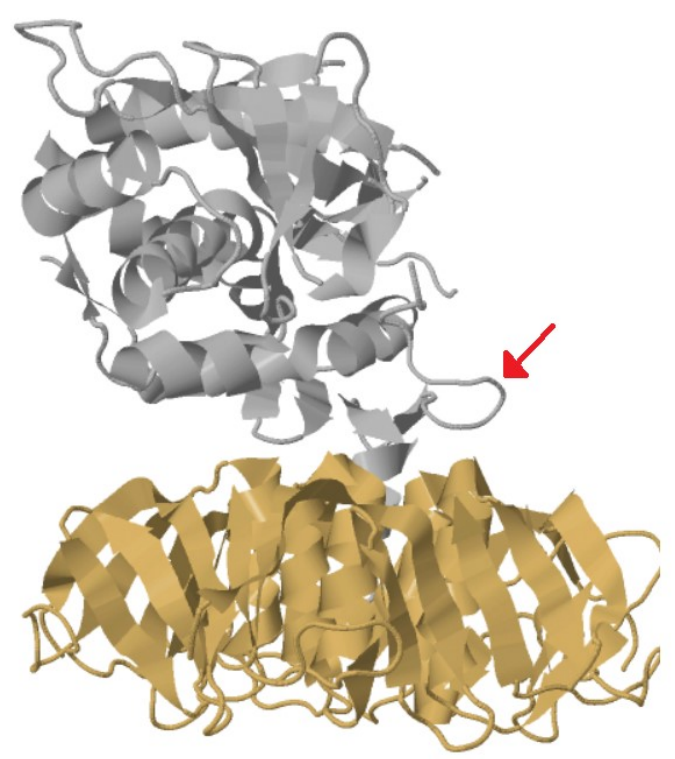

B

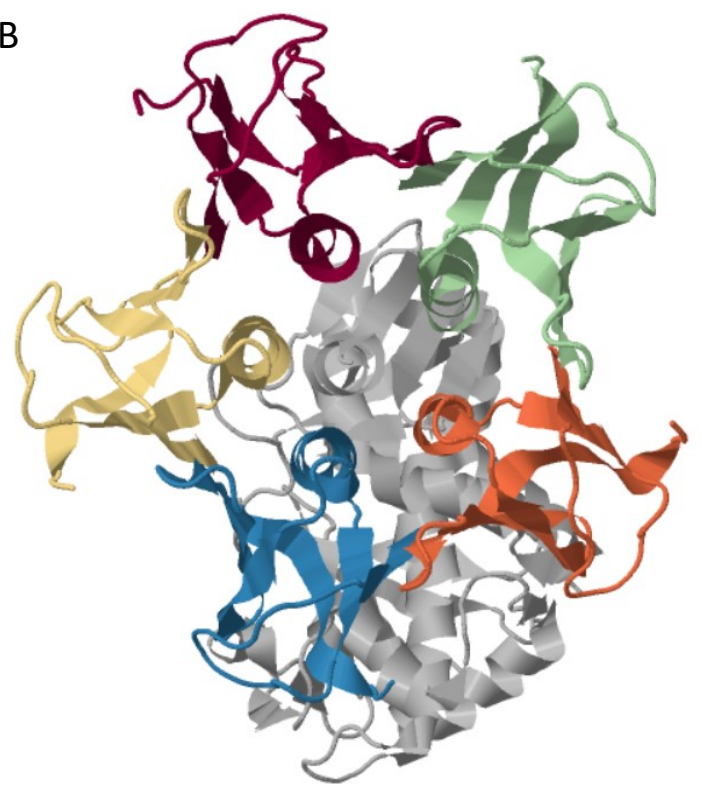

Figure 1 - Verotoxin holotoxin structure 3D model. A) A subunit (silver) interacting noncovalently with the $B$ subunit pentamer (gold) via the $A 2$ chain. A red arrow indicates the cleavable loop. B) The five B subunits coloured differently. Model produced by JSmol web application from Protein Data Bank entry 1DMO: SHIGA TOXIN (Fraser et al., 1994).

\subsubsection{Enzymatic Activity and Target}

Enzymatically, VT is part of the ribosome-inactivating protein (RIP) family of protein toxins (Reyes et al., 2012). The active site of VT is highly conserved among RIPs and is analogous to that of ricin (Fraser et al., 1994). Due to VTs multimeric structure, it is classified as a type II RIP. RIPs have activity targeting a highly conserved stem loop in the $28 \mathrm{~S}$ rRNA called the sarcin-ricin loop (SRL) after the RIPs a-sarcin and ricin which were first found to target it and were used extensively in its study (Chan et al., 1983). The A1 subunit of VT specifically has catalytic $\mathrm{N}$-glycosidase activity and cleaves the adenine at $\mathrm{A}-4324$ (numbering for rat ribosomes) causing protein synthesis to stop by preventing peptide elongation (Figure 2) (Endo et al., 1988b; Reisbig et al., 1981). Ribosomes are found to survive nuclease treatment resulting in multiple nicks, but a 
single modification to the SRL can result in complete inactivation. As such the SRL is a crucially important sequence (Chan et al. 1983).

The SRL is the longest universally conserved ribosomal sequence across species (Gorski et al., 1987). The SRL forms a hairpin (or stem-loop) structure ending with a GAGA tetraloop. The hairpin starts with a standard base-paired helix followed by a flexible non-Watson-Crick region, a G-bulged cross-strand A stack and finally the tetraloop (Correll et al., 1998; Szewczak and Moore, 1995). The SRL region is important for peptide elongation (Reisbig et al., 1981) and has been implicated in tRNA binding (Igarashi et al., 1987), elongation factor 1 (EF-1) and elongation factor 2 (EF-2) dependent GTPase hydrolysis and translocation (Shi et al., 2012; Clementi et al., 2010; Moazed et al., 1988), as well as adding stability to the translocation complexes during translation (Shi et al., 2012). As such, cleavage by VT stops those interactions preventing catalytic protein synthesis.
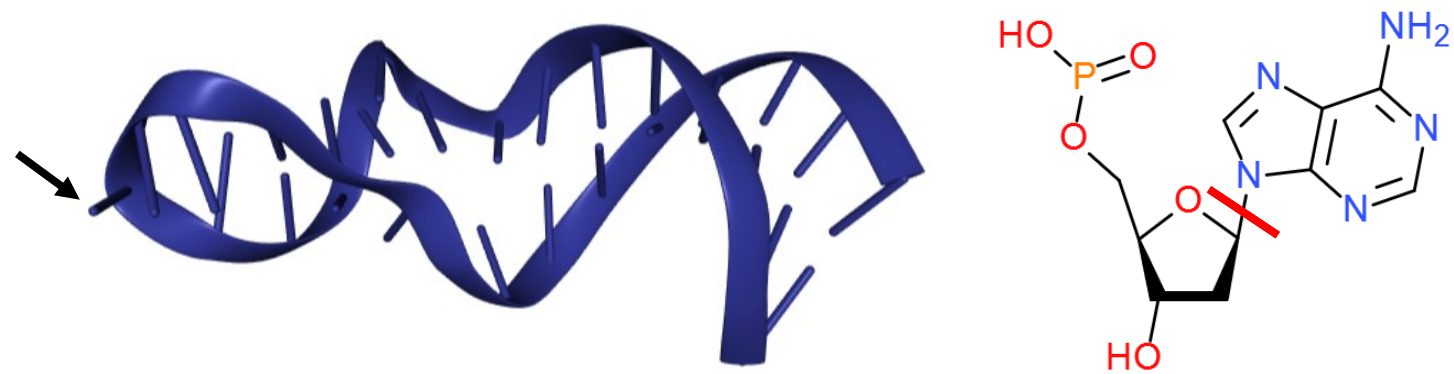

Figure 2 - The structure of the SRL region targeted by verotoxin. Left) the stem loop structure of $S R L$ portion of the large ribosomal rRNA subunit; Adenine-4324 indicated by an arrow. Right) the structure of deoxyadenosine monophosphate with the cleavage site indicated by thick red line. SRL model produced by NGL web application from Protein Data Bank entry 430D (Correll et al., 1998); Chemical structure produced with ChemDoodle web application.

In addition to cleavage of the SRL loop in RNA it was discovered that all RIPS tested (53 RIPS including VT1) cleave multiple adenine residues from RNA and DNA substrates. As such, it was proposed that the RIP family be renamed polynucleotide: 
adenosine nucleosidases (Barbieri et al., 1994; 1997; 1998). Isolated VT1 A1 subunits have been reported to cleave DNA (Barbieri et al., 1998). In human tissue cultures treated with 0.01 nM VT1, nuclear DNA damage was observed (Brigotti et al., 2002). Human umbilical vein endothelial cells (HUVEC) treated with 0.01 nM VT1 were observed to have DNA damage that was induced well before typical apoptotic events strongly suggesting that the damage was caused by the toxin activity rather than from apoptotic response induction as a result of protein synthesis inhibition (Brigotti et al., 2002). Additionally, inducing protein synthesis inhibition with cycloheximide did not result in DNA damage or apoptosis indicating that inhibition of protein synthesis is not enough to cause apoptosis (Brigotti et al., 2002). Together these studies show that VT in addition to cleaving the SRL also cleaves RNA and DNA (Brigotti et al., 2001).

No cofactors have been identified that enhance the enzymatic activity of VT. Potential cofactors tested include: $\mathrm{Co}^{2+}, \mathrm{Mn}^{2+}, \mathrm{Mg}^{2+}$, and $\mathrm{Ca}^{2+}$ (Brigotti et al., 2001); NAD, ATP, NADP, NADH, and pH 5 supernatant (with elongation factors and aminoacyltransferases) (Reisbig et al., 1981); or ATP, post ribosomal supernatant, and a preparation of tRNAs (Brigotti et al., 1997). In general DNA glycosylases do not require metal ions as cofactors or a source of energy (Dodson et al., 1994).

Cleavage of the A1 subunit from VT is required for activity since a portion of the A2 chain blocks the active site (Fraser et al., 1994; Fraser et al., 2004). Studies on the kinetic effects of cleaving the VT1 holotoxin indicate that interaction and binding to the ribosome is not affected by the state of the toxin, while the catalytic activity is greatly enhanced (1000X) by activating treatment (Brigotti et al., 1997). In vitro activation has 
historically been performed through treatment with trypsin, dithiothreitol (DTT), and either urea or sodium dodecyl sulfate (SDS) (Reisbig et al., 1981; Brigotti et al., 2001).

\subsubsection{Mode of Action In Vivo}

1.1.3.1 B Subunit: Receptor Binding, Holotoxin Formation, and Uptake Following release of toxin subunits, the first steps for the toxin are largely mediated by the interaction of the B subunits with cellular receptors. The main receptor for B subunits is globotriaosylceramide, Gb3 (Jacewicz et al., 1986). Gb3 is composed of a trisaccharide (Gal 1 1-4Galß1-4GIc), also called Pk trisaccharide, which is attached to ceramide. These glycolipids are generally concentrated in lipid rafts in cell membranes (Kovbasnjuk et al., 2001). In humans, Gb3 receptors are located in: red blood cells of the Pk group (Newburg et al., 1993); kidney endothelial and epithelial cells; microvascular epithelial cells in the intestine; platelets; subsets of germinal centre B lymphocytes; endothelial and dorsal root ganglion cells in the peripheral nervous system; on endothelial cells and neurons in the central nervous system (CNS) (Obata et al., 2008; Ren et al., 1999); and overexpressed in a number of cancer tissues (Johannes and Römer, 2010).

The B subunits bind to Gb3 with multiple binding sites per B monomer in a sequential cooperative model (Yung et al., 2003). Binding of the B monomers increased local concentration and enhances B pentamer formation (Pellino et al., 2016). The binding strength of the pentamer has been shown to have a greatly increased binding affinity $\left(4.7 \times 10^{9} \mathrm{M}^{-1}\right)$ compared to monomers $\left(\sim 10^{3} \mathrm{M}^{-1}\right)$ (St. Hilaire et al., 1994; Fuchs 
et al., 1986). This association allows the subunits to combine and further stabilize the holotoxin, with evidence suggesting that the $A$ subunit has a role in enhancing this association (Pellino et al., 2016).

Internalization and transport was reviewed in Bergan et al., 2012, but will be outlined here briefly. Toxin binding to Gb3 receptors causes conformational changes to the cellular membrane resulting in transportation into the cell (Römer et al., 2007). Additionally, macropinocytosis (a general non-selective uptake method) can result in toxin internalization (Malyukova et al., 2009). Aside from transport into the cell, VT also interacts with neutrophils by the TLR4 receptor, which can act to transport the toxin through the blood to target organs with high Gb3 expression (Brigotti et al., 2013).

\subsubsection{A Subunit: Retrograde Transport and Ribosome Inactivation}

Once the toxin has been transported into the cell the A subunit must be cleaved, reduced, and delivered to the cytosol before interaction with the ribosomes can occur. Transport of the A subunit occurs via endosomes to the trans-Golgi network, through the Golgi apparatus, and to the endoplasmic reticulum (ER) in a complex pathway known as the retrograde transport pathway (Tesh, 2011; Tam and Lingwood, 2007). Early in this process the cleavable loop is nicked by the endoprotease, furin (Garrad et al., 1995). The disulphide bridge is reduced later in the ER lumen separating the A1 subunit. Use of the ER-associated protein degradation pathway for misfolded proteins has been indicated in transporting A1 into the cytosol (Yu and Haslam, 2005) with evidence that the cleavable loop portion is recognized by those chaperone proteins 
(LaPointe et al., 2005). After translocation only about $4 \%$ of undegraded A1 makes it to the cytosol, an estimated one A1 subunit per cell at the median cytotoxic dose $\left(C D_{50}\right)$ (Tam and Lingwood, 2007).

In the cytosol, A1 locates ribosomes and the SRL adenine. Interaction with the ribosomal stalk proteins has been proposed to play a role in the high enzymatic activity and specificity of RIPs (Tumer and Li, 2012). The ribosomal stalk in both prokaryotes and eukaryotes is a flexible protein structure extending from the large ribosomal subunit that has a role in recruiting translation factors and by doing so enhancing the process of protein synthesis (Gonzalo and Reboud, 2003). The SRL and ribosomal stalk work together as the main interaction site for elongation factors. Multiple lines of evidence (reviewed in Tumer and Li, 2012) point to interaction with the ribosomal stalk as a means of localization to the SRL (Tumer and Li, 2012). This explains the often-observed phenomenon that RIPs cleave intact ribosomes with greater affinity than individual ribosome subunits (Endo and Tsurugi, 1988).

Once localized to the SRL, the A1 subunit cleaves the adenine at position 4324 of eukaryotic 28S rRNA preventing tRNA binding and consequently protein synthesis by the ribosome (Saxena et al., 1989). VT has also been found in nuclear fractions where it can cleave adenine from chromosomal DNA (Suzuki et al., 2000). In addition to those activities further described in Section 1.1.2, VT has been shown to cause apoptosis via both extrinsic and intrinsic signalling pathways through the ribotoxic stress response, the unfolded protein response, and activation of kinase cascades. Although the exact pathways activated vary depending on the cell type (reviewed in Tesh, 2011). 


\subsubsection{Systemic Illness}

VT produced in the intestines interacts with endothelial cells and leukocytes. This causes release of various signalling factors and progression of damage to the intestinal lining; causing bleeding which may enhance VTEC survival (Johannes and Römer, 2010; Law and Kelly, 1995) and making it easier for toxin to enter the circulatory system (Exeni et al., 2007). Toxin also passes into the bloodstream as an effect of translocation by leukocytes (Hurley et al., 2001), or by transcellular trafficking by epithelial cells (Acheson et al., 1996). The toxin is then carried by white blood cells to kidneys and other susceptible tissues where further damage is done by the same mechanism (te Loo et al., 2001; te Loo et al., 2000). White blood cells can also transport lipopolysaccharide (LPS) (Takeshita et al., 1999). Interaction of the toxin and LPS with host immune responses causes a complex system of chemical regulation that may enhance Gb3 concentrations on certain cells, cause further inflammatory response, and promote thrombus formation (Karpman and Ståhl, 2014). The presence of the cytokine TNF- $\alpha$ and VT caused a decrease in thrombomodulin protein expression in glomerular endothelial cells. Thrombomodulin works with $\alpha$-thrombin to degrade clotting factors. The reduction in thrombomodulin enhances thrombi formation (Fernández et al., 2003). Additionally, platelets and Pk group red blood cells are lysed by interaction with the toxin. Blood cell debris, thrombi formation, and apoptosis of renal cells leads to kidney damage and potential kidney failure (Karpman and Ståhl, 2014). VT has been found to alter the permeability of the blood brain barrier (D'Alessio et al., 2016) and in the brain, can cause apoptosis and inflammatory response by interaction with neurons and endothelial 
cells causing CNS damage and other neurological problems (Karpman and Ståhl, 2014).

\subsubsection{Toxicity of Verotoxin}

Toxicity of VT at the cellular level is related primarily to the ability to stop protein synthesis, cleave nuclear DNA, and by doing so cause cellular apoptosis. While toxicity at the organism level is related to inducing systemic illness (HUS, neurologic symptoms, etc.) by interaction with target organs. However, as described in Section 1.1.3.3, the ability of the toxin to cause systemic damage is largely mediated by complex host responses. Since there is no cure for serious complications, voluntary infection studies are not possible, leaving only animal models and case investigations as sources of information.

There is currently no animal model that mimics all aspects of VT-induced illness in humans, although different models offer information on different aspects of illness (colonization, kidney damage, lethal doses, etc.) (Pacheco and Sperandio, 2012; Andreoli et al., 2002). Mice require very high doses ( $10^{5}$ colony forming units (CFU) or higher) for VTEC colonization to occur, but do not develop gastrointestinal symptoms, although mild kidney damage distinct from that in human illness is observed (Pacheco and Sperandio, 2012; Tesh et al., 1993). Gnotobiotic piglets develop many more symptoms from colonization lesions and kidney damage, but do not develop HUS and require complex facilities for testing (Pacheco and Sperandio, 2012). Similarly, infant rabbits allow for observation of colonization, lesion formation, and intestinal symptoms, but not HUS since rabbits do not produce Gb3 receptors in their kidney cells (Pacheco and 
Table 1 - Summary of select VT or Shiga Toxin toxicity trials

\begin{tabular}{|c|c|c|}
\hline Model* & Toxicity (Toxin) and other information" & Reference \\
\hline Baboons & $\begin{array}{l}\text { i.v. } 25 \mathrm{ng} / \mathrm{kg} \text { show symptoms (VT2), no symptoms with } \\
\text { VT1 or control }\end{array}$ & $\begin{array}{l}\text { Siegler et al., } \\
2003\end{array}$ \\
\hline Baboons & $\begin{array}{l}\text { i.v. 10, 50, } 100 \mathrm{ng} / \mathrm{kg} \text { resulted in 0, 80, and 100\% death } \\
\text { (VT1), 25, 100, 100\% (VT2) death. VT2 died slower, but } \\
\text { with lower concentrations. } \\
\text { Dose-dependent disease progression, different cytokine } \\
\text { and biomolecule levels observed }\end{array}$ & $\begin{array}{l}\text { Stearns- } \\
\text { Kurosawa et al., } \\
2010\end{array}$ \\
\hline $\begin{array}{l}\text { Rabbits (New } \\
\text { Zealand white) }\end{array}$ & $\begin{array}{l}\mathrm{LD}_{50}=0.2 \mu \mathrm{g} / \mathrm{kg}(\mathrm{VT} 1), \mathrm{Gl} \text { and CNS damage, no renal } \\
\text { damage. Blood serum } \mathrm{t}_{1 / 2} \approx 2 \text { minutes. Found in } \mathrm{Gl} \text {, brain, } \\
\text { and spinal cord in naïve rabbits; primarily in liver, lung, } \\
\text { spleen, and kidney in immunized. }\end{array}$ & $\begin{array}{l}\text { Richardson et } \\
\text { al., } 1992\end{array}$ \\
\hline $\begin{array}{l}\text { Rabbits; } \\
\text { Mice (albino } \\
18-22 \mathrm{~g} \text { ) }\end{array}$ & $\begin{array}{l}\text { Neurotoxic effects in mice and rabbits; } \\
\mathrm{LD}_{50}=140 \mu \mathrm{g} \text { (Shiga toxin) with i.v. } \mathrm{LD}_{50} \text { much lower } \\
\text { (uncalculated) with more than half at } 59.5 \mu \mathrm{g} \text { dying. } \\
\text { Intoxication irreversible within } 0.5 \text { hours in some. }\end{array}$ & Howard, 1955 \\
\hline Mice & $\begin{array}{l}\text { Oral doses resulted in death (only VT2) and only at higher } \\
\text { expression levels. Died of acute renal tubular necrosis, } \\
\text { while glomerular cells were unaffected. }\end{array}$ & $\begin{array}{l}\text { Wadolkowski et } \\
\text { al., } 1990\end{array}$ \\
\hline $\begin{array}{l}\text { Mice (BALB/c } \\
12 \text { - to } 16-w . o .)\end{array}$ & $\begin{array}{l}\text { Found in nasal turbinates, lungs, and kidneys. 10x more } \\
\text { VT1 located at the lungs, while VT2 targeted the kidneys } \\
2.7 x \text { more. Blood serum } t_{1 / 2}=2.7 \text { (VT1), } t_{1 / 2}=3.9 \text { (VT2) }\end{array}$ & $\begin{array}{l}\text { Rutjes et al., } \\
2002\end{array}$ \\
\hline $\begin{array}{l}\text { Mice (seven } \\
\text { strains) }\end{array}$ & $\begin{array}{l}\mathrm{LD}_{50}=350-900 \mathrm{pg}(\mathrm{VT} 2) \text {; allelic decrease in macrophage } \\
\text { production of TNF and IL-1 resulted in greater protection }\end{array}$ & $\begin{array}{l}\text { Barrett et al., } \\
1990\end{array}$ \\
\hline $\begin{array}{l}\text { Mice (BALB/c } \\
\text { ९, 5-6 w.o.) }\end{array}$ & $\begin{array}{l}\text { Oral } \mathrm{LD}_{50}=2.9 \mu \mathrm{g}(\mathrm{VT} 2) \text {, no death up to } 157 \mu \mathrm{g}(\mathrm{VT} 1) ; \text { no } \\
\text { intestinal lesions, but distal renal tubular damage was } \\
\text { observed. }\end{array}$ & $\begin{array}{l}\text { Russo et al., } \\
2014\end{array}$ \\
\hline $\begin{array}{l}\text { Mice (BALB/C } \\
\text { \$) }\end{array}$ & $\begin{array}{l}\text { Oral doses. Found in kidneys, less in liver and spleen. } \\
\text { Concurrent dosage (VT2 and VT1) extended time before } \\
\text { death and slowed symptom development. Pre-dosage } \\
\text { (VT1) furthered the effect. VT1 toxoid was equivalent. }\end{array}$ & $\begin{array}{l}\text { Russo et al., } \\
2016\end{array}$ \\
\hline $\begin{array}{l}\text { Mice (BALB/C } \\
\& \mathrm{C} 57 \mathrm{BL} / 6 \text { ơ) }\end{array}$ & $\begin{array}{l}\text { Decreased neutrophils caused reduced susceptibility to } \\
\text { VT2 }\end{array}$ & $\begin{array}{l}\text { Fernandez et } \\
\text { al., } 2006\end{array}$ \\
\hline $\begin{array}{l}\text { Mice }(\text { CD-1 o'); } \\
\text { Vero cells; } \\
\text { RPTEC }\end{array}$ & $\begin{array}{l}\text { i.p. } \mathrm{ED}_{50}=2.4 \mathrm{ng}(\mathrm{VT} 2 \mathrm{~d}), 6.5(\mathrm{VT2}), 22.9\left(\mathrm{VT} 2 \mathrm{~d}_{\mathrm{act}}\right),>1000 \\
\mathrm{ng}(\mathrm{Stx}, \mathrm{VT} 2 \mathrm{~b}, \mathrm{VT2c}) ; \\
\mathrm{ED}_{50}=63 \mathrm{pg} / \mathrm{mL}(\mathrm{Stx}) \text { to } 46.7 \mathrm{ng} / \mathrm{mL}(\mathrm{VT} 2 \mathrm{c}) ; \\
E D_{50}=1.1 \mathrm{pg} / \mathrm{mL}\left(\mathrm{VT} 2 \mathrm{~d}_{\text {act }}\right) \text { to } 32 \mathrm{ng} / \mathrm{mL} \text { (VT2c) }\end{array}$ & $\begin{array}{l}\text { Fuller et al., } \\
2011\end{array}$ \\
\hline Mice (CD-1); & $\begin{array}{l}\text { i.v. or i.p. } L D_{50} \approx 5 \times 10^{-5} \mathrm{mg} / \mathrm{kg}(\mathrm{VT} 2) \text {, greater renal } \\
\text { damage } \\
\text { i.v. or i.p. } L D_{50} \approx 0.02 \mathrm{mg} / \mathrm{kg}(\mathrm{VT} 1) \text {; }\end{array}$ & Tesh et al., 1993 \\
\hline
\end{tabular}




\begin{tabular}{|c|c|c|}
\hline Model* & Toxicity (Toxin) and other information" & Reference \\
\hline $\begin{array}{l}\text { Vero cells; } \\
\text { CFPA (rabbit) }\end{array}$ & $\begin{array}{l}\mathrm{CD}_{50} \approx 50 \mathrm{pg}(\mathrm{VT} 1 / 2) \\
\mathrm{ID} \mathrm{D}_{50} \approx 40 \mathrm{ng} / \mathrm{mL}(\mathrm{VT} 1 / 2)\end{array}$ & \\
\hline $\begin{array}{l}\text { Mice (CD-1 ơ); } \\
\text { Vero cells }\end{array}$ & $\begin{array}{l}\mathrm{LD}_{50}<10 \mathrm{CFU}(\mathrm{VT} 2 \mathrm{~d} \text { VTEC), antibody treatment prevented } \\
\text { renal damage, but not colonization. } \\
\text { VTEC producing VT2a with } 2 \mathrm{c} \text { or } 1 \text { a were not able to kill } \\
50 \% \text { even at oral doses of } 10^{10} \mathrm{CFU} \text {; } \\
\text { VT2d activated by mucus increased toxicity } 1000 \mathrm{X}\end{array}$ & $\begin{array}{l}\text { Lindgren et al., } \\
1993\end{array}$ \\
\hline $\begin{array}{l}\text { Mice (CD-1 o'); } \\
\text { Vero cells; }\end{array}$ & $\begin{array}{l}\text { i.p. } L D_{50} \approx 1-2 \mathrm{ng}(\mathrm{VT} 2 \mathrm{a} \text { and VT2c); } \\
C D_{50} \approx 5 \mathrm{pg}(\mathrm{VT} 2 \mathrm{a}), \mathrm{CD}_{50} \approx 500 \mathrm{pg}(\mathrm{VT} 2 \mathrm{c})\end{array}$ & $\begin{array}{l}\text { Lindgren et al., } \\
1994\end{array}$ \\
\hline Vero cells & $\mathrm{CD}_{50} \approx 3.5 \mathrm{pg} / \mathrm{mL}(\mathrm{VT} 1)$ & $\begin{array}{l}\text { Tam and } \\
\text { Lingwood, } 2007\end{array}$ \\
\hline $\begin{array}{l}\text { Vero cells; } \\
\text { HSVEC; } \\
\text { HUVEC; } \\
\text { HeLa }\end{array}$ & $\begin{array}{l}\mathrm{CD}_{50} \approx 1 \mathrm{pg}(\mathrm{Stx}) ; \mathrm{EC} \mathrm{CD}_{50} \approx 1.4 \times 10^{-8} \mathrm{M}(\mathrm{Stx} \text { and VT2), } \\
\text { 100x lower (VT, }+\mathrm{TNF} \alpha) ; \\
\mathrm{Gb3} \mathrm{nM} / \mathrm{mg:} 0.03 \text { (HSVEC); } 0.06 \text { (HUVEC); } 25 \text { (HeLa); and } \\
80 \text { (Vero) }\end{array}$ & Tesh et al., 1991 \\
\hline $\begin{array}{l}\text { HRMEC; } \\
\text { HUVEC }\end{array}$ & $\begin{array}{l}\mathrm{LD}_{50} \approx 10 \mathrm{fM}(\mathrm{VT} 2), \mathrm{LD}_{50} \approx 10 \mathrm{pM}(\mathrm{VT} 1) ; \\
\mathrm{LD}_{50} \approx 10 \mathrm{nM}(\mathrm{VT} 1), \mathrm{LD}_{50}>100 \mathrm{nM}(\mathrm{VT} 2)\end{array}$ & $\begin{array}{l}\text { Louise and } \\
\text { Obrig, } 1995\end{array}$ \\
\hline HUVEC & $\begin{array}{l}\text { Presence of LPS had a synergistic effect on VT toxicity, } \\
\text { while not toxic itself }\end{array}$ & $\begin{array}{l}\text { Louise and } \\
\text { Obrig, } 1992\end{array}$ \\
\hline HRMEC & 1000 times more sensitive to VT2 than HUVEC & Jacewicz 1999 \\
\hline HRMEC & $\begin{array}{l}10 \mathrm{nM} \text { VT1 caused cytotoxicity in 0-6\% of cells, which } \\
\text { increased to } 76-90 \% \text { when preincubated with TNF } \alpha \\
(10 \mathrm{ng} / \mathrm{mL})) \text { with as little as } 0.1 \mathrm{pM} \text { causing toxicity; TNF } \alpha \\
\text { and other cytokines caused increased binding and Gb3 } \\
\text { synthesis. VT1 slightly more potent than VT2. }\end{array}$ & $\begin{array}{l}\text { van Setten et } \\
\text { al., } 1997\end{array}$ \\
\hline $\begin{array}{l}\text { Yeast; } \\
\text { CFPA rat }\end{array}$ & $\begin{array}{l}\text { VT2A had higher depurination and toxicity compared to } \\
\text { VT1A } \\
\text { same results. }\end{array}$ & $\begin{array}{l}\text { Basu et al., } \\
2016\end{array}$ \\
\hline CFPA rabbit & $\mathrm{IC}_{50}=0.1 \mathrm{nM}(\mathrm{VT} 1 \mathrm{~A})$ & $\begin{array}{l}\text { Brigotti et al., } \\
1997\end{array}$ \\
\hline CFPA & $\begin{array}{l}\mathrm{IC}_{50}=0.8 \mathrm{nM}(\mathrm{VT} 1 \mathrm{~A}) \text { for both prokaryotic (E. coli) and } \\
\text { eukaryotic (Artemia Salinas) ribosomes }\end{array}$ & Suh et al., 1998 \\
\hline
\end{tabular}

* CFPA - Cell-free protein assays; A method of determining the direct effect on ribosomes by measuring protein synthesis products

${ }^{\#} \mathrm{CD}_{50}$ - median cytotoxic dose; i.p. - intraperitoneally; i.v. - intravenous; $I \mathrm{I}_{50}$ - median inhibitory concentration; $\mathrm{ID}_{50}$ - median inhibitory dose; $\mathrm{LD}_{50}$ - median lethal dose; w.o. - weeks old

Sperandio, 2012; Zoja et al., 1992). Baboon models with intravenous injection of VT

have provided a much clearer understanding of renal pathology in humans and have 
shown some intestinal damage at higher doses. However, although baboons may have similar levels of Gb3 in the kidney and gastrointestinal tract, prospective ingestion studies where only a small percentage of animals show the full range of symptoms would be impractical due to the expense (Andreoli et al., 2002). Other animal models have also been used with similar drawbacks (Andreoli et al., 2002). As such, patients already infected with VTEC must be studied for more accurate information. Cellular models have also proved useful. Some of the toxicologic data is summarized in Table 1.

Overall, the data suggests that both VT1 and VT2 have similar molecular toxicities. Different cell lines are more susceptible than others to VT, but VT2 is much more toxic to an organism on the whole, with subtypes playing some role in higher toxicity. Some of the factors that point to more severe disease in humans caused by VT2 will be further discussed in Section 1.1.6.

\subsubsection{Verotoxin Subtypes and Disease Association}

Although the subtypes of VT are similar in structure and share a high degree of sequence homology, certain subtypes have a higher association with disease in humans. A comprehensive study in 2012 was performed leading to a standardized naming system for VT subtypes based on sequence phylogeny. Additionally, a polymerase chain reaction (PCR) protocol for toxin subtyping was created and validated to ensure future variants are properly typed (Scheutz et al., 2012). VT1 and VT2 share about 56\% sequence identity (Jackson et al., 1987), with subtypes (1c, 1d; and 2b, 2c, 2d, 2e, 2g) being $>90 \%$ similar to the prototypes VT1a and VT2a. VT2f, however, is only about $60 \%$ 
identical to VT2a (63.4 and $57.4 \%$ sequence identity for A and B subunits) (Schmidt et al., 2000). VT variants are also frequently found with minor amino acid differences from the prototypic forms (Scheutz et al., 2012).

In general, VT2 is more frequently found in VTEC related illness and outbreaks. It is more strongly associated with HUS than VT1 (Boerlin et al., 1999; Ostroff et al., 1989). The various subtypes display differing association with severe patient outcome with VT2a, VT2c, and VT2d most commonly associated with HC and HUS (Orth et al., 2007; Bielaszewska et al., 2006).

VT2a has been found to be significantly associated with HUS and eae genes (a set of virulence factors involved in colonization of VTEC) and produced significantly higher amounts of toxin compared to other VT2 subtypes (Kawano et al., 2012; Beutin et al., 2004; Friedrich et al., 2002).

VT2b is typically associated with mild disease (Piérard et al., 1998). VT2b had previously been named $2 d$ until 2012 when the sequences were compared (Scheutz et al., 2012). Of the VT2 subtypes VT2b binds with the lowest affinity to receptors, suggesting an explanation for lower toxicity (Karve et al., 2014). It was also not associated with eae genes and has a low cytotoxicity (Piérard et al., 1998).

VT2c, compared to 2 a, is statistically associated with HUS although strains tested have been reported to produce less toxin and patients had a lower risk of developing HUS (Kawano et al., 2012; Friedrich et al., 2002). It is also frequently produced in combination with other VT subtypes and associated with eae (Orth et al., 2007). VT2d, uniquely, can be activated by elastase found in intestinal mucus leading to 
increased toxicity (Melton-Celsa et al., 1996; Lindgren et al., 1993). It is suggested that two genetic motifs shared by the subtype --at the end of the A subunit and beginning of the B subunit-- are responsible for the activatable property (Scheutz et al., 2012). Generally, VT2d is not associated with eae strains (Orth et al., 2007; Beutin et al., 2004; Friedrich et al., 2002). However, $2 d$ strains have been seen to be associated with severe disease outcome (Bielaszewska et al., 2006). VT2d strains are less commonly reported than VT2a and 2c (Friedrich et al., 2002).

VT2e is rarely associated with human disease, but primarily with pig edema disease (Scheutz and Ethelberg, 2008; Frydendahl, 2002). VT2e has differences in the B subunit causing a binding preference for Gb4 receptors, rather than Gb3 (Ling et al., 2000). This contributes to the host specificity. In humans, VT2e was associated with uncomplicated infection (Friedrich et al., 2002) and is less commonly reported (Beutin et al., 2004) although there have been some HUS cases (Franke et al., 1995).

VT2 $\mathrm{f}$ has the most different sequence of the VT2 subtypes. Due to this $2 \mathrm{f}$ is frequently not detected by commercial PCR or serological test kits (Feng et al., 2011). $2 f$ was classified as a new subtype by Schmidt et al. in 2000 when isolated from five pigeons, however an isolate tested in that study (H.I.8) had been recovered previously from infant diarrhea (Konowalchuk, 1977) and reported as VT2 variant by Gannon et al. (1990). Additionally, $2 \mathrm{f}$ strains tested have low toxin production, generally requiring mitomycin induction to be detected (Schmidt et al., 2000). Although $2 \mathrm{f}$ genes were found in $12.5 \%$ of pigeon droppings (Dell'Omo et al., 1998) $2 \mathrm{f}$ is rarely found in human isolates (Friedrich et al., 2002; Orth et al., 2007). A study in the Netherlands found VT2f 
is associated with mild disease (Friesema et al., 2014).

VT2g subtype was most recently discovered (Leung et al., 2003). A number of strains have been identified from cattle, wastewater, aquatic environments and humans, however the role in human illness has not yet been investigated (Granobles Velandia et al., 2012; Beutin et al., 2007). HeLa and Vero cell cytotoxicity, however, is comparable to other VT2a strains (Granobles Velandia et al., 2012).

Comparatively, VT1 strains have a lower association with severe disease outcomes and are often more associated with strains isolated from animals (Blanco et al., 2004b; Orth et al., 2007). VT1c is associated with sheep isolates, the absence of eae, the presence of other adhesin genes and hemolysin, the presence of other VTs, and mild disease (Beutin et al., 2004; Friedrich et al., 2003). VT1d isolates have not yet been reported and their role as agents of human disease is not clear (Beutin et al., 2007).

Although priority detection would be directed towards strains expressing VT2a and $2 \mathrm{c}$ due to a higher association with severe disease, no VTEC expressing other subtypes should be disregarded as non-pathogens even if they have yet to be identified in human illness.

\subsubsection{Factors Implicated in Higher Disease Severity for VT2}

Aside from production of VT other factors contribute to the development of severe symptoms. There have been a number of studies to identify some of the potential risk factors which contribute to VT2 being more strongly associated with severe patient outcomes, which are briefly discussed below. 
It has been suggested that VT2 induces a greater expression of signalling molecules, leading to more severe disease progression in humans. VT1 and VT2 were shown to have the same level of toxicity and that $A$ and $B$ subunits did not induce the expression on their own (Brandelli et al., 2015). However, in macrophage-like THP-1 cells treated with VT2, 14 of the 40 cytokines or chemokines monitored showed significantly increased levels including pro-inflammatory, immune cell proliferating, growth-promoting factors and other chemokines (Brandelli et al., 2015). These factors have been associated with HUS suggesting that VT2 may play a greater role than VT1 in enhancing macrophage infiltration and fibrin deposition (Brandelli et al., 2015; Fu et al., 2004). Also, both TNF-a and IL-1 $\beta$, as well as a few other cytokines to a lesser extent, induce $\mathrm{Gb}_{3}$ expression and may contribute to greater impact of the toxin at target organs (van der Kar et al., 1992).

Another potential factor is the subunit stability and activity. VT1 and VT2 B subunit pentamers have a very different stability with VT2 pentamers being less stable. This is caused largely by replacement of a leucine with glutamine at amino acid residue 41 in VT1 (Karve et al., 2014; Conrady et al., 2010). It has been suggested that this glutamine, by destabilizing the B pentamer, increases selectivity for Gb3, largely preventing off-target binding (Karve et al., 2014). In comparison, VT1 has been found to bind lung tissue while VT2 only binds to target tissues (Rutjes et al., 2002). The added stability of VT2 holotoxin may help target high density Gb3 areas (Tesh et al., 1993). HRMEC (human renal glomerular epithelial cells) for example had an $\mathrm{LD}_{50}$ of about 10 fM with VT2 which is about 10 molecules of toxin per cell while the $L D_{50}$ was about 10 
pM for VT1. HUVEC (human umbilical vein epithelial cells) a non-target cell type in humans, however, were more sensitive to VT1 (LD $50 \sim 10 \mathrm{nM}, \mathrm{VT2}>100 \mathrm{nM})$ (Louise and Obrig, 1995). There is also some evidence, that compared to VT1, the A subunits of VT2 show both a higher level of affinity for ribosomes and higher catalytic activity in yeast and mammalian ribosomes (Basu et al., 2016). So potentially both A and B subunits contribute to the higher association of VT2 with severe disease.

Finally, in general, VT2 (especially 2a, 2c, and 2f) has a greater association with a greater number of virulence factors like intimin, hemolysin, and others (Franz et al., 2015; Werber et al., 2003). These associations appear to be with sets of virulence factors resulting in related virulence profiles (Franz et al., 2015). This, however, may be a compounding effect. VT2 strains also tend to produce higher levels of toxin (Ritchie et al., 2003) than VT1 strains, which would also aid in severe disease progression.

\subsection{Verotoxin-producing Escherichia coli (VTEC)}

\subsubsection{General Information}

Escherichia coli (E. coli) are Gram-negative, rod-shaped bacteria, typically found as commensal organisms of the gastrointestinal tracts of warm-blooded animals, including humans. They are oxidase-negative, facultatively anaerobic, can be motile with peritrichous flagella, and have a maximum growth rate at $37^{\circ} \mathrm{C}$ (Croxen et al., 2013). However, individual strains of E. coli can vary significantly in their phenotypic characteristics. Seven main groups (pathotypes) of $E$. coli that cause enteric illness in humans exist. Verotoxin-producing E. coli (VTEC) (including enterohemorrhagic E. coli 
[EHEC]) is one of those pathotypes. The pathotypes are distinguished by the possession of specific virulence factors and the mechanisms by which they induce disease (Croxen et al., 2013). VTEC strains are distinguished by the production of at least one verotoxin (VT). The VT genes are encoded on a mobile genetic element, the stx-phage, thus VTEC strains are not necessarily clonally related (Schmidt, 2001).

Isolates of E. coli are frequently differentiated by a serological scheme based on antibody reactions to three classes of cell surface antigens, $\mathrm{O}, \mathrm{K}$ and $\mathrm{H}$ (Kauffmann, 1947). The $O$ antigen is the polysaccharide portion of lipopolysaccharides (LPSs) found in the outer cell membrane. The $\mathrm{K}$ antigens are diverse capsular polysaccharides. The $\mathrm{H}$ antigen is the flagellin protein, which composes the flagellar whip (Scheutz et al., 2004). However, few laboratories are equipped to test $\mathrm{K}$ antigens, so generally only the $\mathrm{O}: \mathrm{H}$ serotype, for example 0157:H7, is used to type isolates (DebRoy et al., 2011). Serotyping can also be performed at the molecular level by polymerase chain reaction (PCR) for genes involved in synthesis of the antigens (Wang et al., 2003; DebRoy et al., 2011). Currently 185 O-types and $53 \mathrm{H}$-types have been identified with many of the associated genes sequenced (DebRoy et al., 2016; Chui et al., 2015; Scheutz et al., 2004). Many strains are also found that do not express LPS or flagellin resulting in $O$ rough or non-motile (NM) designations, respectively. Many of the O rough or NM types are a result of point mutations in the corresponding $\mathrm{O}$ or $\mathrm{H}$ genes which can often be detected by PCR (Beutin et al., 2004). There are also strains that do not react with that standard set of antibodies, resulting in an untypable classification. Although some serotypes are more common than others among certain pathotype isolates, the majority 
of $E$. coli virulence factors, including VT, are not linked to serotype (Levine, 1987), with the serotype antigens themselves not a cause of virulence (Friedrich et al., 2002).

In the early 1980s, not long after the discovery of VT, an association of VTEC infections with severe illness, both hemorrhagic colitis (HC) and hemolytic uremic syndrome (HUS), was observed (Riley et al., 1984; Karmali et al., 1983). In both the original outbreaks, strains of $0157: \mathrm{H} 7$ were isolated, and have been observed since to regularly cause outbreaks with cases of severe illness (Bavaro, 2012). Since O157:H7 was among the first reported VTEC serotypes and frequently caused severe illness it became the focus of VTEC detection and prevention. 0157:H7 strains do not commonly ferment sorbitol and are $\beta$-glucuronidase negative, traits which form the basis of differential media for the detection and isolation of this serotype (March and Ratnam, 1986). The frequent association between 0157:H7 and a small number of serotypes with clinical HC or HUS cases led to the proposal of the classification of enterohemorrhagic E. coli (EHEC). This subgroup was defined by its production of severe clinical symptoms, frequent reports of sporadic or outbreak cases, production of at least one type of VT, enterohemolysin (encoded on a 60-MDa plasmid by the EhxA gene), and production of attaching and effacing lesions (later linked to genes encoded at the locus of enterocyte effacement [LEE] (McDaniel et al., 1995)) as well as a couple other minor differences from other pathotypes (Levine, 1987). E. coli O157:H7 was the most commonly reported serotype, possibly due to ease of isolation augmenting its status as the primary illnesscausing serotype of VTEC (Goldwater and Bettelheim, 1996), and this serotype remains the most commonly reported VTEC serotype in Canada and the United States (Thomas 
et al., 2013; Scallan et al., 2011). Significantly more reporting of 0157 strains in VTECrelated illness has led to the broad categorization of VTEC into 0157 and non-0157 strains.

The non-0157 group, however, is highly diverse in phenotype, genotype, as well as association with outbreaks and patient outcomes. Over 470 non-0157 serotypes have been isolated from human infections (Blanco et al., 2004a). Non-0157 VTEC as a group have no phenotypic characteristic which could be used to differentiate them as a subpopulation from other $E$. coli and as such are difficult to select for, isolate, and monitor (Gill et al., 2012).

\subsubsection{Epidemiology of VTEC}

VTEC can be transmitted through direct contact with infected people, animals, or water, but exposure in industrialised nations is more frequently associated with consumption of contaminated food (Gyles, 2007). As such VTEC are primarily considered foodborne pathogens. As with non-pathogenic $E$. coli strains, diverse strains of VTEC are carried in the gastrointestinal tract of many animals, especially ruminants, and can be isolated from a variety of environmental sources (Mauro and Koudelka, 2011). VTEC rarely cause disease in host animals and represent a small number of the coliform bacteria present (Beutin et al., 1993). Cattle lack the VT receptor, Gb3, in their intestinal tissues which prevents a disease process similar to humans from occurring (PruimboomBrees et al., 2000). However, VTEC expressing VT2e cause edema disease in pigs (Frydendahl, 2002). Clinically important strains represent only a subset of VTEC found in 
animals (Blanco et al., 2004b).

Due to the common colonisation of cattle, there has been a strong association of VTEC with beef and dairy products. Initial contamination with E. coli can happen anywhere along the production line with a wide variety of serotypes isolated (Gill and Gill, 2015). The contamination can be from the animal's own bowel, material from other animals in the production process, from machinery, and from workers (Stephan and Untermann, 1999). Contaminated meat (especially ground beef) and unpasteurized milk are among the most common food products associated with VTEC illness (Karch et al., 2005). A study in the United States over a 24-month period with samples from 18 ground beef producers found a mean prevalence of $24.3 \%$ for VT genes, with non-0157 isolates from at least 42 O-groups (Bosilevac and Koohmaraie, 2011). The prevalence of VTEC in American and Canadian cattle and beef products as well as intervention strategies is further reviewed in Gill and Gill, 2012.

In addition to meat products, fresh produce is the next most commonly reported food associated with VTEC outbreaks. VTEC can contaminate water, especially through farm runoff into local wells or groundwater which can directly infect consumers (BGOSHU, 2000). Through contaminated irrigation water VTEC can also contaminate produce. Produce is additionally exposed to common bacterial contamination routes such as: animals, workers, processing machinery, and cross-contamination in meal preparation (Bavaro, 2012). Due to frequent consumption of raw produce it is a food commodity with significant risk of infection if contaminated. Foods which have been associated with VTEC include, but are not limited to: meat products, dairy products, 
fresh or processed produce, fruit, juices, and processed foods (Catford et al., 2014; CDC, 2014; Gyles, 2007).

To reduce the prevalence of VTEC, various food treatment methods can be used to inactivate bacteria and increase safety without greatly increasing expense.

Treatments involving heat treatment, high pressure processing, UV treatment, and applications of antimicrobial chemicals, pasteurization, and combinations thereof are reviewed in Bavaro (2012) and Gill and Gill (2012). VTEC, however, have very low infectious doses relative to other $E$. coli pathotypes (Croxen et al., 2013). The infectious dose for some strains has been estimated to be below a hundred cells (Tuttle et al., 1999; Tilden et al., 1996; Bell et al., 1994), with a significant risk of infection from exposure to a single cell (Teunis et al., 2004). Recent outbreaks in Canada have involved food with VTEC most-probable-number (MPN) levels as low as 2.2 MPN/100g in beef patties, and 0.37 to 0.95 MPN/100g in Gouda cheese (Gill and Huszyczynski, 2016; Gill and Oudit, 2015).). Additionally, VTEC have been shown to have household secondary transmission rates between 4-16\% (Parry and Salmon, 1998) or potentially as high as $26 \%$ (Ludwig et al., 2002) which is likely only possible due to the low infectious dose.

Adding to the ability of VTEC to survive and further contaminate or infect they have also been observed to: produce viable but nonculturable (VBNC) cells (Dinu and Bach, 2011; Liu et al., 2010); survive in the environment for months after contamination (Varma et al., 2003; Hancock et al., 1998); and survive both low pH and low water environments (Nguyen and Sperandio, 2012; Tilden et al., 1996; Hathcox et al., 1995; Glass et al., 1992). Additionally, 0157 and other pathogenic serotypes were found to 
have a high rate of mutation compared to control strains (LeClerc et al., 1996)

undergoing frequent genetic rearrangements which may lead to better adaptation and acquisition of virulence factors, allowing them to survive in so many stressful situations.

VTEC is an important cause of foodborne illness worldwide causing both sporadic cases and outbreaks. It has been estimated that 175,000 illnesses ( $64 \%$ non-0157), including more than 2400 hospitalizations (11\% non-0157) and 20 deaths (no non0157) are caused by VTEC infections in the United States annually (Scallan et al., 2011). VTEC caused 29 confirmed outbreaks in 2012 (83\% 0157) causing 98 hospitalizations and 3 deaths (CDC, 2014). Similarly, in Canada, it has been estimated that foodborne illness caused by VTEC occurs at a rate of about 102 cases per 100,000 people (about 33,000 illnesses per year) with about $62 \%$ of cases being caused by non-0157 VTEC (Thomas et al., 2013). These estimates make VTEC the 6 th most frequent cause of domestically acquired foodborne illness in Canada (Thomas et al., 2013). Globally, the World Health Organization estimated that in 2010 about 1.2 million illnesses, 128 deaths, and 13000 disability adjusted life years (years of life lost or years lived with disability) were caused by VTEC (WHO, 2015).

The cost of illness caused by 0157 strains was estimated to be about $\$ 405$ million USD for the US in 2003. Of this a majority was caused by premature death (especially of children), with $\$ 30$ million in health care and $\$ 5$ million in lost productivity (Frenzen et al., 2005). This number was based on approximately 74,000 cases per year caused by 0157 . Similar estimates of annual 0157 illness have been estimated, with the addition of 112,000 illnesses caused by non-0157 strains (Scallan et al. 2011). As non- 
O157 illness is less likely to cause severe illness the economic impact remains similar. In Canada where estimates of cases are lower (approximately 13,000 0157 and 20,000 non-0157 cases) the estimate cost is around \$115 million CAD per year (Thomas et al., 2013). The combination of risks of severe illness as well as economic expense place detection of all VTEC strains as a high priority.

Although $\mathrm{O} 157$ is the most common O-type of VTEC reported as a cause of illness, a wide diversity of VTEC serotypes that cause illness exist with geographic distribution varying considerably from region to region (Johnson et al., 2006). This diversity of serotypes and frequency in the European Union, United States, Canada, and other select regions is summarized in Catford et al. (2014). In Canada between 1998 and 2012 the six most common typeable non-0157 in order were: 026; 0121; 0103; 0111; 0145; and 0117 which were responsible for $52 \%$ of reported and typed illness. Another 63 O-types were reported during this period and $10.8 \%$ of strains reported were rough or untypable, representing the $4^{\text {th }}$ most common grouping (Catford et al., 2014). Further to the diversity of O-types that cause illness, a review by Bettelheim (2007) identified 91 different O-types isolated in at least three cases of illness, with a total of 162 O-types reported as clinical isolates. As with $0157: \mathrm{H7}$, the $\mathrm{O}$ types more important in human disease have a greater association with ruminants (Bettelheim, 2007).

\subsubsection{Verotoxin Production, Expression, and Virulence Factors}

VT is encoded by vtx genes (also referred to as stx genes). They are acquired through horizontal gene transfer by lysogenic lambdoid bacteriophages, called stx- 
phages (reviewed in Schmidt, 2001). VTEC can carry multiple sets of $v t x$ genes with some strains found to have three or four different types (Scheutz et al., 2012; Orth et al., 2007). Usually, phage infection will result in production of phage particles and lysis. The phage may instead undergo lysogeny and integrate its genes into the chromosomal DNA of the E. coli converting it into a VTEC (Karch et al., 1999).

As part of the phage genes, toxin production is regulated through multiple transcription termination and antitermination processes (Neely and Friedman, 1998). The toxin is encoded in the late genes of the phage genome, so VT is only upregulated during the lytic cycle (Mühldorfer et al., 1996). Stress conditions which induce the phage Iytic cycle cause the bacteria to produce increased amounts of both phage particles and toxin (Gyles, 2007; Schmidt, 2001). One source of such stress is the presence of antimicrobial agents. This is due to the bacterial SOS response which induces the phage's lytic cycle by cleavage of termination proteins (Mauro and Koudelka, 2011; Schmidt, 2001). Upregulation results in increasing gene copy number, gene promoter activity, and release of VT (Gyles, 2007; Neely and Friedman, 1998). A detailed description of the late gene cascade is presented in Mauro and Koudelka (2011). Aside from production of high levels of VT by a population of VTEC under stress conditions, VT production occurs due to spontaneous induction of the lytic cycle, which may be higher in more virulent strains (Neupane et al., 2011).

There are a number of factors that affect the expression level of VT. Some of these are summarized in Table 2. Generally, these factors either affect general growth of VTEC, induction of the lytic cycle, or expression of the VT genes. 
Table 2 - Some factors affecting expression of VT

\begin{tabular}{|c|c|c|}
\hline Factor & Effect on VT production & Reference \\
\hline Glucose & $\begin{array}{l}\text { Slightly decreased expression of VT associated } \\
\text { genes; } \\
\text { Increased toxicity of VT2e strains }\end{array}$ & $\begin{array}{l}\text { Delcenserie et al., 2011; } \\
\text { MacLeod and Gyles, } 1989\end{array}$ \\
\hline $\begin{array}{l}\text { E. coli broth } \\
\text { medium }\end{array}$ & $\begin{array}{l}\text { Higher VT production compared to other media } \\
\text { (BHI, Evans, LB, Penassay, BPW, Syncase, TSB), } \\
\text { likely caused by bile salts }\end{array}$ & Rocha and Piazza, 2007 \\
\hline Iron & $\begin{array}{l}\text { Repressed VT1 under high levels by the Fur } \\
\text { protein (the promoter region is homologous to } \\
\text { other Fur regulated genes); no effect on VT2 }\end{array}$ & $\begin{array}{l}\text { Calderwood and } \\
\text { Mekalanos, 1987; } \\
\text { Muhldorfer et al., } 1996\end{array}$ \\
\hline Nitric Oxide & $\begin{array}{l}\text { Reduced VT expression by blocking the SOS } \\
\text { response pathway }\end{array}$ & Vareille et al., 2007 \\
\hline Preservatives & Decreased lytic activity and phage titer & Subils et al., 2012 \\
\hline Mitomycin & $\begin{array}{l}\text { Increased VT by activation of SOS response; } \\
50 \mathrm{ng} / \mathrm{mL} \text { overnight increased VT2 production } \\
\text { to } 2,528 \mathrm{ng} / \mathrm{mL} \text { ( } 36.11 \mathrm{nM}) ; 250 \mathrm{ng} / \mathrm{mL} \text { for } 6 \\
\text { hours increased VT2 10- to } 140 \text {-fold yielding } \\
3000-100000 \mathrm{ng} / \mathrm{mL} \text { (42.55-1418.4 nM) }\end{array}$ & $\begin{array}{l}\text { Hull et al., 1993; MacLeod } \\
\text { and Gyles, 1989; } \\
\text { Mühldorfer et al., 1996; } \\
\text { de Sablet et al., } 2008\end{array}$ \\
\hline $\begin{array}{l}\text { Growth } \\
\text { temperature }\end{array}$ & $\begin{array}{l}\text { Increased VT at higher temperatures, maximum } \\
\text { yield at } 37^{\circ} \mathrm{C}\end{array}$ & $\begin{array}{l}\text { Palumbo et al., 1995; } \\
\text { MacLeod and Gyles, } 1989\end{array}$ \\
\hline Cell count & $\begin{array}{l}\text { Total amount of VT proportionately increased } \\
\text { with cell count }\end{array}$ & Palumbo et al., 1995 \\
\hline Quorum sensing & $\begin{array}{l}\text { Increased production of VT through the luxS } \\
\text { gene which leads to activation of the bacterial } \\
\text { SOS response }\end{array}$ & $\begin{array}{l}\text { Pacheco and Sperandio, } \\
\text { 2012; Yang et al., } 2014\end{array}$ \\
\hline $\begin{array}{l}\text { Incomplete } \\
\text { phage genome }\end{array}$ & $\begin{array}{l}\text { Reduced levels of VT2 production, may not be } \\
\text { able to induce lytic expression }\end{array}$ & Dowd and Williams, 2008 \\
\hline $\begin{array}{l}\text { Spontaneously } \\
\text { Inducing Phages }\end{array}$ & Increased amounts of VT & de Sablet et al., 2008 \\
\hline Toxin Subtype & $\begin{array}{l}\text { Higher } v t x \text { mRNA and VT levels for VT2a } \\
\text { compared to VT2c, even when the same } \\
\text { serotype }\end{array}$ & Kawano et al., 2012 \\
\hline
\end{tabular}

The vtx genes encode the two subunits, $A$ and $B$, sequentially in the phage DNA.

Transcription of the genes is encoded by a single operon with two ribosome binding sites, the stronger being directly upstream of the B subunit to allow more frequent translation (Habib and Jackson, 1993). The subunits have signal peptides which promote 
their secretion into the periplasmic space for proper folding (Jackson et al., 1987). VT is primarily secreted with phage particles through viral cell lysis. The subunits can then combine to produce toxicity (Pellino et al., 2016).

Bacterially released phages can infect host $E$. coli and some other Enterobacteriaceae present (Herold et al., 2004) causing lysis releasing additional toxin and phage particles (Gamage et al., 2003; James et al., 2001; Acheson et al., 1998). The VT phage is very efficient at transduction of other E. coli strains to produce new VTEC. Experiments in vitro or in murine coinnoculation models showed that with susceptible acceptor strains a significant increase of phage particles and toxin were observed (Gamage et al., 2003). Transduction can also take place in sheep (Cornick et al., 2006). Although VT production is the primary virulence factor found in all VTEC, however, strains have been isolated from asymptomatic individuals, so VT cannot be the sole factor in causing disease (Stephan and Untermann, 1999). Other known or putative virulence factors are variably present in VTEC strains and their presence likely influences the strains pathogenicity (Franz et al., 2015; Johnson et al., 2006).

The virulence factors with highest association to enhancing severe VTEC outcomes are VT2 and the LEE (locus of enterocyte effacement) pathogenicity island. The LEE pathogenicity island encodes the genes for intimin (eae gene) and other factors that together assist in effacement and attachment of cells to the intestine (Nguyen and Sperandio, 2012; Werber et al., 2003; Boerlin et al., 1999). LEE is frequently found in 0157 and VT2 carrying strains (Ritchie et al., 2003; Friedrich et al., 2002). However, the German outbreak in 2011 which resulted in over 3956 reported cases (896 HUS and 56 
deaths) involved a strain which was LEE negative and instead possessed genes involved in enteroaggregative $E$. coli (EAEC) virulence including aggR (Bielaszewska et al., 2011; Frank et al., 2011).

Other putative virulence factors include: molecules associated with adhesion, other toxins, flagellar proteins, and more. However, due to a lack of animal models and the potential that these factors contribute only slightly to pathogenicity makes it difficult to determine their impact on virulence (Gyles, 2007). Strains within the same serotype can vary greatly in the number of virulence factors present (Franz et al., 2015). Higher production of VT is also considered to be a potential factor in pathogenicity, with isolates from HUS cases often producing higher levels than those isolated from animals, environmental sources, or humans with mild disease (Zangari et al., 2014; Neupane et al., 2011; de Sablet et al., 2008; Ritchie et al., 2003).

Currently, it is generally accepted that the best indication of potential risk of severe disease is the expression of VT2a or VT2d in strains that are eae (intimin) or aggR (EAEC regulator) positive. Other strains, although potentially virulent, are less likely to cause severe illness (Scheutz, 2014).

\subsubsection{Colonization and Initiation of Illness}

Upon ingestion, VTEC makes its way to the intestines where it can colonize and interact with host cells (Croxen et al., 2013). LEE-positive cells produce factors resulting in intimate adhesion by inducing host-signal transduction producing attaching and effacing lesions (Nguyen and Sperandio, 2012; McDaniel et al., 1995). Non-LEE VTEC 
may interact with host mucosa and intestinal epithelial cells by a variety of other adhesin factors (Farfan and Torres, 2012). These factors assist in formation of biofilms, host cell adherence, bacterial aggregation, and binding to the host mucosa. The bacteria can then multiply, and stx-phage induction can occur in a portion of the population, with resultant VT production and lysis. VT can then interact with intestinal cells or be translocated to the bloodstream to interact with other target cells.

\subsubsection{VTEC Associated Illness and Treatment}

VTEC infection can result in a range of outcomes from asymptomatic carriage (Ludwig et al., 2002), self-limiting watery diarrhea, bloody diarrhea or hemorrhagic colitis (HC), hemolytic uremic syndrome (HUS), and may result in neurological complications, and death. Upon infection with $\mathrm{O} 157: \mathrm{H7}$, the incubation period ranges from 1-12 days (usually about 3 days) before symptoms are seen (Tarr et al., 2005; Mead and Griffin, 1998). Most patient outcomes are self-limiting with recovery within a week. Abdominal cramping and non-bloody diarrhea typically occur first and subsequently about $90 \%$ of patients develop some degree of bloody diarrhea within 1-3 days (Tarr et al., 2005). Frequently, vomiting is observed and some experience fevers (Mead and Griffin, 1998). Difference in rates of progression to HUS have been observed between sporadic cases and outbreaks with about $3-7 \%$ and $20 \%$ or more, respectively (Frank et al., 2011; Tarr et al., 2005; Mead and Griffin, 1998). Progression to HUS occurs about 7 days after initial symptoms or 4 days after bloody diarrhea (Bitzan, 2009). HUS is defined by three main features: microangiopathic hemolytic anemia 
(death of red blood cells, especially in small blood vessels), thrombocytopenia (low levels of platelets), and acute nephropathy (kidney disease or damage) (Siegler and Oakes, 2005). HUS can have other causes, but $90 \%$ of childhood cases are caused by VTEC (Siegler and Oakes, 2005). Of patients that develop HUS about 9\% die (mostly during the acute phase of illness) with another 3\% later developing end-stage renal disease (Garg et al., 2007). An additional 25\% of patients develop additional long-term renal sequelae: proteinuria (15\%); hypertension (10\%); or low glomerular filtration rates (16\%) (Garg et al., 2007). Severe infections can result in damage to the nervous system (Magnus et al., 2012; Nathanson et al., 2010), intestinal necrosis, brain infarction, pancreatitis or pseudocysts, and exocrine dysfunction (Siegler and Oakes, 2005).

Generally, infants, children, and the elderly are more susceptible, but VTEC infections affect people of all ages (Catford et al., 2014). VTEC is the leading cause of acute renal failure in children globally. $3-18 \%$ of VTEC infections of children progress to HUS, with a mortality rate of 1-4\% (Bitzan, 2009). Elderly individuals are the most likely to die after developing HUS (Gould et al., 2009).

Other potential factors in the progression of severe disease are postulated. Persons with higher expression of VT receptor (Gb3) on red blood cells (Pk blood group) may be less susceptible to development of HUS (Newburg et al., 1993). While the availability of commensal gut flora susceptible to VT phage may promote severe outcome, due to higher levels of VT production (Gamage et al., 2003). VT subtypes are associated with different patient outcomes (discussed in Section 1.1.5).

Currently, there is no known cure for HUS, and antibiotic treatment is believed 
to have no impact or to potentially worsen symptoms due to enhanced VT production resulting from phage induction (Agger et al., 2015; Wong et al., 2000). The bulk of therapy consists of monitoring fluid and electrolytes, providing nutritional support, treatment of hypertension, providing blood transfusion when necessary, and dialysis (Siegler and Oakes, 2005; Tarr et al., 2005). About half of all HUS patients require dialysis, primarily for severe electrolyte or acid-base imbalances, uremic symptoms or fluid overload failing to respond to other treatment (Bitzan, 2009). Following dialysis, kidney function often recovers after 5-7 days with mortality rates of HUS patients around 3-5\% (Siegler and Oakes, 2005). Additionally, there is potential benefit for survivors to take angiotensin-converting enzyme (ACE) inhibitors to help reduce the chances of hyperfiltration related renal injury or the development of end-stage renal disease (Van Dyck and Proesmans, 2004). Volume expansion therapy has also shown some protection to the kidneys and to reduce the severity of HUS (Ake et al., 2005). Aside from supportive treatments, other intervention strategies are being investigated (Pacheco and Sperandio, 2012). Although antimicrobials have mixed results in literature, given confirmation of some criteria (fecal absence of HUS-related strains; relevant clinical details; absence of kidney disease; and patient hydration) it may be considered safe to use protein synthesis inhibiting agents or other non-DNA damaging antimicrobials (Agger et al., 2015; Ichinohe et al., 2009). Treatment of asymptomatic carriers with antimicrobials has also been performed effectively (Jensen et al., 2005). Treatment with subinhibitory concentrations of antimicrobials can cause an increase in VT production so caution is required (Naasar et al., 2013). Treatment with toxin 
neutralizing antibody may be useful in early stages of disease, however, this requires early detection to administer in a timely manner and may result in an immune response (Bitzan et al., 2009; Siegler and Oakes, 2005). VT receptor mimics attached to a solid support such as silica have also been developed (Kitov et al., 2000). Immunization for certain VTEC strains seems highly impractical (Bitzan, 2009; Ludwig et al., 2002). Other creative means of blocking the receptor-binding competitively or providing immunity have been proposed. For all new treatment options, clinical trial testing presents major challenges due to relatively few patients and rapid disease stages for controlled administration of treatments (Bitzan, 2009). Likely the best measures to reduce illnesses are preventative measures such as hygiene and education (Seto et al., 2007) and hygienic food processing to minimize exposure.

\subsubsection{Analytical Methods}

Analysis for VTEC always starts with an enrichment procedure. A sample is suspended in a liquid media favourable for growth and incubated at an appropriate temperature. This allows any VTEC present to multiply, while the inclusion of selective agents can inhibit the growth of other bacteria present (Gill et al., 2012).

Following enrichment most methods use a PCR or serological assay to screen enrichment media samples for the presence of the vtx genes or VT. This allows identification of positive samples for further isolation and the exclusion of negative samples (Feng et al., 2011). Some samples will test positive for PCR, and not serological tests, with the opposite true of other samples (Beutin et al., 2002). Aside from true 
negative samples, certain limitations apply to both methods. A comparison found that PCR analysis was generally better at determining the presence of the various subtypes, while still missed some less important in clinical disease (1d and 2f) (Feng et al., 2011). Differing sensitivities, antibody affinity, and strain-to-strain variation could produce false negative results from serological tests (Feng et al., 2011). Unfortunately, PCR does not differentiate between dead and living cells (or VTEC and other VT-producing bacteria) and can lead to false positives unless paired with an isolation method (Jin et al., 2008). Isolation can be conducted concurrently to screening and is typically performed by plating an aliquot of enrichment media onto solid media to isolate individual colonies. Generally, isolating VTEC can be challenging as their colonies are not morphologically distinct from other $E$. coli resulting in the need to individually screen a large number of colonies to confirm VT production or vtx genes (Gill et al., 2012). Isolation of classical 0157:H7 is relatively simple since it does not metabolize sorbitol, allowing colony differentiation by sorbitol fermentation (March and Ratnam, 1986). However, such media do not differentiate non-O157 VTEC which could lead to falsely attributed disease in mixed cultures (Goldwater and Bettelheim, 1996) or false negatives. Unfortunately, there is no biochemical marker that is distinct to all VTEC. Identification of isolates without differentiation is highly time consuming and laborious (Chui et al., 2011). As such there is a need for a quick screening method or high throughput methods (Mead and Griffin, 1998). Agars which claim to differentiate potentially pathogenic $E$. coli are commercially available; however, most do not allow growth of a full diversity of VTEC, and if they do the process of confirmation is still 
lengthy due to minimal differentiation (Gill et al., 2014).

Isolates are then characterized to confirm the species identity and the presence of virulence associated characteristics. Characterization may include serotyping, molecular phylogeny, or PCR for selected genes (DebRoy et al., 2011). Typically serotypes and virulence factors tested for are those ranked as highest priority. Relative expense and complexity makes testing for a full set of serotypes difficult (Bettelheim, 2007). Many characterization methods are being replaced by whole genome sequencing since the techniques and instruments have become less expensive, more accessible, and offer faster typing (Joensen et al., 2014). Searching the genome for any of the related gene sequences can be done all at once without additional reagents. If an association between a certain serotype and potential outbreak is suspected further testing is done (Scheutz et al., 2004).

Other techniques used in identification include: immunoblotting techniques; tissue culture cytotoxicity tests; enzyme-based immunoassays (ELISAs) which target the toxin (Weeratna and Doyle, 1991); immunomagnetic separation; lateral flow antigen detection; hybridization of DNA probes; DNA microarrays; PCR for additional virulence factor gene sequences (Paton and Paton, 2002); and typing using pulsed-field gel electrophoresis (PFGE) (Atalla et al., 2000; Paton et al., 1996). Although useful in detecting and characterizing VTEC each of these methods are generally time-consuming, highly technical, and costly (Beutin et al., 2002). This limits the speed, availability, ease of using such methods in regular detection, and limits detection to central laboratories. A simple, robust, selective, universal analytical method to detect all VTEC is 
needed (Bettelheim, 2007; Johnson et al., 2006) to prevent exposure, ensure proper clinical diagnosis (Couturier et al., 2011), and to prevent false attribution. Since all VTEC produce VT, a differential media indicating VT production would provide the simplest means of augmenting detection strategies through enhanced isolation.

Overproduction of VT induced by antibiotics can be used to aid in VT detection. Addition of mitomycin (a potent DNA crosslinker) can be used in growth media to upregulate toxin production and phage genes. This increase makes it easier to identify VTEC in samples using immunoblotting or other VT targeting detection techniques (Hull et al., 1993).

\subsection{Aptamers}

\subsubsection{General Information}

Aptamers are synthetically produced single-stranded oligonucleotides (DNA or RNA) which have a high binding affinity and selectivity for their target molecules (Famulok et al., 2000). Aptamers can be generated for almost any target through a process called Systematic Evvolution of Ligands by Exponential Enrichment (SELEX) first described by Ellington and Szostak (1990) and Tuerk and Gold (1990). SELEX is outlined in Figure 3 and reviewed by Stoltenburg et al. (2007). Briefly, a pool of nucleotide sequences is synthesized with a variable length of randomized nucleotides between two primer-binding regions. This pool can have as few as 15 random bases, but longer aptamers can produce a wider and more complex range of structures (Stoltenburg et al., 2007). The pool is subjected to cycles of positive selection for the target (and often a 
counter-selection against similar non-target species). The desired sequences are separated and amplified by PCR to create a pool of sequences for the next cycle. This will increase the amount of sequences that bind strongly (and selectively) to the target. After as many as 20 rounds the sequences which have the highest affinity for the target are isolated, sequenced, and further characterized (Stoltenburg et al., 2007). The affinity of an aptamer is determined by its three-dimensional shape and stable interaction of that complex structure with the target molecule (Hermann and Patel, 2000). Affinity is measured by the dissociation constant $\left(\mathrm{K}_{\mathrm{d}}\right)$, and typically ranges from picomolar to nanomolar levels for aptamers (Jayasena, 1999).

Aptamers can be developed with RNA though they tend to be less chemically stable and degrade more easily (Hamula et al., 2006). Aptamers can have augmented nuclease resistance by the incorporation of modified bases. This can be done prior to selection with a modified pool or after selection. Frequently the modified bases are 2' modified with fluorine or amino groups, as well as substituting sulfur for oxygen in the

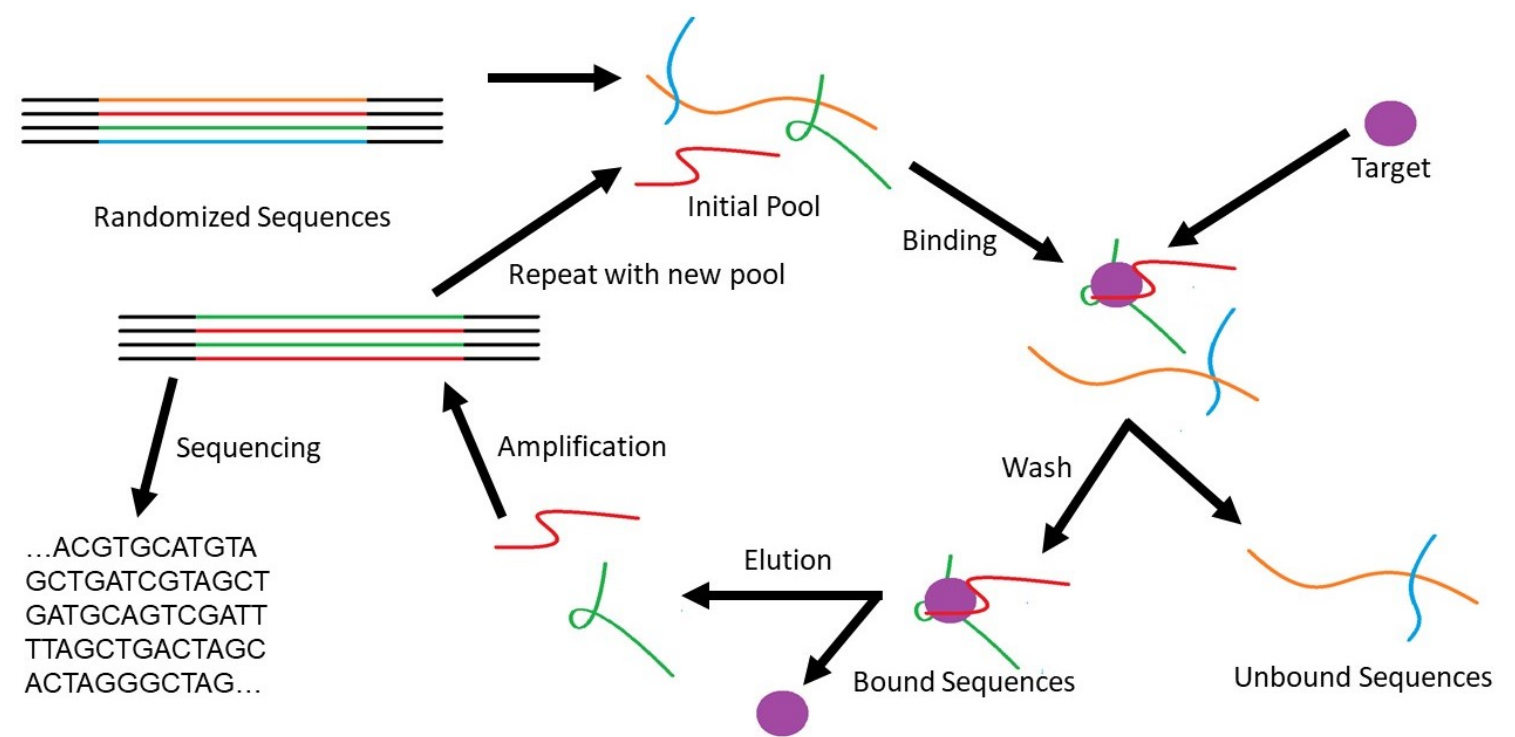

Figure 3 - Generalized overview of the SELEX process used to select aptamers for specific targets 
phosphate backbone among others (Pinheiro and Holliger, 2014). More recently 'xeno nucleic acids' (XNAs) which replace the sugar backbone have been used as they are poorly recognized by natural nucleases (Pinheiro and Holliger, 2014). Selection or mutation of polymerases has allowed XNAs to be efficiently synthesized (Pinheiro et al., 2012). These modifications have an influence on tertiary structure so modification of an existing aptamer is likely to result in reduced affinity (Ruckman et al., 1998). Exonuclease protection can be achieved by capping with small molecules not recognized by degradation enzymes (Jayasena, 1999). Aptamers selected from pools of peptides also exist although selection is a more complex process and the resulting peptides are also, by nature of their chemical structure, less stable than DNA aptamers.

Aptamers can be selected for a large variety of target molecules ranging from small molecules to antigens on whole cells, and as such, aptamers have a vast potential of possible applications including diagnostics and treatments similar to antibodies earning them the nickname chemical antibodies (Zimbres et al, 2013). Comparatively, aptamers have a number of advantages. One advantage is that they can match or surpass the discrimination between target and non-target molecules. For example, an RNA aptamer for theophylline was 10 times as efficient in distinguishing it from caffeine compared to antibodies (affinity 10,000 times stronger than for caffeine) (Jenison et al., 1994). DNA aptamers especially have greater chemical stability, are easily reproduced, produced at larger scales, and are readily modifiable than antibodies (Zimbres et al., 2013). Additionally, aptamers have the advantage of in vitro development, ability to be developed against native toxins, chemical synthesis, and reusability (reviewed in Bruno, 
2015). Aptamers also have the ability for target binding to be mediated by binding to a complement sequence. This can be used both as an antidote in therapeutically as well as in the design of target sensing schemes (Bompiani et al., 2012; Nutiu and Li, 2004). Aptamers can also be used in a chemically diverse environment and still selectively interact with their target (Hwang et al., 2016; Shi et al., 2011). This includes aptamer bacterial detection in diluted growth media (Dwivedi et al., 2013; Suh and Jaykus, 2013). Use of an aptamer for 0157 LPS in lateral flow assays allowed detection of E. coli 0157 at concentrations down to $10 \mathrm{CFU} / \mathrm{mL}$ based on aptamers targeting outer membrane proteins (Wu et al., 2015). Aptamers can also be used in 'western' blotting schemes for the detection of toxins or other proteins (Bruno and Sivils, 2016). It has been shown that aptamers selected for whole cells labeled with quantum dots are effective at detecting E. coli and Salmonella enterica in beef trim, baby spinach, and diced tomato enriched samples (Bruno et al., 2016).

\subsubsection{Aptamers Selected for VT}

Regarding aptamers selected for the detection of VTEC there are a handful which have been published. These are described briefly in Table 3. These sequences have been modified and used in combination with labeled complement sequences resulting in significant sensitivity. No single-strand, modified aptamer sensor system has been published.

Additionally, some other patents for aptamer sequences for VT have been filed. These patents are for: RNA or DNA aptamer sequences for VT1 as well as a SELEX 
Table 3 - Aptamer sequences selected for verotoxin

\begin{tabular}{|l|l|l|l|}
\hline Year & Aptamer seqs. & Description & Reference \\
\hline 2004 & 11 DNA seqs. for & Inhibit VT toxicity in HK-2 cell lines (100 pg/mL \\
VT1A and VT1B; & $\begin{array}{l}\text { VT, } 250 \mu \mathrm{g} / \mathrm{mL} \text { aptamers); Conjugated with } \\
\text { seqs. and } \\
\text { selection. }\end{array}$ & $\begin{array}{l}\text { quantum dots, as DNA capture elements was } \\
\text { shown to be as sensitive as FITC conjugated } \\
\text { antibodies }\end{array}$ & $\begin{array}{l}\text { Kiel } \\
\text { 2004a; Kiel } \text { et } \\
\text { al., 2004b; US } \\
\text { 2004/0023265 } \\
\text { A1 }\end{array}$ \\
\hline 2008 & $\begin{array}{l}\text { 1 DNA seq. for } \\
\text { Shiga toxin }\end{array}$ & $\begin{array}{l}\text { For use fluorescently labeled with a quencher } \\
\text { molecule labeled complement strand; no results } \\
\text { or characterization related to the seq. }\end{array}$ & $\begin{array}{l}\text { Fan } \text { et al., } \\
2008\end{array}$ \\
\hline 2014 & $\begin{array}{l}\text { 6 RNA seqs. for } \\
\text { VT2. }\end{array}$ & $\begin{array}{l}\text { 4 seqs. weakly inhibited VT2 binding to HeLa cells } \\
\text { (78-50\% inhibiting), none neutralizing toxicity } \\
\text { (max. 60\% cell survival) even at concs. nearly } \\
\text { 2000x to toxin; post-selection incorporation of 2' } \\
\text { fluorine caused loss of binding ability. }\end{array}$ & $\begin{array}{l}\text { Challa et al., } \\
2014\end{array}$ \\
\hline 2016 & DNA seq. for VT1 & $\begin{array}{l}\text { Labeled with quantum dots in an ELISA-like assay } \\
\text { down to a detection level of 0.1 ng/mL }\end{array}$ & $\begin{array}{l}\text { Bruno et al., } \\
2016\end{array}$ \\
\hline 2015 & $\begin{array}{l}\text { Peptide seq. for } \\
\text { VT }\end{array}$ & $\begin{array}{l}\text { Selectively decreases fluorescence in the } \\
\text { presence of VT. }\end{array}$ & $\begin{array}{l}\text { Manandhar } \text { et } \\
\text { al., 2015 }\end{array}$ \\
\hline
\end{tabular}

scheme for their selection patented in Japan in 2001 and later in the US in 2006 (No. US 2006/0008841 A1); one DNA aptamer sequence for VT2 and the method for use in ELISA-like detection with biconjugated quantum dots patented in the US in 2014 (No. US $8,790,877$ B2); and a DNA aptamer sequence for B subunits of VT2 which had some toxicity neutralization ability as well as the method of selection patented in Russia in 2015 (No. RU 2566552 C1).

\subsubsection{Aptamer Beacons}

Aptamers can be modified into aptamer beacons to combine the specific recognition abilities of the aptamer sequences with the ability to transduce signal analogous to that of molecular beacons. Molecular beacons are single-stranded DNA 
which has been modified to contain a fluorophore / quencher pair held together in a self-hybridizing hairpin (stem-loop) conformation (Tyagi et al., 1998). This formation keeps the fluorophore and quencher in close proximity quenching fluorescence. In the presence of a complementary DNA molecule the beacon will hybridize, changing conformation, separating the fluorophore and quencher, producing a measurable increase in fluorescence (Tyagi et al., 1998). The system can be modified to open in the presence of a non-DNA target by using an aptamer sequence since aptamer binding is often accompanied by a conformational change ((Shi et al., 2011; Song et al., 2008). This can be accomplished by addition of complementary bases, to form a hairpin structure, and a pair of fluorophore and quencher molecules (Nutiu and Li, 2004). The additional bases should be complementary to the aptamer bases important in target binding (Hamaguchi et al., 2001). The aptamer beacon should change conformation upon interact with the target resulting in an increase in fluorescence. The use of aptamer beacons to detect proteins has been previously described (Hamaguchi et al., 2001; Yamamoto and Kumar, 2000). Aptamer beacons have been successfully used with ions, small molecules, and proteins (reviewed in Wu et al., 2013).

Aptamer beacons can be selected for directly (Rajendran and Ellington, 2003), but often are prepared by modifying an existing aptamer sequence. Although electrochemical and colorimetric aptamer beacons are used regularly, primarily fluorescent labels are chosen due to the many benefits of fluorescent detection including: high sensitivity, non-invasive visual detection, multiplex detection, small dye size, ease of labeling, availability of fluorophores and quenchers, and real-time 
detection capability (Wu et al., 2013; Song et al., 2008). Aptamer beacons in a dual labeled hairpin format work via competitive interaction of the aptamer sequence with the complementary bases or the target molecule. These beacons can be designed to be an on-switch or off-switch mechanism, although an on-switch mechanism is typically preferable to increase sensitivity. However, DNA degradation of on-switch beacons can increase background, lower sensitivity, and cause false positives (Larkey et al., 2016; Shi et al., 2011). Generally, these aptamer beacons have only been used qualitatively or semi-quantitatively in assays (Song et al., 2008).

An aptamer beacon to detect VT offers potential as a simple method of detecting VTEC by application to a culture of enriched $E$. coli and visualizing with a standard UV lamp. This could be applied by screening enrichment broth samples for further isolation. More significantly, it could also be used to enhance isolation of VTEC colonies on a solid media in a similar way with fluorescent colonies. Furthermore, an aptamer beacon could be used to confirm VT production after isolation. If need be, a fluorescence spectrophotometer could be used to take sensitive readings on smaller samples.

\subsection{Thesis Objectives}

The objective of this study was to determine the potential of fluorescently labeled aptamer beacons in detection of VTEC. An aptamer previously selected for VT by Fan et al. (2008) by standard SELEX was modified to serve as proof of concept for the use of fluorescent aptamer beacons in detecting VT. The aptamer was also characterized as no information on selectivity or affinity strength had been included in the previous 
publication. To assist in future VT aptamer beacon use, comparison of fluorophorequencher pairs was performed to investigate optimal combinations with highest signal in relevant environments. Finally, a short fluorophore-quencher labeled DNA oligonucleotide was investigated as an enzymatic substrate for VT as an alternative differential reagent for detection. 


\subsection{Aptamer Beacon Proof of Concept}

\section{$2.1 \quad$ Introduction}

\subsubsection{The Benefit of a VT Aptamer Beacon}

Verotoxin (VT) is the definitive virulence factor produced by verotoxin-producing E. coli (VTEC) (Croxen et al., 2013). The isolation of VTEC strains can be challenging as their colonies are not morphologically distinct from other E. coli strains. However, VT production could be used to identify them (Atalla et al., 2000). Unfortunately, current methods for the identification of VTEC strains are highly technical, time-consuming, and expensive. A simpler, cheaper, VTEC differentiation method would be of great benefit for food safety analysis and clinical diagnosis.

Aptamers selected for VT offer potential for detection by modification into a structure-switching signalling aptamer, or aptamer beacon (Nutiu and Li, 2004). These aptamer beacons are formed by addition of a complementary region with a fluorophore and quencher molecule, normally held in close proximity. In the presence of the target molecule the aptamer beacon will change conformation to better interact with the target, increasing the distance between the fluorophore and the quencher, resulting in an increase in fluorescence. Aptamers can be easily modified, synthesised chemically, and stored for prolonged periods (Zimbres et al., 2013) making them cost effective and easy to work with. Aptamer beacons also offer the potential of sensitive, real-time detection (Wu et al., 2013) making it a time efficient method as well.

Ideally, the application of a VT aptamer would be in the isolation phase of VTEC detection. This could either be directly in media before inoculation with bacteria, or 
potentially as an application to colonies on media following growth. Tryptone soya agar (TSA) is a general nonselective media used commonly as a microbial growth media, and a number of other common media share primary components with TSA. An aptamer beacon that could function in these conditions would be ideal for augmenting and simplifying analytical detection of VTEC.

\subsubsection{Fluorescence Measurement}

Fluorescent labels are commonly used in detection sensors. Some reasons for this include their sensitivity at low concentrations, small size of fluorescent dyes, and ease of labeling (Wu et al., 2013). Fluorescent substances absorb light of certain wavelengths, causing electronic excitation. This additional energy is released through an emission of light as the electron relaxes to a lower energetic state.

Fluorimetry measures the intensity of that emission. A specific wavelength or range of wavelengths is selected from a light source by a monochromator or an optical filter. This selected light is shone into samples. Any resulting fluorescence from the samples is emitted and can be detected. Typically, this emission is measured at a $90^{\circ}$ angle from the source to minimize detection of excitation light (resulting in background

noise). The emission light can then pass through similar selection and to a light detector.

The sensitivity of fluorescence is due to the relative signal observed between absence and presence of analyte. If the background noise is kept low, the increase even with very low concentrations of analyte is very significant. Colorimetric absorption spectroscopy however measures only small changes in the initial and final intensity of 
light from the source caused by absorption by the analyte. As such there is always some background signal.

If fluorescent samples are too concentrated, however, scattering and absorption can occur within the sample lowering the intensity of the emitted light (Henderson, 1977). At lower concentrations the fluorescence intensity $(F)$ is proportional to the incident light intensity $\left(I_{o}\right)$ and analyte concentration $(c)$, where $k$ is a constant that depends on the fluorophore:

$$
F=k I_{o} c
$$

In these experiments however, monochromatic filters are used for selection of excitation light. They allow a fixed and fairly wide band of wavelengths through, simplifying experiments and allowing more light through for higher sensitivity. The excitation and emission wavelengths and bandwidths should be chosen carefully according to the fluorophore's spectra to maximize sensitivity. Additionally, samples in these experiments were excited from the same direction that emission light is detected using mirrors. Dichroic mirrors have special coatings that allow certain wavelengths to transmit and others to reflect. As such, excitation and emission wavelengths can be separated if the dichroic cut-off wavelength is suitable for the studied fluorophore.

In addition to fluorescent emission, an excited fluorophore can also lose energy as heat, or transferring energy to another molecule in close proximity. The transfer of energy that results in a loss of fluorescence signal is referred to as quenching and has been exploited for a number of purposes. These include nanoscale distance measurements, protein interactions, in vivo labelling and more (Selvin, 2000). The two 
main types of quenching are contact quenching and fluorescence resonance energy transfer (FRET) (Marras et al., 2002). FRET quenching occurs when energy is transferred to an appropriate acceptor molecule within 20-100 $\AA$. This reduces the donor's fluorescence intensity and excited state lifetime. The acceptor can be any quencher or fluorophore molecule with good absorption spectra overlap with the donor's emission spectrum (Marras et al., 2002). FRET quenching efficiency is highly dependent on the distance between the fluorophore and quencher (by a $1 / \mathrm{r}^{6}$ distance dependence) and their relative orientations (Marras et al., 2002). Contact quenching occurs when the fluorophore and quencher are close enough to form a strongly quenched complex that turns most of the excitation energy directly into heat (Marras et al., 2002).

\subsection{Chapter Objectives}

The aptamer sequence published by Fan et al. (2008) was modified into an aptamer beacon and synthesized. The beacon was first characterized using a complement DNA sequence and experimental conditions optimized for general use. The aptamer beacon was tested with commercial verotoxin to observe if the aptamer had an affinity for the toxin. Finally, the beacon system was tested on a solid agar media with complement to observe if there is a visible increase in fluorescence.

Figure 4 shows a schematic for the beacon's increased signal in the presence of complement or toxin. If there is an interaction, the beacon should change conformation, producing more fluorescence intensity. 


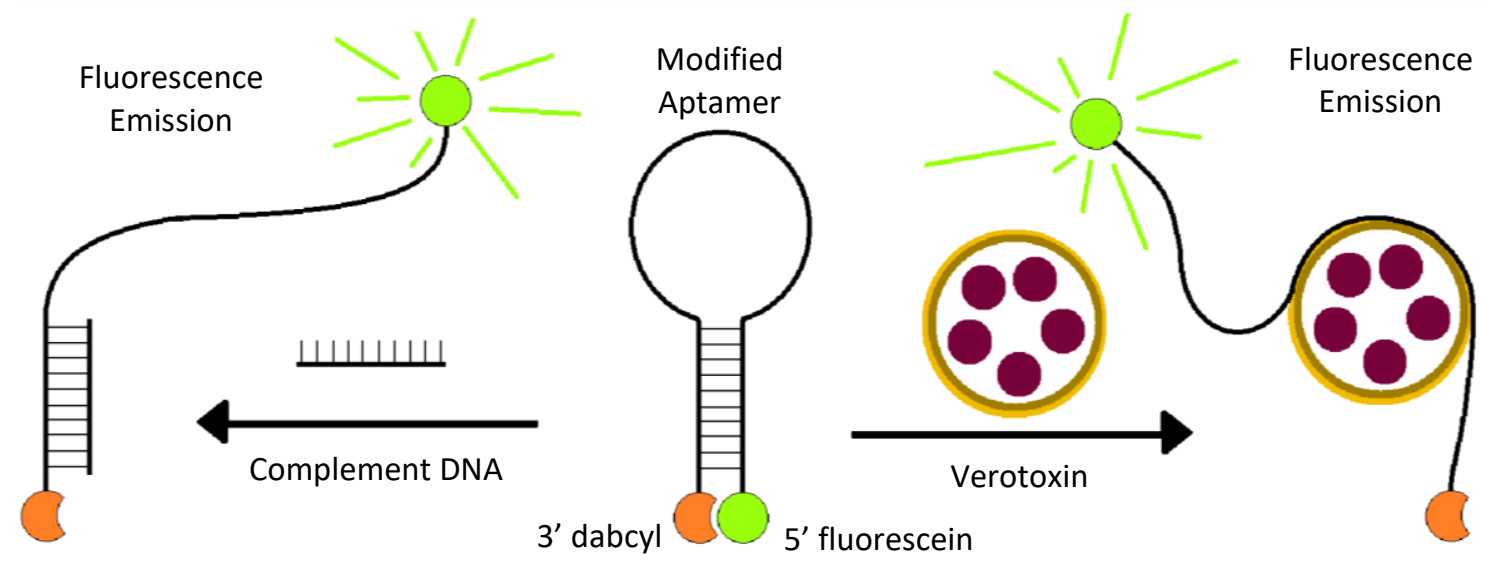

Figure 4 -Schematic diagram of the modified aptamer beacon with no fluorescence (middle) and interaction with complement DNA (left) and verotoxin (right) resulting in a conformational shift, producing an increased fluorescent emission.

\subsection{Statement of Contributions}

Fluorescent control DNA used in the following experiments was prepared by Nadine Frost. Mass spectrometric analysis was performed by Alexander Wahba, McGill University, Mass Spectrometry Facility.

\subsection{Materials and Methods}

\subsubsection{Buffers}

Table 4 - Buffers used in Chapter 2.0 Aptamer Beacon Proof of Concept

\begin{tabular}{|l|l|}
\hline Name & Components \\
\hline TEB (tris-EDTA Buffer) & $\begin{array}{c}10 \mathrm{mM} \text { tris, } 1 \mathrm{mM} \text { EDTA, } 10 \mathrm{mM} \mathrm{NaCl}, 10 \mathrm{mM} \mathrm{KCl} \\
\mathrm{pH} 7.4 \text { (adjusted with } \mathrm{HCl} \text { and NaOH) }\end{array}$ \\
\hline TES (tris-EDTA high salt) & $\begin{array}{c}10 \mathrm{mM} \text { tris, } 0.5 \mathrm{mM} \text { EDTA, } 0.5 \mathrm{M} \mathrm{NaCl}, 0.5 \mathrm{M} \mathrm{KCl} \\
\mathrm{pH} 7.4 \text { (adjusted with } \mathrm{HCl} \text { and NaOH) }\end{array}$ \\
\hline 5x TBE (tris, Boric Acid, EDTA) (4L) & $0.5 \mathrm{M}$ tris, 0.5 M boric acid, 0.01 M EDTA \\
\hline
\end{tabular}

2.4.2 Preliminary structural analysis and aptamer beacon design

Predicted secondary structures were determined using RNA Structure and Mfold software (Reuter and Matthews, 2010; Zuker, 2003). 


\subsubsection{DNA Synthesis, purification, and quantification}

\subsubsection{DNA Synthesis}

DNA was synthesized using a MerMade 6 system (BioAutomation Corp.). 3' modified CPG columns with dabcyl (4-[4'-dimethylaminophenylazo]benzoic acid) (Figure 5), and 5'-fluorescein modified phosphoramidite (Figure 6) were used to prepare the two aptamer beacons, while normal columns were used for the unlabeled complement. Reagents were purchased from GlenResearch (VA, USA). DNA sequences synthesized were as follows, the underlined sections are complementary to form a hairpin: C10: 5'-G CTA GTT ACC-3'

A10: 5'-Fluorescein-GGT AAC TAG CAT TCA TTT CCC ACA CCC GTC CCG TCC AT ATG CTA GTT ACC-Dabcyl-3'

\section{A15: 5'-Fluorescein-GGT AAC TAG CAT TCA TTT CCC ACA CCC GTC CCG TCC AT AT TGA ATG CTA GTT ACC-Dabcyl-3'}

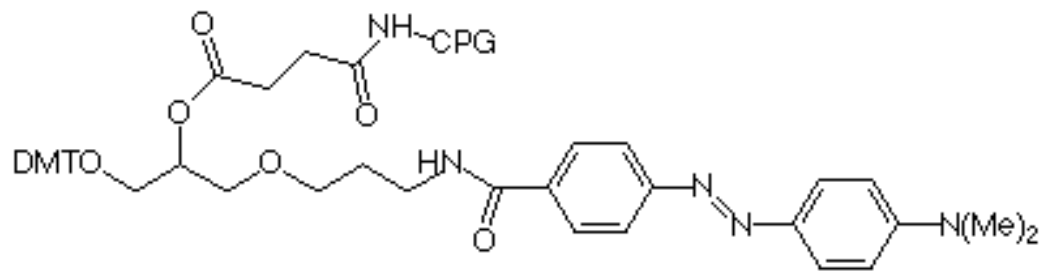

Figure 5 - Dabcyl CPG (1-Dimethoxytrityloxy-3-[O-(N-4'-carboxy-4-(dimethylamino)azobenzene)-3-aminopropyl)]-propyl-2-O-succinoyl-long chain alkylamino-CPG)

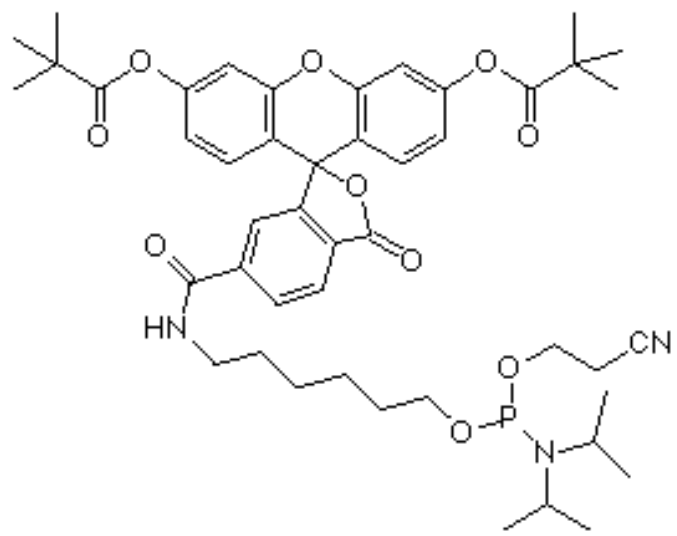

Figure 6-5' fluorescein phosphoramidite (6-(3',6'-dipivaloylfluoresceinyl-6-carboxamido)-hexyl1-O-(2-cyanoethyl)-(N,N-diisopropyl)-phosphoramidite) 


\subsubsection{Sample Clean-up and Quantification}

The synthesized DNA was cleaved from the beads by incubation in $28 \% \mathrm{NH}_{4} \mathrm{OH}$ for three hours at $55^{\circ} \mathrm{C}$ and overnight at room temperature. The DNA was separated by centrifugation (5000 x g, $3 \mathrm{~min}$ ). DNA samples were purified by polyacrylamide/urea gel electrophoresis (12\% or $19 \%$ gels). Gels were run at constant amperage and imaged under UV and fluorescent lights using Alphalmager (Alphalnnotech). DNA was extracted by gels being gently crushed, and incubated with shaking in water at $37^{\circ} \mathrm{C}$ overnight and desalted. DNA was quantified using a Cary 300 Bio UV-Visible Spectrophotometer (Varian) at $260 \mathrm{~nm}$. Samples were dried and stored in a $-20^{\circ} \mathrm{C}$ freezer.

\subsubsection{Mass Spectrometric Analysis}

Samples were sent for mass spectrometric analysis and analyzed by LC-MS using a Dionex Ultimate 3000 UHPLC coupled to a Bruker Maxis Impact QTOF mass spectrometer in negative ESI mode. Samples were run through an Acclaim RSLC 120 C18 column $(2.2 \mu \mathrm{M} 120 \mathrm{~A} 2.1 \times 50 \mathrm{~mm})$ using a gradient of $98 \%$ mobile phase $\mathrm{A}$ (100 mM HFIP and 5 mM TEA in $\mathrm{H}_{2} \mathrm{O}$ ) and $2 \%$ mobile phase $\mathrm{B}(\mathrm{MeOH})$ to $40 \%$ mobile phase $A$ and $60 \%$ mobile phase B in 8 minutes. The data was processed and deconvoluted using Bruker DataAnalysis software version 4.1.

\subsubsection{Other DNA}

A 95-base fluorescein labeled DNA oligonucleotide was used as a fluorescent control (FC). A 15-base DNA complement was ordered from Integrated DNA Technologies (IA, USA) with standard purification and desalting. 


\subsubsection{Fluorescence Measurements}

Samples were heated at $90{ }^{\circ} \mathrm{C}$ for 10 minutes and allowed to cool at room temperature for 30 minutes before use. This is to dissociate any potential intermolecular structures formed in solution prior to experimentation. Aptamer beacon was pipetted into buffer solutions with varying ratios of complement DNA. Solutions were given gentle agitation and promptly read by the fluorescence reader. Unless otherwise stated, fluorescence readings were taken at room temperature. FC was used as a fluorescent control at the same concentrations in each test.

Testing was done in $200 \mu \mathrm{L}$ of solution in black 96-well plates (Nunc; Thermo Scientific) and read with use of a Synergy 2 multi-mode reader (BioTek). These experiments were done with varying conditions (concentration, buffer, beacon: complement ratio, complement, temperature, read times, preparation), but followed the basic method above. Excitation was done at $495 \pm 10 \mathrm{~nm}$, emission read at $528 \pm 20$ $\mathrm{nm}$, with a dichroic 'top 510' mirror, with a sensitivity level of 90. Data was collected using Gen5 software (BioTek).

Testing with VT1a and VT2a were done following the same method, but replacing complement with VT (List Biological Labs, Inc.; CA, USA).

\subsubsection{Linear Fluorescence Range}

Solutions of FC were prepared with concentrations of $2.5 \times 10^{-3}$ to $5 \mu \mathrm{M}$. The samples were plated and read to determine the linear fluorescent range. 


\subsubsection{Effect of Temperature and Melting Temperature Determination}

Solutions of $\mathrm{A} 10$ and $\mathrm{FC}$ were prepared to $1 \mu \mathrm{M}, 75 \mathrm{nM}$, and $5.7 \mathrm{nM}$. Samples

were plated. Readings were taken every minute for 3 minutes at room temperature, and every minute for 3 minutes at $60^{\circ} \mathrm{C}$ (heating took about 40 minutes).

Melting temperature determination for aptamer beacons $\mathrm{A} 10$ and $\mathrm{A} 15$ was done using $3 \mathrm{~mL}$ of $2 \mu \mathrm{M}$ solutions in a Cary 300 Bio UV-Visible Spectrophotometer (Varian) with readings at $260 \mathrm{~nm}$. The solutions were initially heated to $80^{\circ} \mathrm{C}$. The solutions were then cooled to $20{ }^{\circ} \mathrm{C}$, heated to $80^{\circ} \mathrm{C}$, and finally cooled to $20^{\circ} \mathrm{C}$ at a rate of $0.5^{\circ} \mathrm{C} / \mathrm{min}$ with a reading taken every $1{ }^{\circ} \mathrm{C}$ producing three curves. The results were analyzed using SigmaPlot Standard Curves Analysis software.

\subsubsection{Effect of Reannealing Media}

Solutions of $\mathrm{A} 10$ and $\mathrm{FC}$ were heated to $90{ }^{\circ} \mathrm{C}$ for 10 minutes and allowed to cool at room temperature for 30 minutes. Solutions were either $50 \mu \mathrm{M}$ in water or $5 \mu \mathrm{M}$ in TEB. Solutions were diluted to $1 \mu \mathrm{M}, 100 \mathrm{nM}$, and $10 \mathrm{nM}$. The samples were plated and readings were taken every minute for 10 minutes at room temperature.

Similarly, solutions of $\mathrm{A} 10$ were prepared and mixed with $\mathrm{C} 10$ to a 1:4 ratio for final $\mathrm{A} 10$ concentrations of $1 \mu \mathrm{M}, 100 \mathrm{nM}$, and $10 \mathrm{nM}$ and $\mathrm{C} 10$ concentrations of $4 \mu \mathrm{M}$, $400 \mathrm{nM}$, and $40 \mathrm{nM}$.

\subsubsection{Effect of Buffer Salinity}

Solutions of $\mathrm{A} 10$ and $\mathrm{FC}$ were heated to $90{ }^{\circ} \mathrm{C}$ for 10 minutes and allowed to cool at room temperature for 30 minutes. Solutions were either $50 \mu \mathrm{M}$ in water or $5 \mu \mathrm{M}$ in 
TEB or TES. Solutions were diluted to $1 \mu \mathrm{M}, 100 \mathrm{nM}$, and $10 \mathrm{nM}$ in TEB or TES. The samples were plated and readings were taken every minute for 10 minutes at room temperature.

Similarly, solutions of A10 were prepared in TES or TEB and mixed with C10 in ratios of $1: 0,1: 1,1: 2$ and $1: 4$ for final $A 10$ concentrations of $1 \mu \mathrm{M}, 100 \mathrm{nM}$, and $10 \mathrm{nM}$ with corresponding $\mathrm{C} 10$ concentrations. The samples were plated and readings were taken every two minutes for 60 minutes at room temperature.

\subsubsection{Effect of Complement Length}

Solutions of $\mathrm{A} 10$ and $\mathrm{FC}$ in TEB or TES were diluted and mixed with $\mathrm{C} 10$ or C15 at a 1:1 ratio for final $A 10$ concentrations of $1 \mu \mathrm{M}, 100 \mathrm{nM}$, and $50 \mathrm{nM}$. The samples were plated and readings were taken every two minutes for 60 minutes at room temperature.

\subsubsection{Effect of Toxin on Probe Fluorescence}

Solutions of A10, and FC in TES were prepared. A10 was mixed separately with C15, VT1a, or VT2a. Solutions had final concentrations of $50 \mathrm{nM}$ and 1:1 ratios of beacon to complement or toxin. Samples were plated in duplicate and replicated on a separate plate. This was also performed with A15.

\subsubsection{Visibility of Fluorescein on TSA}

Spotting of reagents was done on Tryptone Soya Agar (TSA). $10 \mu \mathrm{L}$ of FC were spotted onto a TSA plate at $50,5,0.5$, and $0.05 \mu \mathrm{M}$. On a second agar plate $10 \mu \mathrm{L}$ of FC were spotted at 50,40,30, 20, and $10 \mu \mathrm{M}$. After spotting, the agar plates were allowed to dry. Agar plates were visualized using a handheld UV lamp. Observations were 
recorded and pictures were taken. Fluorescence was easier to discern in person than afterwards with the images.

On separate plates testing with $\mathrm{A} 10$ and $\mathrm{C} 15$ were done. A10 was heated as normal. Subsequently, $10 \mu \mathrm{L}$ of $\mathrm{A} 10(50 \mu \mathrm{M})$ were spotted, as well as a $30 \mu \mathrm{M}$ FC positive control. After the plate had dried $10 \mu \mathrm{L}$ of $\mathrm{C} 15(100 \mu \mathrm{M})$ were spotted onto half of the A10 spots and allowed to dry. A separate plate was done reversing the order of reagent application. Plates were visualized using a handheld UV lamp. Observations were recorded and pictures were taken.

\subsubsection{Data Analysis}

Basic analysis of averages and standard deviations was done with Microsoft Excel software. All error bars shown on graphs represent standard deviation. Preliminary statistical analysis including one-way ANOVA, post-hoc Tukey and homogeneity of variance tests, was done with SPSS Statistics software version 22 (IBM) at a significance level of $p=0.05$.

\section{$2.5 \quad$ Results}

\subsubsection{Aptamer Beacon Design}

The A10 and A15 aptamer beacons were designed by using the 40-base randomized region of the forward (+) aptamer sequence published by Fan et al. (2008) and adding complementary bases to the $3^{\prime}$ end, as well as the fluorescent molecule fluorescein at the $5^{\prime}$ end and the quenching molecule dabcyl (4-[4'- 
dimethylaminophenylazo]benzoic acid) at the $3^{\prime}$ end.

Secondary structures were for the 40 -base aptamer sequence predicted using RNAstructure and Mfold online software (Reuter and Matthews, 2010; Zuker, 2003), both produced similar results (Figure 7A and Figure A-1). Minimal secondary structure was observed in each predicted structure.

Ten complementary bases were added for A10; and fifteen bases for A15. The analysis predicted the formation of hairpins composed of 12 and 15 pairing bases for $A 10$ and $A 15$, respectively (Figure $7 B$ and $C$ and Figure $A-2$ ).

A

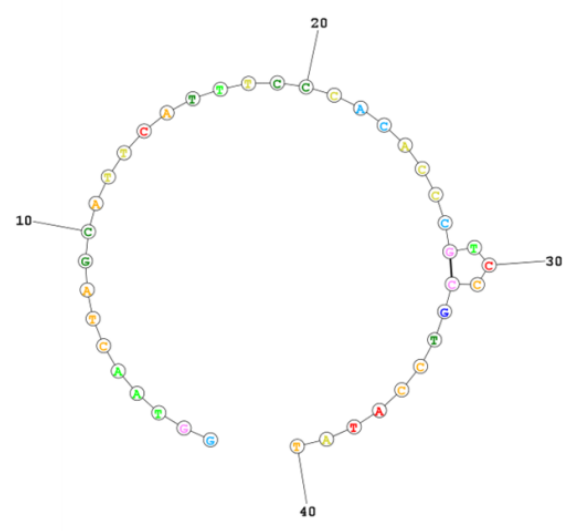

B

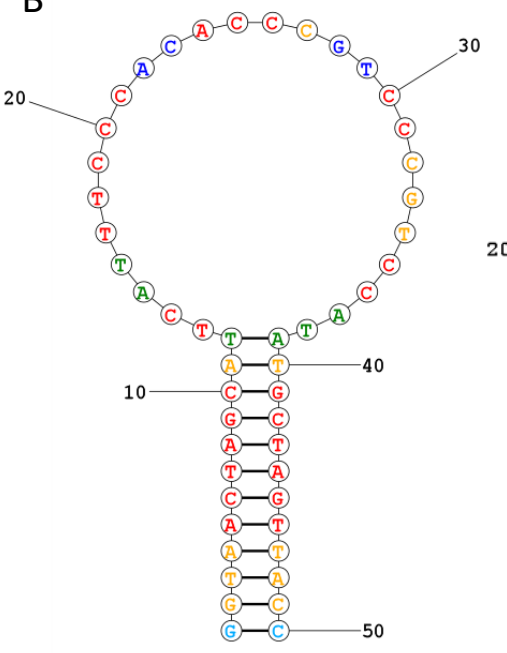

C

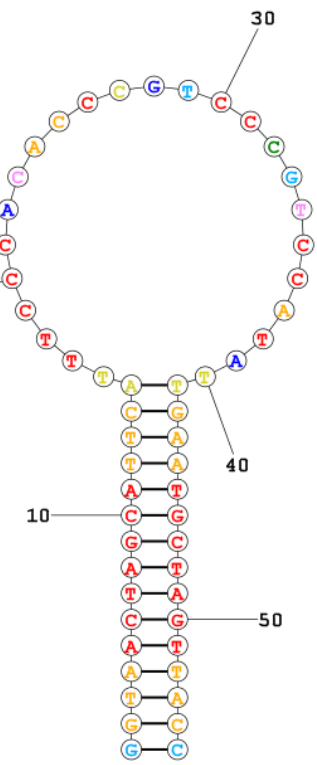

Figure 7-Secondary structure of Fan et al.'s 40 base forward (+) verotoxin DNA aptamer sequence predicted with RNAstructure software A) unmodified; B) with 10 complementary bases at the 3' end (A10); and C) with 15 complementary bases (A15) (Reuter and Matthews, 2010).

A complementary DNA sequence to be used as a positive control with A10 and A15 was also designed. A complement 10 bases long (C10) was predicted to have no secondary structure with both RNAstructure and Mfold software. Secondary structure was predicted for 11-20 bases. The predicted structures for a DNA complement 
composed of 15 bases had small hairpin formation (Figure 8). Similar structures for 1620 bases were predicted (not shown).
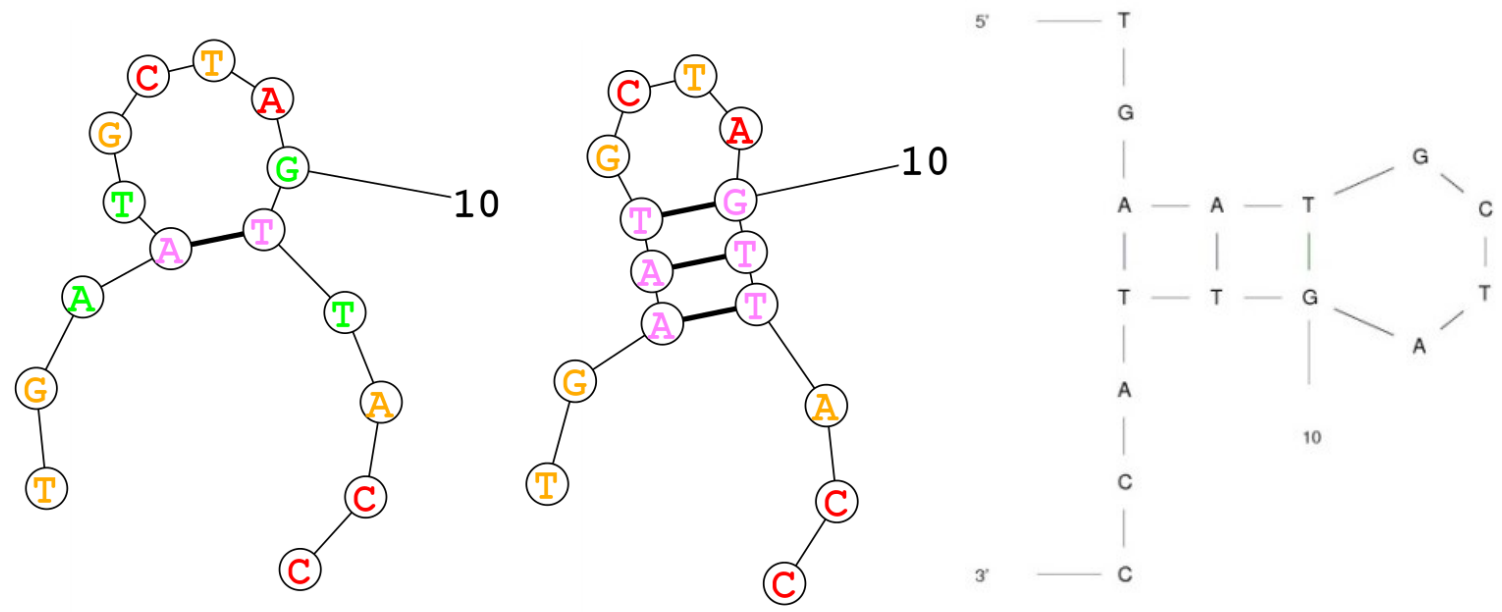

Figure 8- Secondary structure for a 15-base DNA complement (5' - TGA ATG CTA GTT ACC - 3') using RNAstructure (left) and Mfold (right) software (Reuter and Matthews, 2010; Zuker, 2003).

\subsubsection{Initial Fluorescence Testing with C10}

DNA samples were synthesized and purified. Acceptable purity was confirmed by mass spectrometric analysis (Appendix B samples 2B, 4B, and 6B). Upon confirmation of purity, the linear fluorescent range was determined (Figure A-3). A10 and A15 (1 $\mu \mathrm{M})$ were prepared in TEB buffer with C10 at beacon: complement ratios of 1:0, 1:1, 1:2, and 1:4. An increase in fluorescence was seen over an hour in each case with addition of C10, however, very high background fluorescence was observed for the beacon alone (Figure 9). A10 showed much higher fluorescence than A15. FC resulted in about $8.5 \mathrm{x}$ $10^{5}$ relative fluorescence units (RFUs), which was about $9 \mathrm{X}$ stronger than the beacons' resulting emission (not shown). 


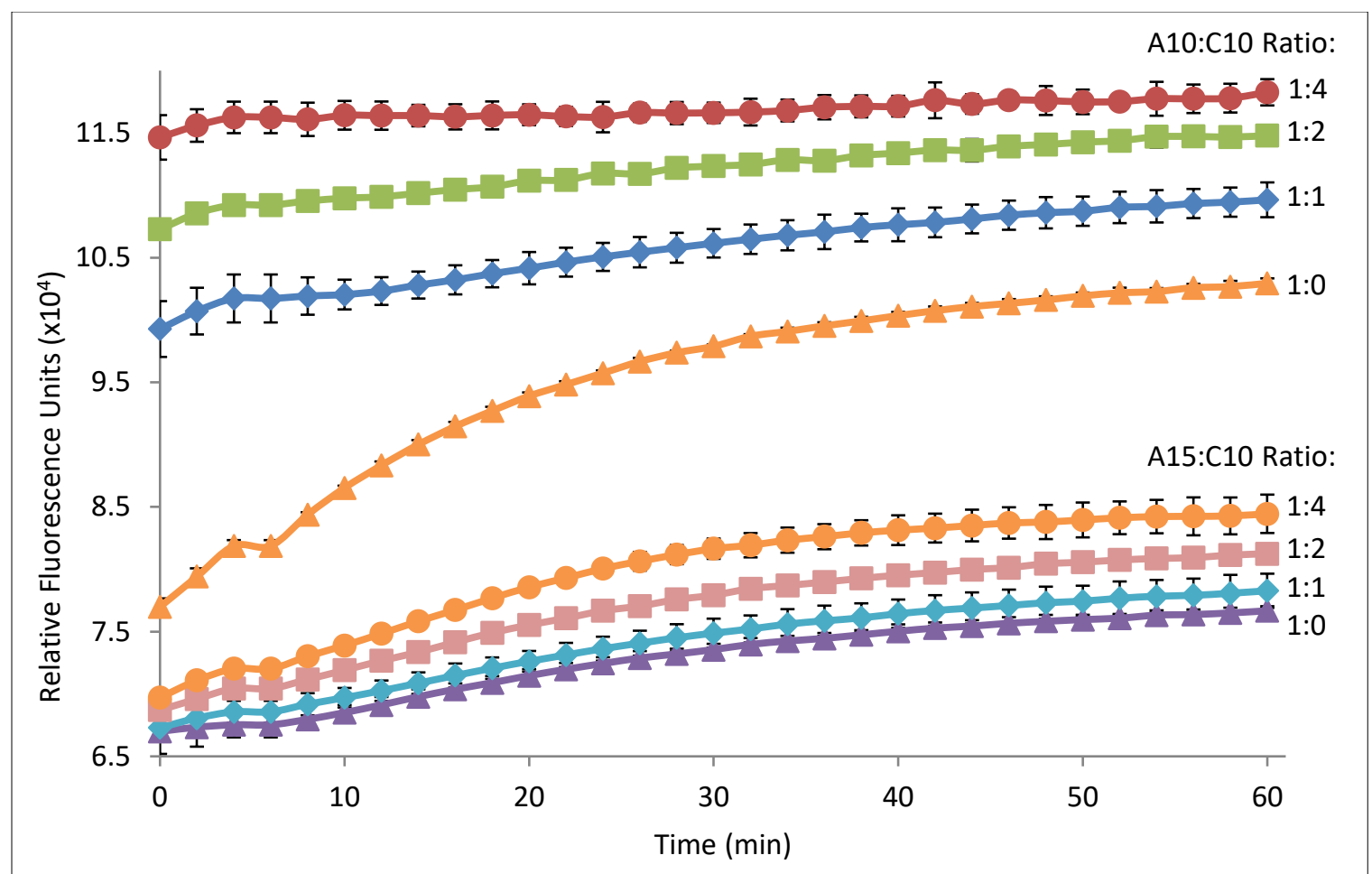

Figure 9 - Fluorescence intensity for fluorescein / dabcyl labeled aptamer beacon with 10 complementary bases (A10) and 15 complementary bases (A15) at $1 \mu \mathrm{M}$ with increasing of beacon: complement ratios (1:0, 1:1, 1:2, and 1:4) over an hour in TEB buffer. DNA complement was 10 bases long (C10). Fluorescent control values were about $85 \times 10^{4}$ RFUs. Data points represent the average of two wells (error bars show SD). Ex. $495 \pm 10 \mathrm{~nm}, \mathrm{Em} .528 \pm 20 \mathrm{~nm}$.

\subsubsection{Melting Temperature Determination and Effect of Temperature}

To discern the effect of experimental temperature on the system and if it could be optimized in any way the melting temperature of the aptamer beacons was determined (Figure 10). The melting temperatures for A10 and A15 were determined to be $42.2 \pm 0.6^{\circ} \mathrm{C}$ and $44.8 \pm 0.5^{\circ} \mathrm{C}$, respectively.

An experiment was conducted to observe the effects of temperature on the fluorescent emission. FC and A10 were prepared in TEB at a concentration of $1 \mu \mathrm{M}$ and readings were taken every minute for 3 minutes at room temperature, then again, every minute for 3 minutes after heating to $60{ }^{\circ} \mathrm{C}$ (Figure 11). Similar results were observed at 
$75 \mathrm{nM}$ and $5.7 \mathrm{nM}$ (Figure A-4 and Figure A-5). The FC signal decreased about 30\%, while A10's increased to about $190 \%$ compared to the values at room temperature.
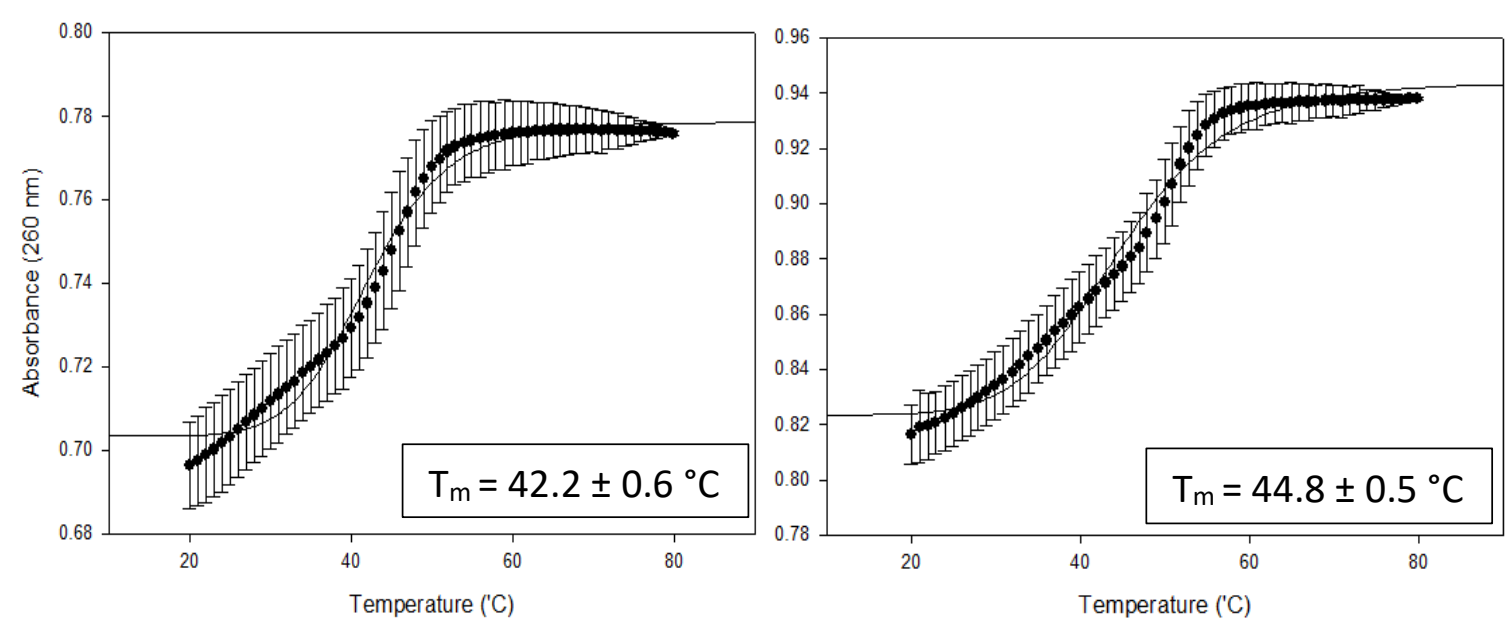

Figure 10 - Melting temperature curves for aptamer beacons with 10 complementary bases (A10) (left) and 15 complementary bases (A15) (right) prepared using $260 \mathrm{~nm}$ spectrophotometer absorbance readings and SigmaPlot software.

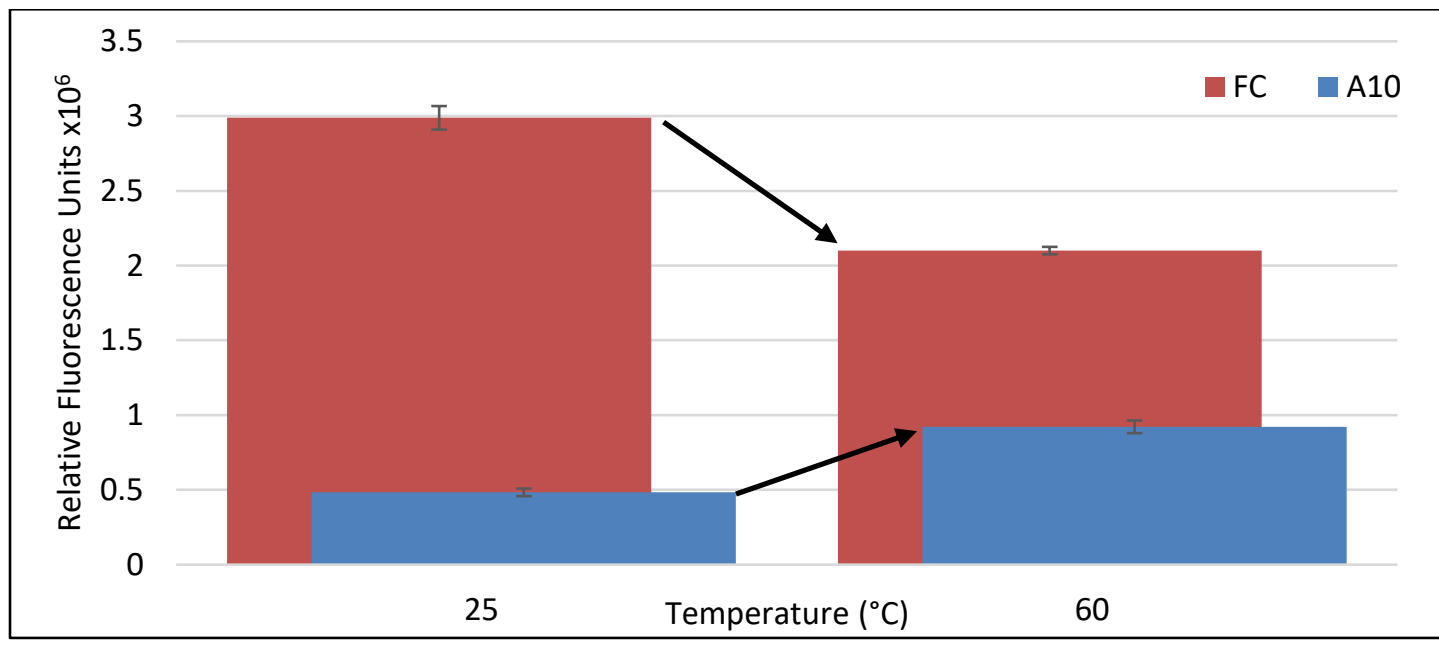

Figure 11 - Fluorescence intensity of fluorescent control DNA (FC) and fluorescein / dabcyl labeled aptamer beacon with 10 complementary bases (A10) at $1 \mu \mathrm{M}$ in TEB buffer. About 40 minutes passed while heating to $60^{\circ} \mathrm{C}$. Data points represent the average of 3 (FC) or 8 wells (A10) with error bars showing SD. Ex. $495 \pm 10 \mathrm{~nm}, \mathrm{Em} .528 \pm 20 \mathrm{~nm}$.

\subsubsection{Effect of Reannealing Media}

The effect of refolding conditions on resulting fluorescence was investigated. For each experiment aptamer beacons were denatured at $90^{\circ} \mathrm{C}$ and allowed to refold at 
room temperature for 30 minutes. $\mathrm{A} 10$ was heated at $50 \mu \mathrm{M}$ in water or $5 \mu \mathrm{M}$ in TEB before diluting. Samples were prepared with or without C10 (ratio 1:4). Readings were taken every minute for 10 minutes at room temperature at $1 \mu \mathrm{M}$ (Figure 12), $100 \mathrm{nM}$ (Figure A-6) and $10 \mathrm{nM}$ (Figure A-7). The difference in fluorescence between heating in buffer or water varied and showed an increase of $0-8 \%$ for the background fluorescence. This affected both resulting emission in the presence of $\mathrm{C} 10$ and background fluorescence.

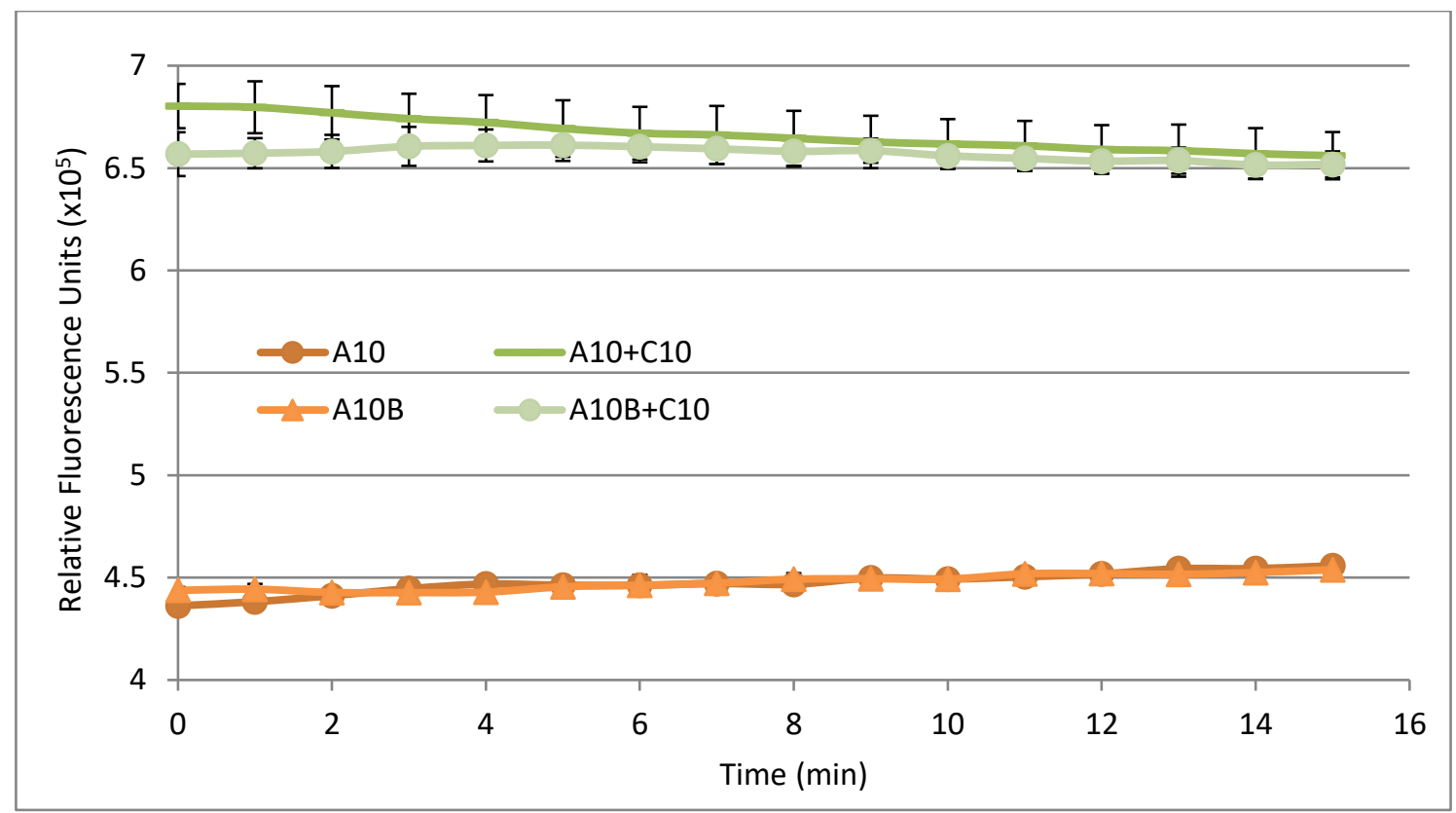

Figure 12 - Fluorescence intensity effects of refolding in TEB buffer (B) of fluorescein / dabcyl labeled aptamer beacon with 10 complementary bases (A10) $(1 \mu \mathrm{M})$ alone and in the presence of C10 (4 $\mu \mathrm{M})$. fluorescent control DNA (FC) had a value of about $30 \times 10^{5}$ RFUs. Data points are the average of 3 samples. Error bars show SD. Ex. $495 \pm 10 \mathrm{~nm}, \mathrm{Em} .528 \pm 20 \mathrm{~nm}$.

\subsubsection{Effect of Buffer Salinity}

The effect of buffer salinity on fluorescence was investigated. To further observe the effects of refolding in buffer on fluorescence A10 was heated in water or in buffer, both in TEB buffer (20 mM NaCl / KCl) or TES buffer (1 M NaCl / KCl). As with TEB, 
refolding in buffer resulted in a variable decrease of fluorescence and sometimes a small increase at $1 \mu \mathrm{M}$ (Figure A-8), $100 \mathrm{nM}$ (Figure A-9), and $10 \mathrm{nM}$ (Figure A-10).

To determine the effect of the buffer salinity upon the resulting emission, A10 was refolded in buffer, and mixed with C10 resulting in an A10 concentration of $1 \mu \mathrm{M}$ with A10:C10 ratios of 1:0, 1:1, 1:2, and 1:4 (Figure A-11). The TES sample fluorescence values were each about $45 \%$ less than the TEB samples, both background and resulting emission. Each C10 sample had higher fluorescence with 1:4 ratio having the largest. Notably, over the 60-minute period the background fluorescence for TES was fairly stable, while the resulting emission increased (Figure 13).

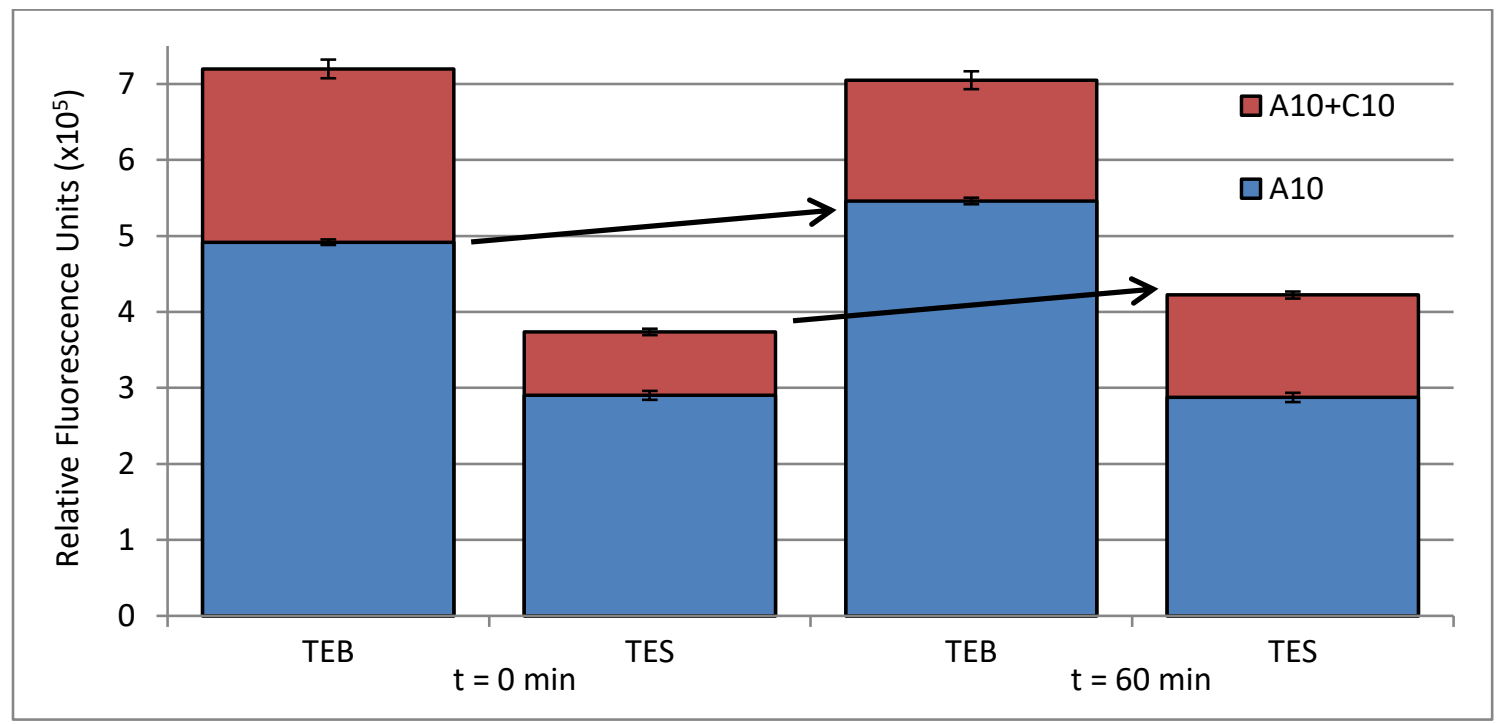

Figure 13 - Comparison of fluorescence intensity of fluorescein / dabcyl labeled aptamer beacon with 10 complementary bases $(A 10)(1 \mu M)$ in low or high salt buffer with or without $C 10(4 \mu M)$. Buffer had $20 \mathrm{mM}$ (TEB) or $1 M$ (TES) of NaCl/KCl. Data points are the average of 4 wells. Error bars show SD. Fluorescent control values were about $34 \times 10^{5}$ RFUs. Ex. $495 \pm 10 \mathrm{~nm}, \mathrm{Em} .528 \pm$ $20 \mathrm{~nm}$.

\subsubsection{Effect of Complement Length}

The length of complement DNA on fluorescence signal was investigated. A 15base complement ( $\mathrm{C} 15)$ was tested in comparison to $\mathrm{C} 10$ and run at a 1:1 ratio with $\mathrm{A} 10$. 
C15 was predicted to have minimal secondary structure (Figure 8). Samples of A10 were prepared in TEB or TES and mixed with C10 or C15 for final concentrations of $1 \mu \mathrm{M}$ (Figure 14). The 45\% decrease in TES was seen again for the background and C10 resulting signal; however, the fluorescence signal caused by C15 was not decreased and after about 30 minutes was equal to the values seen in TEB. In both cases C10 increased the fluorescence intensity by about $20 \%$ compared to the background. Again, the background in TEB increased while TES did not. Relatively, the resulting fluorescence was about $1.54 \mathrm{x}$ and $3.73 \mathrm{x}$ more than the background for TEB and TES, respectively. When repeated at $50 \mathrm{nM}$ similar results were observed (Figure 15). However, TES at all time points had a lower RFU value and interaction between $\mathrm{A} 10$ and $\mathrm{C} 15$ did not result in the same RFU as in TEB. The resulting fluorescence was about 1.13x and 1.61x greater than the background for TEB and TES, respectively. At $100 \mathrm{nM}$, slightly larger differences were found (Figure A-12).

\subsubsection{Effect of Concentration}

Finally, concentration of the beacon / complement was investigated. In each of the previous optimizations, testing was performed at concentrations around $1 \mu \mathrm{M}, 100$ $\mathrm{nM}$, and $10 \mathrm{nM}$. Each factor investigated was similar at lower concentrations except for C15 which caused the largest signal at higher concentrations.

\subsubsection{Effect of Toxin on Probe Fluorescence}

After having identified optimal conditions, the effect of verotoxin $1 \mathrm{a}$ and $2 \mathrm{a}$ on fluorescence of the aptamer beacons were investigated. Samples were plated in 


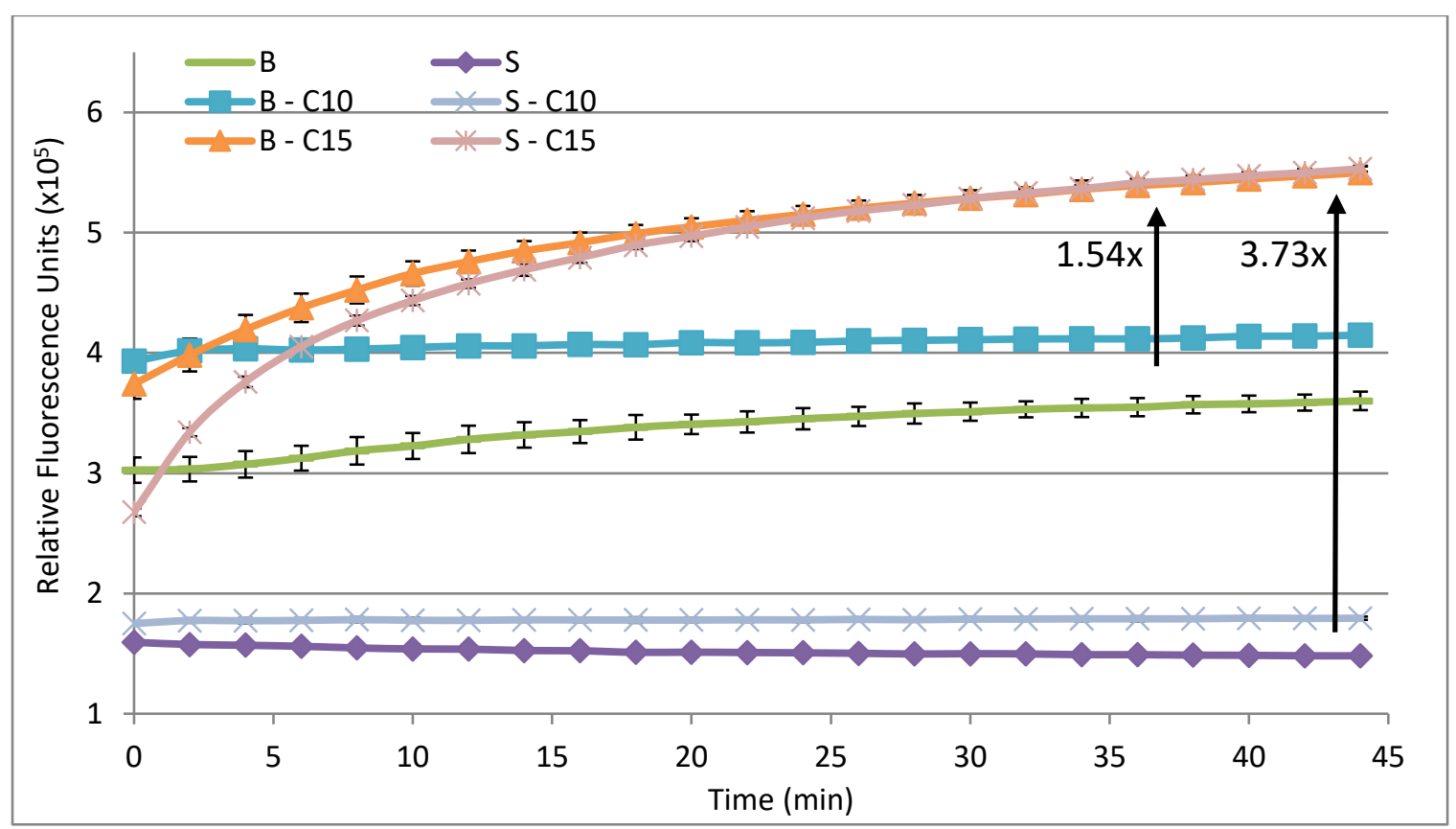

Figure 14 - Fluorescence intensity of fluorescein / dabcyl labeled aptamer beacon with 10 complementary bases (A10) $(1 \mu \mathrm{M})$ in the presence of C10 or 15 base complement (C15) (1 $\mu \mathrm{M})$ in TEB (B) and TES (S). Fluorescent control values were about $33 \times 10^{5}$ RFUs. Data points are the average of 4 wells (B and S), 5 wells (S-C10), and 6 wells (others). Error bars represent SD. Ex. 495 $\pm 10 \mathrm{~nm}, \mathrm{Em} .528 \pm 20 \mathrm{~nm}$.

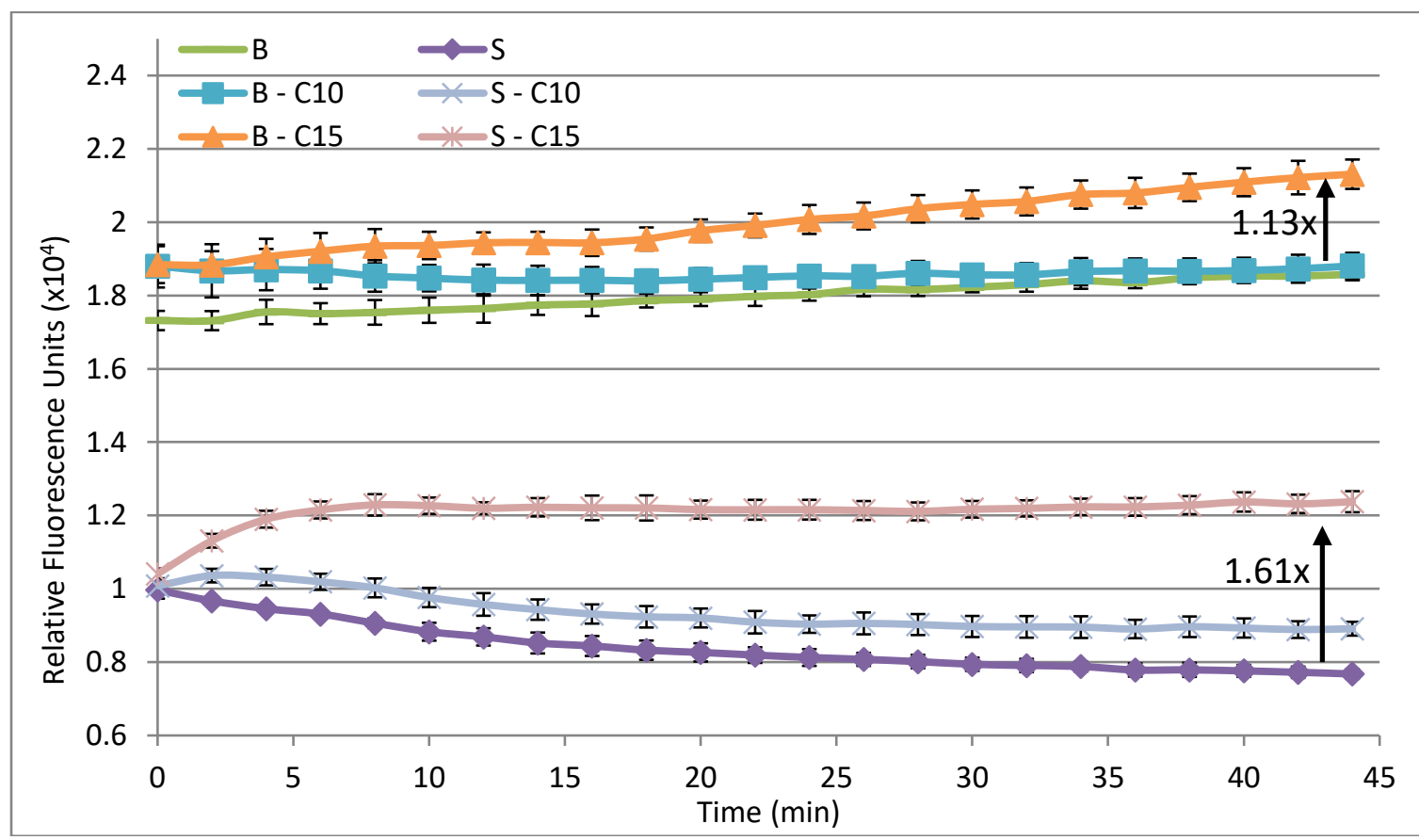

Figure 15 - Fluorescence intensity of fluorescein / dabcyl labeled aptamer beacon with 10 complementary bases (A10) (50 nM) in the presence of C10 or 15 base complement (C15) (50 $n M)$ in TEB (B) and TES (S). Fluorescent control values were about $12 \times 10^{4}$ RFUs. Data points are the average of 6 wells except $B$ and $S$ which had 4 wells. Error bars represent SD. Ex. $495 \pm 10$ $\mathrm{nm}, \mathrm{Em} .528 \pm 20 \mathrm{~nm}$. 
duplicate and replicated on a separate plate. Samples of beacon ( $50 \mathrm{nM}$ ) were prepared and mixed with VT1, VT2, or C15 at a 1:1 ratio in TEB. Readings were taken every two minutes and the experiment was repeated for both A10 (Figure 16) and A15 (Figure 17). C15 was used as a positive control showing a significant increase in fluorescence intensity in both cases $(p=0.01)$ although signal continued to increase for A10. There was a significant increase in fluorescence of A10 and A15 caused by VT1 as early as 46 and 26 minutes, respectively $(p=0.05)$. The increase was not as large as the increase caused by C15 for either beacon, but VT1 caused an increase almost as great as C15 in A15. VT2 did not show any increase in fluorescence, but caused a significant decrease compared to the background fluorescence $(p=0.05)$. VT1 curves appeared stable across the 60-minute period while background and VT2 curves show a steady decrease over the time period.

\subsubsection{Visibility of Fluorescein-Tagged Sequences on TSA}

To determine what concentration level fluorescein would be visible on agar plates, solutions of FC were prepared at $50,5,0.5$, and $0.05 \mu \mathrm{M}$. In solution, fluorescence was clearly visible at $50 \mu \mathrm{M}$, fairly visible at $5 \mu \mathrm{M}$, difficult to see at $0.5 \mu \mathrm{M}$, and not visible at $0.05 \mu \mathrm{M}$. On TSA plates, only $50 \mu \mathrm{M}$ spots were visible (not shown). Solutions of FC were prepared at 50, 40, 30, 20, and $10 \mu \mathrm{M}$ (Figure 18). Each of these solutions had visible fluorescence, although $10 \mu \mathrm{M}$ was difficult to see. $10 \mu \mathrm{L}$ spots of the FC solutions were placed on a TSA plate (Figure 19). 50, 40, and $30 \mu \mathrm{M}$ spots were clearly visible, $20 \mu \mathrm{M}$ spots were very difficult to see. The TSA plates produced a slight, 


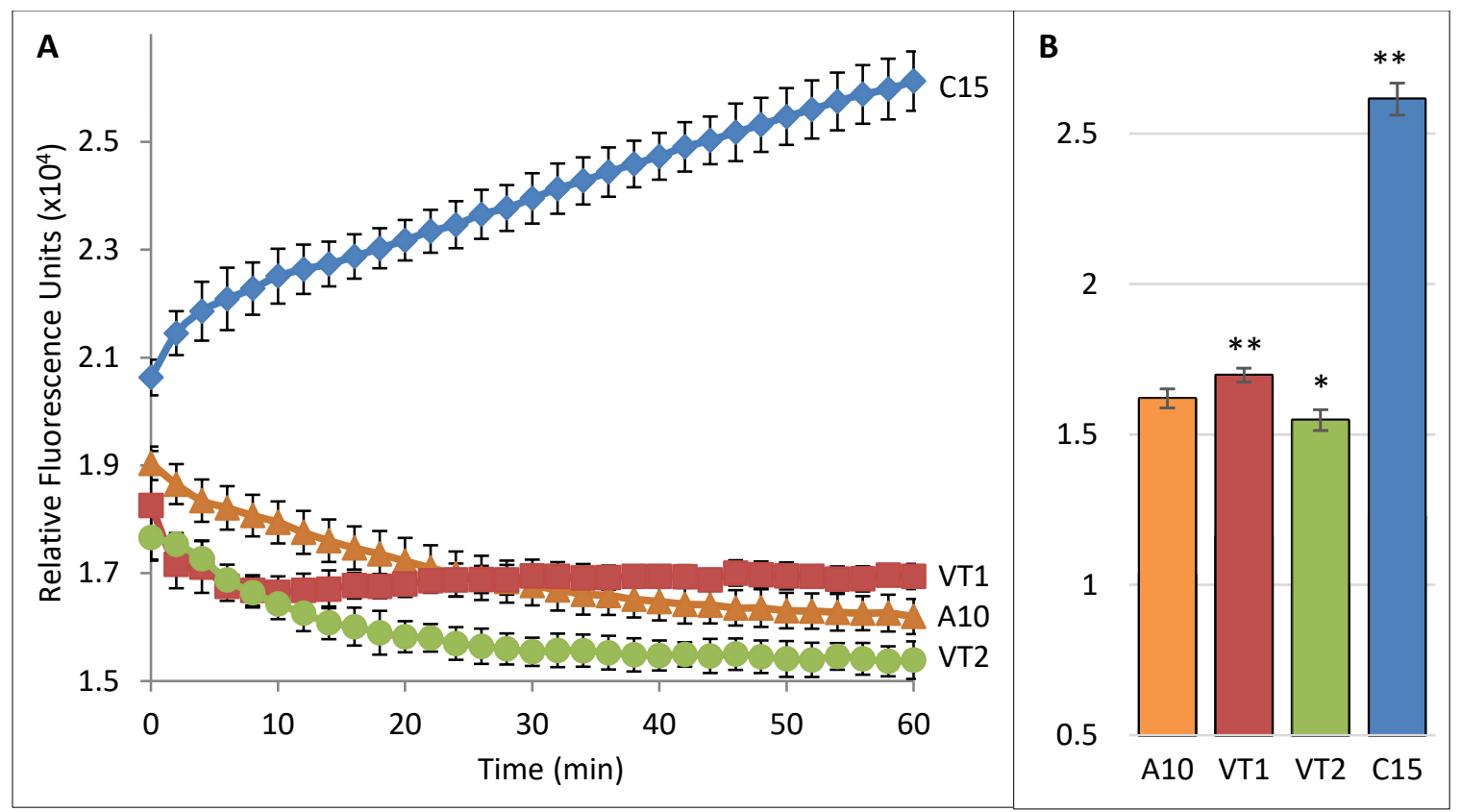

Figure 16 - Fluorescence intensity results for fluorescein / dabcyl labeled aptamer beacon with 10 complementary bases (A10) (50 nM) testing at a 1:1 ratio with C15, VT1a, and VT2a in TES buffer. A) results over 60 minutes; $B)$ Results at 60 minutes. * and ** values significantly different from background $p=0.05$ and $p=0.01$, respectively. Fluorescent control DNA (FC) values were about $13 \times 10^{4}$ RFUs. Data points are the average of 16 wells (A10 and C15) and 4 wells (VT1 and VT2) over two replicates. Error bars represent SD. Ex. $495 \pm 10 \mathrm{~nm}$, Em. $528 \pm 20 \mathrm{~nm}$.

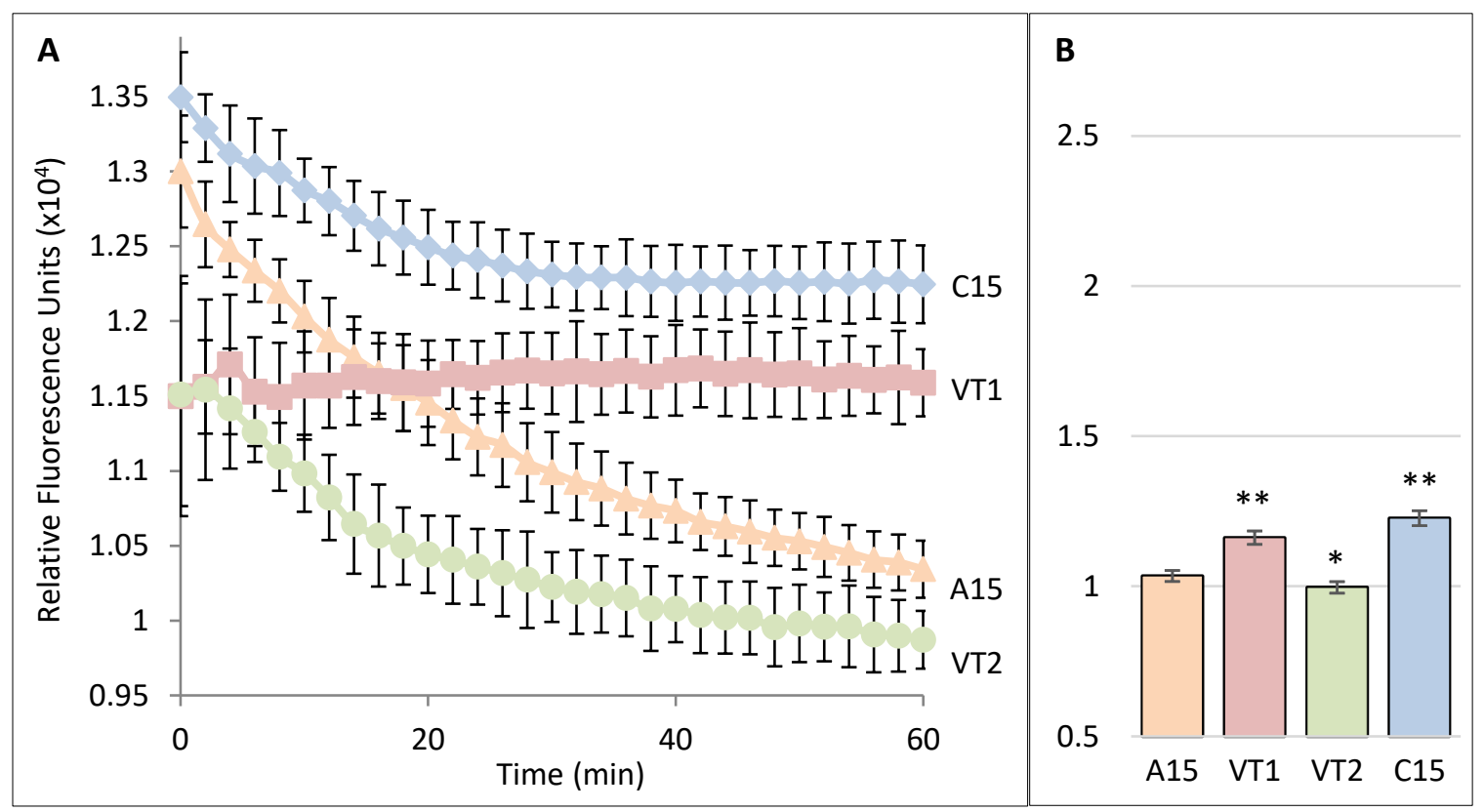

Figure 17 - Fluorescence intensity results for fluorescein / dabcyl labeled aptamer beacon with 10 complementary bases (A15) (50 nM) testing at a 1:1 ratio with C15, VT1a, and VT2a in TES buffer. A) results over 60 minutes; $B)$ Results at 60 minutes. * and ** values significantly different from background $p=0.05$ and $p=0.01$, respectively. Fluorescent control DNA (FC) values were about $13 \times 10^{4}$ RFUs. Data points are the average of 16 wells (A10), 14 wells (C15), and 4 wells (VT1 and VT2) over two replicates. Error bars represent SD. Ex. $495 \pm 10 \mathrm{~nm}, \mathrm{Em} .528 \pm 20 \mathrm{~nm}$. 
green background fluorescence, and in pictures reflected large amounts of UV light. Two plates with $\mathrm{A} 10(50 \mu \mathrm{M})$ and $\mathrm{C} 10(100 \mu \mathrm{M})$ were prepared with $30 \mu \mathrm{M}$ FC control (Figure 20). Fluorescence was seen for the FC control spots, but no fluorescence was observed from the test spots. Spots with A10 were slightly orange, the colour dabcyl produces normally.

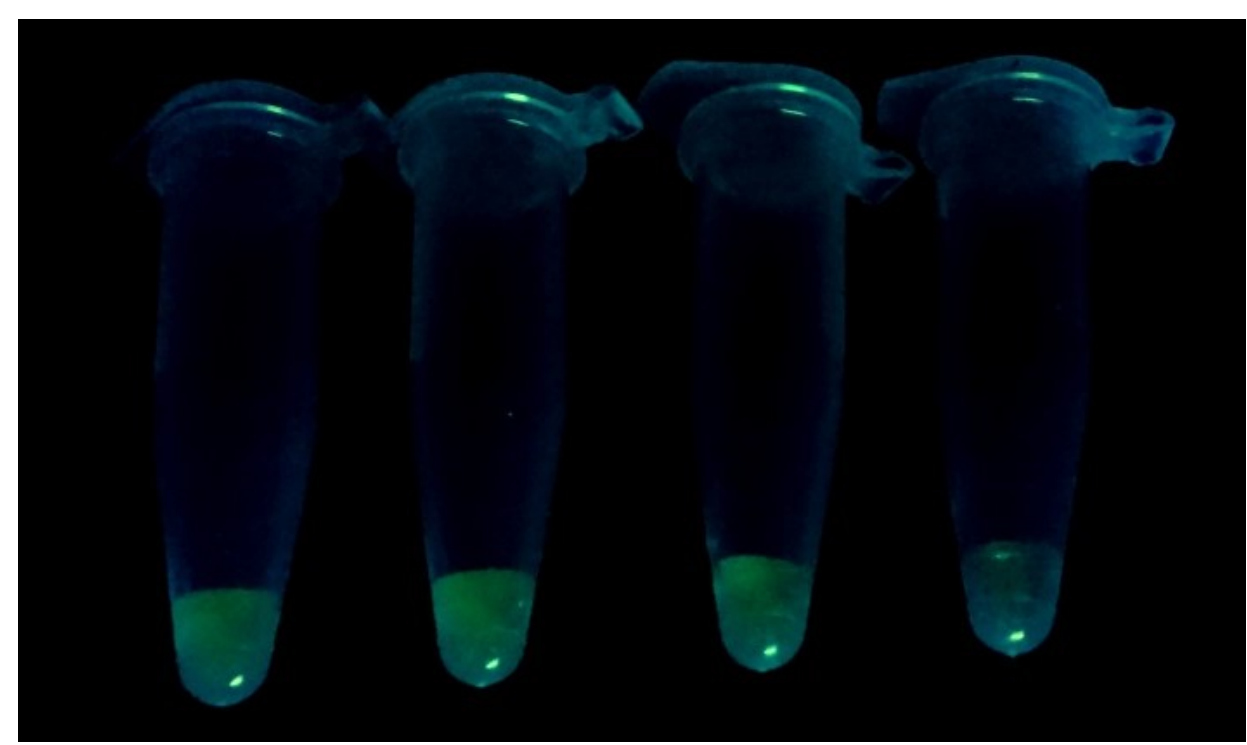

Figure 18 - Solutions of fluorescent control DNA (FC) at concentrations of 40, 30, 20, $10 \mu \mathrm{M}$ under UV lamp. Image enhanced.

\subsection{Discussion}

\subsubsection{Aptamer Beacon Design}

Little secondary structure was predicted for the unmodified 40-base randomized region of Fan et al.'s forward (+) aptamer (Figure 7A). The presence of additional structure could either make binding to a target molecule more difficult if the structure was not used in binding, or potentially beneficial to form a stable binding pocket without much reorganization. For aptamer beacons a hybridized region is added and selective signal is produced by a higher affinity for target molecule than for the hybrid sequence 


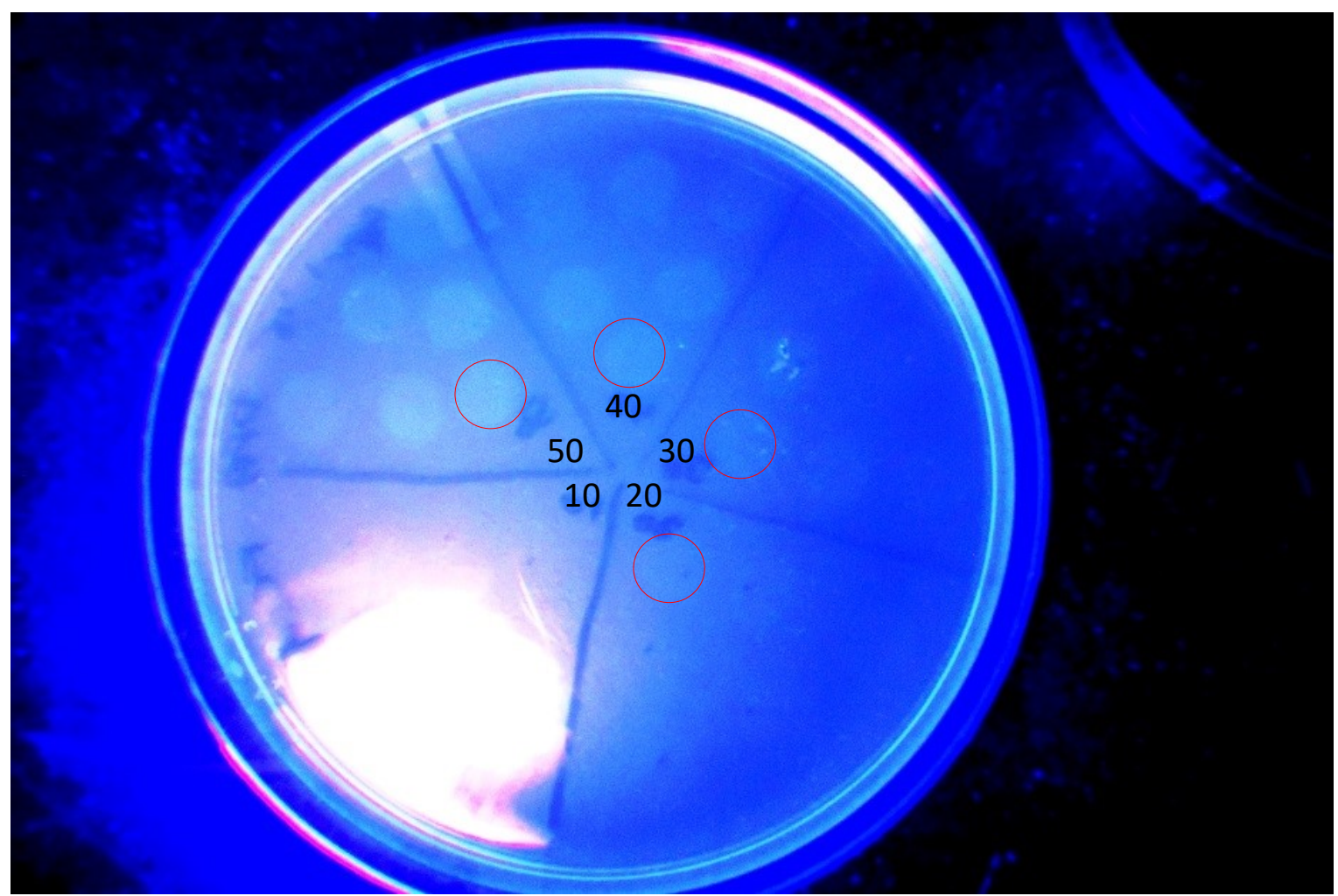

Figure 19 - Handheld UV lamp-illuminated TSA plate spotted with six spots of $10 \mu \mathrm{L}$ fluorescein labeled DNA at each of 50,40,30,20, and $10 \mu \mathrm{M}$. Image enhanced to reflect what was seen by eye. Red circles highlight examples of visibly fluorescent fluorescent control DNA (FC) spots.

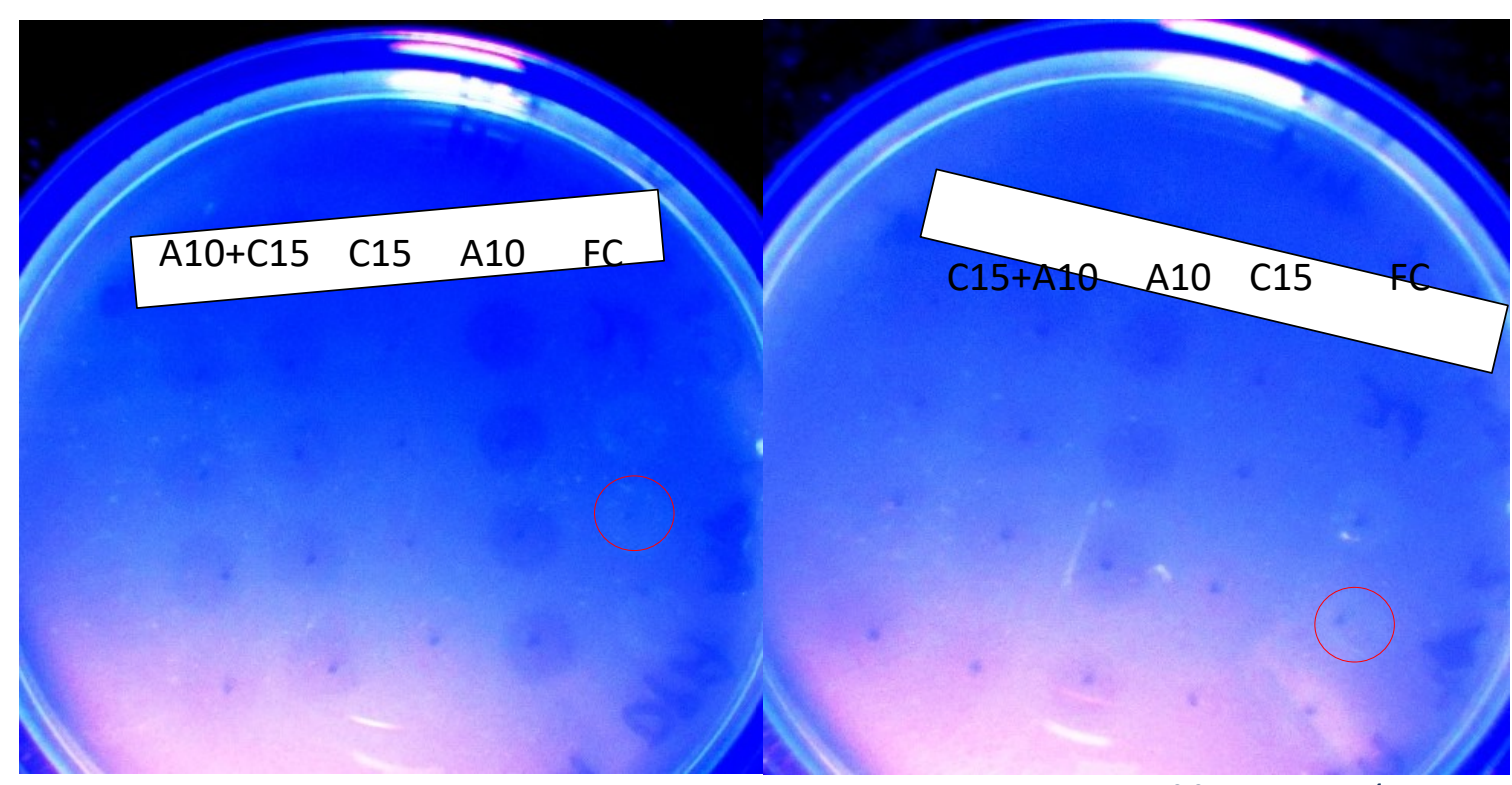

Figure 20 - Handheld UV lamp-illuminated TSA plates spotted with $10 \mu \mathrm{L}$ of fluorescein / dabcyl

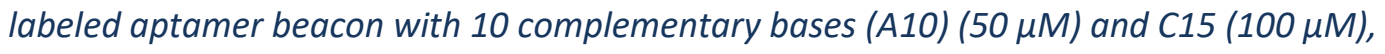
together, separately, and fluorescent control DNA (FC) (30 $\mu \mathrm{M})$. Left: A10 spotted first; right: $C 15$ spotted first. Images enhanced to reflect what was seen by eye. Red circles highlight a visibly fluorescent control spot. 
(Hamaguchi et al., 2001). The hybridization allows the fluorophore / quencher pair to be proximal so that under normal conditions fluorescence is not observed until a stronger binding interaction causes a change to the conformation (Nutiu and Li, 2004).

The modified aptamer beacon sequence with 10 or 15 additional complementary bases to the 5' end caused formation of a hybridized hairpin structure of 12 and 15 bases, respectively (Figure $7 \mathrm{~B}$ and $\mathrm{C}$ ). Since the exact number of complementary bases that would be required to yield a functional aptamer beacon was unknown, 10 and 15 bases were chosen to ensure a stable hairpin formation. By having a longer hybridized region, A15 would require a stronger interaction with the target to change conformation. Hamaguchi et al. proposed that essentially any aptamer could become an aptamer beacon by the addition of bases complementary to those important in target binding (2001). Unfortunately, there is no knowledge of how the Fan et al. (2008) aptamer binds, so potentially bases not involved in target binding are bound in the hybridizing region, which would lead to a poorly functioning probe.

For the fluorophore / quencher pair, fluorescein (Figure 6) was chosen as the fluorophore and dabcyl (Figure 5) was chosen as the quencher. This pair of molecules is used commonly in beacons and was readily available (Shi et al., 2011; Hamaguchi et al., 2001; Yamamoto and Kumar, 2000). Fluorescein has been well studied with papers published on various aspects of the molecule's chemical behaviour. Dabcyl has been viewed as a universal quenching molecule capable of quenching a wide range of fluorophores in addition to fluorescein (Tyagi et al., 1998). The pair has good spectral overlap and dabcyl has a 96-99.9\% quenching efficiency with fluorescein (Tyagi et al., 
1998). Many other options for fluorophore and quencher exist such as quantum dots, Texas Red, and Cy5 for fluorophores, and gold or silver nanoparticles, and black hole quenchers for quencher molecules (Resch-Genger et al., 2008; Schultz et al., 2000; Tyagi et al., 1998). Each of these could be used in various combinations provided the requirement for spectral overlap is met.

A complementary DNA sequence was also designed to test the aptamer beacons. By competing with the hybridizing region, it is expected that the beacon should change conformation, the stem loop should open. If the system is working as expected an increase in fluorescence intensity should be observed. This is the basic concept of molecular beacons (Nutiu and Li, 2004). The C10 complement was chosen since there were no predicted secondary structures which would inhibit binding to the aptamer beacons, while complements of 15 bases (Figure 8) or longer would have some minor predicted structures that could inhibit hybridization.

\subsubsection{Initial Fluorescence Testing with C10}

In the initial fluorescence testing with beacons $\mathrm{A} 10$ and $\mathrm{A} 15$, after 60 minutes an increase in fluorescence intensity was observed in all samples when C10 was added (Figure 9). A10 had higher fluorescence values than A15. This was likely due to the stronger binding of the hybridized region of A15. Since it forms a stronger bond it requires a stronger interaction to cause the conformation shift, or takes longer to affect the change. This was why $\mathrm{A} 10$ with $\mathrm{C} 10$ at time zero has a greater difference compared to background fluorescence, while the difference for A15 takes time to increase. 
Although the initial results indicated a measurable change of fluorescence in the presence of $\mathrm{C} 10$ a couple issues were present. The most significant issue was the high background fluorescence of the beacons alone in buffer $(80-90 \%$ of the resulting emission), as well as the increase in background signal over time. Some causes of this could be a loss of dabcyl molecules from the beacon, which was partially seen in the mass spectrometric results (4B and 6B in Appendix B). Additionally, the hairpin could be opening on its own. The second issue was that the resulting emission of the beacon was only about one ninth of the fluorescent control (FC) emission, indicating that very little signal was being induced.

Both present a problem due to the limited amount of toxin available for initial testing (10 $\mu \mathrm{g}$ of each). To conserve the toxin the conditions needed to be optimized in order to decrease background fluorescence and increase the resulting change in emission. Additionally, confirmation that the tests could be conducted at lower concentrations of beacon and toxin were required. To optimize the system, A10 which was observed to produce higher fluorescence intensities, was used to check the effects of temperature, beacon refolding conditions, buffer salinity, complement length, and concentration upon the background fluorescence and resulting emission.

The effect of $\mathrm{pH}$ in solution was not addressed in this study. Studies typically mention that fluorescence of fluorescein is strongly $\mathrm{pH}$ dependent, but that $\mathrm{pHs}$ above 7 are optimal (You et al., 2011). With the buffer balanced to 7.4 and toxin requiring physiological pH for proper protein folding, this factor was not investigated. Another factor that could be investigated is the presence of metal ions such as $\mathrm{Mg}^{2+}$, which were 
not present in the buffer but can influence aptamer folding (Hamaguchi et al., 2001).

The linear range of fluorescence intensity was found to be $2.5 \mathrm{nM}$ to $5 \mu \mathrm{M}$ (Figure A-3). Concentrations of beacon of $10 \mathrm{nM}, 100 \mathrm{nM}$, and $1 \mu \mathrm{M}$ would be within the linear detection range of fluorescein.

\subsubsection{Melting Temperature Determination and Effect of Temperature}

The melting temperatures of the beacons were assessed because there was a possibility that the beacons were able to denature naturally over time at room temperature, resulting in increased background fluorescence. Based on the melting temperatures of $\mathrm{A} 10$ and $\mathrm{A} 15$ of $42.2 . \pm 0.6^{\circ} \mathrm{C}$ and $44.8 \pm 0.5^{\circ} \mathrm{C}$, respectively (Figure 10 ), it is unlikely that temperature contributed to this problem. At room temperature, the beacons should be at an equilibrium, which should have been reached during the 30 minutes of cooling. It can be predicted that A15 would have a slightly higher melting temperature since it has a longer hybridized DNA region. Increasing the temperature of the system above $40{ }^{\circ} \mathrm{C}$ would not be expected to improve emission intensity of the beacons. This could result in higher background fluorescence by minimizing difference to the melting temperatures.

A decrease in fluorescence was observed at higher temperatures (Figure 11). This was due to a higher instability of fluorescein at higher temperatures with higher temperatures enhancing decay rates. A decrease of $30 \%$ in a $20 \mathrm{mM}$ solution (TEB) was observed (Figure 11). In a $10 \mathrm{mM}$ salt buffer at $60^{\circ} \mathrm{C}$ about a $20-30 \%$ decrease in fluorescence expected (Liu et al., 2005). Although an increase of $90 \%$ fluorescence for 
the beacon was observed, this was due to the hairpin melting, and the value was actually lowered by the decreased emission of fluorescein at higher temperatures. Together this indicated that working at lower temperatures would be ideal for the system to read fluorescence emission values more accurately.

\subsubsection{Effect of Reannealing Media}

Refolding conditions were investigated since improper folding would result in a higher background. The reason refolding is done in aptamer experiments is to dissociate any potential intermolecular structures that may have formed between the various strands of aptamer in solution; allowing the structure with thermodynamic minimum to be present during experimentation. Salt ions often play a role in the stability of DNA conformations, prompting the investigation of the difference between refolding in water or in buffer (Nakano et al., 1999).

A decrease of about $10 \%$ background fluorescence following heating in buffer was observed (Figure 12). This likely resulted from A10 being able to fold into a slightly more stable conformation. However, the results in buffer varied from about $10 \%$ decrease to a slight increase observed at lower concentrations (Figure A-9 and Figure A-10). A similar decrease was observed in the resulting emissions (Figure 12). Although minimal, heating in buffer was optimal for the beacon function during experimentation. Since the effect was variable further testing was desired. 


\subsubsection{Effect of Buffer Salinity}

The effect of buffer salinity on background and emission fluorescence was investigated by experiments in a low salt buffer (TEB) and a high salt buffer (TES). Dabcyl has been shown to quench fluorescein more effectively in high salt buffers (Dubertret et al., 2001) and so this was a way to potentially decrease the background fluorescence. Little difference in background between refolding in TES or water was observed (Figure A-8, Figure A-9, and Figure A-10).

TES was a more suitable medium for experiments since over the 60-minute incubation the background fluorescence did not increase in TES (Figure 13). This was likely due to the increased stability of the beacon system in higher salt (Nakano et al., 1999). It is known that increased $\mathrm{K}^{+}$in buffer promotes duplex formation (Hamaguchi et al., 2001) which would enhance the stability of the complement region. This stability could explain the longer reaction time with $\mathrm{C} 10$. Whereas the beacon quickly increased in fluorescence in TEB, in TES the fluorescence intensity continued to increase slowly over a 60-minute incubation.

However, a consistent decrease in fluorescence of about $40-50 \%$ in both background and resulting emission was observed. This was due to dabcyl more effectively quenching in the higher salt TES buffer (Dubertret et al., 2001). However, if the resulting emission intensity was decreased either dabcyl was not being displaced very far from fluorescein, or the concentration of dabcyl molecules in the solution was causing quenching regardless of conformation changes. Similar effects were seen at lower concentrations, so the concentration of dabcyl in the solution was unlikely as a 
cause of this effect. The effect of not changing conformation could be investigated by using a complete complement to ensure beacon conformation change.

Although both the background and resulting emission were decreased by about $45 \%$ by higher salt content in the buffer, over the 60 -minute period the background was more stable in TES and the resulting emission continued to increase (Figure 13). Combined, TES seemed to be a better choice of buffer to assist in differentiating the background from the resulting emission with difference being best observed over an incubation period.

\subsubsection{Effect of Complement Length}

In each of the optimization trials A10 was used, however, the hairpin of A10 was actually 12 base pairs long (Figure 7B). C10, which is 10 bases long, appeared to be inefficient at opening the beacon since the higher salinity decreased the resulting emission as much as the background fluorescence. Although a complement with more bases would have some secondary structure (Figure 8) it would have a higher likelihood of opening the A10 hairpin.

In TES a complement with 15 bases (C15) affected a much larger increase in fluorescence intensity, matching the TEB C15 values (Figure 14). This showed that A10 was undergoing a major conformation change, while C10 may have only affected a minor change. The fact that the values were equal was not surprising, since the same amount of beacon was present in each scenario. Under a major conformation change both TEB and TES should have the same resulting emission. These results support the 
idea that $\mathrm{C} 15$ is much more effective at competing with the hybridized region and that TES is a more optimal buffer for increasing the difference between the background and resulting emission. Additionally, the increased stability under high salt conditions was still seen with it taking more time for the fluorescence emission to reach the same levels for the TES sample. This showed that the added salt was not actually causing quenching.

Due to limited toxin available, the toxin would have to be tested at 1:1 ratio at 50 nM. Previously $1: 1,1: 2$, and 1:4 ratios of beacon to complement resulted in similar emission intensity, so it was expected that using a 1:1 A10: toxin ratio would not be an issue. However, if more toxin was available, investigating the effect of the beacon: target ratio would be possible. Under these conditions $\mathrm{C} 15$ was shown to be a much better positive control for the opening of the A10 beacon (Figure 15).

The increase in fluorescence caused by $\mathrm{C} 15$ at $50 \mathrm{nM}$ in TES did not match that in TEB. This was likely due to the lower concentration of both beacon and complement in the samples. At lower concentrations it would be less likely for the individual molecules to interact. However, the increased fluorescence signal was still larger than for TEB at 60 minutes (1.13x vs 1.61x for TEB and TES, respectively).

\subsubsection{Effect of Concentration}

All previous experiments were conducted at multiple concentrations resulting in similar results at each. Thus, the optimized conditions could be applied for testing the effect of toxin on induced fluorescence at concentrations of $50 \mathrm{nM}$. 


\subsubsection{Effect of Toxin on Probe Fluorescence}

Interaction between VT and beacons was performed at $50 \mathrm{nM}$ and 1:1 ratio due to limited toxin. Both A10 and A15 were used to see if hairpin length had an effect on the interaction. For A10 the VT1a toxin elucidated only a small response, but indicated a significant affinity $(p=0.05)$ (Figure 16). However, for A15, a greater difference was observed after 60 minutes ( $p=0.01$ ) (Figure 17). For both beacons, a stable interaction was formed, as evidenced by the stable fluorescence intensity across the incubation period. The relative difference between background and VT1 signal showed that the complementary base number influences the beacons' ability to interact with the target, and as such is a parameter which could be optimized in the future. Other published aptamer beacons have complement lengths ranging from 4 to 9 bases (Shi et al., 2011; Hamaguchi et al., 2001; Tyagi et al., 1998). Tyagi and Kramer (1996) have suggested that the target-binding sequence in the aptamer should be at least twice the length of the hairpin sequence and that the fluorophore and quencher molecules should be attached to bases in the stem, not at the sequence termini. The exact location of interaction between the aptamer and VT is unknown, however, but could be determined using mutation or partial sequence analysis.

For VT2a both beacons showed a significantly decreased fluorescence $(p=0.05)$ that followed a similar fluorescence decay curve as the background. This was likely caused by general quenching by amino acid residues in the protein such as histidine, methionine, tryptophan, and tyrosine (Chen et al., 2010). Likely, a similar degree of protein-based quenching was caused by interaction with VT1 since they share a high 
proportion of peptide sequence. This may be facilitated by the design of the aptamer beacon. Assuming the interaction of the beacon and protein does occur, fluorescein is held close to the toxin while dabcyl is further removed by the complement region. Reversing the position of fluorescein and dabcyl (or placement of the complement) could potentially increase signal. Additionally, the use of different fluorophores could also potentially generate a larger relative signal increase for better detection potential (Chen et al., 2010). General non-specific protein quenching should be further investigated. If it is found to be a problem, use in a complex solution may be problematic. Additionally, the beacon was not seen to interact with VT2. With VT2 being the toxin type more strongly associated with severe disease modification of an aptamer sequence targeting VT2 would be highly desired for detection of high risk VTEC.

C15 was used as a positive control since it had been demonstrated to cause strong increase fluorescence intensity of A10. The resulting fluorescence of A15 with C15 was lower than A10 due to the longer hairpin. Additionally, C15 only stabilized for A15, compared to the continually increased signal with $A 10$. As $\mathrm{C} 10$ had difficulty causing A10 to open previously, so A15's hairpin was more difficult to open and even a complement of 15 bases could only compete with the hybridized region.

Assuming that interaction is occurring in the hairpin, comparing the affinity of the beacon to C15 or VT1 offers a means of comparing the aptamers' affinity for the verotoxin. For A10, which has a 12-base hairpin, C15 easily caused a strong increase in signal, while VT1 only caused stabilization of background signal. As such there must be a stronger affinity for $\mathrm{C} 15$. For A15, which has a 15-base hairpin, $\mathrm{C} 15$ caused a slight 
increase in signal that then followed a similar decay curve which stabilized more than the background, while VT1 causes an immediate drop in signal that then remained fairly stable increasing slightly before decaying slightly. While accounting for the initial protein-based quenching, there would still be a stronger increase in signal from C15, but less decay with VT1. This further supports the hypothesis that the beacon sequence has a higher affinity for C15. However, the increased stability and immediacy of the interaction suggests that the interaction with the protein was more stabilizing to the fluorescein. Very likely, however, this low level of fluorescence could be due to the hairpin not fully opening, meaning the fluorophore and quencher were not fully separated (Rajendran and Ellington, 2003). Potentially only bases in the loop region were interacting, which would not cause the hairpin to open. This could be investigated using analysis of aptamer beacons prepared with complements to the different regions of the sequence. Alternatively, there is a possibility that some of the bases which were in the primer-binding regions of the aptamer sequence play a role in binding to the toxin. As such, removing them may have caused the reduced affinity observed here. This could be investigated with aptamer beacon preparations with varying portions of the full aptamer sequence. Finally, there is a chance that binding does not cause a change in structure. In that case a new sequence would be required. A selection incorporating a structure switching selective pressure could be used to ensure sequences sufficiently change conformation in the presence of toxin (Rajendran and Ellington, 2003).

Other fluorescein / dabcyl aptamer beacons have been published. A beacon prepared for a thrombin aptamer $\left(K_{d}=10 \mathrm{nM}\right)$ showed an increase in signal of $2.5 \mathrm{x}$ with 
saturating target levels and $8 x$ increase in the presence of a complete complement sequence (18 bases) (Hamaguchi et al., 2001). Non-specific binding with single-stranded binding proteins or lactate dehydrogenase led to increase in signal of 15x (Hamaguchi et al., 2001). Another aptamer beacon for HIV Tat protein was designed to be in two parts. It had a signal increase of about $9 x$ at equal concentrations and $14 x$ with double the target, while a full complement resulting in about 23x increase in signal (Yamamoto and Kumar, 2000). Optimally designed beacons can produce a 200X increase in fluorescence while more common increases are in the range of 10-60x (Rajendran and Ellington, 2003). These values support very strongly that the current beacon was not opening as desired.

\subsubsection{Visibility of Fluorescein-Tagged Sequences on TSA}

The aptamer beacon was tested to determine if it could be used to detect VTEC growing on enrichment media. FC was used to determine what concentration level could be seen without imaging technology. Although levels of fluorescence could be seen to some degree at $0.5 \mu \mathrm{M}$ in solution, once plated, the spots of $5 \mu \mathrm{M}$ solution were not able to be distinguished as fluorescent (not shown). Part of that was due to the background fluorescence of the agar plate which was similar to the green colour of the fluorescence produced by fluorescein, making fluorescence difficult to discern.

Solutions down to $10 \mu \mathrm{M}$ were prepared (Figure 18) and fluorescence was visible at $30 \mu \mathrm{M}$ on TSA plates (Figure 19). $20 \mu \mathrm{M}$ was somewhat visible. As a quick test, the highest concentration of $\mathrm{A} 10$ and $\mathrm{C} 15$ solutions that were on hand were spotted, 50 and 
$100 \mu \mathrm{M}$ respectively (Figure 20). The FC control was visibly fluorescent, but the combination of A10 and C15 was not in either case. All testing so far previously had only ever produced a fluorescence intensity about one sixth of FC at the same concentration. It may be that the two were interacting and fluorescing, just not at a visibly discernable level on the TSA plate. Further optimization of the aptamer beacon system, and the fluorophore / quencher pair may lead to such agar tests in the future.

\subsection{Conclusions}

Following optimization of experimental conditions, the internal beacon system created using the randomized region of Fan et al.'s forward (+) aptamer sequence consistently showed measurable and significantly increased fluorescence in the presence of complementing DNA. The signal was optimal at room temperature after refolding in a high salt buffer. The signal increase was more significant with longer complements, however it did not approach the maximum fluorescence observed by a non-quenched fluorescein labeled DNA sequence. In the presence of VT1a, both A10 and A15 showed a significant interaction resulting in significantly higher fluorescence compared to background after 60 minutes $(p=0.05)$, while VT2a caused a significant decrease $(p=0.05)$. This showed that there was a significant interaction between the aptamer sequence and VT1, which had not previously been shown. The system showed promise for aptamer beacons for VT, although it was likely that the interaction did not result in a major conformational change in the beacon. Investigation to determine the bases interacting with toxin would allow for better aptamer beacon design with 
complement bases for the target binding bases. In that case a greater increase in signal would be expected. Finally, the fluorescence produced by fluorescein was difficult to visualize, and the aptamer beacon system as it is now could not be observed on standard TSA media plates. Future characterization of the aptamer sequence, optimization of the beacon system, and potential selection of a novel structure switching aptamer sequence would be of high value to VT detection by aptamer beacons. 


\subsection{Further Characterizing the Aptamer Beacon}

\subsection{Introduction}

\subsubsection{Characterization of Aptamer Beacons}

Aptamer beacons offer the potential for specific real-time detection of target molecules. These are frequently modified after aptamer selection by addition of fluorophores and small complementary regions. Aptamers chosen for modification display favourable characteristics, allowing for strong interaction with the target molecule. Following selection, aptamers are characterized to determine their affinity and specificity for the target molecule among other parameters. The verotoxin (VT) DNA aptamer selected by Fan et al. was published without characterization information (Fan et al., 2008). Aside from the nucleotide sequence no additional information characterising the aptamer was provided. Initial proof of concept experiments have shown that the aptamer interacts with VT1a and not VT2a. However, there is still no quantitative information on the strength of the interaction, or potential interaction with other proteins. Of additional interest is that the location of interaction on the toxin is not known and its $A B_{5}$ structure may allow for aptamer interaction at stoichiometries greater than 1:1.

The strength of interaction between aptamers and their targets is measured by their affinity. Generally, the dissociation constant, $K_{d}$, is the quantitative value used to measure affinity. The smaller the $K_{d}$ the stronger the binding interaction. There are a number of methods that can be used to measure aptamer affinity ranging from elutions, filtrations, probing, labeling, surface plasmon resonance, and isothermal titration 
calorimetry, among others (Chang et al., 2014). Each method has its strengths and weaknesses. For example, fluorescent labeling of the aptamer has potential to be simple and sensitive, but may affect the affinity of the interaction by way of the required modifications.

Practically, the signal contrast between background noise and induced signal, as well as the ability of the aptamer beacon to function in the desired testing medium are also important characteristics to be determined for the Fan et al. aptamer beacon.

\subsection{Chapter Objectives}

Following the conclusions found in the proof of concept experiments, further testing of the fluorescein and dabcyl labeled beacon was conducted to determine if the beacon could be used to detect VT1 in buffer or complex media. Additionally, further characterization of the aptamer sequence was performed to determine specificity of the interaction.

\subsection{Statement of Contributions}

Determination of linear fluorescent range, potential fluorescence, interaction with VT, limit of detection with FLQ, fluorescence in TSB, and fluorescence with crude toxin experiments were performed by Daniel Brumar. Mass spectrometric analysis performed by Alexander Wahba, McGill University, Mass Spectrometry Facility. Crude VT was prepared by Mary Rao. 


\subsection{Materials and Methods}

\subsubsection{Buffers and Broth}

Table 5-Buffers and broth used in Chapter 3.0 - Further Characterizing the Aptamer Beacon.

\begin{tabular}{|c|c|}
\hline Name & Components \\
\hline TES (tris-EDTA high salt) & $\begin{array}{c}10 \mathrm{mM} \text { tris, } 0.5 \mathrm{mM} \text { EDTA, } 0.5 \mathrm{M} \mathrm{NaCl}, 0.5 \mathrm{M} \mathrm{KCl} \text {, } \\
\mathrm{pH} 7.4 \text { (adjusted with } \mathrm{HCl} \text { and } \mathrm{NaOH} \text { ) }\end{array}$ \\
\hline $\begin{array}{l}\text { Phosphate buffered } \\
\text { saline (PBS) (0.2 M, 1L) }\end{array}$ & $\begin{array}{l}\mathrm{NaH}_{2} \mathrm{PO}_{4} \cdot \mathrm{H}_{2} \mathrm{O} 19 \mathrm{~mL}, \mathrm{Na}_{2} \mathrm{HPO}_{4} \cdot 12 \mathrm{H}_{2} \mathrm{O} 81 \mathrm{~mL}, \mathrm{NaCl} 8.5 \mathrm{~g} \text {, } \\
\quad \mathrm{pH} 7.4\end{array}$ \\
\hline Tryptic soy broth (TSB) & $\begin{array}{l}17.0 \mathrm{~g} / \mathrm{L} \text { pancreatic digest of casein, } 3.0 \mathrm{~g} / \mathrm{L} \text { papaic digest of } \\
\text { soy, } 14 \mathrm{mM} \text { dextrose, } 86 \mathrm{mM} \mathrm{NaCl}, 14 \mathrm{mM} \mathrm{K}_{2} \mathrm{HPO}_{4} \text {, } \\
\mathrm{pH} 7.4 \text { (adjusted with } \mathrm{HCl} \text { and } \mathrm{NaOH} \text { ) }\end{array}$ \\
\hline VT elution buffer & $50 \mathrm{mM}$ tris, $250 \mathrm{mM} \mathrm{NaCl}, \mathrm{pH} 10$ \\
\hline $\begin{array}{l}\text { 10x SDS-PAGE running } \\
\text { buffer (1L) }\end{array}$ & 30.3 g Tris-base, 144.0 g glycine, 10.0 g SDS \\
\hline $\begin{array}{l}2 \times \text { SDS protein sample } \\
\text { buffer }(10 \mathrm{~mL})\end{array}$ & $\begin{array}{l}1.25 \mathrm{ml} 1 \mathrm{M} \text { Tris-HCl, } 4.0 \mathrm{ml} 10 \% \text { (w/v) SDS, } 2.0 \mathrm{ml} \text { glycerol, } \\
0.5 \mathrm{ml} 0.5 \mathrm{M} \text { EDTA, } 4 \mathrm{mg} \text { bromophenol blue, } 0.2 \mathrm{ml} \mathrm{b} \text { - } \\
\text { mercaptoethanol (14.3 M), pH } 6.8\end{array}$ \\
\hline
\end{tabular}

\subsubsection{DNA Synthesis, purification, and quantification}

\subsubsection{Prepared DNA}

DNA was synthesized and purified as described previously in section 2.4.3.

Sequences synthesized were as follows where the underlined sections are

complementary to form a hairpin and quenched sequences were modified with

quencher (in brackets):

CC: 5'-GGT AAC TAG CAT T+AT AT GGA CGG GAC GGG TGT GGG AAA TGA ATG CTA GTT ACC-3'

FLX (FLQ): 5'-Fluorescein-GGT AAC TAG CAT TCA TTT CCC ACA CCC GTC CCG TCC AT AT+A ATG CTA GTT ACC-(Dabcyl-)3'

\subsubsection{Fluorescent Measurements}

Fluorescence measurements of samples were performed using a Synergy 2

microplate reader (Biotek) with Gen5 software. Readings were taken with optical filters: 
$495 \pm 10 \mathrm{~nm}$ for excitation, and $528 \pm 20 \mathrm{~nm}$ for emission. A dichroic "top $510 \mathrm{~nm}$ " mirror and a sensitivity setting of 90 was used throughout.

DNA samples were prepared by dilution to the desired concentration. Samples were heated at $90{ }^{\circ} \mathrm{C}$ for 10 minutes and allowed to cool at room temperature for 30 minutes before mixing with toxin. Samples were plated in $200 \mu \mathrm{L}$ aliquots on black 96well black microtiter plates (Nunc) for testing.

\subsubsection{Linear Range of Fluorescence}

Samples of FLX were prepared at concentrations of 0.0025 to $5000 \mathrm{nM}$ in TES buffer and plated in triplicate. Fluorescent readings were taken. Three replicates of the experiment were performed on separate days. After blank subtraction, the data were plotted on a log-log graph, fitted by least-squares linear regression using Microsoft Excel 2007 software. The LODs and LOQs from all three replicates were calculated and averaged to obtain final values.

\subsubsection{Determination of Potential Fluorescence}

Samples of FLX, FLQ, and FLQ+CC were prepared at concentrations of 0.025 to $5000 \mathrm{nM}$ in TES buffer. Solutions were then heated for 10 minutes at $90^{\circ} \mathrm{C}$, cooled for 30 minutes at room temperature, and plated in triplicate. Fluorescent readings were taken. Three replicates of the experiment were performed on separate days.

\subsubsection{Molecular Beacon Potential in TSB}

Samples of FLX were prepared to $10 \mathrm{nM}$, and samples of FLQ or CC were prepared at concentrations of $20 \mathrm{nM}$ in TES buffer or TSB media. FLQ and CC samples 
were diluted to $10 \mathrm{nM}$ or mixed to make a FLQ/CC solution at $10 \mathrm{nM}$. These samples were plated in triplicate. Fluorescent readings were taken.

\subsubsection{Interaction with VT}

Samples of FLX and FLQ were prepared in TES. FLQ samples were mixed at a 1:1 ratio with final concentrations of $10 \mathrm{nM}$ with VT1a, with VT2a, or without VT (List Biological Labs, Inc.; CA, USA). Samples were plated in triplicate. VT in buffer samples were plated only once per plate. Fluorescent readings were taken at $0,5,15,30$, and 60 minutes. Four replicates were performed on separate days.

\subsubsection{Limit of Detection with FLQ}

Solutions of FLX, FLQ, and VT1 were prepared. FLQ samples were prepared by mixing with VT1 at concentrations from 0.078 to $10 \mathrm{nM}$ or without VT with final FLQ concentrations of $10 \mathrm{nM}$ in TES buffer. Samples were plated in triplicate except for VT in buffer samples which were plated only once per plate. Fluorescent readings were taken at $0,5,15,30$, and 60 minutes. Four replicates were performed on separate days.

\subsubsection{Fluorescence in TSB}

Samples of FLX and FLQ were prepared in TSB. FLQ samples were mixed with VT1 or without VT at a 1:1 ratio with final concentrations of $10 \mathrm{nM}$. Samples were plated in triplicate. Fluorescent readings were taken at $0,5,15,30$, and 60 minutes. Two replicates were performed on separate days with the first excluding VT2 samples.

Serial dilutions of TSB from $10^{0}$ to $10^{-8}$ were prepared on a 96-well plate using TES. Each dilution had 9 samples. Free fluorescein (FI), FLX, and FLQ were prepared and 
added to each sample for a final concentration of $10 \mathrm{nM}$. The fluorescence was measured at $0,5,15,30$, and 60 -minute time points and the rate of fluorescence decay was determined for each. Three replicates were performed on separate days.

\subsubsection{Fluorescence with Crude Toxin}

A sample of crude VT1a toxin was previously prepared. The method employed was that of Mulvey et al., 1998. Briefly, an 0103:H2 VTEC strain was incubated and expression enhanced with Mitomycin C. Polymyxin B sulfate was used to lyse the cells, and cell debris was removed by centrifugation. Synsorb-P1 (disaccharide) was used to purify the toxin. Toxin was eluted using VT elution buffer. The toxin was concentrated and stored at $4{ }^{\circ} \mathrm{C}$. Protein concentration was determined using Pierce ${ }^{\mathrm{TM}}$ BCA Protein Assay Kit (Thermo Scientific; IL, USA) and Synergy 2 spectrophotometer (BioTek).

VT samples were prepared for sodium dodecyl sulfate-polyacrylamide gel electrophoresis (SDS-PAGE) by mixing $10 \mu \mathrm{L}$ of sample with $10 \mu \mathrm{L}$ Laemmli Sample Buffer (Bio-Rad) prepared with $\beta$ mercaptoethanol. The samples were heated for 10 minutes at $95{ }^{\circ} \mathrm{C}$. Samples were loaded into precast gel and run for about 60 minutes at a constant amperage ( $\sim 25 \mathrm{~mA})$. With gentle agitation, the gel was fixed for 15 minutes in fixing solution ( $50 \%$ methanol, $10 \%$ acetic acid), stained for 30 minutes in staining solution (0.1\% Coomassie Brilliant Blue R-250, 50\% methanol and 10\% acetic acid), destained for 15 minutes in destaining solution (40\% methanol, $10 \%$ acetic acid), washed for 20 minutes in water, destained for an additional 85 minutes.

Crude VT1a toxin samples (cVT) were diluted and mixed with FLQ. Samples of FLQ and FLQ+cVT were plated in triplicate. The fluorescence was measured every 
minute for 60 minutes. Three replicates were performed on separate days.

\subsubsection{Effect of non-specific protein quenching}

Samples of FLQ and FLX were prepared. VT, bovine serum albumin (BSA), and TSB solutions were prepared to a protein concentration of $20 \mathrm{nM}$. FLQ and FLX solutions were mixed with equal amounts of VT, BSA, TSB, or buffer for final concentrations of 10 nM. Fluorescence was measured every minute for 60 minutes. Three replicates were performed on separate days.

\subsubsection{Data Analysis}

Basic analysis of averages, confidence intervals, and standard deviations was done with Microsoft Excel software. Statistical analysis was also conducted using Microsoft Excel software at a significance level of $p=0.05$.

\section{$3.5 \quad$ Results}

\subsubsection{Aptamer Beacon Design and Synthesis}

The FLQ aptamer beacon was designed as previously, but with 13 complementary bases at the $3^{\prime}$ end of the sequence. FLX was the same as FLQ, but without dabcyl. CC was a complete complement sequence to FLQ without any modifications.

The inclusion of 13 complementary bases was predicted to result in folding of both FLQ (Figure 21) and CC (Figure 22) to form a hairpin. CC had the addition of small potential loops starting at bases 21 and 31 . 


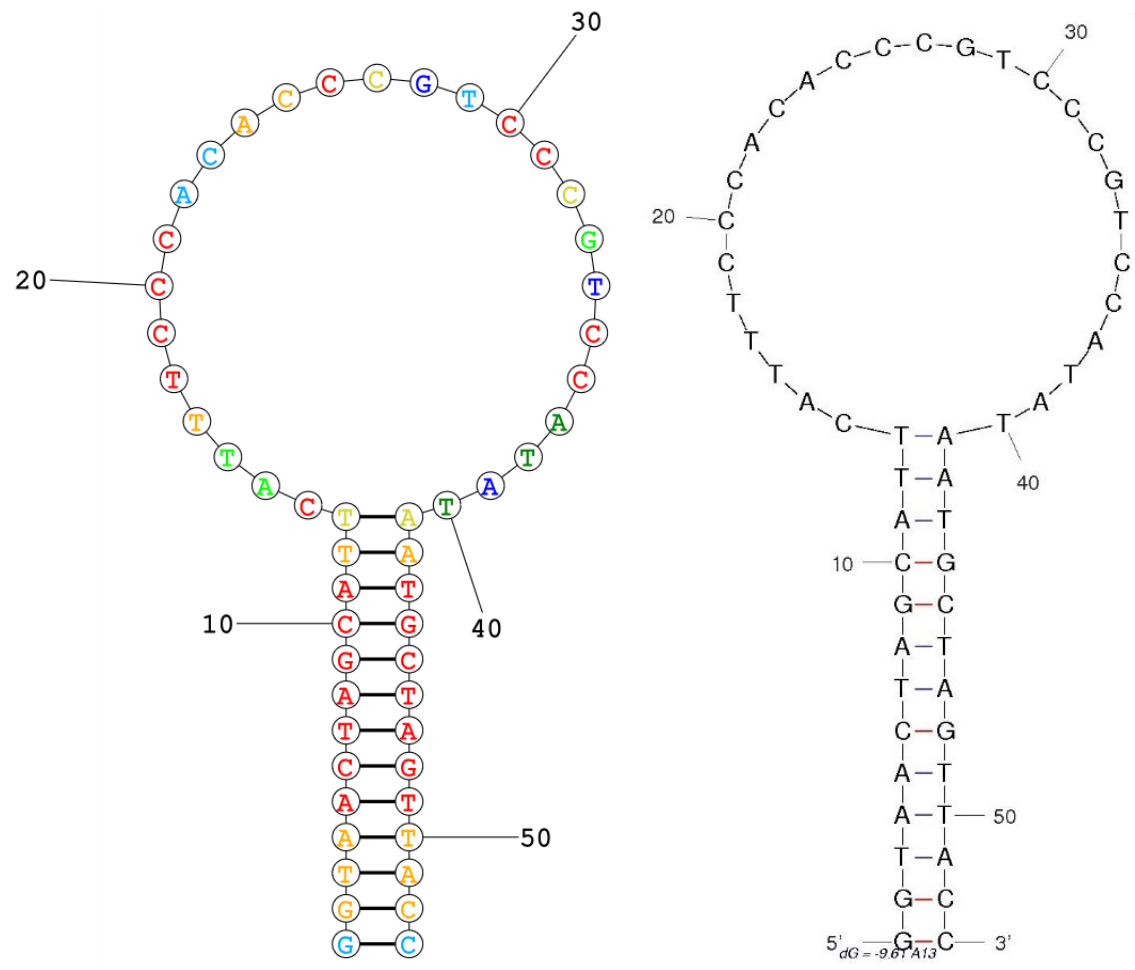

Figure 21 - Most probable secondary structure for the DNA sequence of the aptamer beacon with 13 complementary bases using RNAstructure (left) and Mfold (right) software (Reuter and Matthews, 2010; Zuker, 2003).
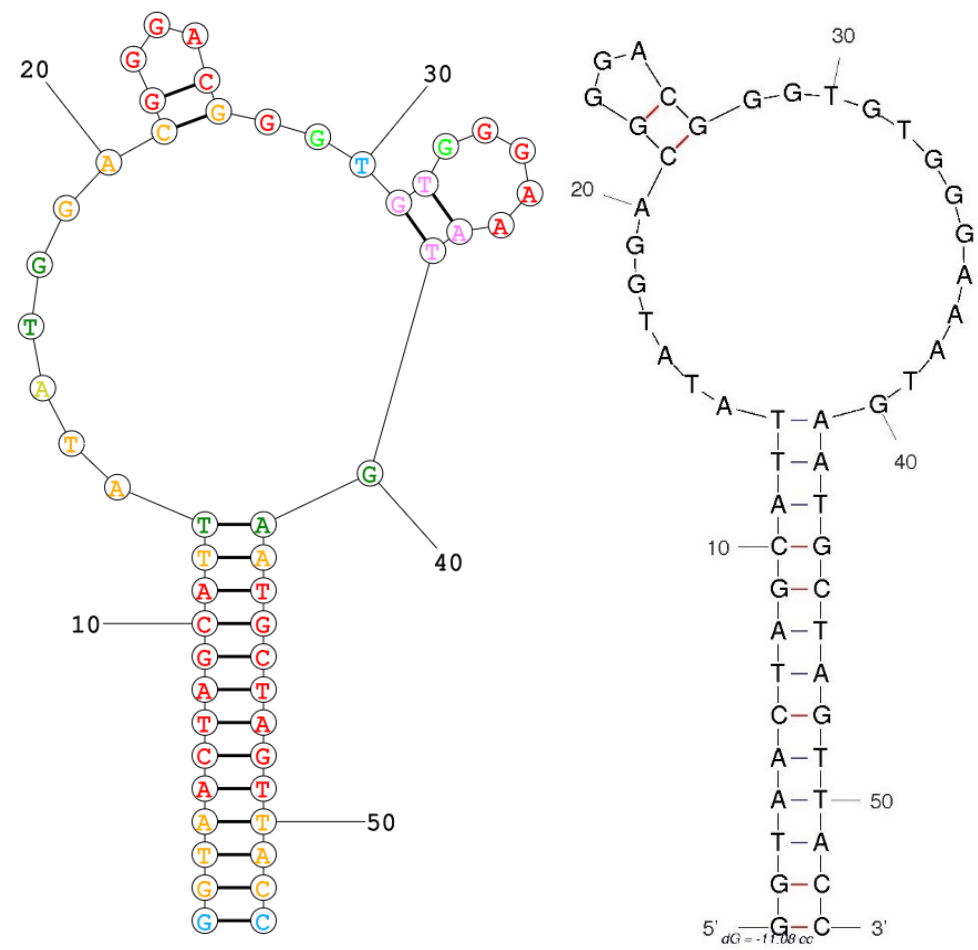

Figure 22 - Most probable secondary structure for complete complement DNA (CC) using RNAstructure (left) and Mfold (right) software (Reuter and Matthews, 2010; Zuker, 2003). 


\subsubsection{Linear Range of Fluorescence}

CC, FLX, and FLQ samples were synthesized and confirmed pure by mass

spectrometry (Appendix B: CCFL, FL3, and FLQ5). Solutions of FLX were prepared to observe the linear range of fluorescence detection (Figure 23). Samples above $0.05 \mathrm{nM}$ followed a linear regression. Using blank buffer well readings the LOD was determined to be $0.08 \pm 0.03 \mathrm{nM}$ and LOQ was determined to be $0.22 \pm 0.09 \mathrm{nM}$.

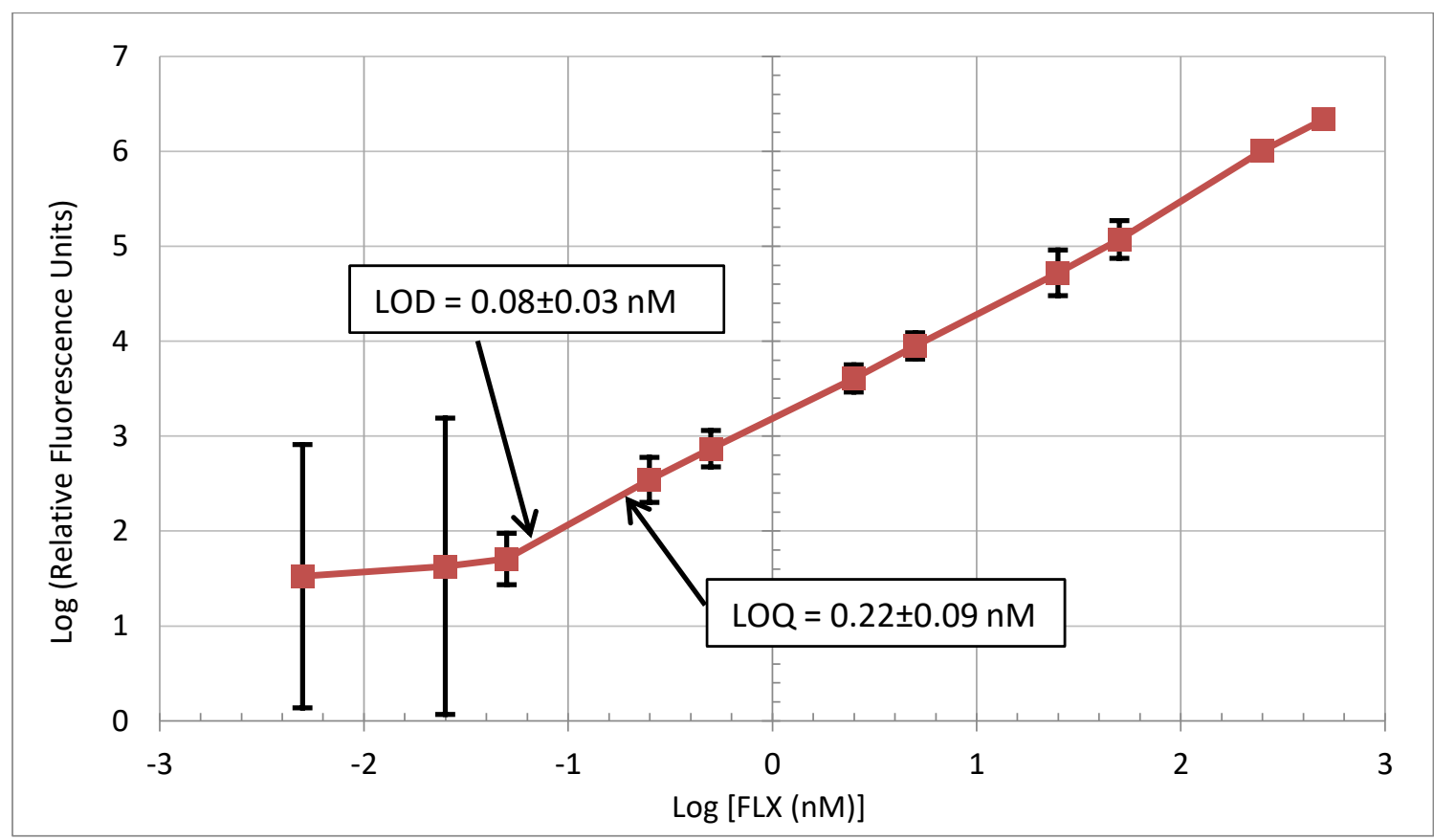

Figure 23 - Fluorescent control (FLX) calibration curve in TES buffer. Data points are the average of 9 wells over 3 replicates. Error bars represent 95\% Cl. Ex. $495 \pm 10 \mathrm{~nm}$, Em. $528 \pm 20 \mathrm{~nm}$.

\subsubsection{Potential Fluorescence}

To determine the potential inducible fluorescent signal FLQ was mixed with CC and heated for reannealing (Figure 24). FLX was used as a control of maximum fluorescence without quencher. At $0.25 \mathrm{nM} F L Q$ and FLQ+CC had less fluorescence than FLX. As concentration of FLQ+CC increased, fluorescence increased until it reached the 
signal of FLX at $2.5 \mathrm{nM}$. As concentration increased the difference between FLQ alone and FLX increased.

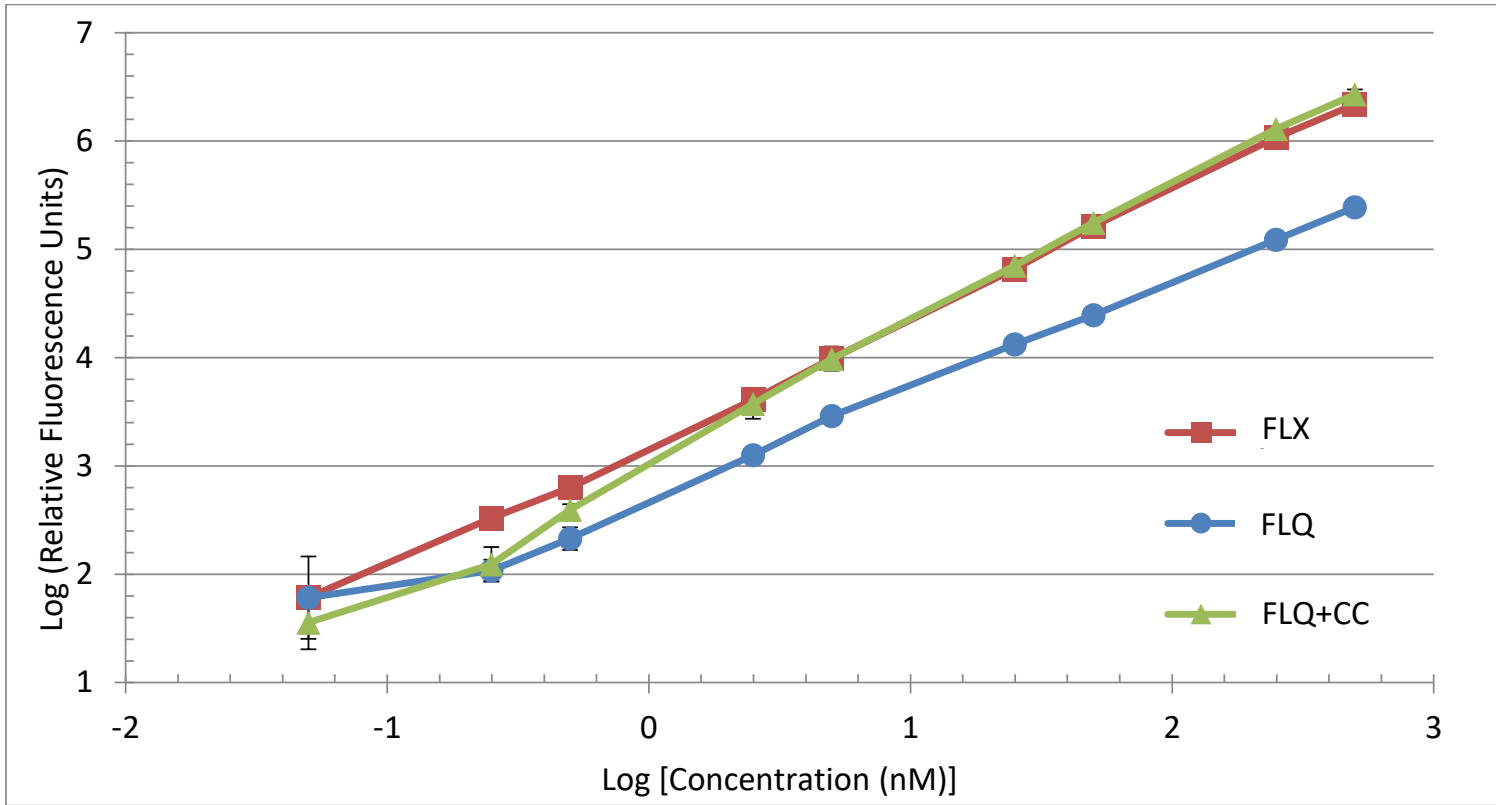

Figure 24 -Comparison of the fluorescence of fluorescein / dabcyl labeled aptamer beacon (FLQ) in the presence of equimolar complete complement DNA (CC) compared to fluorescein labeled aptamer (FLX) and FLQ alone in TES buffer. Data points are the average of 9 wells over 3 replicates. Error bars represent 95\% Cl. Ex. $495 \pm 10$ nm, Em. $528 \pm 20 \mathrm{~nm}$.

\subsubsection{Molecular Beacon Potential in TSB}

Fluorescence of the aptamer beacon in TES and TSB was determined at $10 \mathrm{nM}$ (Figure 25). FLX slowly decreased in fluorescence in TES and TSB. CC samples did not have significantly higher signal compared to the buffer alone while FLQ did. FLQ+CC in both cases increased signal and then stabilized. For TSB the fluorescence for FLQ+CC increased to being above FLX, while in TES it reached the same value. All TSB samples had much higher signal compared to TES samples. 


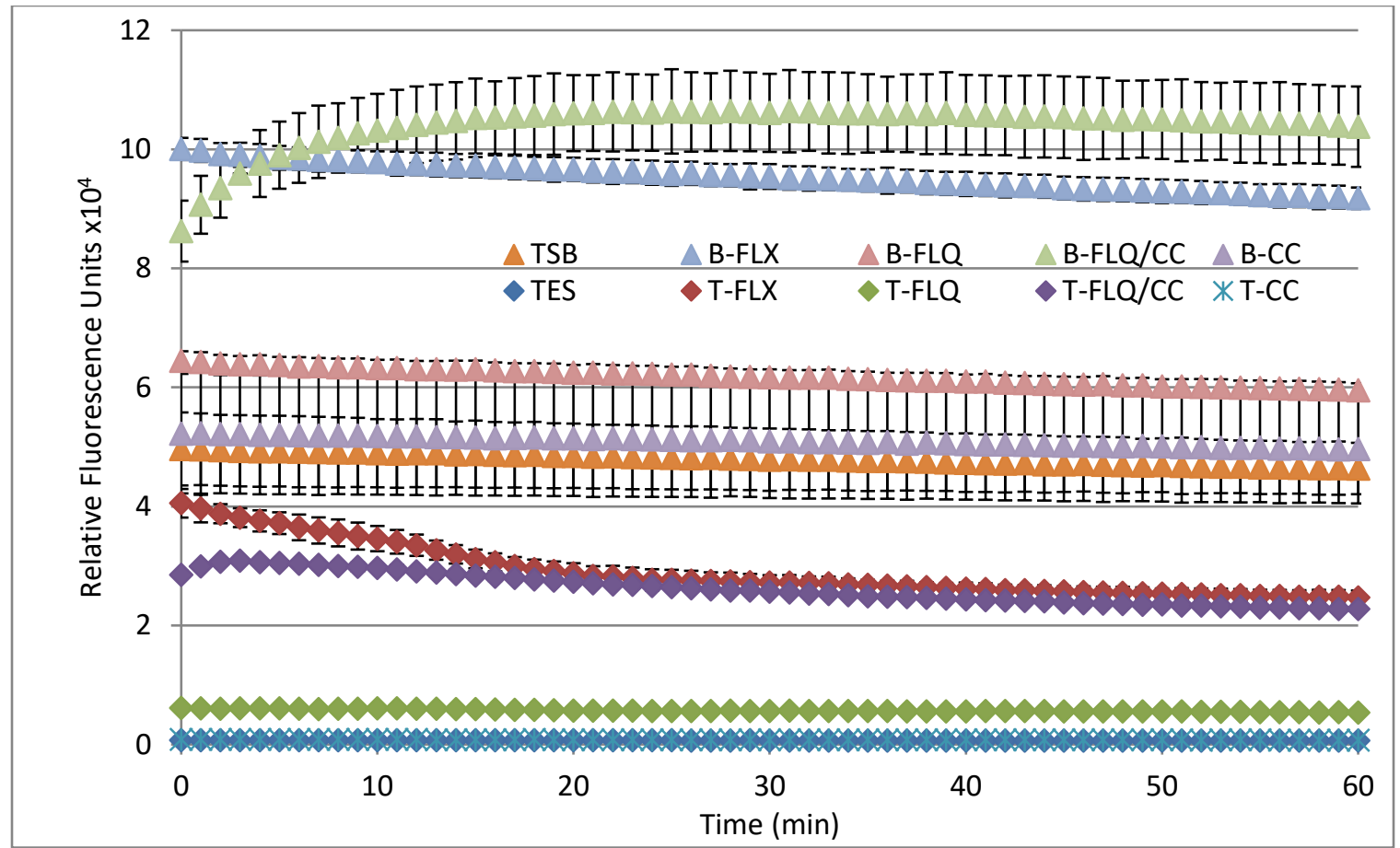

Figure 25 - Fluorescence of fluorescein / dabcyl labeled aptamer beacon (FLQ) induced by binding complete complement DNA (CC) (10 nM) in TES buffer and TSB media over time compared to fluorescein labeled aptamer (FLX). Data points represent the average of 3 samples, except for B-FLQ/CC (2 samples). Error bars represent SD. Ex. $495 \pm 10 \mathrm{~nm}, \mathrm{Em} .528 \pm 20 \mathrm{~nm}$.

\subsubsection{Fluorescence in the Presence of Toxin}

The fluorescence of FLQ as induced by VT was tested at $10 \mathrm{nM}$ (1:1 ratio) over time (Figure 26). Panel A shows the full scale with FLX having a much higher fluorescence compared to other samples. FLX signal decreased to about $37 \%$ after an hour. Panel B shows FLQ in buffer alone, with VT1a, or with VT2a. Over time FLQ and FLQ+VT2 decreased in fluorescence. FLQ+VT1 fluorescence decreased more slowly. FLQ, FLQ+VT2, and FLQ+VT1 fluorescence signal only decreased to 82, 86 and 94\%, respectively, over the 60 -minute incubation. After 70 minutes FLQ+VT1 was clearly distinct from FLQ alone. 

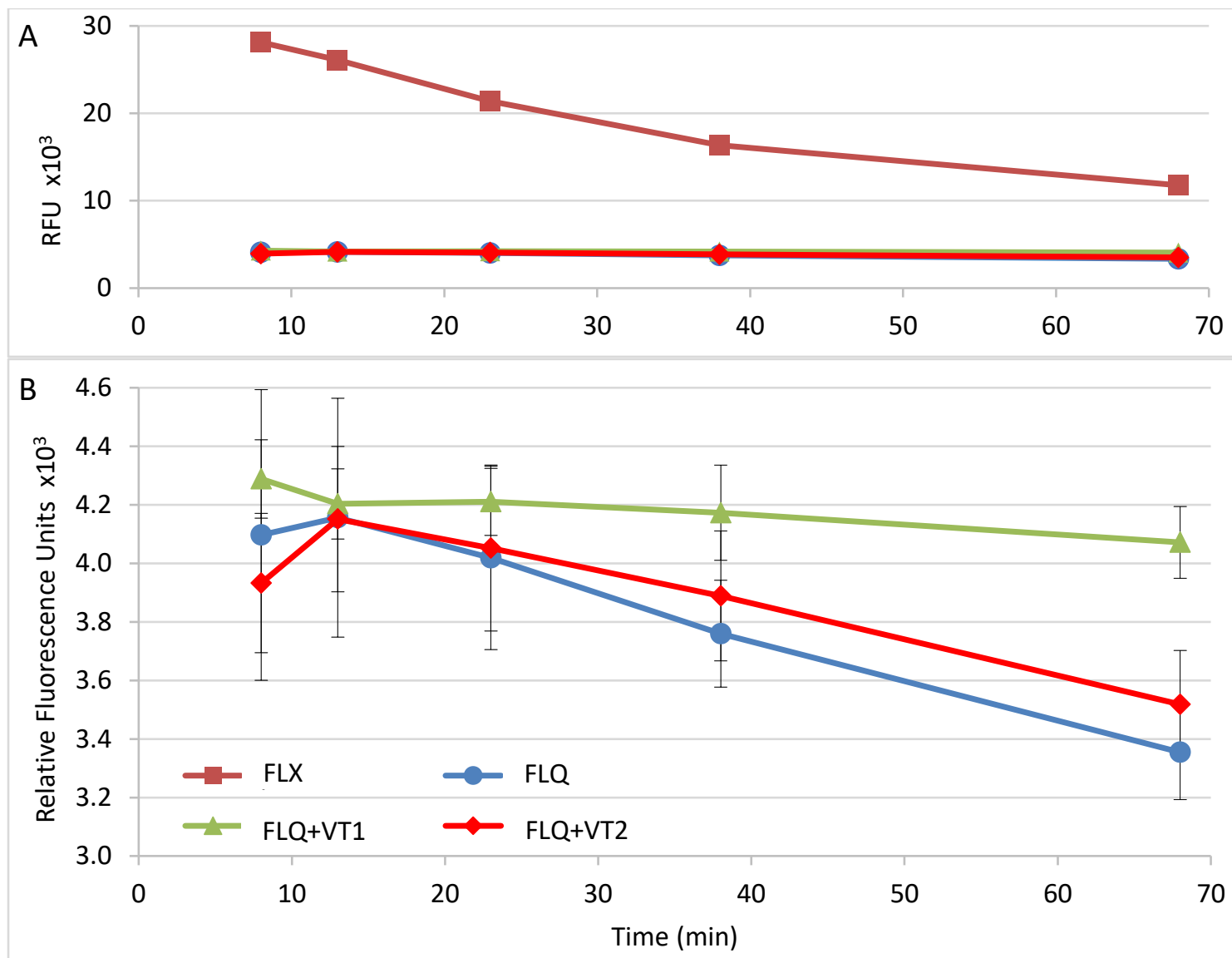

Figure 26 - Fluorescence of fluorescein / dabcyl labeled aptamer beacon (FLQ) (10 nM) with equimolar VT1a or VT2a over time in TES buffer. A) Fluorescence intensity of all samples; B) fluorescence intensity excluding fluorescein labeled aptamer (FLX). Data points are the average of 12 wells over 4 replicates, except for VT2 (9 over 3). Error bars represent 95\% Cl. Ex. $495 \pm 10$ $\mathrm{nm}, \mathrm{Em} .528 \pm 20 \mathrm{~nm}$.

\subsubsection{Limit of Detection with FLQ}

Having seen multiple times that VT1 caused a slower decrease in fluorescence signal for the beacon, the amount of toxin that could cause a difference was determined with $10 \mathrm{nM}$ of FLQ and decreasing concentrations of VT1 (Figure 27). As toxin concentration decreased the fluorescent signal decreased. The standard deviation of the solutions in the average of all four replicates overlapped heavily at all time points (Figure A-14). Replicates $1 \& 2$ (Figure A-14) and 3\&4 (not shown) were much closer in 
initial fluorescence values. Fluorescence values of solutions $1.25 \mathrm{nM}$ or higher after 70 minutes were significantly different from the $0 \mathrm{nM}$ solution with $10 \mathrm{nM}$ being significantly different by 25 minutes (Table A-1; $p=0.05$ ).

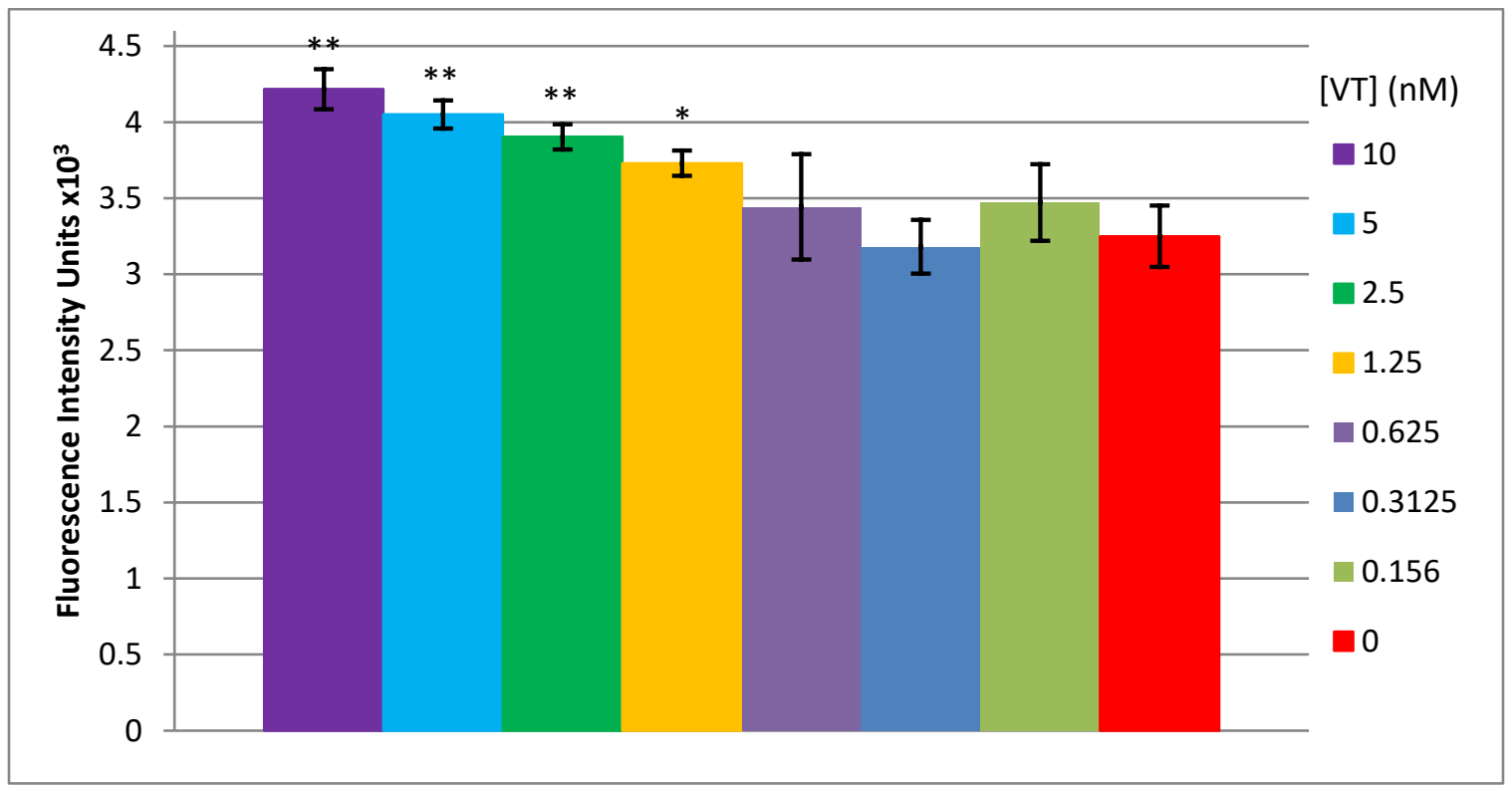

Figure 27 - Fluorescence of fluorescein / dabcyl labeled aptamer beacon (FLQ) (10 nM) after 70 minutes with varying VT1 concentrations in TES buffer. Fluorescein labeled aptamer (FLX) values $(10 \mathrm{nM})$ were around $17 \times 10^{3}$ RFUs. Data points represent the average of 6 wells from replicates 1 and 2. Error bars shown are SD. Ex. $495 \pm 10 \mathrm{~nm}, \mathrm{Em} .528 \pm 20 \mathrm{~nm}$. * and ** values significantly different from 0 nM VT $p=0.05$ and $p=0.01$, respectively.

FLQ was combined with varying concentrations of VT1 immediately before plating and reading (Figure 28). By eliminating time delays from plating the interaction for the first 10 minutes could be observed. The $0.63 \mathrm{nM}$ showed the general trend seen in the other samples: an increase of fluorescence followed by a steep decrease which leveled off into a stable slow decrease. As concentration increased the initial increase was seen less and stability was reached more quickly. Only a small portion of the decrease could be seen for $5 \mathrm{nM}$ and only the slow decrease was seen at $10 \mathrm{nM} V \mathrm{VT}$. The 5 and $2.5 \mathrm{nM}$ VT solutions appeared to stabilize at the same fluorescence level. 


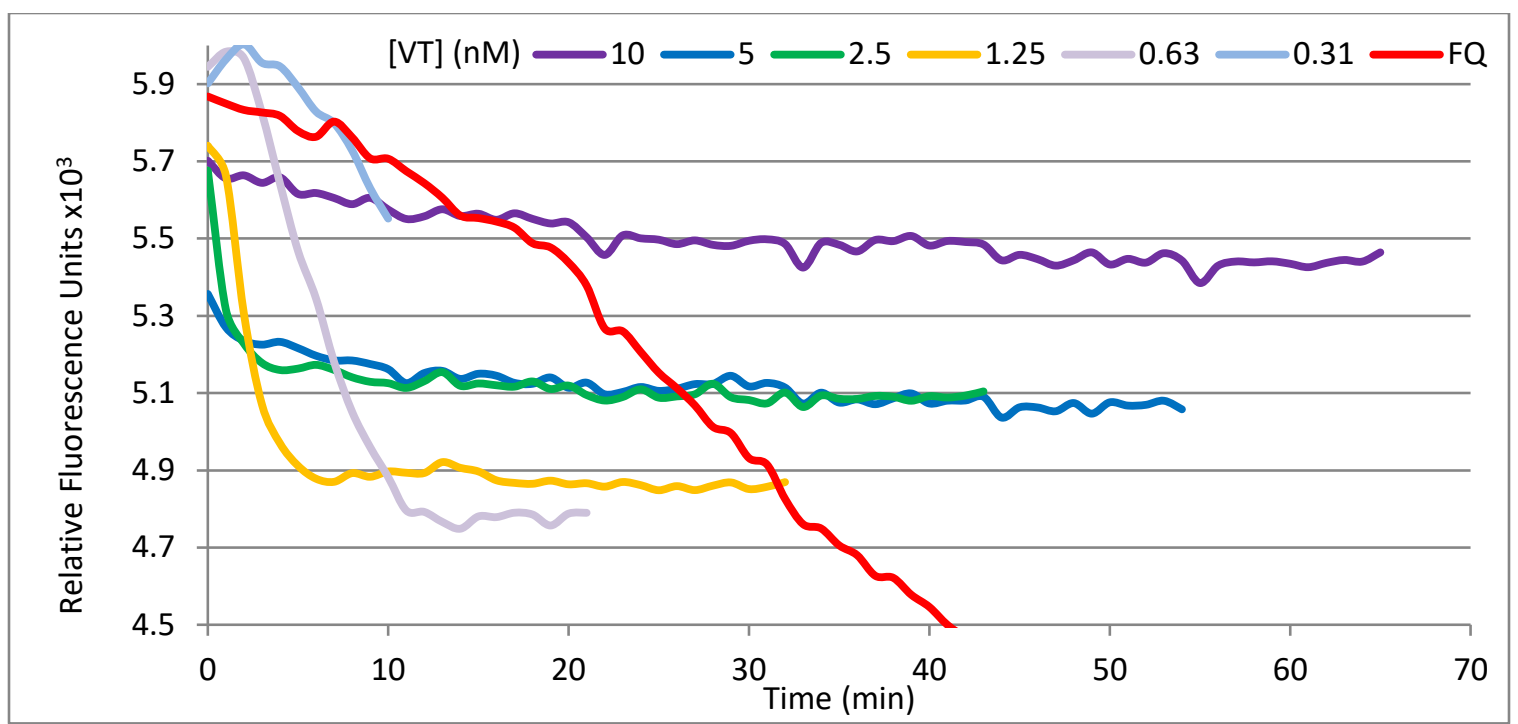

Figure 28 - Change in fluorescence signal of fluorescein / dabcyl labeled aptamer beacon (FLQ) in the presence of varying concentrations of VT1 mixed immediately before reading in TES buffer. Data points are the average of 9 samples over 3 trials. Ex. $495 \pm 10 \mathrm{~nm}, E \mathrm{~m} .528 \pm 20 \mathrm{~nm}$.

\subsubsection{Fluorescence in TSB}

The effect of toxin on fluorescence of FLQ was investigated in TSB since it would be more like the future application of the aptamer beacon (Figure 29). FLX had a much higher signal than FLQ samples. The inset shows that FLQ with VT1 increased slightly in signal, although error bars were overlapping. A decrease in fluorescence at the same rate over time was seen for both FLX and FLQ, while a slight increase was seen for FLQ+VT1 followed by a slow decrease.

The rate of fluorescent decay for free fluorescein (FI), FLX, and FLQ in dilutions of TSB was determined over an hour (Figure 30). In TSB the rate of decay was similar for all three. As dilution increased the rate of decay increased. FLX showed the fastest decay in signal, Fl an intermediate value, and FLQ with the slowest decay rate barely influenced by concentration of TSB. 


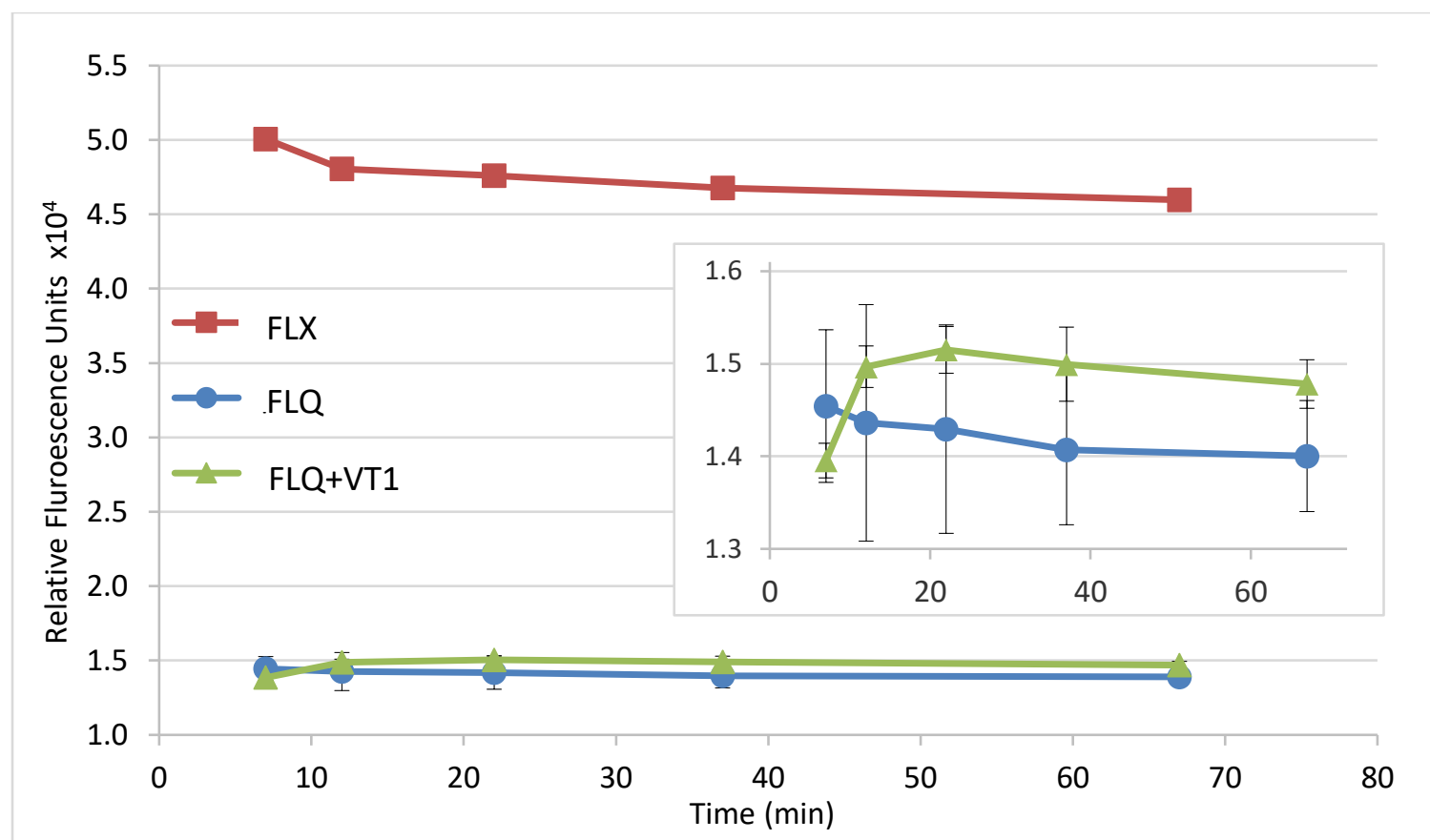

Figure 29 - Fluorescence of fluorescein / dabcyl labeled aptamer beacon (FLQ) (10nM) measured over time with equimolar VT1 in TSB media. Inset: closer view of FLQ and FLQ+VT1. Data points are the average of 6 wells over 2 replicates. Error bars represent $95 \% \mathrm{Cl}$. Ex. $495 \pm 10 \mathrm{~nm}, \mathrm{Em}$. $528 \pm 20 \mathrm{~nm}$.

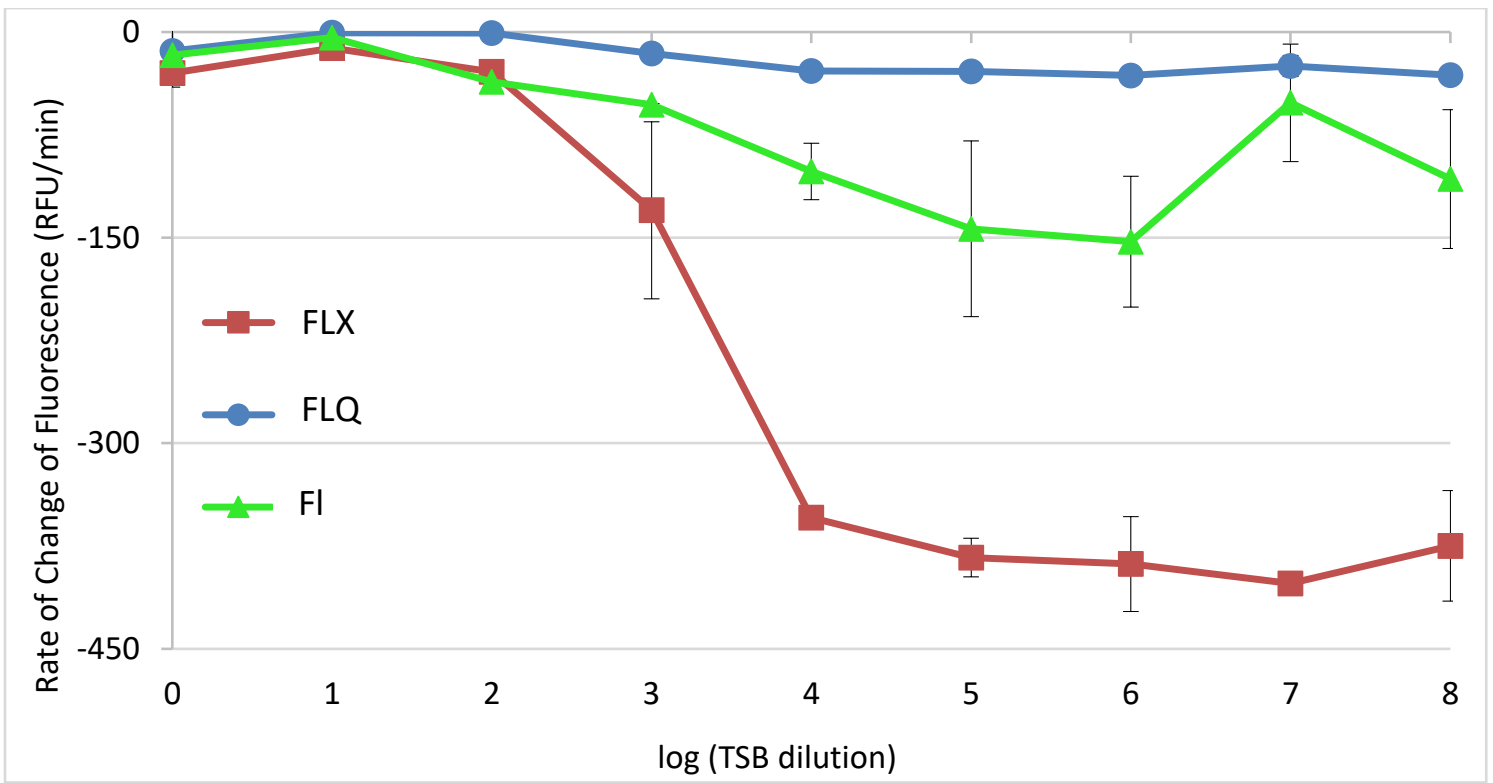

Figure 30 - Rate of fluorescence change of fluorescein labeled aptamer (FLX), fluorescein / dabcyl labeled aptamer beacon (FLQ), or free fluorescein (FI) at $10 \mathrm{nM}$ in TSB diluted with TES buffer over a 60-minute period. Data points are the average of 6 wells over 2 replicates. Error bars represent $95 \% \mathrm{Cl}$. Ex. $495 \pm 10 \mathrm{~nm}, \mathrm{Em} .528 \pm 20 \mathrm{~nm}$. 


\subsubsection{Fluorescent Signal Induced with Crude Toxin}

Crude VT1a (cVT1), crude VT2a, commercial VT1, commercial VT2, and Precision

Plus Protein ${ }^{\mathrm{TM}}$ Standards molecular weight ladder (BIO-RAD, CA, USA) were prepared and a PAGE gel was run (Figure 31). No bands were seen for the commercial toxins loaded. The CVT1 solution in lane 7 had a number of bands. VT was estimated to compose about $14 \%$ of the sample ( $8.7 \%$ A subunit; $5.3 \%$ B subunit). The total protein concentration was determined to be $265 \mu \mathrm{g} / \mathrm{mL}$ using a BCA assay. The amount of VT in the sample was about $37 \mu \mathrm{g} / \mathrm{mL}(23 \mu \mathrm{g} / \mathrm{mL}$ A subunit; $14 \mu \mathrm{g} / \mathrm{mL}$ B subunit) or $706 \mathrm{nM} \mathrm{A}$ subunit, $1.82 \mu \mathrm{M}$ B subunit, or 364 nM VT holotoxin with extra A subunits.

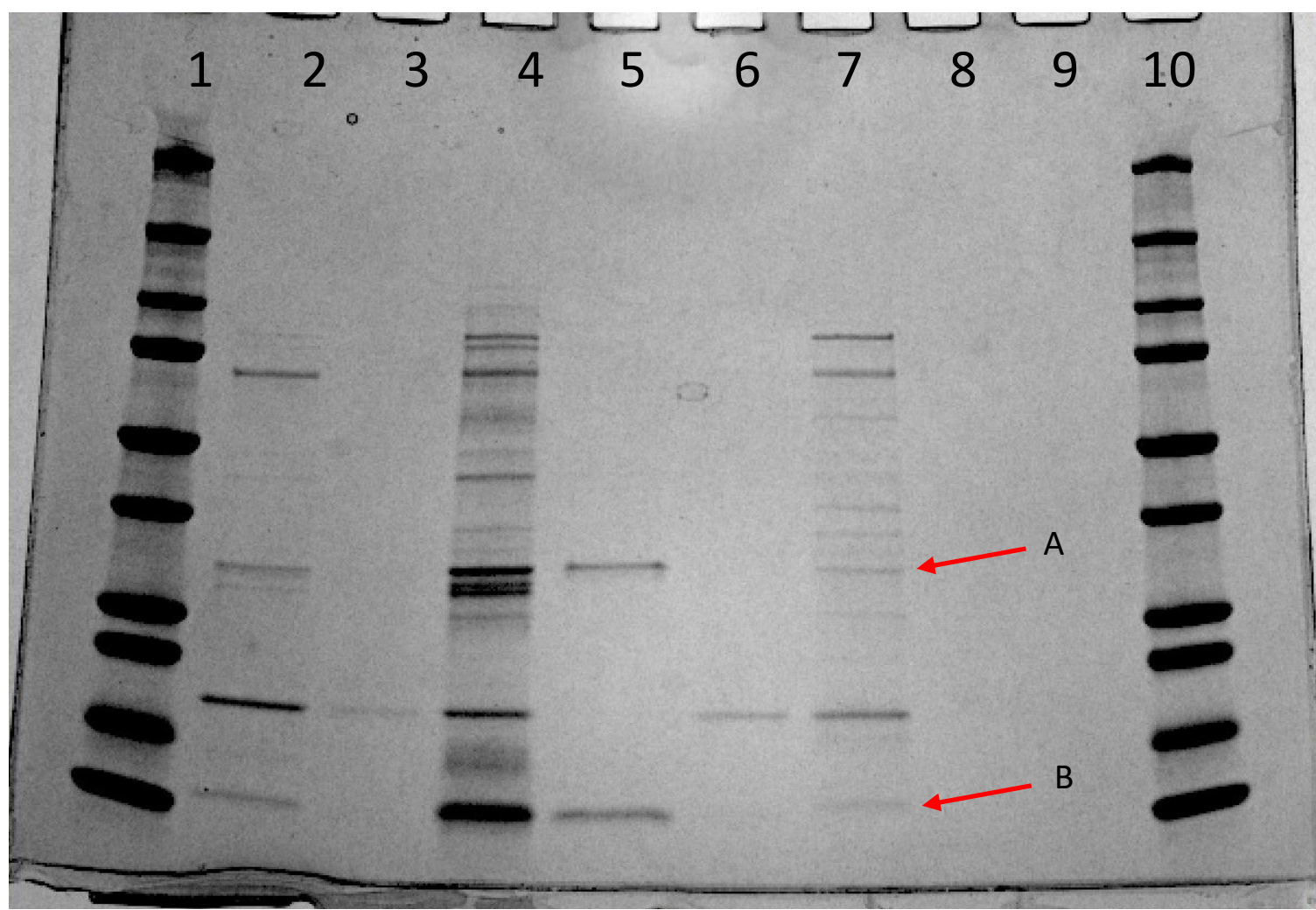

Figure 31 -SDS PAGE gel for crude VT1 preparation. Solutions loaded: 1) ladder; 2-6) crude VT2a solutions; 7) crude VT1a solution; 8) commercial VT1a; 9) commercial VT2a; and 10) ladder. A and $B$ subunits indicated for CVT1 by red arrows. 
The resulting fluorescence of FLQ and FLX with or without cVT1 at $2.5 \mathrm{nM}$ (VTL) and $6 \mathrm{nM}(\mathrm{VTH})$ of holotoxin was observed (Figure 32). These solutions had approximately 202 and 477 nM total protein, respectively. In Panel A the fluorescence of FLX was not decreased with addition of cVT. Higher concentration caused an increased stability of fluorescence. In Panel B the fluorescence of FLQ was decreased by addition of cVT, while also stabilizing. Higher concentration of cVT caused stabilized levels more rapidly at a slightly higher level.

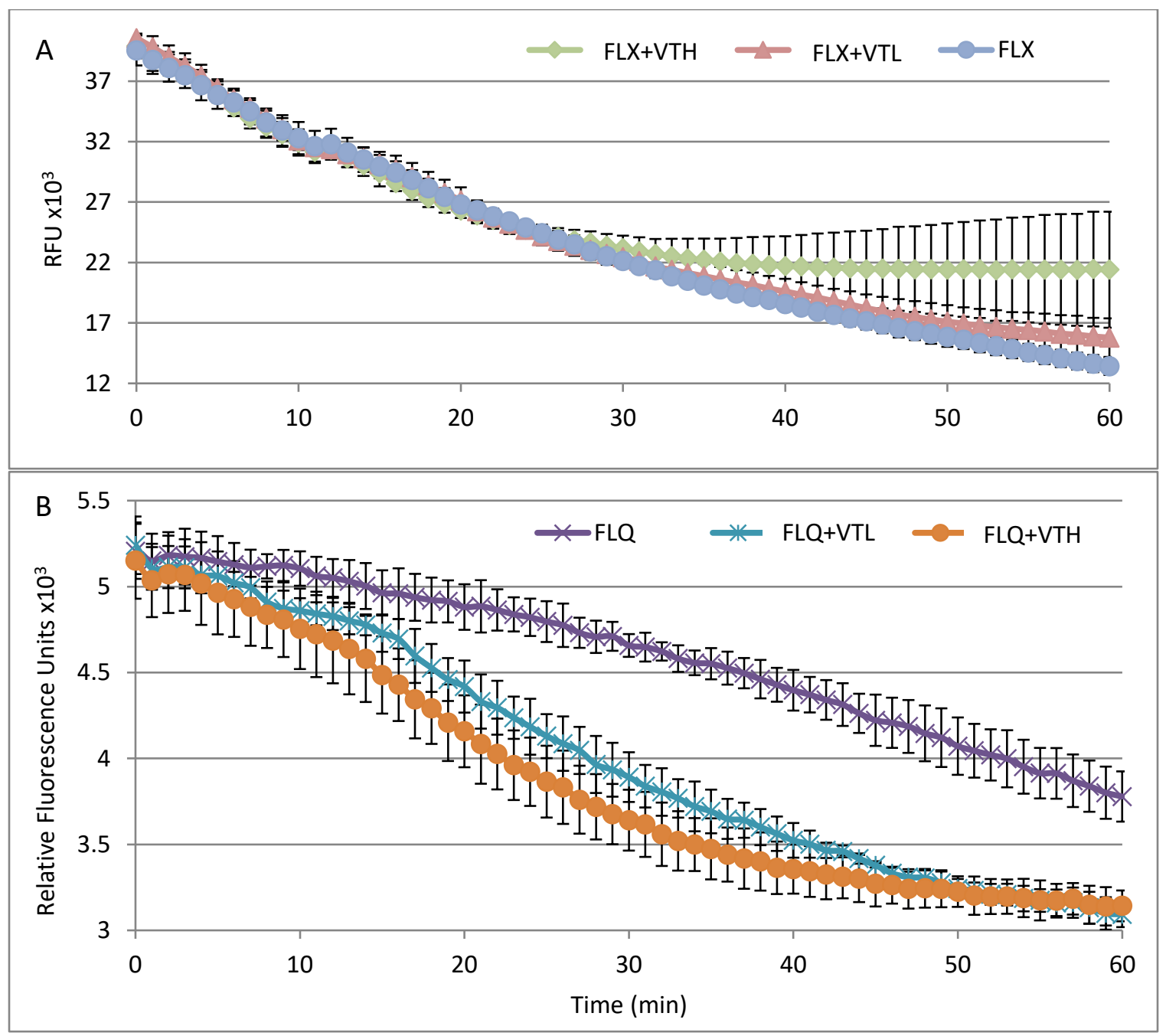

Figure 32 - Effect of crude VT1 on fluorescence of A) fluorescein labeled aptamer (FLX) (10 nM) and B) fluorescein / dabcyl labeled aptamer beacon (FLQ) (10 nM) at concentrations of $2.5 \mathrm{nM}$ (VTL) and 6 nM (VTH) of holotoxin over 60 minutes in TES buffer. Data points are the average of 9 samples over 3 replicates. Error bars are SD. Ex. $495 \pm 10 \mathrm{~nm}$, Em. $528 \pm 20 \mathrm{~nm}$. 


\subsubsection{Effect of Non-specific Protein Quenching}

Solutions of FLX and FLQ were mixed with VT1a, VT2a, bovine serum albumin (BSA), and TSB at $10 \mathrm{nM}$ in TES (Figure 33). In Panel A, VT1 caused immediate stabilization of the fluorescence, while VT2, BSA, and TSB caused no difference to the decreasing signal of FLX. After 60 minutes the signal of FLX had decreased to about $36 \%$ of the signal of FLX+VT1. In Panel B, VT1 caused an immediate drop in signal, remaining stable over time. VT2, BSA, and TSB caused a slight decrease in signal, but had no effect on the curve of signal loss.

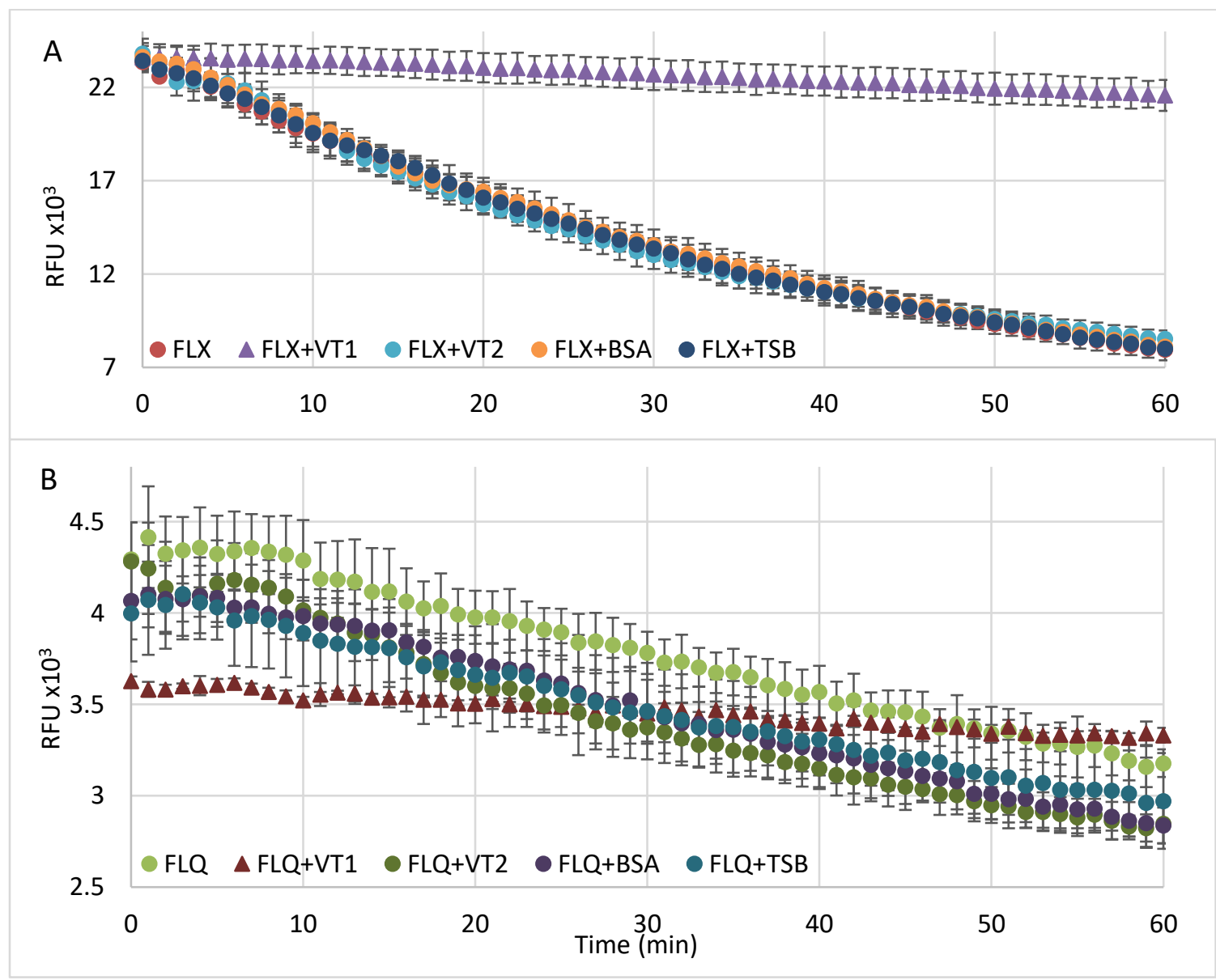

Figure 33 - Effect of protein on fluorescence of A) fluorescein labeled aptamer (FLX) (10 nM) and B) fluorescein / dabcyl labeled aptamer beacon (FLQ) (10 nM) in the presence of VT1, VT2, bovine serum albumin (BSA), or tryptone soya broth (TSB) (10 nM each) in TES buffer. Data points are the average of 3 samples, except for FLQ+VT1 (2 samples). Error bars are SD. Ex. $495 \pm 10 \mathrm{~nm}$, Em. $528 \pm 20 \mathrm{~nm}$. 


\subsection{Discussion}

\subsubsection{Aptamer Beacon Design and Synthesis}

FLQ was designed to be the mid-point between A10 and A15. As seen in Chapter 2.0, aptamer beacon with 10 bases of complement seemed to fluoresce too readily, resulting in larger background, while 15 bases seemed to be too selective. As such, a design with 13 bases was chosen. Fluorescein and dabcyl remained as the fluorophore and quencher. FLX was desirable as a fluorescent control because fluorescein and other fluorophores often have some degree of quenching by adjacent nucleotides (You et al., 2011). This factor would be controlled by having a fluorescent control with the same sequence. The aptamer beacon with 13 complementary bases had a secondary structure predicted as expected (Figure 21). As with A10 and A15 previously, the only structure predicted was in the complement region.

A complete complement was also desirable as complement only for the length of the hairpin did not fully open the aptamer beacon. To ensure a full conformation shift was attainable, a complement with all 53 bases was synthesized and named CC. Somewhat surprisingly there were some predicted secondary structures for CC in addition to the main hairpin (Figure 22). The small hairpin at base 21-27 between two pairs of C-G bonds was not predicted for the original sequence, although the reverse $\mathrm{G}$ and $\mathrm{C}$ nucleotides were present at bases $27-33$. This should not affect interaction with FLQ since hybridization between the complete sequence will have significantly higher affinity. The weaker predicted hairpin would cause even less potential competition. 


\subsubsection{Linear Range of Fluorescence}

A linear range, limit of detection (LOD), and limit of quantitation (LOQ) were determined for fluorescein labeled aptamer (FLX) (Figure 23). Concentrations between $0.05 \mathrm{nM}$ and $500 \mathrm{nM}$ fall within a linear dynamic range and would be acceptable for experimentation. Since the FLQ sequence was modified with a quencher its fluorescence will be less than that of FLX, working concentrations should be well above the LOQ.

\subsubsection{Potential Fluorescence}

When heated in the presence of $\mathrm{CC}$ there was an increase in fluorescence signal of FLQ (Figure 24). This fluorescent increase was due to hybridization with CC. The aptamer beacon could completely hybridize into a linear double-stranded DNA conformation, fully separating the fluorescein and dabcyl molecule. The two main ways quenching is accomplished, contact quenching and fluorescence resonance energy transfer (FRET), require distances of $<100 \AA$ (Marras et al., 2002). Completely opening the hairpin would prevent any quenching, resulting in the increased fluorescence signal observed.

Although this experiment was to observe the maximum potential fluorescent signal, in all likelihood the interaction with VT would not cause complete extension of the two ends of the DNA. Aptamer interaction with target molecules is dependent upon specific secondary and tertiary structures (Famulok and Mayer, 2011). The specific mechanism, binding location, or conformation between this aptamer and VT1 is not understood, but any additional structure could cause the fluorophore and quencher to 
be proximal enough to allow for some quenching. $100 \AA$ of separation is about 30 bases of distance, which is more than half the aptamer beacon's length, so there is a good possibility that it would quench to some degree even upon interaction with VT.

FLX and FLQ+CC showed very little difference in fluorescence potential in this experiment. There was, however, a concentration dependence for this signal to be completely observed. At concentrations below $2.5 \mathrm{nM}$ the increased fluorescence did not increase to match FLX. This was likely due to the kinetics of the reaction, with lower concentration making interaction between the $F L Q$ and $C C$ molecules less likely in the time given for the interaction. At higher concentrations FLX was a good simple positive control for the maximum potential fluorescence of FLQ interacting. At $10 \mathrm{nM}$ there was minimal difference between the two values. The data points found at $0.05 \mathrm{nM}$ were below the previously calculated LOQ and LOD. Analysis at that level would be unreliable.

Based on these observations, experimentation at $10 \mathrm{nM}$ was chosen to be sufficient for future experiments. The aptamer and toxin would be able to interact well within 30 minutes, should be high enough above LOD to avoid inaccuracy, and would be significant for practical use in the future since VTEC can be induced to produce VT at concentrations of $30 \mathrm{nM}$ or greater (de Sablet et al., 2008; Mühldorfer et al., 1996).

\subsubsection{Molecular Beacon Potential in TSB}

The maximum fluorescence signal of FLQ with CC without heating together was investigated in TES buffer and TSB media (Figure 25). TSB samples had a higher signal at all times. Likely, the much higher salt content in TES caused a decrease in signal as 
dabcyl more effectively quenched fluorescein (Dubertret et al., 2001). TSB has a low salt content and would thus allow a higher background signal. FLQ+CC had fluorescence signal that increased rapidly before stabilizing. In TES the fluorescence signal of FLQ+CC rose quickly to match the FLX signal, while in TSB it took some time and rose above FLX. This suggests that the $\mathrm{CC}$ interacts fully with the FLQ sequence as it reaches equilibrium, which makes sense as CC represents a maximal affinity target for the FLQ sequence. In TSB it appears to take longer to reach the final hybridized state. This was likely due to the additional peptides in the TSB solution making it more difficult to interact. However, whether TES or TSB, as in the case of CC, a target with sufficient affinity, causing a major conformational change, is expected to cause a major signal increase.

\subsubsection{Fluorescence in presence of toxin}

The effect on fluorescent signal in the presence of VT at $10 \mathrm{nM}$ was investigated (Figure 26). As at $50 \mathrm{nM}$ previously (Figure 17), VT1a caused stabilization of fluorescence signal, while VT2 did not greatly affect the signal. The most notable observation from this data was that fluorescence of all samples decreased throughout the course of the experiment. This overall decrease in fluorescence can be explained by photobleaching of the fluorescein molecules (Song et al., 1995). Photobleaching is a process by which fluorescent molecules become irreversibly modified, resulting in the inability to fluoresce. When the fluorophore is in an excited state it can react with other molecules in the environment or other fluorophore molecules. This occurs at a fixed probability depending on the molecule, excitation light intensity, the excited state lifetime, 
chemical stability, and the concentration of dye and other molecules in solution (Song et al., 1995). However, the FLX signal decreased at a higher rate than FLQ alone, or with VT, even though they were all at the same concentration. This was likely caused by quenching the fluorescein. When quenching occurs, the fluorophore leaves the excited state. Less time spent in excited state reduces the chances of photobleaching reactions (Selvin, 2000). As such, FLX with only some slight potential quenching from adjacent nucleotide bases, and other FLX molecules, was provided little opportunity for quenching. FLQ intrinsically has quenching due to the presence of dabcyl molecules held in close proximity. Additionally, the interaction with VT1 may contribute extra stability to the dabcyl quenching and add quenching from amino acid residues (Chen et al., 2010).

After about 50 minutes a difference in signal was observed between FLQ with or without VT1. With VT1 fluorescence remained higher over time. Potentially, part of the expected increase in signal cause by interaction with VT was negated by the photobleaching observed. However, the proximity of dabcyl and VT likely had a protective effect on photobleaching, so this was likely minimal. Compared to the previous trial with CC (Figure 24) there was no large increase in signal observed, so it was more likely that no major conformational change to the aptamer beacon occurred. This could be a result of a very poor interaction which was not strong enough to outcompete the 13-base complement sequence. More likely, the target binding interaction may not occur at the hairpin region of the sequence. In both cases fluorescein would remain quenched. 
VT2 did not stabilize the signal or cause a difference compared to FLQ samples alone in buffer. The difference between VT1 and VT2 samples indicated that there is a selective interaction with VT1. However, the minimal signal difference greatly hinders use of the aptamer beacon as it is currently designed. At the moment, difference between samples was only discernable due to the effect of photobleaching on the control solution.

\subsubsection{Limit of Detection with FLQ}

Using the reduction of photobleaching as a means of detecting VT1, a minimal concentration for VT1 that could cause a significant difference after an hour was investigated (Figure 27). The average of replicates $1 \& 2$ were presented since over the course of four replicates the initial fluorescence intensities were variable (Figure A-14). Replicates $1 \& 2$ or $3 \& 4$ showed significant differences after an hour between samples without VT and those of higher concentration (down to $1.25 \mathrm{nM}$ ) (Table A-1; $p=0.05$ ). Detection of VT could be accomplished by preparing a solution and measuring compared to a control without VT after 70 minutes, or longer to enhance the difference. This was unlikely to be able to quantify the presence of VT.

Generally, higher VT concentrations resulted in higher levels of fluorescence after 70 minutes. 5 and $2.5 \mathrm{nM}$ solutions, however, reached similar levels when time factors were removed (Figure 28). This may be due to an interplay of factors: higher toxin concentration could cause higher levels of induced fluorescence; higher concentrations could more quickly interact minimizing the effect of initial 
photobleaching; higher concentrations causing more protein quenching; and other potential factors.

The general interaction between toxin and the aptamer beacon appeared to follow a curve of an increase of fluorescence followed by a steep decrease which leveled off into a stable slow decrease. Higher concentrations reached equilibrium more quickly at higher levels of fluorescence (Figure 28). The increased speed of reaching equilibrium was likely due to the random nature of interaction in solution.

The peaks in fluorescence observed at 0.63 and $0.31 \mathrm{nM}$ provide strong evidence that there was an interaction between the beacon and the toxin that caused some conformational change, albeit potentially small or temporary. There was a sharp decrease in signal seen following the increase. This was seen at least somewhat in samples of $5 \mathrm{nM}$ and lower. It appeared that this drop occurred more and more quickly with higher concentrations and may even occur in the minute before reading for the 10 nM VT solution. The rate of decrease was even more rapid than the photobleaching observed with FLQ alone and occurred somewhat after the increase in fluorescence. This seems to suggest that a highly favourable interaction that results in decreased fluorescence was occurring after the initial interaction with VT. The overall rate of photobleaching of FLQ after the sharp drop was about the same in each of the solutions from 10-0.63 nM. This suggests that all of the aptamer beacon was interacting at each concentration. If less was interacting as concentration decreased, one would expect intermediate slopes with some decaying quickly and some slowly.

One potential explanation for the aptamer beacon interactions observed is that 
the stoichiometry of the interaction is greater than $1: 1$. VT is an $A B_{5}$ protein toxin. As such interaction with the B subunit would result in a 1:5 interaction between toxin and aptamer beacon. This would explain solutions as low as $2 \mathrm{nM}$ having the same reduced photobleaching rate. Potentially, with 3 binding sites in each B subunit for Gb3 (Ling et al., 1998) the interaction could be as high as $1: 15$ explaining as low as $0.66 \mathrm{nM}$ having the same rate as $10 \mathrm{nM}$. The stoichiometry of the interaction could be solved using crystallographic techniques, or more quickly with use of isothermal titration calorimetry. Increased stoichiometry, however, does not explain the rapid decrease in fluorescence units seen in each scenario.

A couple potential reasons for the drop in fluorescence relates to an enhanced binding of additional aptamer beacon to toxin facilitated by the free complement region. This region would add a high affinity component to the toxin. Potential options are presented in Figure 34. In the first scheme beacon binding causes increase in fluorescence. Following this, the complementary regions of separate toxin-beacon complexes interact forming a larger aggregate. The new interaction of complementary regions quenches the fluorescence, while allowing the stabilizing interaction with the toxin to remain or enhance quenching. In the second scheme additional aptamer beacons preferentially bind to the same toxin cooperatively. This could either be from new aptamer beacon in solution or from others already interacting. Aptamer beacon removed from other toxins no longer fluoresce, resulting in a decrease in fluorescence. The aggregate in scheme A may even be an intermediate complex for scheme B. Additionally, the interaction with complement and toxin may provide more optimal 
quenching than complement alone removing even some of the background

fluorescence. Notably, in these schemes there is always one aptamer beacon still fluorescing per toxin. Additional aptamer beacon per toxin may become less favourable after 3 or 4 allowing more toxin to fluoresce at higher VT concentration. This allows the decreasing fluorescent values seen in Figure 28. However, the exact method and stoichiometry of interaction between the aptamer beacon and toxin is unknown.

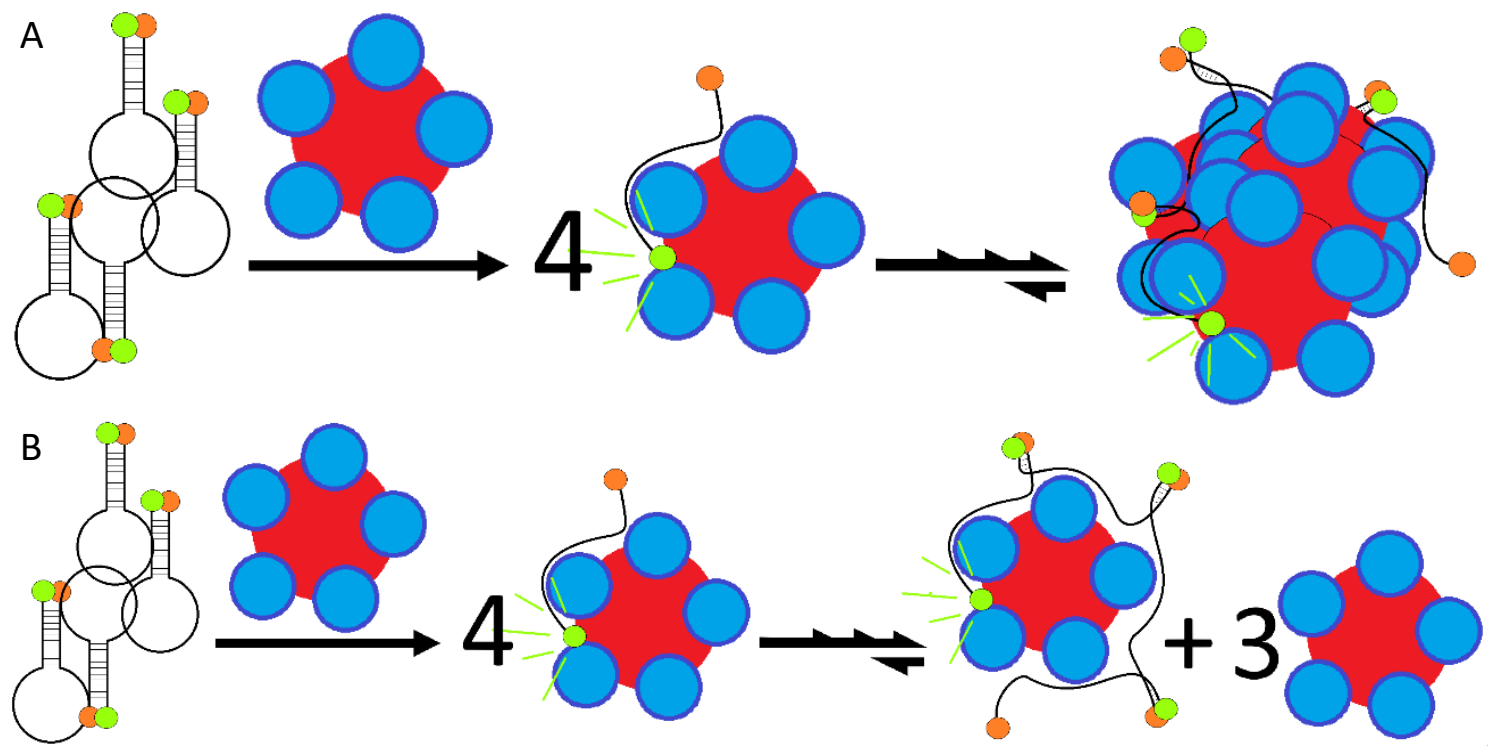

Figure 34 - Potential methods by which VT binds additional beacon cooperatively with the 5' complement region of the aptamer beacon. A) Each VT binds a separate beacon, followed by a shift towards toxin complexation. B) Beacon preferentially binds the same toxin, resulting in fewer toxins interacting with a higher number of beacons.

\subsubsection{Fluorescence in TSB}

Even in TSB the fluorescence of FLX was much higher than other samples (Figure 29). Furthermore, the increased signal of FLQ with toxin was minimal. Both of these observations continue to suggest a weak interaction between the beacon and toxin. Additionally, TSB has a lower salt concentration than TES which may cause the background to be higher than in previous experiments due to weaker quenching 
efficiency of dabcyl (Dubertret et al., 2001).

Seen in all TSB samples was a slow fluorescent signal decrease at the same rate. Photobleaching at a slower rate had previously been observed for the interaction of beacon with the toxin. TSB is a mixture of assorted peptides and non-specific interaction may occur with the aptamer beacon, causing a degree of protein-based quenching. With amino acids such as tryptophan able to absorb fluorescence, fluorescein spends less time in the excited state, and thus less time in a state vulnerable to photobleaching (Selvin, 2000). However, even if the assorted peptides were not being held together by interaction the way VT appears to be, the very high concentration ( $4500 \mu \mathrm{g} / \mathrm{mL})$ may facilitate this overall stabilization regardless.

To determine the effect of TSB on the rate of photobleaching a range of dilutions was prepared (Figure 30). At dilutions of 1-100x there was little effect and fluorescein was effectively stabilized with little decrease over 60 minutes. At dilutions of $10^{4}$ or greater there was little stabilizing observed. The rate of photobleaching for FLX increased the most while fluorescein alone only increased somewhat. FLQ remains with the lowest rate of photobleaching. It is unclear why fluorescein signal decreased more rapidly as part of FLX than alone in solution. Fluorescein is known to have fluorescence decreased depending on the nucleotide base it is attached to, especially guanine (You et al., 2011), which it is attached to in this aptamer beacon. This should add a quenching mechanism to FLX, but this was not observed. 


\subsubsection{Effect of Crude Toxin Prep}

The effect of mixing FLX (10 nM) or FLQ (10 nM) with a crude VT1 solution

(Figure 31) at $2.5 \mathrm{nM}$ and $6 \mathrm{nM}$ of holotoxin caused a stabilizing effect (Figure 32). As seen previously (Figure 27) increasing amounts of toxin reduced fluorescent decay. This was observed when combined with FLX where interaction with VT stabilized fluorescence. Addition of high concentrations of various peptides also has a strongly stabilizing effect on FLX (Figure 30). The other proteins in solution, at concentrations of 200 and $471 \mathrm{nM}$, would also result in some stabilization. The effect on FLQ resulted in curves similar to previous trials in buffer, but appeared to take longer to reach the final equilibrium. Previously FLQ with VT1 would have fluorescence decreased rapidly at first and then stabilized while FLQ alone decayed over time below that level. In this case it seemed that initial drop took nearly 60 minutes to reach the stable level and that the fluorescence level of FLQ would cross below if readings continued to be taken. The extra protein in the solution were likely making it more difficult for the aptamer to interact with the toxin. Additionally, general protein quenching likely lowered the fluorescence making it take longer for the FLQ sample to reach lower levels. The FLQ sample was not controlled with any sort of random protein in solution, so it was somewhat difficult to determine what would happen in that case. However, as it is now, the aptamer beacon is not useful in a filtered solution after 60 minutes. Potentially after 2 hours the stabilized value of FLQ with the toxin would remain higher than FLQ alone, however, if a protein control was introduced the stability added would likely decrease the rate of photobleaching making a difference less discernable. 


\subsubsection{Effect of Non-specific Protein Quenching}

Aptamers are generally selected to have a high specificity and affinity for their target molecule. The specificity of the VT1 aptamer (10 nM) in TES was tested with VT1a, VT2a, BSA, and a general amino acid solution of TSB (10 nM protein for each) (Figure 33). For FLX, only VT1 caused any significant change to the signal of FLX. Upon measuring fluorescence, the signal was stabilized. This suggests a strong interaction that quickly bound. Additionally, VT2 did not show any stabilization indicating that the aptamer sequence was selective for VT1. Previously stabilization was observed for FLX in undiluted TSB. By BCA assay TSB was determined to contain about $4468 \mu \mathrm{g} / \mathrm{mL}$ of protein $(\sim 67 \mu \mathrm{M})$. The effects of stability observed were likely due to the high concentration rather than a meaningful non-specific interaction. Panel B showed the same pattern observed for FLQ's interaction with VT1, a stabilized signal at a lower initial value. This again suggests that the interaction in some way helps facilitate quenching by dabcyl with some potential protein-based quenching. This was seen for the other 3 solutions which were not seen to change the rate of signal decrease, but were slightly lower in value. The VT aptamer sequence appeared to be fairly selective for VT1, although no enhanced signal was observed for VT1. Additionally, the signal with FLX did not decrease suddenly as in FLQ. As such, FLX would not be useful as a turn-off sensor. 


\subsection{Conclusions / Future Directions}

Although a VT aptamer beacon would be useful in making the detection of VTEC

a faster and simpler process, the VT aptamer beacon, as it is now, is unable to meaningfully accomplish that goal. The beacon was shown to be able to produce a full signal in both TES and TSB when complete complement DNA was added, but VT produced negligible signal. The interaction was, however, specific for VT1. As such it is highly likely that the binding interaction does not cause a major conformational change, resulting in minimal signal induction. This could be due to minimal interaction with the target, a binding structure that does not cause a major conformational shift, or potentially binding is occurring in the loop region of the beacon. Characterization of the sequence to identify the bases involved in VT interaction could assist in better aptamer beacon designed for VT1 without a new selection. Use of isothermal titration calorimetry or other methods could be used to determine the affinity of this interaction as well as the stoichiometry of the interaction (Velázquez-Campoy et al., 2004) as those are still gaps in the knowledge.

The interaction of VT1 with aptamer beacon caused stabilization of fluorescein photobleaching. This was exploited and significant difference in fluorescence was observed even down to $1.25 \mathrm{nM}$ in buffer after 70 minutes. There was some evidence that a signal was produced in the early minutes of interaction, however, this was quickly removed as the system approaches an equilibrium state, likely as a higher order complex of VT and aptamer beacon. In TSB media, no significant change was observed as the amino acids in the media stabilize all samples. Crude VT samples similarly did not 
cause a signal enhancement. Since the signal in buffer was not a sustained increase the effectiveness of the beacon is minimal. Likely the sensitivity of fluorescent measurements is why this interaction was even detectable. Addition of complexity to the system through impure VT or various peptides in general broth media reduced signal even further to insignificance.

A new aptamer beacon for VT should be developed with all the necessary components to be effective. The sequence would ideally target all VT, or VT2 as it is a higher risk factor. Selection could be performed in growth media with nuclease resistance modifications and selective pressure for structure switching to enhance the final desired application. The specificity, affinity, and bases involved in target binding of the aptamer should be determined before modification into an aptamer beacon. High specificity would be required for use in complex media. A fluorophore / quencher pair more stable, with lower background fluorescence, and suitable for use in media would be desirable. 


\subsection{Comparing Fluorophore / Quencher Pairs}

\subsection{Introduction}

\subsubsection{Fluorophore / Quencher Pairs}

Use of fluorescent molecules in detection allows for great sensitivity in sensing systems. However, as seen in initial testing with fluorescein and dabcyl there was minimal signal increase in the presence of VT. The fluorescence of fluorescein was difficult to observe on TSA plates. Additionally, the fluorescent signal rapidly photobleaches. Another pair of fluorophore and quencher molecules may solve these problems for future aptamer beacon use.

There are now a wide range of fluorescent dyes that are commercially available. These dyes cover a very wide range of the visible wavelength and can even be tuned to the specific wavelengths desired. Similarly, there are a number of molecules which can be used effectively as quenchers of those fluorophores through direct contact (static quenching) or transfer of energy over a small distance (dynamic quenching) (You et al., 2011). As such, pairs can be selected for their use and optimized for the conditions.

\subsubsection{Selecting New Pairs}

The primary problems observed with fluorescein and dabcyl labeled aptamer beacon were: strong background signal; weak increase in signal; and poor contrast on TSA plates. To overcome these problems two new fluorophore / quencher pairs were chosen for testing: Texas Red / Black Hole Quencher 2 (BHQ2) and Cyanine 5 / Black Hole Quencher 3 (BHQ3) (Table 6). 
Dabcyl is known as a universal quencher, however, its absorption is strongest from 380-530. Fluorescein's peak emission (522 nm) is at the edge of dabcyl's absorption spectra. Texas Red's and Cyanine 5's peak emissions (615 and $662 \mathrm{~nm}$ ) are near the peak absorption of BHQ2 and BHQ3, respectively (579 and $672 \mathrm{~nm}$ ). This should allow for more efficient quenching to minimize background signal.

To increase the contrast on TSA plates fluorophores with peak emission wavelengths closer to red were considered. Cyanine 5 was a readily available fluorophore with a much higher wavelength than fluorescein, although it still has some instability to temperature or neighbouring nucleotide bases. Texas Red appeared to be more stable than fluorescein and had a wavelength intermediate to fluorescein and Cy5. Texas Red was more expensive and more difficult to synthesize; however, the stability of the molecule was the deciding factor for choosing Texas Red.

\subsection{Chapter Objectives}

Fluorescein was found to perform poorly as a fluorophore as a component of the VT aptamer beacon. Additionally, fluorescein had poor contrast on TSA agar. To enhance the contrast and augment signaling from the beacon system two other fluorophore / quencher pairs were tested.

\subsection{Materials and Methods}

\subsubsection{Buffer}

TES (tris-EDTA high salt): $10 \mathrm{mM}$ tris, $0.5 \mathrm{mM}$ EDTA, $0.5 \mathrm{M} \mathrm{NaCl}, 0.5 \mathrm{M} \mathrm{KCl}$. pH 7.4 (adjusted with $\mathrm{HCl}$ and $\mathrm{NaOH}$ ). 
Table 6 - Characteristics of fluorophores and quenchers compared in this study

\begin{tabular}{|c|c|c|c|c|}
\hline Fluorophore & $\begin{array}{l}\text { Excitation / } \\
\text { Emission } \\
\text { Peak }(\mathrm{nm})^{*}\end{array}$ & Fluorophore Stability Effects & Quencher & $\begin{array}{l}\text { Absorption } \\
\text { Spectra; } \\
\text { Peak }(\mathrm{nm})^{*}\end{array}$ \\
\hline Fluorescein & $494 / 522$ & $\begin{array}{l}\text { - Extinction coefficient temperature- } \\
\text { dependent } \\
\text { - Salt-dependent loss of signal above } \\
40{ }^{\circ} \mathrm{C} \text { in low salinity } \\
\text { - Nonlinear change of signal }(+25 \text { to - } \\
11 \%) \text { as heated to } 90{ }^{\circ} \mathrm{C}^{\mathrm{a}} \\
\text { - Strong loss of signal in low } \mathrm{pH}^{\mathrm{a}} \\
\text { - Variable quenching by } \\
\text { neighbouring } \mathrm{G} \text { residues }\end{array}$ & Dabcyl & $\begin{array}{l}380-530 \\
453\end{array}$ \\
\hline Texas Red & $599 / 615$ & $\begin{array}{l}-<12 \% \text { loss after two cooling and } \\
\text { heating cycles } \\
\text { - Linear loss of } 14 \% \text { signal as heated } \\
\text { to } 90{ }^{\circ} \mathrm{C}^{\mathrm{a}} \\
\text { - }<10 \% \text { loss between } 6.5 \text { to } 7.8 \mathrm{pH}^{\mathrm{a}} \\
\text { - salt-dependent loss of signal; less } \\
\text { at higher salinity } \\
\text { - insensitive to environment }\end{array}$ & $\mathrm{BHQ2}$ & $\begin{array}{l}550-650 \\
579\end{array}$ \\
\hline Cyanine 5 & $646 / 662$ & $\begin{array}{l}\text { - Nonlinear loss of } 83 \% \text { signal as } \\
\text { heated to } 90^{\circ} \mathrm{C}^{\mathrm{a}}\end{array}$ & BHQ3 & $\begin{array}{l}620-730 \\
672\end{array}$ \\
\hline
\end{tabular}

* Optical values quoted from glenres.com; BHQ - Black Hole Quencher

a You et al., 2011. ' Liu et al., 2005.

\subsubsection{DNA Samples}

CC, FLX, and FLQ sequences prepared for Chapter 3.0 were used in this chapter.

Additionally, equivalent sequences labeled with Texas Red (Figure 35) or Cyanine 5 as

fluorophores, and Black Hole Quencher 2 (Figure 36), or Black Hole Quencher 3 (Figure

37) as quenchers were ordered. Sequences were as follows where the underlined

sections are complementary to form a hairpin (with the quenched version modified with the quencher in brackets):

TRX (TRQ): 5'-Texas Red-GGT AAC TAG CAT TCA TTT CCC ACA CCC GTC CCG TCC AT AT+ $\underline{A}$ ATG CTA GTT ACC-(BHQ2-)3'

CYX (CYQ): 5'-Cyanine 5-GGT AAC TAG CAT TCA TTT CCC ACA CCC GTC CCG TCC AT AT+트 ATG CTA GTT ACC-(BHQ3-)3' 
TRX was purchased from IDT (IA, USA) and TRQ, CYX, and CYQ, were all purchased from Sigma-Aldrich Corporation (MO, USA).

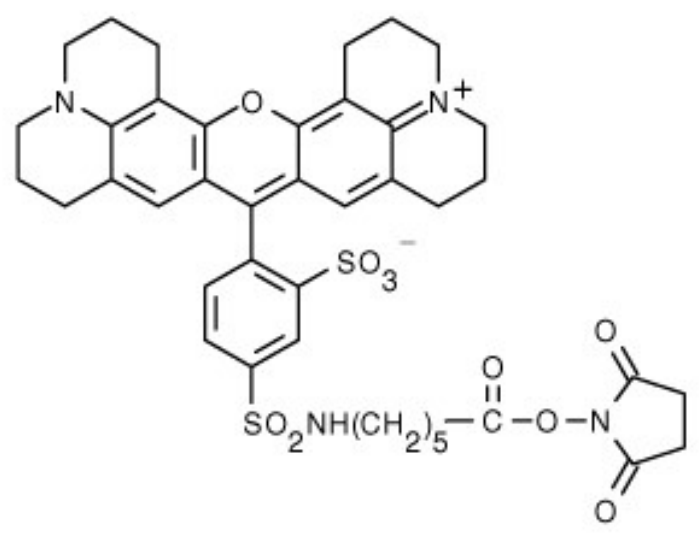<smiles></smiles>

Figure 35 - Chemical structures of left) Texas $\operatorname{Red}^{\circledR}-X$, succinimidyl ester; and right) Cy5 phosphoramidite, 1-[3-(4-monomethoxytrityloxy)propyl]-1'-[3-[(2-cyanoethyl)-( $\mathrm{N}, \mathrm{N}$-diisopropyl phosphoramidityl]propyl]-3,3,3',3'-tetramethylindodicarbocyanine chloride

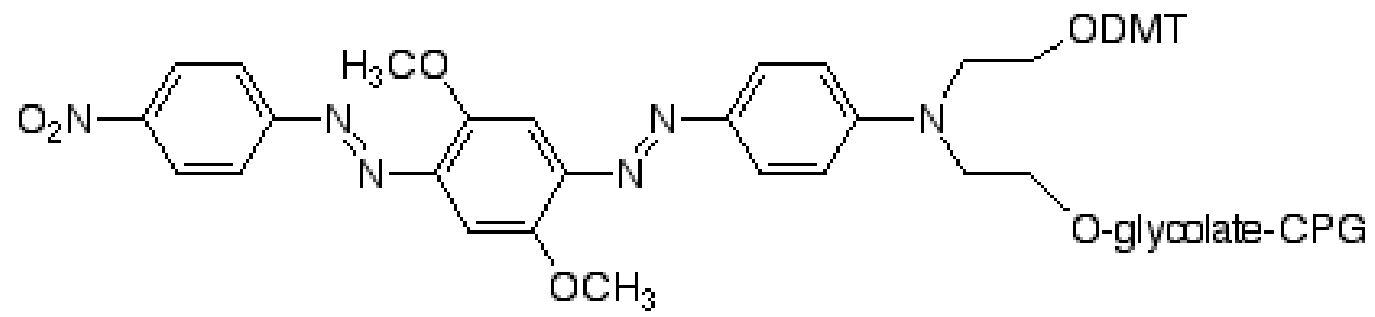

Figure 36 - Black Hole Quencher 2 CPG, (4'-(4-Nitro-phenyldiazo)-2'-methoxy-5'-methoxyazobenzene-4"-(N-ethyl-2-O-(4,4'-dimethoxytrityl))-N-ethyl-2-O-glycolate-CPG)

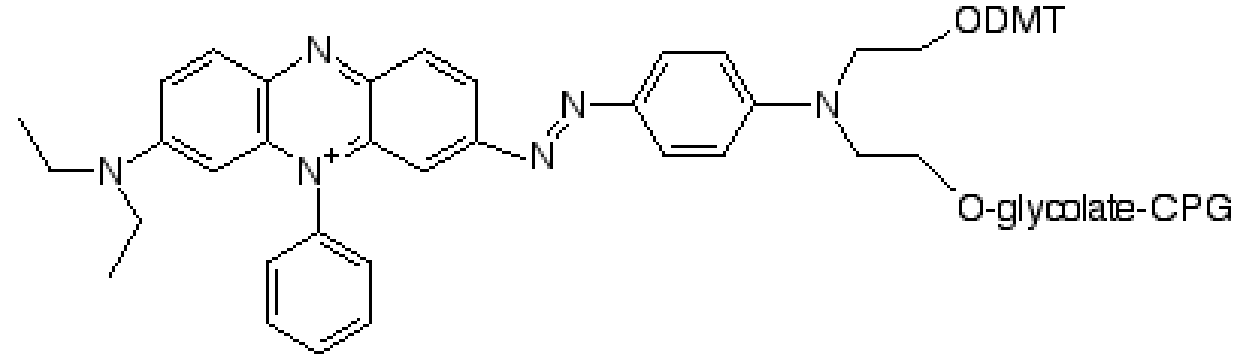

Figure 37 - Black Hole Quencher 3 CPG, (3-Diethylamino-5-phenylphenazium-7-diazobenzene-4"'(N-ethyl-2-O-(4,4'-dimethoxytrityl))-N-ethyl-2-O-glycolate-CPG)

\subsubsection{Fluorescent Measurements}

Fluorescence measurements of samples were performed using a Synergy 2 microplate reader (Biotek) with Gen5 software, in 96-well black microtiter plates (Nunc). 
A 50/50 mirror and a sensitivity setting of 90 was used throughout. Readings were taken with different optical filters sets for each fluorophore: fluorescein $495 \pm 10 \mathrm{~nm}$ for excitation, and $528 \pm 20 \mathrm{~nm}$ for emission; Texas Red $596 \pm 15 \mathrm{~nm}$ for excitation, and $680 \pm 30 \mathrm{~nm}$ for emission; and Cy5 $620 \pm 40 \mathrm{~nm}$ for excitation, and $680 \pm 30 \mathrm{~nm}$ for emission.

DNA samples were prepared by dilution to the desired concentration. Samples were heated at $90^{\circ} \mathrm{C}$ for 10 minutes and allowed to cool at room temperature for 30 minutes before plating or mixing with toxin. Samples were plated in $200 \mu \mathrm{L}$ aliquots on black 96-well plates for testing. All readings were taken at room temperature.

\subsubsection{Determination of Linear Fluorescent Range}

Samples of FLX, TRX, and CYX were prepared at concentrations of 0.0025 to 50 $\mathrm{nM}$ in TES buffer and plated in triplicate. Fluorescent readings were taken. Three replicates were performed on separate days. The LODs and LOQs were calculated.

\subsubsection{Determination of Potential Fluorescence}

Samples of FLX, FLQ, and FLQ+CC were prepared at concentrations of 0.025 to 50 nM in TES buffer and plated in triplicate. Fluorescent readings were taken. Three replicates were performed on separate days. This experiment was repeated for Texas Red and Cy5 versions of the beacons.

\subsubsection{Interaction with VT}

Samples of TRX and TRQ were prepared. TRQ samples were mixed with VT1, with VT2, or without VT at a 1:1 ratio with final concentrations of $50 \mathrm{nM}$ in TES buffer and 
plated in triplicate. VT in buffer was plated once per plate. Fluorescent readings were taken each minute for 60 minutes. Three replicates of the experiment were performed on separate days. This was repeated with Cy5 versions of the beacons.

\subsubsection{Comparison of Visible Limit of Detection}

Fluorescein (FI), Texas Red (TR), and NIR-641 (equivalent to Cy5, Figure 38) were purchased from Thermo-Fisher Scientific (MA, USA). Stock solutions of FI, TR, and Cy5 were prepared by dissolving in DMSO and dilution in deionized water. Solutions of each were prepared at $100,10,1,0.1$, and $0.01 \mu \mathrm{M}$. Fluorescence in solution was observed with a handheld UV lamp. Spotting of reagents was done on Tryptone Soya Agar (TSA) and $1.5 \%$ Agarose (15 g/L agarose). $10 \mu \mathrm{L}$ of FI and TR were spotted in quintuplicate at $40,30,20,10,5$, and $1 \mu \mathrm{M}$ on separate plates as well as being plated in triplicate on a plate adjacent to each other. The plates were allowed to dry in the dark: TSA for about one hour, and agarose overnight. Plates were visualized using a handheld UV lamp. Observations were recorded and pictures were taken using a cell phone camera.

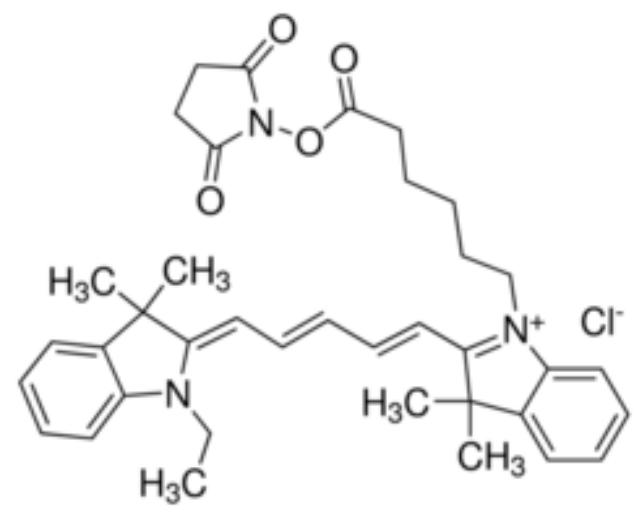

Figure 38 -Chemical structure for NIR-641 N-succinimidyl ester fluorescent dye; 1-Ethyl-1-[5-(Nsuccinimidyloxycarbonyl)pentyl]-3,3,3,3-tetramethyl-indodicarbocyanine chloride. 


\subsubsection{Data Analysis}

Basic analysis of averages, confidence intervals, and standard deviations was done with Microsoft Excel software.

\subsection{Results}

\subsubsection{Linear Fluorescent Range}

The linear range of fluorescence detection for fluorescein (FLX), Texas Red (TRX), and Cy5 labeled aptamers (CYX) was determined (Figure 39) LODs of 0.080, 1.32, and $0.806 \mathrm{nM}$ were determined for FLX, TRX, and CYX, respectively. LOQs of $0.265,4.41$, and 2.69 nM were determined for FLX, TRX, and CYX, respectively. The signal obtained was strongest for FLX, with TRX having the lowest signal. CYX had non-linear response below about $0.5 \mathrm{nM}$ and somewhat linear response below $5 \mathrm{nM}$. FLX and TRX did not lose linearity until the LOD.

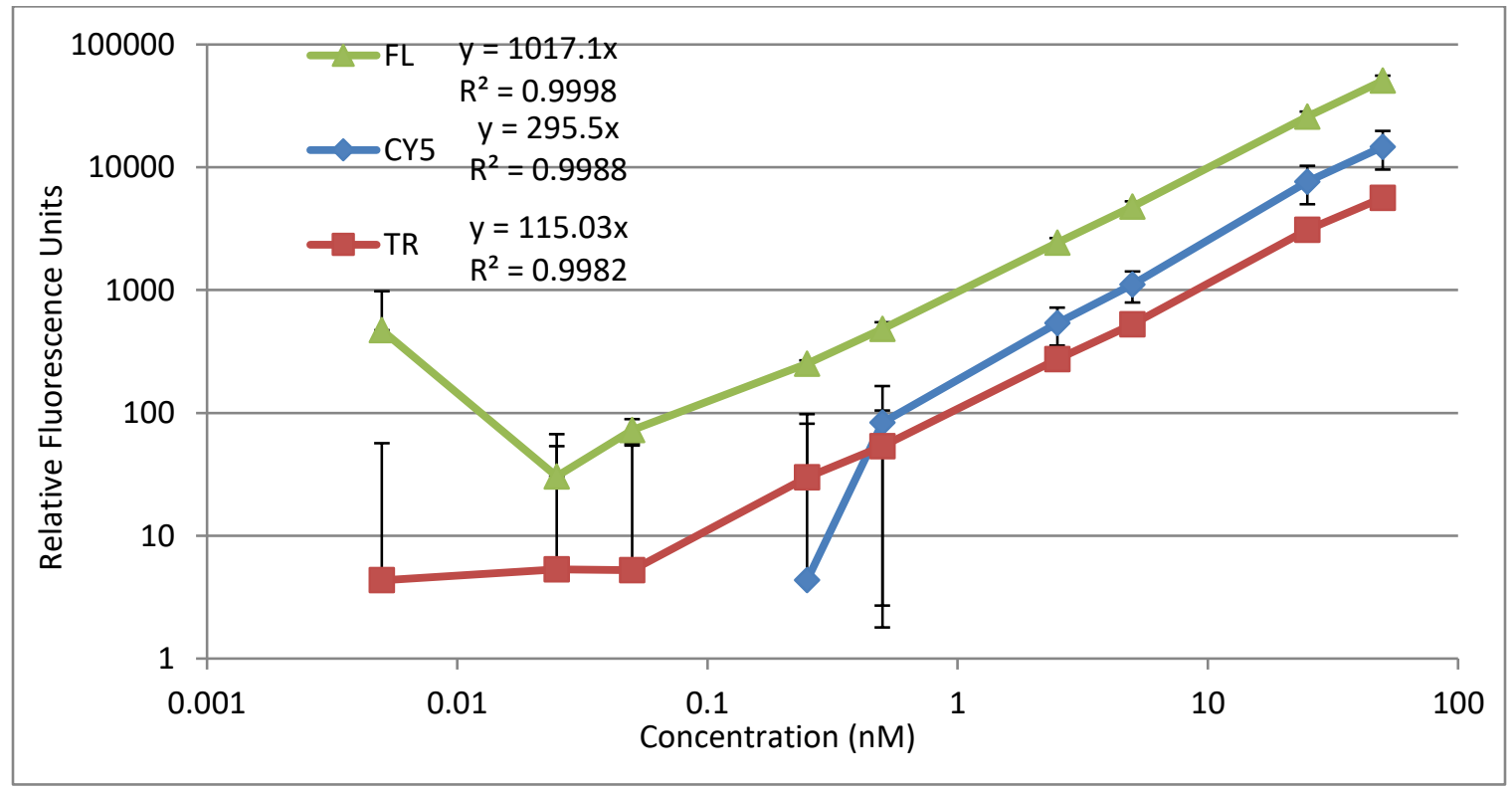

Figure 39 - Fluorescence of aptamers labeled with fluorescein (FL), Cy5, and Texas Red (TR) in TES buffer. Data points are the average of 9 samples over 3 trials. Error bars indicate SD. FL Ex $495 \pm 10 \mathrm{~nm}, E m 528 \pm 20$; TR Ex $596 \pm 15 \mathrm{~nm}, E \mathrm{~m} 680 \pm 30 \mathrm{~nm} ; C y 5 \mathrm{Ex} 620 \pm 40 \mathrm{~nm}, \mathrm{Em} 680 \pm 30 \mathrm{~nm}$ 


\subsubsection{Potential Fluorescence with Complete Complement}

TRQ and CYQ were tested to observe the maximum fluorescence possible in the presence of a complete complement DNA (CC). Fluorescent signal for TRQ in the presence of CC increased to about $80-90 \%$ of the signal of TRX (Figure 40). TRQ alone was found to be below the limit of detection. For CYQ in the presence of CC signal increased only about $40 \%$ of the value found for CYX (Figure 41). Signal for CYQ alone was at or below the limit of detection.

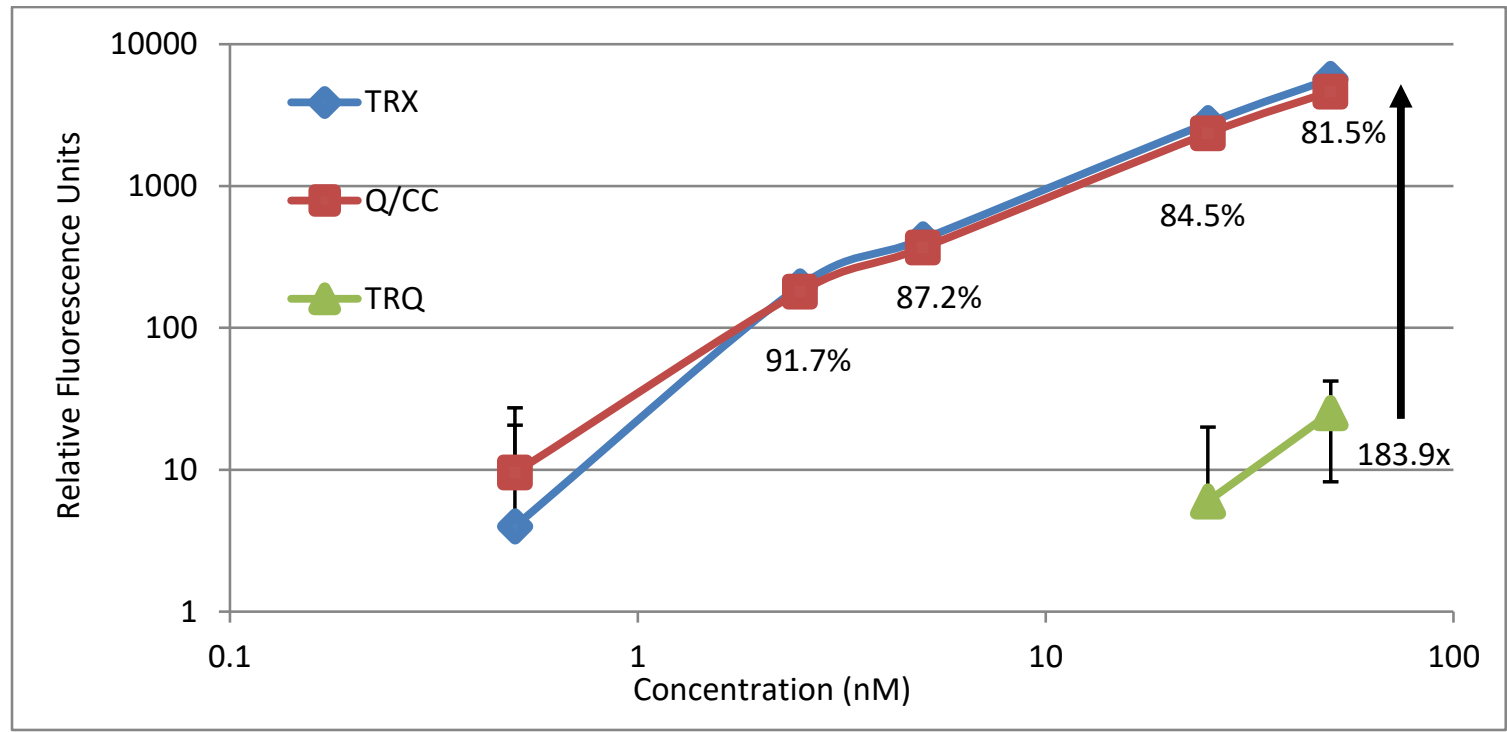

Figure 40 - Comparison of the fluorescence of Texas Red / Black Hole Quencher 2 labeled aptamer beacon (TRQ) in the presence of equimolar complete complement DNA (CC) compared to Texas Red labeled aptamer (TRX) and TRQ alone in TES buffer. Data points are the average of 9 wells over 3 replicates. Error bars represent SD. Ex $596 \pm 15 \mathrm{~nm}, \mathrm{Em} 680 \pm 30 \mathrm{~nm}$

\subsubsection{Interaction with VT}

The effect on fluorescence of interaction between TRQ aptamer beacon sequence ( $50 \mathrm{nM}$ ) and VT was investigated (Figure 42). At time 0 , TRX had fluorescence about 90 times higher than the quenched aptamer sequence and was somewhat stable before decreasing, which slowed over time (Panel A). The fluorescence of TRQ+CC 


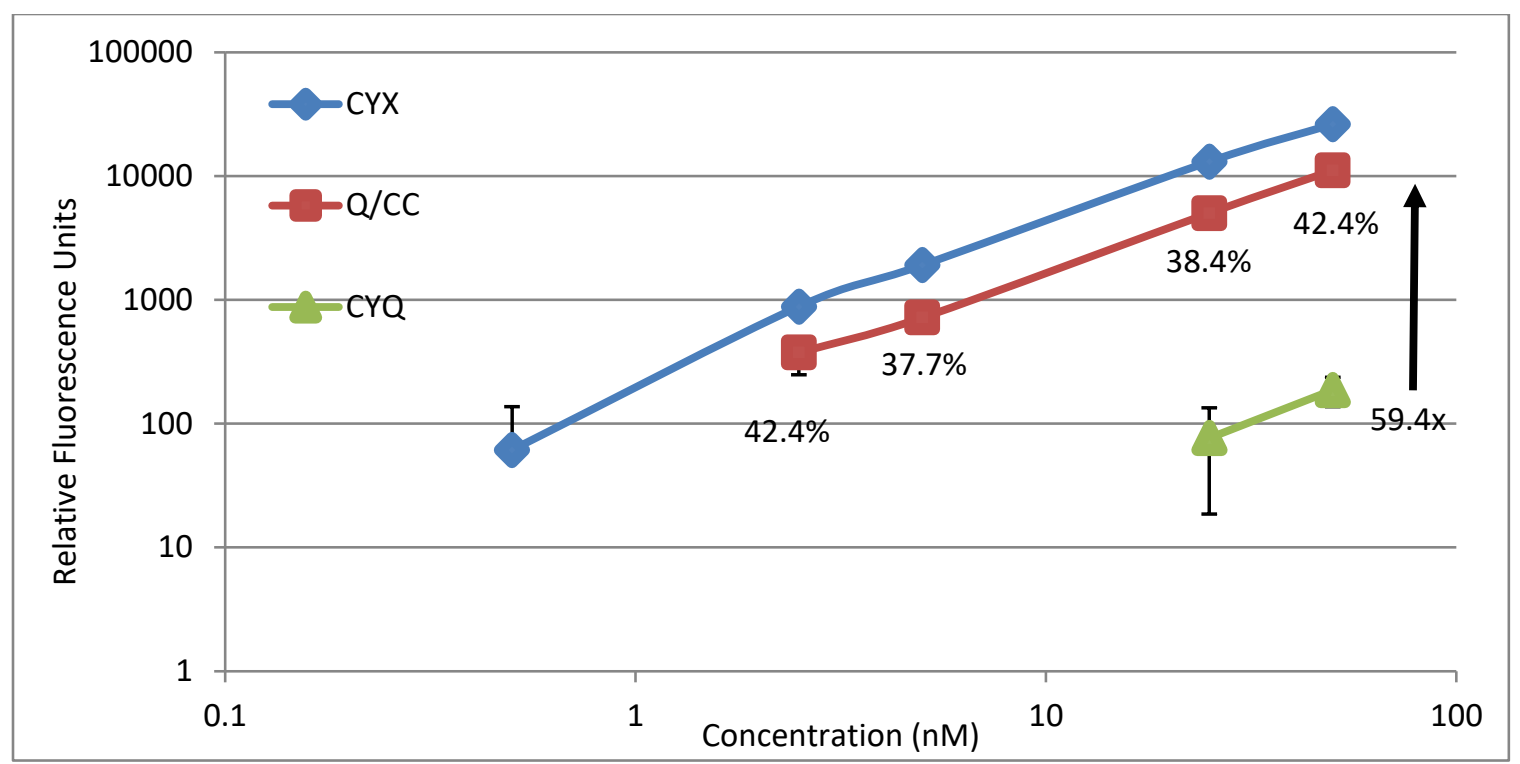

Figure 41 - Comparison of the fluorescence of Cyanine 5 / Black Hole Quencher 3 labeled aptamer beacon (CYQ) in the presence of equimolar complete complement DNA (CC) compared to Cyanine 5 labeled aptamer (CYX) and CYQ alone in TES buffer. Data points are the average of 9 wells over 3 replicates. Error bars represent SD. Ex $620 \pm 40 \mathrm{~nm}, \mathrm{Em} 680 \pm 30 \mathrm{~nm}$

increased rapidly to a peak at about 8 minutes with fluorescence about 20 times higher than TRQ without CC. After that point, the fluorescence remained somewhat stable and started to decrease. Fluorescence of TRQ and TRQ+VT2 decreased over time (Panel B). TRQ+VT1 started lower than TRQ alone, but slowly increased rather than decreasing.

The effect on fluorescence of interaction between CYQ $(50 \mathrm{nM})$ and VT was also investigated (Figure 43) and found to be very similar. The curve for CYX had about 65 times higher fluorescence than the quenched beacon at time 0 and decreased without a period of stable signal (Panel A). CYQ+CC fluorescence increased rapidly until it was about 15 times higher than CYQ alone then decreased. CYQ and CYQ+VT2 fluorescence decreased rapidly. CYQ+VT1 fluorescence started lower than CYQ alone but slowly increased rather than decreasing.

These experiments were attempted at $10 \mathrm{nM}$, however all data points involving 
quenched beacon were well below LOD and very little could be concluded (Figure A-15 and Figure $\mathrm{A}-16)$.

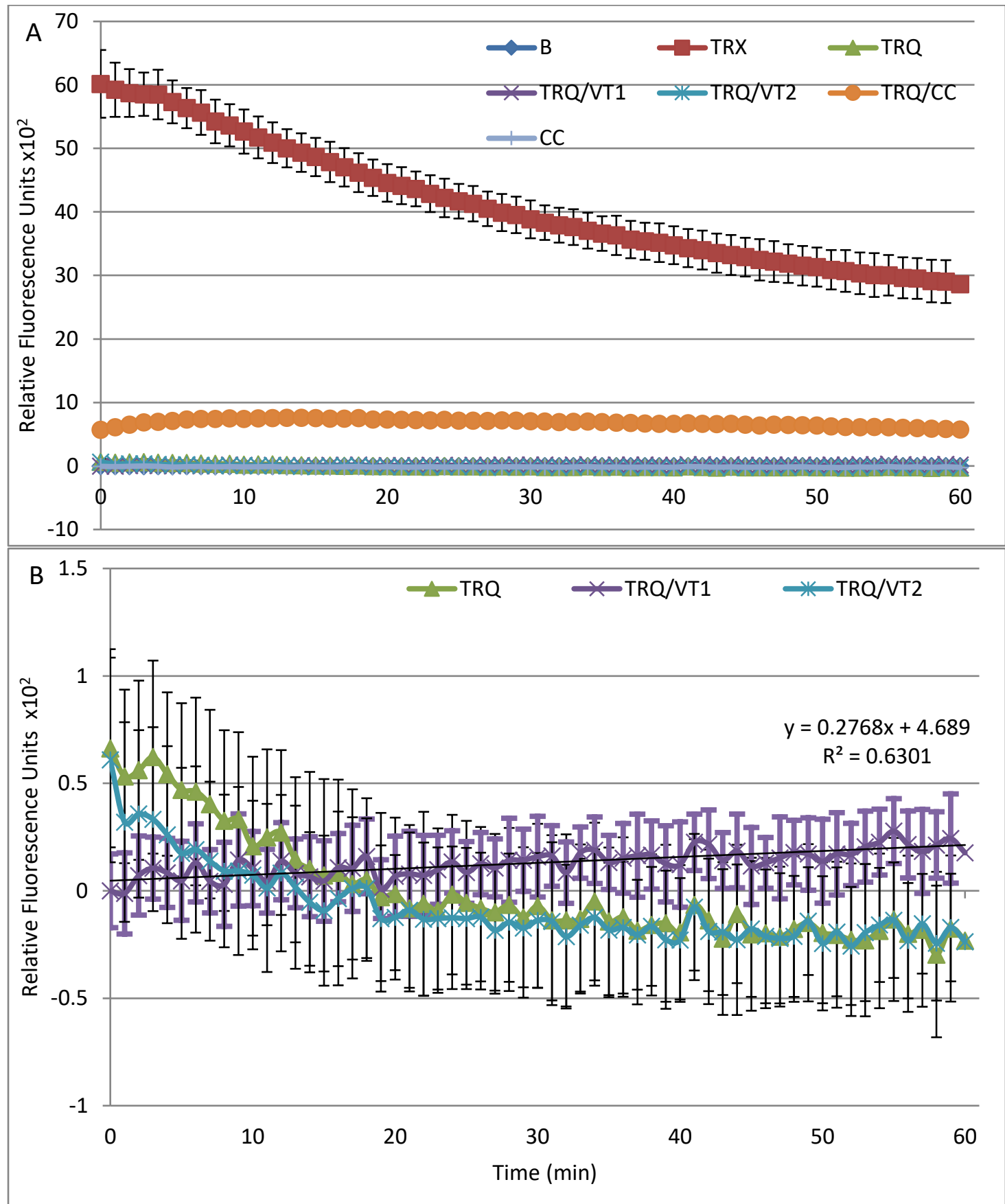

Figure 42 - Blanked change in fluorescence over time for Texas Red / Black Hole Quencher 2 labeled aptamer beacon (TRQ) (50 nM) in the presence of VT1a, VT2a, or complete complement (CC) each at $50 \mathrm{nM}$ in TES buffer. A) Full view including unquenched Texas Red labeled aptamer (TRX) sequence; B) view excluding complete complement DNA (CC) and TRX curves. Data points are the average of 9 readings over 3 replicates. Error bars show SD. Ex. $596 \pm 15$ nm, Em. $680 \pm$ $30 \mathrm{~nm}$. 


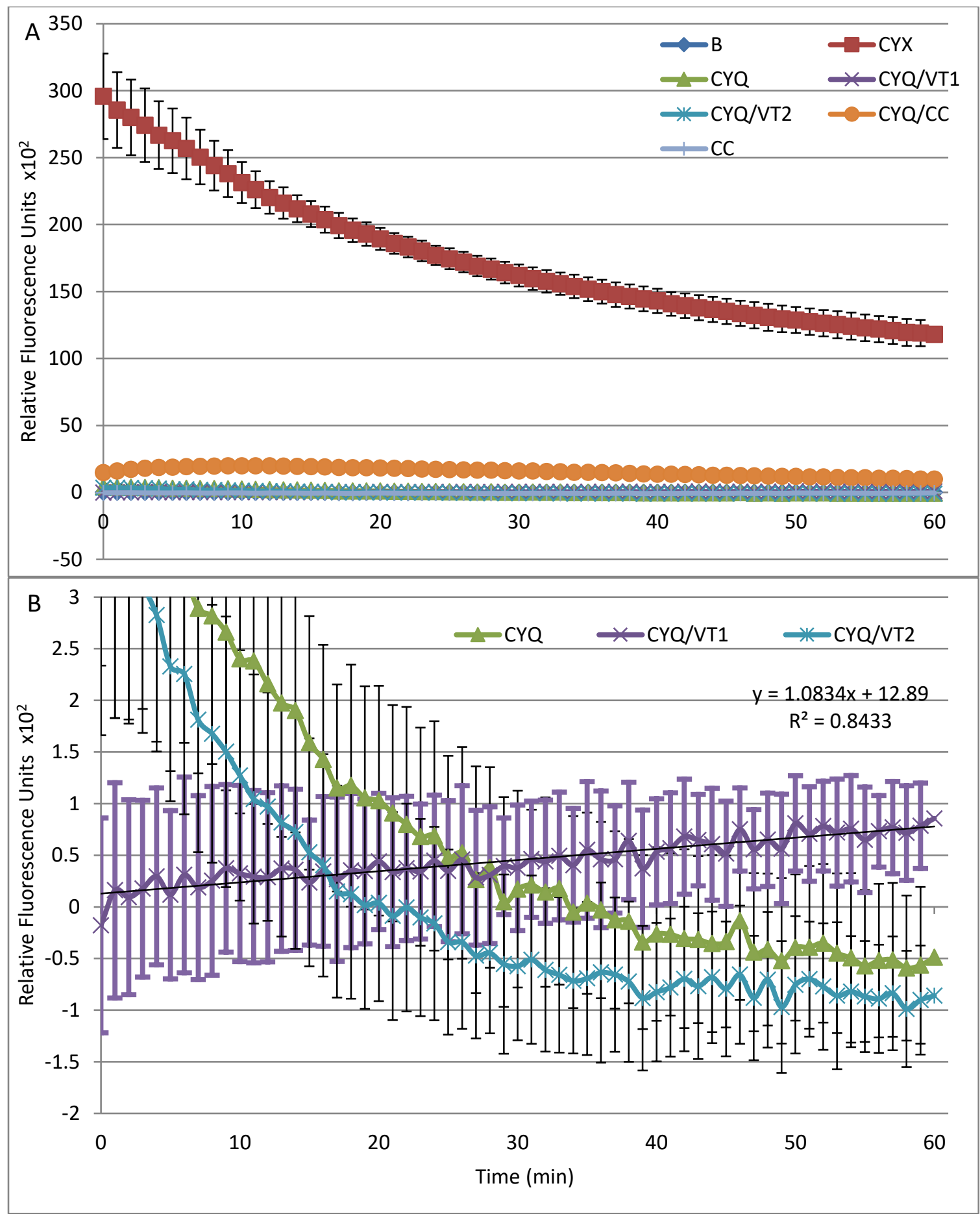

Figure 43 - Blanked change in fluorescence over time for Cyanine 5 / Black Hole Quencher 3 labeled aptamer beacon (CYQ) (50 nM) in the presence of VT1a, VT2a, or complete complement (CC) each at $50 \mathrm{nM}$ in TES buffer. A) Full view including unquenched Cyanine 5 labeled aptamer (CYX) sequence; $B$ ) view excluding complete complement DNA (CC) and CYX curves. Data points are the average of 9 readings over 3 replicates. Error bars show SD. Ex. $620 \pm 40 \mathrm{~nm}, \mathrm{Em} .680 \pm$ $30 \mathrm{~nm}$. 


\subsubsection{Comparison of Visible Limit of Detection}

Solutions of fluorescein (FI), Texas Red (TR), and NIR-641 (equivalent to Cy5)

were prepared in deionized water $(100-0.01 \mu \mathrm{M})$ to compare their visible fluorescence (Figure 44). Fl and TR were strongly visible at $100 \mu \mathrm{M}$, well visible at $10 \mu \mathrm{M}$, and slightly visible at $1 \mu \mathrm{M}$. Solutions less than $1 \mu \mathrm{M}$ did not have any visible fluorescence.

Additionally, NIR-641 did not show any visible fluorescence. Illumination with a green light was also attempted. In green light it appeared that Texas Red was somewhat fluorescent, however, NIR-641 still did not appear to fluoresce (Figure A-17).

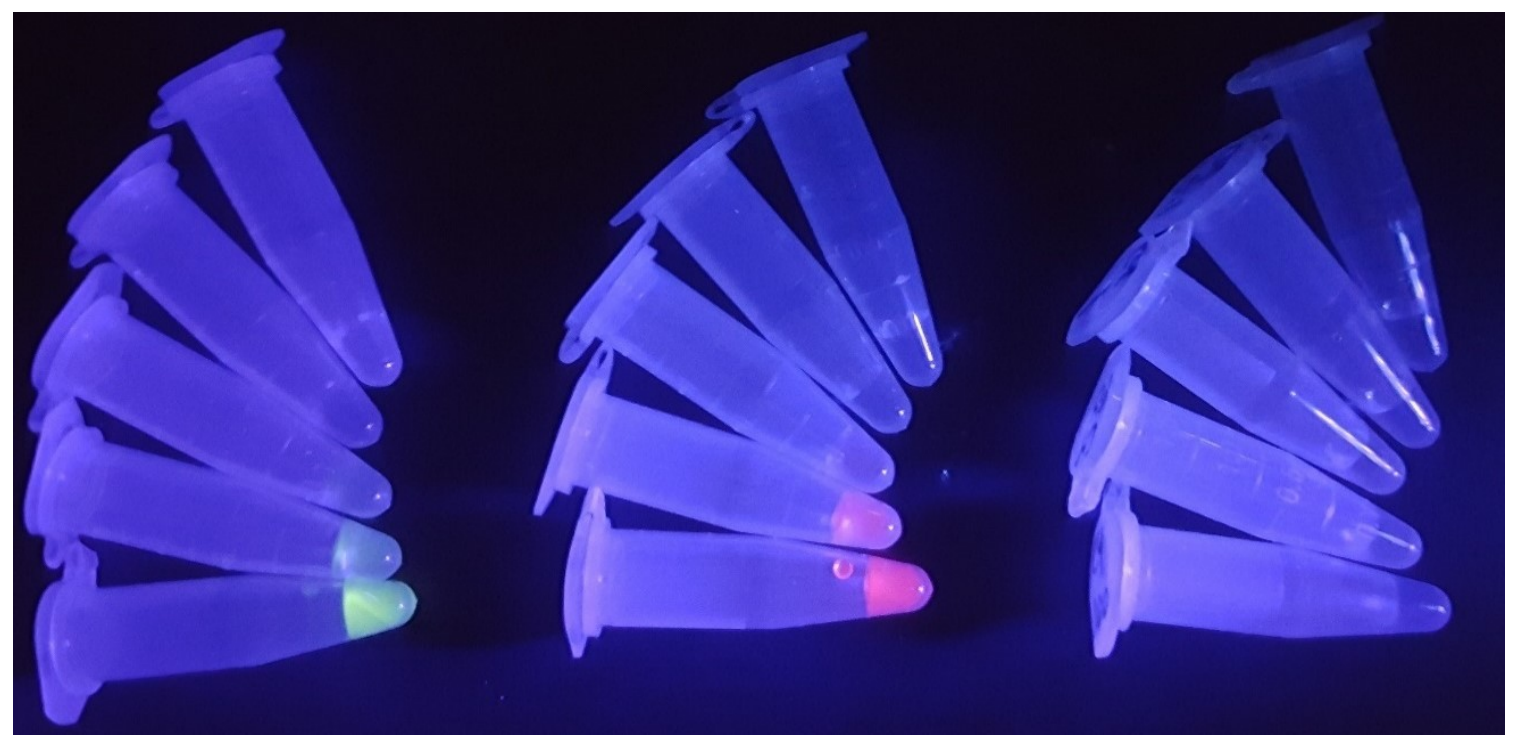

Figure 44 - Visible fluorescence of fluorescein, Texas Red, and Cy5 solutions from left to right $(100,10,1,0.1$, and $0.01 \mu \mathrm{M}$ from bottom to top) excited with a handheld UV lamp. Image taken with a cell phone camera.

Solutions of FI and TR were plated on TSA plates at concentrations of $40-1 \mu \mathrm{M}$ either on separate plates (Figure 45) or on the same plate (Figure 46). Fl fluorescence was at least somewhat visible down to about $20 \mu \mathrm{M}$. TR fluorescence was at least somewhat visible down to about $10 \mu \mathrm{M}$ and slightly at $5 \mu \mathrm{M}$. These TSA plates were a translucent beige in white light with slight green or pink spots visible (Figure A-18). 


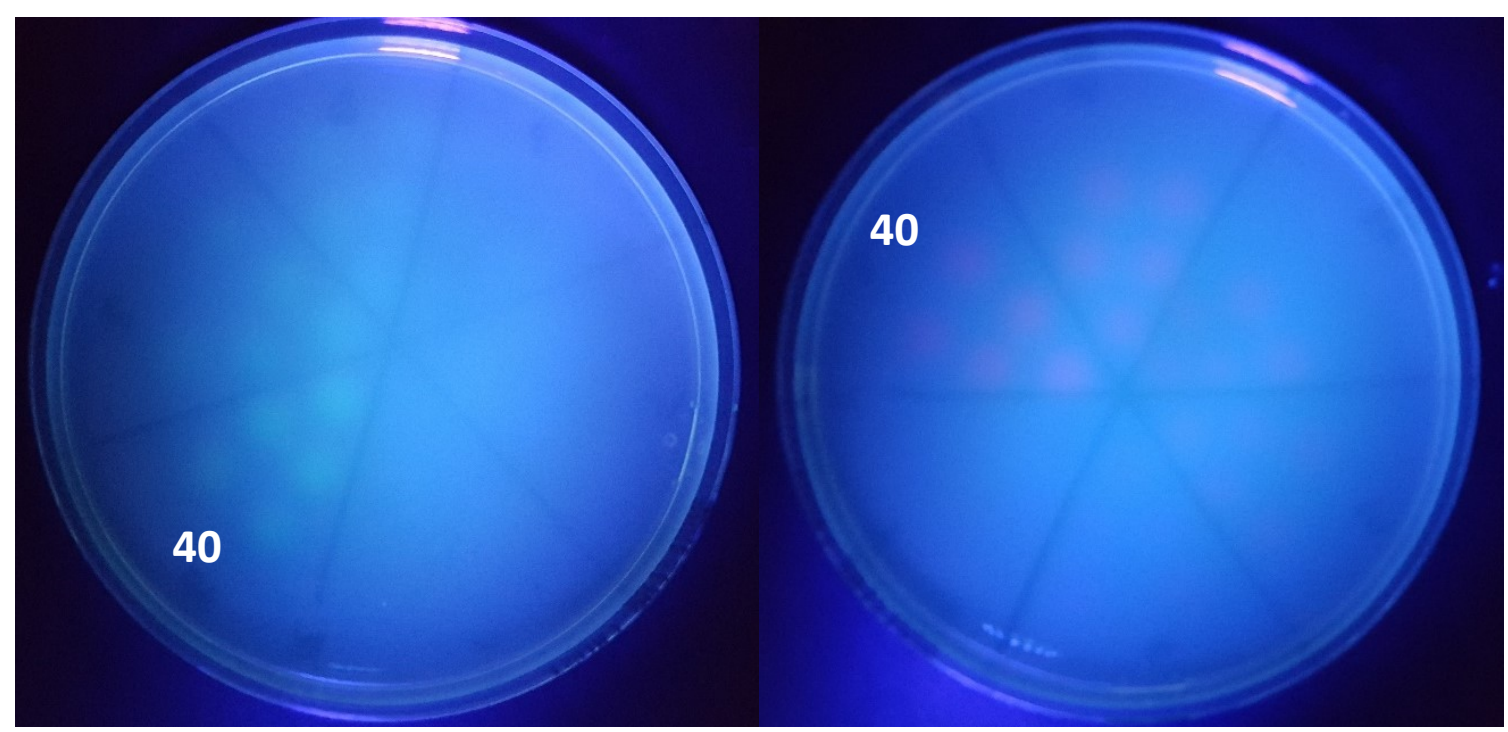

Figure 45 - Comparison of visible fluorescence of fluorescein (left) and Texas Red (right) spotted on untreated TSA plates and allowed to dry. Solutions were 40,30, 20, 10, 5, and $1 \mu \mathrm{M}$ going clockwise for each segment and spotted in quintuplicate. Image taken with a cell phone camera.

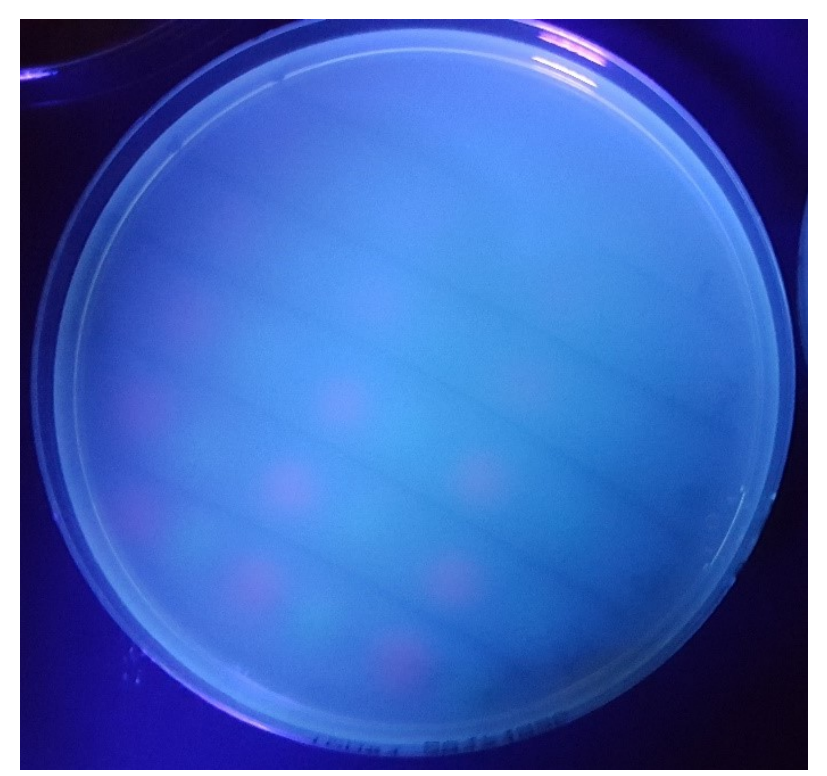

Figure 46 - Comparison of visible fluorescence of fluorescein and Texas Red spotted on an untreated TSA plate. Solutions were 40, 30, 20, 10, 5, and $1 \mu \mathrm{M}$ from bottom to top for each segment and spotted in triplicate. Image taken with a cell phone camera.

The same experiment was repeated on $1.5 \%$ agarose plates and left overnight to dry (Figure 47). Spotted fluorophore did not result in distinct circles of fluorescence, but rather in smears. Fluorescein was at least somewhat visible at 20 and $10 \mu \mathrm{M}$. Texas Red 
however was not distinctly visible. There appeared to be what might be fluorescence at 40,20 , and $10 \mu \mathrm{M}$, but this was difficult to say confidently since the auto-fluorescence of the $1.5 \%$ agarose was a similar pink-purple colour. The plate that had the solutions spotted side by side had no visible fluorescence (Figure A-19).

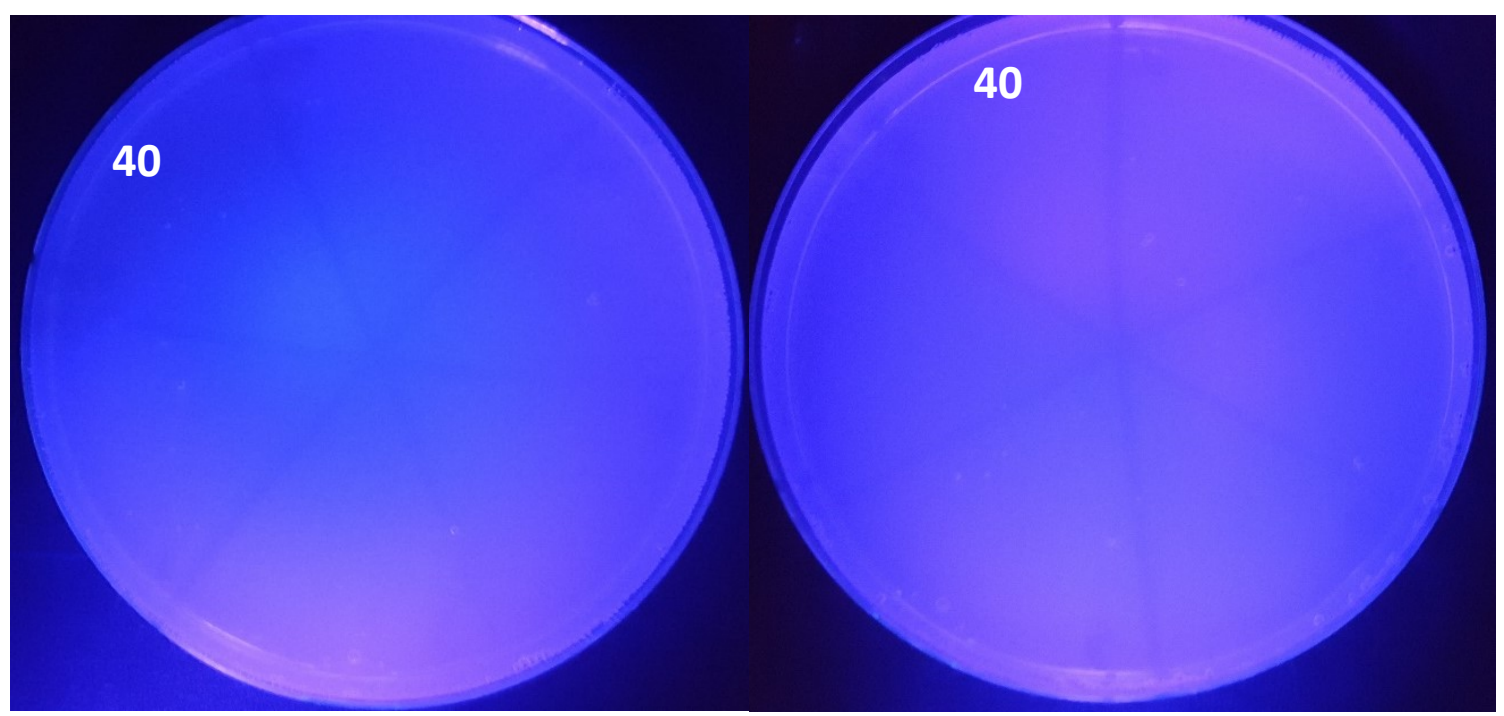

Figure 47-Comparison of visible fluorescence of fluorescein (left) and Texas Red (right) spotted on untreated 1.5\% agarose plates. Solutions were 40, 30, 20, 10, 5, and $1 \mu \mathrm{M}$ going clockwise for each segment. Samples were spotted in quintuplicate and plates were refrigerated overnight to allow spots to dry. Images taken with a cell phone camera.

\subsection{Discussion}

\subsubsection{Linear Fluorescent Range}

The linear range of fluorescence was determined for FLX, TRX, and CYX (Figure 39). Concentrations above $50 \mathrm{nM}$ were not tested due limited reagents. FLX was found to be the most sensitive and produced the strongest detected signal. One likely reason for this was the excitation and emission filter sets used in this experiment that were optimal for fluorescein excitation and emission (Figure 48). For fluorescein both excitation (495/10 nm) and emission (528/20 nm) filter wavelengths were near its peak 
absorption and emission with a predicted $33 \%$ of total emission detected. For Texas Red the excitation wavelengths $(596 / 15 \mathrm{~nm})$ were at the peak absorption, however, due to complications with emission filters the one used was optimal for Cy5 emission (680/30 $\mathrm{nm}$ ) with only about $10 \%$ of total emission predicted to be detected. This would result in

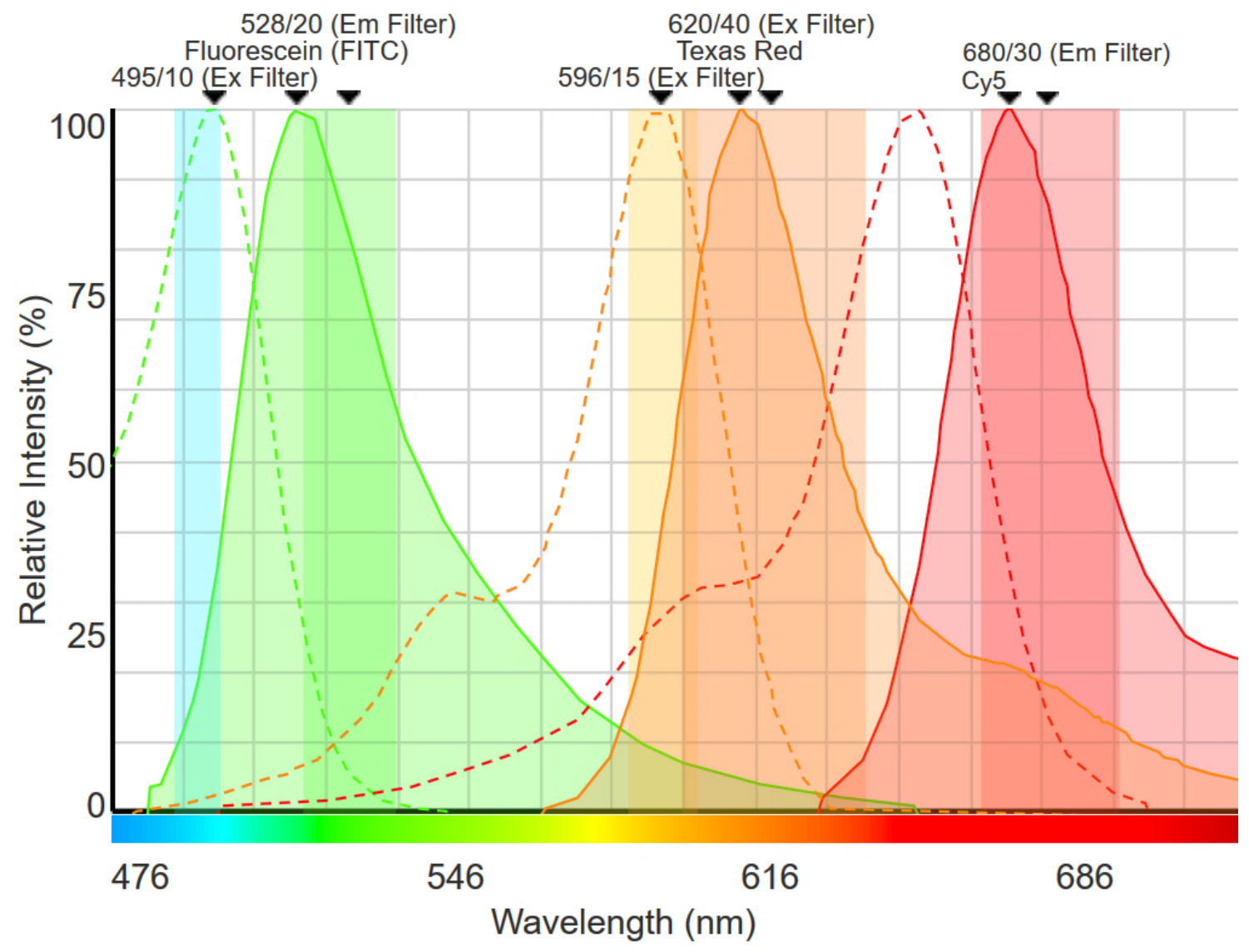

Figure 48 - Fluorescence spectra of fluorescein, Texas Red, and Cy5 as well as excitation and emission filters ranges. Absorption spectra are indicated by dashed lines and emission spectra by solid lines. Image made using ThermoFisher's Fluorescence SpectraViewer Web Application.

much lower sensitivity for Texas Red. Cy5 had the opposite problem, the excitation wavelengths (620/40 nm) were less than optimal, not reaching the peak absorption. This would result in lower induced fluorescence. The emission filter was predicted to detect about $47 \%$ of total emission. Although this causes difficulty in comparing absolute fluorescence of the fluorophores, what is more important is the signal contrast between 
the quenched aptamer beacons in buffer and the beacons with VT. Those values should not change, regardless of filter sets. Additionally, the absolute fluorescence would be compared when observed on solid media.

Another factor expected to lead to low sensitivity in this experiment in general was the 50/50 mirror being used instead of dichroic mirrors. Dichroic mirrors have special coatings that allow only wavelengths below the cut-off value into the sample and those above to be detected. This minimizes background signal from any other light or contaminants. A 50/50 mirror, however, allows 50\% of excitation light into the sample, and $50 \%$ of emission light to be detected regardless of wavelength. This makes comparison of different fluorophores easier since only a single mirror would be needed. However, only about $25 \%$ of the fluorescence is detected (Figure 49 ). The reduced specificity of wavelengths detected causes higher background, as well as lower sensitivity due to loss of excitation and emission light. However, the same LOD for FLX was calculated for both a 50/50 mirror and a $510 \mathrm{~nm}$ dichroic mirror (0.080 nM). As such, the 50/50 mirror appears to only minorly affect the sensitivity of the fluorescence.

Finally, although both fluorescein and Texas Red appeared to have linear fluorescence until their LOD, Cy5's fluorescence was much less linear, particularly below $10 \mathrm{nM}$. The non-linear fluorescence became less linear as concentration decreased. Concentrations above $10 \mathrm{nM}$ should be sufficiently accurate for Cy5. However, the experimental concentration needs to be higher since quenchers will be used. 


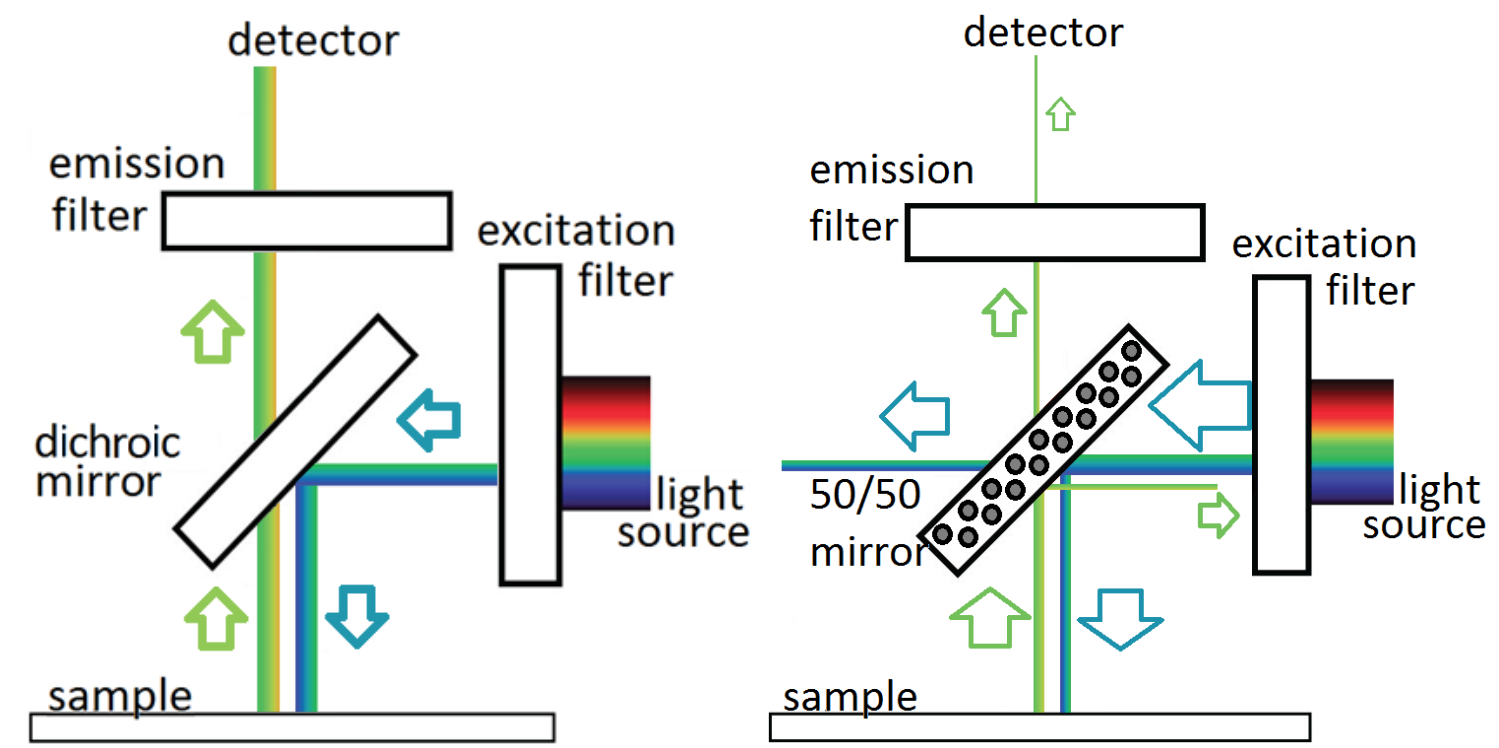

Figure 49 - Comparison of dichroic and 50/50 mirrors used for fluorescence detection. Left: schematic of the use of a dichroic mirror. Right: schematic of the use of a 50/50 mirror.

\subsubsection{Potential Fluorescence with Complete Complement}

CYQ and TRQ were tested with CC to see if they would produce their maximal

fluorescence. Previously FLQ had shown complete fluorescence above $2.5 \mathrm{nM}$. In comparison TRQ (Figure 40) showed 81.5-91.7\% fluorescence and CYQ (Figure 41) only resulted in a $37.7-42.4 \%$ signal from $2.5-50 \mathrm{nM}$. It was unclear what would cause such low signal for $\mathrm{CYQ}$ since the sequences and complement were the same. To a small extent quenching by neighbouring bases would make Cy5's fluorescence lower while Texas Red is less affected by this parameter, however, it does not account for the $\sim 50 \%$ difference in signal seen. Another potential reason for this was that BHQ3's peak absorption is much closer to the peak emission for Cy5 than BHQ2 and Texas Red. When comparing increase in signal at $50 \mathrm{nM}$ FLQ previously increased about 7.16x, CYQ increased 59.4x, and TRQ increased about 183.9x. Both pairs were much better than the previous contrast with fluorescein / dabcyl. However, Texas Red seems much more 
consistent and has a significantly higher contrast between background and induced signal. For this reason, it appears that Texas Red is a much better choice for use in the aptamer beacon.

Additionally, for both TRQ and CYQ the signal produced by the quenched beacon alone was at or below the detection limit. Only samples at 25 and $50 \mathrm{nM}$ were strong enough to plot on the graphs. This suggests that the Black Hole Quenchers were much more effectively quenching the fluorophores than dabcyl had previously. This was expected from the spectral overlaps being much more optimal (Table 6).

\subsubsection{Interaction with VT}

Interaction at $50 \mathrm{nM}$ of both TRQ and CYQ with VT (1:1 ratio) was investigated (Figure 42 and Figure 43). Overall, the shape of the curves was very similar to that previously seen with fluorescein: unquenched aptamer signal decreased rapidly, quenched beacon and VT2 decreased slowly, VT1 started lower and stayed stable until it eventually had a stronger signal than the quenched beacon alone. This strongly suggests that the sequence itself was the main cause of poor signal in the presence of toxin.

Of note, the background fluorescence was much less than for fluorescein (Figure 17). For fluorescein the background was about $8 \%$ of the unquenched aptamer, while only about $1.1 \%$ and $1.5 \%$ for TR and Cy5, respectively. This again suggests that the Black Hole Quenchers were more effectively quenching than dabcyl had been. Potentially for the fluorescein labeled beacon a quencher such as BHQ1 (peak absorption around $534 \mathrm{~nm}$ ) could more effectively quench its fluorescence to obtain similar reduced background. Since the signal in presence of VT was so low this enhanced 
quenching caused a problem for detection. The samples at $50 \mathrm{nM}$ were near the limit of detection, or slightly below. The experiment was attempted at $10 \mathrm{nM}$ levels for the reagents to compare with fluorescein, but the signal was so low that no conclusive observations could be made (Figure A-15 and Figure A-16).

The signal observed for CC samples was about $15.4 \%$ at 14 minutes for TR and $8.6 \%$ at 10 minutes for Cy5, compared to the unquenched aptamers. The difference between TR and Cy5 was similar to that seen for the maximum fluorescence in Figure 41 and Figure 40. Likely the same factors were causing this lower enhanced signal for CYQ.

The amount of signal loss for the unquenched aptamers was less than previously observed for fluorescein (Table 7). Although less, photobleaching was still a major factor if measurements were to be taken over a period of time. Texas Red, being the most stable of the three fluorophores (Table 6) was seen to have the least loss of fluorescence signal and additionally both TRX and TRQ+CC samples have regions of stability not seen with the equivalent Cy5 samples.

Table 7 - Comparison of unquenched signal loss over 1 hour in buffer

\begin{tabular}{|l|c|c|}
\hline Fluorophore & Signal Decrease in 1 hour & Figure \\
\hline Fluorescein (FLX) & $63 \%$ & Figure 26 \\
\hline Texas Red (TRX) & $52 \%$ & Figure 42 \\
\hline Cyanine 5 (CYX) & $59 \%$ & Figure 43 \\
\hline
\end{tabular}

\subsubsection{Comparison of Visible Limit of Detection}

Solutions of free fluorescein (FI), Texas Red (TR), and NIR-641 (Cy5 equivalent) were prepared to find a minimum visible fluorescence. Illumination by a handheld UV lamp caused FI and TR to be visible at concentrations down to $1 \mu \mathrm{M}$, but NIR-641 
fluorescence was not visible (Figure 44). In solution, FI and TR were of a similar intensity of fluorescence. One potential reason for NIR-641's lack of fluorescence was that Cy5 required longer wavelengths for excitation. Illumination of NIR-641 with a green light did not improve visible fluorescence (Figure A-17). CYX was also illuminated with UV and green light and no fluorescence was observed (not shown). As such it was likely that longer excitation wavelengths were required for excitation.

Fluorescein and Texas Red were plated on TSA agar plates. Fluorescein was somewhat discernable at $20 \mu \mathrm{M}$ while Texas Red was quite clearly discernable at $10 \mu \mathrm{M}$ and maybe slightly visible at $5 \mu \mathrm{M}$ (Figure 45 and Figure 46). As expected, a fluorescence that was more red-shifted had a higher contrast on the blue-green auto-fluorescing TSA plates. For use on TSA, Texas Red would be a better choice in beacons.

The same experiment was repeated for $1.5 \%$ agarose plates. Plates were left overnight to dry resulting in smeared fluorescence rather than spots (Figure 47). Smearing of the fluorescence would occur less for fluorophore labeled aptamers since they have a much larger mass, limiting free movement. Unlike TSA, 1.5\% agarose plates auto-fluoresced a pink-purple colour. In this case Texas Red was much harder to discern, making fluorescein a better choice for use in beacons applied to $1.5 \%$ agarose. This suggests that, aside from fluorophore stability and signal intensity, the media being used would strongly affect the effectiveness of a fluorophore used for detection of VTEC. Other media may have other colours of auto-fluorescence to consider. 


\subsection{Conclusions}

Experimentation of aptamer beacon labeled with Texas Red / Black Hole Quencher 2 and Cyanine 5 / Black Hole Quencher 3 was investigated as a means of improving signal in the presence of VT. Both had lowered background fluorescence and increased signal contrast compared to the fluorescein / dabcyl beacon. This indicated that Black Hole Quenchers would be preferable for future beacon design. Texas Red, however, was much more stable and reliable than Cy5 and would be a preferable fluorophore for general use. Experimentation in complex media was not pursued due to limited reagents, but should be tested to confirm their suitability for future applications.

Interaction between the aptamer beacons and VT was found to be the same as previously found with fluorescein / dabcyl beacon in Chapters 2.0 and 3.0, showing minimal signal increase. Use of the alternate fluorophore / quencher pairs also made it clear that changing the labels was not going to affect the interaction between the beacon and VT.

For use on solid media in isolation of VTEC the fluorophores were tested on TSA and $1.5 \%$ agarose. With a UV lamp fluorescein and Texas Red fluorescence intensity were equally strong, however, each was much more visible on a different medium. Selection of a fluorophore likely depends on the desired growth media being used.

Overall, Texas Red labeled beacons appear to be the most optimal choice for use in buffer or on TSA plates. If a VT aptamer beacon was designed in the future this would be the best choice for use screening filtrates or potentially for application by spotting aptamer beacon solution onto colonies for isolation. 


\subsection{PolyA DNA Substrate for Enzymatic Activity Differentiation}

\subsection{Introduction}

\subsubsection{Use of VT Enzyme Activity as a Detection Method for VT2}

Verotoxin is an $A_{5}$ toxin composed of an enzymatically active $A$ subunit which has $\mathrm{N}$-glycosidase activity and cleaves adenine from adenosine residues, and five $\mathrm{B}$ subunits which bind Gb3 residues to assist in toxin internalization. Generally, the toxin must be cleaved to allow access to the active site of the A1 subunit. Aside from the primary in vivo activity on rRNA substrates, activated VT1 has been shown to be able to cleave adenine residues from a variety of RNA and DNA substrates (Barbieri et al., 1998). This is done by formation of abasic sites leading to DNA breakdown and potentially cleavage due to a weakened DNA sugar-phosphate backbone following extensive depurination (Brigotti et al 2001).

Ricin and some other type II RIPs were observed to have DNA depurination activity prior to separation of the enzymatic subunit (Barbieri et al., 1997). Although VT1 required cleavage (Barbieri et al., 1998) the activity of VT2 on DNA has not yet been demonstrated. The different structure of VT2 A subunits may allow for activity without cleavage. Through x-ray crystallography it was found that the VT1 A1 active site is blocked by part of cleavable loop chain that links the A1 and A2 subunits, however, the VT2 A1 active site is more accessible (Fraser et al., 1994; Fraser et al., 2004). As such VT2 A subunit may be enzymatically active without cleavage. VT2 was seen to cleave adenosine without reduction (Fraser et al., 2006). Additionally, VT has been shown to be released from VTEC not as holotoxins, but primarily as individual subunits (Pellino et al., 
2016). This suggested that VT2 A subunits could be enzymatically active on smaller DNA substrates in solution.

Cleavage of DNA backbone at abasic sites can be catalysed by the addition of amino compounds (McHugh and Knowland, 1995). This is accomplished through a beta cleavage reaction at the $5^{\prime}$ phosphate (Figure 50), in which an uncharged amine group is reduced. The $\mathrm{pK}_{\mathrm{a}}$ of the amino group must be low enough to ensure it is uncharged under the reaction conditions. Compounds with more than one amino group are more effective catalysts at lower concentrations and near neutral $\mathrm{pH}$ (McHugh and Knowland, 1995). This is facilitated by the positive charge on one amino group promoting binding to DNA, while the uncharged amino group can then catalyze the cleavage.

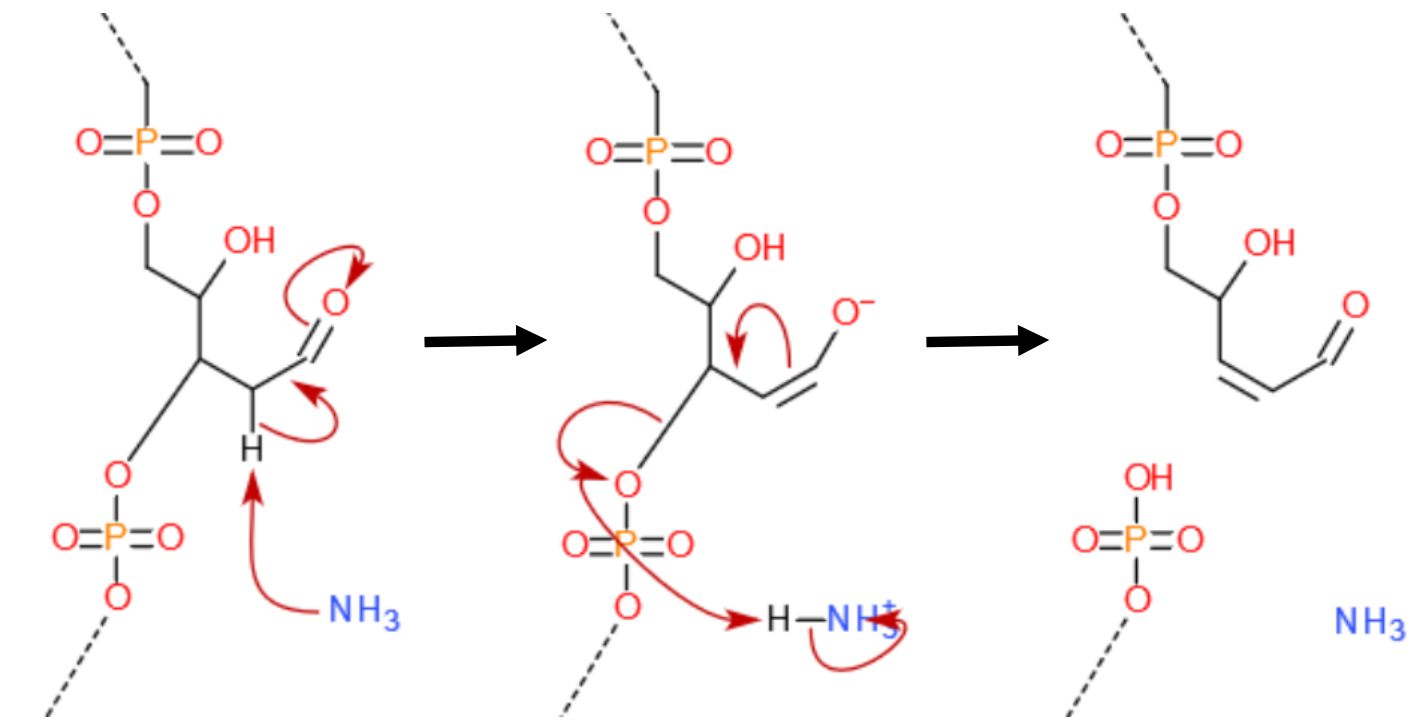

Figure 50 - Mechanism for amine-catalyzed B-elimination causing DNA backbone cleavage at abasic sites.

A simple nucleotide that could act as a substrate for VT2 could potentially be used as a method of detection for VTEC. VT2 A subunits released from VTEC could potentially react with such a substrate without additional processing. Labeling with fluorophore and quencher would make the substrate a turn-on sensor in the case of 
DNA cleavage. Addition of amino compounds could further enhance cleavage of the backbone to increase sensitivity.

\subsection{Chapter Objectives}

This chapter investigated the potential of a DNA-based fluorophore / quencher labeled substrate as a means of detection through the catalytic activity of VT. The use of amine catalyzed cleavage was used to promote any minor activity.

\subsection{Materials and Methods}

\subsubsection{Buffer}

Based on previous published enzyme activity experiments performed to observe VT enzyme activity (Basu et al., 2016; Brigotti et al., 2001; Brigotti et al., 1998; Barbieri et al., 1998; and Endo et al., 1990) and the need for cofactors for a DNase positive control the buffer was chosen to be the following:

Enzyme Activity Buffer (EAB): $20 \mathrm{mM}$ tris- $\mathrm{HCl}, 0.5 \mathrm{mM} \mathrm{MgCl}$, $\mathrm{pH} 7.4$

\subsubsection{DNA Substrate - polyA}

To serve as a substrate for detection a short, A-rich, DNA sequence was developed dual labeled with fluorescein and Black Hole Quencher 1. The substrate, referred to as "polyA", was of the sequence 5'- 6-FAM GTAG AAA AAA AAA AAA CTACBHQ1 - 3'. PolyA substrate was ordered from Alpha DNA (Montreal, QC). 


\subsubsection{Activity Assay}

Equal volumes of enzyme activity buffer, DNase (Promega L), commercial VT1a, and commercial VT2a were mixed separately with the polyA substrate to result in solutions with 1 unit of DNase or $10 \mathrm{nM}$ of toxin, and $3.75 \mu \mathrm{M}$ polyA. A control with each reagent was also prepared. Each solution was pipetted in triplicate into a 96-well plate. The solutions were incubated at $30^{\circ} \mathrm{C}$ for 40 minutes with fluorescent readings taken each minute. Fluorescence readings were taken by a Synergy 2 Multimode Plate Reader (Biotek) equipped with a top 510 dichroic mirror, excitation filter: 495/10 nm, and emission filter: $528 / 20 \mathrm{~nm}$, at a reading sensitivity of 50 . After the 40 -minute incubation $2.17 \mu \mathrm{L}$ of $\mathrm{N}, \mathrm{N}^{\prime}$-dimethyl-ethylene-diamine (Sigma-Aldrich) was added to each well resulting in a concentration of $100 \mathrm{mM}$. The plate was incubated again at 30 ${ }^{\circ} \mathrm{C}$ for 40 minutes while readings were taken each minute. This was repeated twice more, although once with only duplicate wells due to limited polyA reagent.

\subsubsection{Data Analysis}

Basic analysis of averages, confidence intervals, and standard deviations was done with Microsoft Excel software.

\section{$5.4 \quad$ Results}

\subsubsection{DNA Substrate Design}

A nucleotide substrate for VT was designed taking into consideration VT activity, substrate stability, and available modified amidites. The substrate chosen for the experiments was of the sequence: 5' - 6-FAM GTAG AAA AAA AAA AAA CTAC-BHQ1 - 3' 
(Figure 51). A stable hairpin formation was predicted for the sequence with a predicted melting temperature of $34.9^{\circ} \mathrm{C}$.

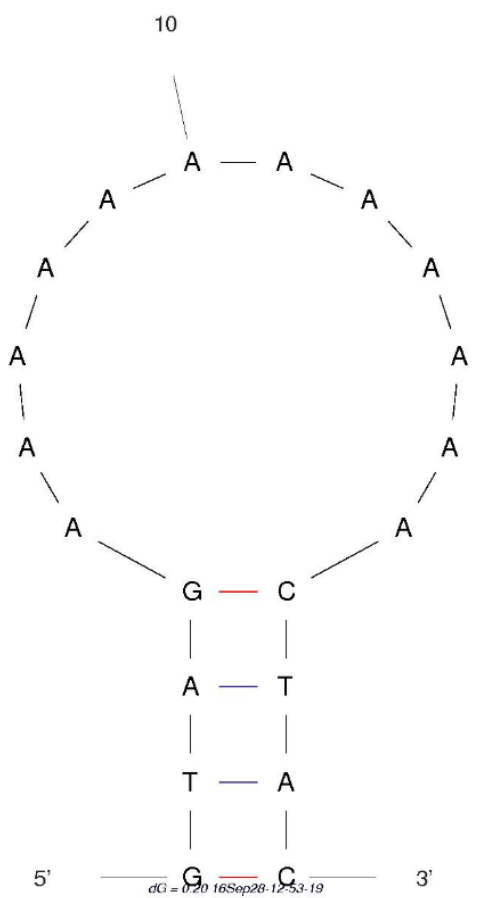

Figure 51 - Predicted secondary structure of polyA substrate using Mfold software (Zuker, 2003).

\subsubsection{Enzyme Activity Assay}

Enzyme activity on polyA substrate was tested with the addition of DNase, commercial VT1a, or commercial VT2a. DNase activity was confirmed by activity on genomic DNA (not shown). Fluorescence readings were taken for 40 minutes while incubated at $30^{\circ} \mathrm{C}$ before and after addition of N,N'-dimethyl-ethylene-diamine. Initial and final fluorescent values were blanked by polyA background (Figure 52).

Fluorescence for VT and DNase samples with polyA at time 0 were higher than that of polyA alone. During the first incubation, only DNase fluorescence values were increased. Initial values for all samples with polyA were higher after addition of amine. During the second incubation, DNase no longer increased fluorescence. Only VT2 solutions were 
seen to increase during the second incubation. Initial fluorescence was variable between replicates (Figure A-20 to Figure A-25). Individual replicates always had similar curve shapes with VT2 always being the only one increasing more than background. Crude VT2a was also tested with results less substantial than commercial VT2. The activity of crude VT2a was not tested prior to testing with the substrate.

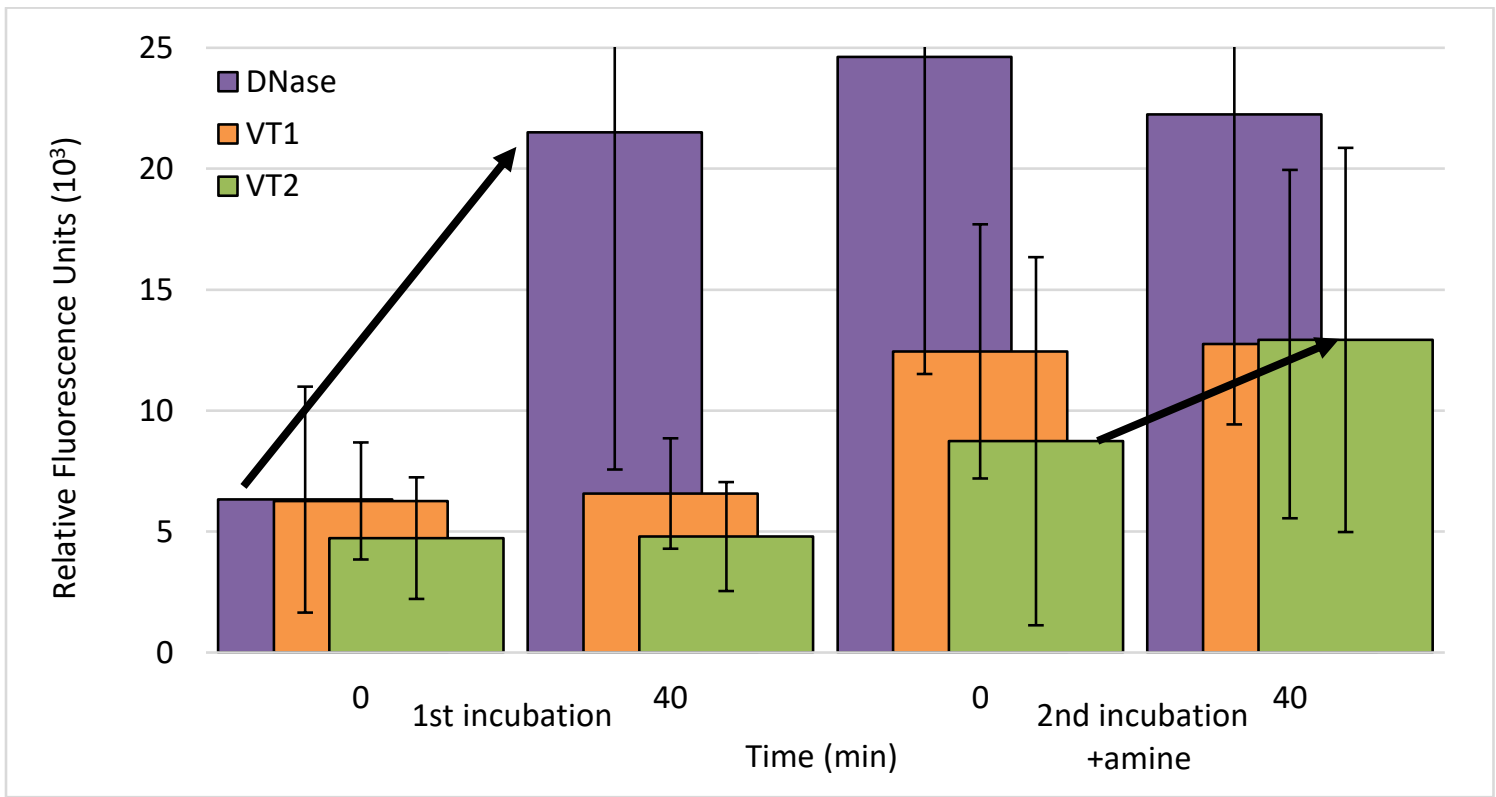

Figure 52 - Mean fluorescence values for the enzyme activity assay at time 0 and 40 for both 40minute incubations at $30^{\circ} \mathrm{C}$ with polyA background subtracted. Solutions in EAB contained DNase (0.5 units per $100 \mu \mathrm{L})$, VT1 or VT2 (10 nM), polyA (3.75 $\mu \mathrm{M})$ ), and during the second incubation $N, N^{\prime}$-dimethyl-ethylene-diamine $(100 \mathrm{mM})$. Data points the average of 8 samples over 3 replicates. Error bars show original SD. Ex. $495 \pm 10 \mathrm{~nm}, \mathrm{Em} .528 \pm 20 \mathrm{~nm}$.

\subsection{Discussion}

\subsubsection{Substrate Design}

Since VT's enzymatic activity cleaves adenine residues, ideally a fluorophore or quencher could be attached to an adenine residue in a short oligonucleotide replicating the conformation of the SRL region on 28S rRNA (Endo et al., 1988a). Upon cleavage the labeled adenine would be released from the substrate with the adenine inducing 
fluorescence. However, no suitable quencher or fluorophore labeled adenine residues were available. An amino-modified adenine as well as some intrinsically fluorescent adenosines were available which could be further investigated or used in future experiments. In this case a hairpin probe was designed which would separate as the backbone is cleaved. A substrate primarily made of adenine would allow for the most abasic sites to be formed and potentially cleave as a result of major adenine removal. With VT having activity on both DNA and RNA substrates (Barbieri et al., 1998), DNA was preferred for the substrate as a more stable option.

Substrates with only A and T residues were not predicted to form a single stable conformation so $\mathrm{C} / \mathrm{G}$ bases were added to the hairpin region. The substrate chosen for the experiments was of the sequence: 5' - 6-FAM GTAG AAA AAA AAA AAA CTAC-BHQ1 $3^{\prime}$. This sequence provided a sufficiently stable hairpin formation with a predicted melting temperature of $34.9^{\circ} \mathrm{C}$ (Figure 51). This is high enough above room temperature to minimize background fluorescence, while also short enough to likely separate when cleaved (Tyagi and Kramer, 1996).

Fluorescein and Black Hole Quencher 1 (BHQ1) were chosen as the fluorophore / quencher pair for the substrate. BHQ1 should quench more effectively than dabcyl since its absorption is from 480-580 nm with peak absorption around $534 \mathrm{~nm}$, which is much closer to fluorescein's peak emission around $520 \mathrm{~nm}$.

\subsubsection{Enzyme Activity Assay}

The enzyme activity of VT was tested at $30{ }^{\circ} \mathrm{C}$ in $\mathrm{EAB}$ using the polyA substrate 
with fluorescence readings for 40 minutes before and 40 minutes after addition of $\mathrm{N}, \mathrm{N}^{\prime}$ dimethyl-ethylene-diamine. The only fluorescence value that significantly changed during the first incubation was that of the DNase solution (Figure 52). For DNase the substrate was being cleaved separating the fluorescein and BHQ1, causing increased fluorescence signal. This was not observed for the VT samples. This suggests that no large cleavage or rearrangement was occurring to polyA in the presence of VT, although cleavage of adenine residues may have occurred. Each VT solution had a signal higher than that of polyA. VT on its own in buffer did not have a signal different than that of buffer (not shown). As such, this enhanced signal of polyA in the presence of VT was likely some sort of destabilization of the substrate due to interaction with proteins in solution and this effect was greater for VT1 than VT2.

Upon addition of $\mathrm{N}, \mathrm{N}^{\prime}$-dimethyl-ethylene-diamine the signal in all polyA solutions increased. This increase was not observed for samples containing amine alone or with VT which maintained values around blank (not shown). As such, the increase in signal was not due merely to fluorescence by the amine. This increase suggests some nonspecific cleavage of the DNA substrate. N, $\mathrm{N}^{\prime}$-dimethyl-ethylene-diamine, however, had been previously shown to have minimal non-specific cleavage (McHugh and Knowland, 1995). Additionally, the DNase sample no longer increased in signal and compared to polyA alone decreased slightly. The addition of amine likely interferes with the enzyme activity either by the effect on the $\mathrm{pH}$ of the solution or some interaction with the DNase. Additionally, polyA was already heavily damaged by DNase so there was little for the amine to cleave. The VT2 fluorescence signal was the only one that increased during 
the second incubation. This suggests that there were some polyA substrates being catalytically cleaved at abasic sites. However, the magnitude of signal increase was minimal. Although the signal when blanked increased $47 \%$ over the second incubation, the signal of VT2 compared to polyA was only about $20 \%$ larger after the second incubation and started about $23 \%$ larger at the beginning of the first incubation, making it very difficult to discern its activity. The signal could potentially be enhanced with longer incubation times; however, this would limit its usefulness in enhancing detection speed. There is a possibility that A subunits from a crude VT solution would have a higher activity than commercial toxin, however, given the small response it is likely to be even more difficult to detect with additional peptides and other chemicals interfering with activity.

A substrate more similar to the SRL loop containing modified adenine residues labeled with either fluorophore or quencher may offer a stronger signal due to higher target affinity, and avoid the need for the addition of amine to catalyze substrate cleavage having directly induced signal. However, modification of adenine may render VT inactive for the substrate. As such, a nucleotide substrate to detect VTEC by VT activity may not be useful. This is further complicated by presence of nucleases produced by VTEC or other bacteria in food samples that could degrade the substrate resulting in false positives. 


\subsection{Conclusions}

Use of VT's enzymatic activity to catalytically remove adenine from nucleotides was proposed as a means of potentially detecting VTEC. A DNA substrate was designed for this purpose and dual labeled with fluorescein and Black Hole Quencher 1 . The activity of VT could only be indirectly detected by addition of a catalytic reagent $\left(\mathrm{N}, \mathrm{N}^{\prime}\right.$ dimethyl-ethylene-diamine) after enzymatic incubation. There was some evidence to suggest that VT2 did have activity on polyA substrate without chemical activation of the A1 subunit. However, signal induced by VT2 was minimal and a non-specific signal was induced by presence of VT making it even more difficult to determine.

Following these observations, a polyA substrate indirectly labeled would not be useful in VTEC detection. Potentially a substrate with modified adenine has the potential for direct activity detection and better sensitivity, but interference with substrate geometry may result in inhibition or loss of activity. This could be further investigated in the future; however, a DNA substrate is unlikely to enhance VTEC detection. 


\subsection{Contributions to knowledge and future studies}

Verotoxins (VTs) are a family of $A B_{5}$ protein toxins and the defining virulence factor of verotoxin-producing Escherichia coli (VTEC), a pathogroup of E. coli causing enteric illness. VTEC cause illness ranging from asymptomatic carriage to severe complications such as hemolytic uraemic syndrome and death. VTEC include a multitude of serotypes with detection methods often targeting only a select few. Methods to detect all strains are frequently highly technical, laborious, expensive, or slow. Aptamers offer the potential for rapid detection of target molecules when modified into a detection system. Modification is simple, synthesis is reliable, detection can be real time, they are highly stable, and other benefits suggest that they could be used in detection of VT.

In Chapter 2 an aptamer sequence for VT that had been published without characterization information (Fan et al., 2008) was modified by addition of complementary nucleotides, and the fluorophore / quencher pair of fluorescein and dabcyl. This produced the hairpin conformation and aptamer beacon properties desired. Optimization of buffer and testing conditions minimized background signal and improved induced signal stability. Fluorescence of the aptamer beacon was significantly induced by complementary DNA sequences of 10 and 15 bases. At $50 \mathrm{nM}$ fluorescence after 60 minutes was significantly higher for samples tested with VT1a, while samples tested with VT2a had slightly lower fluorescence. This confirmed that the aptamer sequence was selected for VT1a and provided proof of concept for further VT aptamer beacon testing. Aptamer beacon tested on TSA growth plates did not cause a visible 
fluorescence even at $50 \mu \mathrm{M}$ concentration. The positive results led to further experimentation with and optimization of the VT aptamer beacon.

Chapter 3 further characterized the VT aptamer beacon and application conditions. Fluorescence of the aptamer beacon was confirmed to stabilize due to specific interaction with VT1, although this also suggested that the bases important in binding VT were not in the hairpin region. Photobleaching of fluorescein was found to play a major role in discerning interaction between the aptamer beacon and VT. This allowed significant differences after 60 minutes to be found for $10 \mathrm{nM}$ solutions of the aptamer beacon in the presence of VT1 as low as $1.25 \mathrm{nM}$. However, high amino acid content found in TSB liquid growth media was also shown to stabilize the fluorescence photobleaching and make the interaction undetectable. Taken together, the aptamer sequence being used in this beacon has selectivity for VT1, but poor signal induction. This aptamer beacon is greatly limited in its usefulness for detection of VTEC and greatly suggests the need for characterization prior to modification. Binding site analysis of the aptamer sequence including primer-binding regions may lead to a more effective aptamer beacon with a shorter sequence length and more functional stem-loop, leading to reliable signal activation. A new SELEX for VT2, the VT type more frequently associated with severe illness, could lead to aptamer beacons useful in detecting higher risk VTEC strains. SELEX conditions could also be modified to select for stronger structure switching, application in complex media, and heat resistance.

In Chapter 4 use of alternative fluorophore / quencher pairs was assessed for their ability to enhance the aptamer beacon's function, especially for use in other 
aptamer beacons in the future. Availability, ease of modification, stability of fluorescence signal, and other factors led to the selection of Texas Red (TR) / Black hole Quencher 2 (BHQ2) and Cyanine 5 (Cy5) / Black Hole Quencher 3 (BHQ3) as desirable pairs to test. TR had an enhanced stability compared to fluorescein and Cy5, but the fluorescence signal was still greatly affected by photobleaching. Both BHQ2 and BHQ3 had enhanced quenching in their aptamer beacons compared to dabcyl. Interaction with VT was not changed and the same trend previously observed was the result. TR was much more visible on TSA growth media compared to fluorescein, while fluorescein was much more visible on $1.5 \%$ agarose growth plates. As such, selection of an optimal fluorophore / quencher pair will depend on the application of the aptamer beacon. For inexpensive labeling fluorescein and Texas Red possess strong fluorescence and Black Hole Quenchers have highly efficient quenching which could be used as part of any beacon.

In Chapter 5 a DNA substrate was designed to detect VT via its enzyme activity. $\mathrm{VT}$ is a polynucleotide $\mathrm{N}$-glycosidase acting to remove adenine from DNA and RNA samples. As an enzyme, this activity is catalytic and if it could be used to produce a detectable signal could lead to detection of VTEC at very low levels. A polyA substrate was designed with fluorescein and Black Hole Quencher 1 for use as a fluorescent beacon. The substrate was tested with VT1a and VT2a with phosphate backbone cleavage catalyzed by addition of $\mathrm{N}, \mathrm{N}^{\prime}$-dimethylethelenediamine. VT2 was observed to have some activity on the substrate albeit very small and difficult to discern from background signal. The prospect of a nucleotide substrate to detect VTEC appears shaky 
at best although a substrate prepared with labeled adenine may be more effective and could be studied in the future.

Overall, the randomized region of the aptamer sequence previously published (Fan et al., 2008) was further characterized as having specificity for VT1. The use of aptamer beacons for detection of VT was shown to be valuable, although the existing beacon requires further sequence characterization to be effective. Modification with Texas Red proved to be a desirable fluorophore and Black Hole Quenchers as effective quenching molecules for beacon systems. A DNA-based substrate was also found not to be useful for the detection of VTEC. Selection of a new VT2 aptamer sequence that could be used in a future aptamer beacon would be highly valuable. 


\section{References}

Acheson, D.W.K., J. Reidl, X. Zhang, G.T. Keusch, J.J. Mekalanos, and M.K. Waldor. 1998. In Vivo Transduction with Shiga Toxin 1-Encoding Phage. Infect. Immun. 66(9): 44964498.

Acheson, D.W.K., R. Moore, S. de Breucker, L. Lincicome, M. Jacewicz, E. Skutelsky, and G.T. Keusch. 1996. Translocation of Shiga Toxin across Polarized Intestinal Cells in Tissue Culture. Infect. Immun. 64(8): 3294-3300.

Agger, M., F. Scheutz, S. Villumsen, K. Mølbak, and A.M. Petersen. 2015. Antibiotic treatment of verocytotoxin-producing Escherichia coli (VTEC) infection: a systematic review and a proposal. J. Antimicrob. Chemother. 70: 2440-2446.

Ake, J.A., S. Jelacic, M.A. Ciol, S.L. Watkins, K.F. Murray, D.L. Christie, E.J. Klein, and P.I. Tarr. 2005. Relative Nephroprotection During Escherichia coli 0157:H7 Infections: Association With Intravenous Volume Expansion. Pediatrics. 115(6): e673-e680.

Andreoli, S.P., H. Trachtman, D.W.K. Acheson, R.L. Siegler, and T.G. Obrig. 2002. Hemolytic uremic syndrome: epidemiology, pathophysiology, and therapy: Proceedings of the American Society of Pediatric Nephrology Educational Symposium, May 2000, Boston, Massachusetts, USA. Pediatr. Nephrol. 17: 293-298. Atalla, H.N., R. Johnson, S. McEwan, R.W. Usborne, and C.L. Gyles. 2000. Use of a Shiga Toxin (Stx)-Enzyme-Linked Immunosorbent Assay and Immunoblot for Detection and Isolation of Stx-Producing Escherichia coli from Naturally Contaminated Beef. J. Food Protect. 63(9): 1167-1172.

Barbieri, L., P. Valbonesi, M. Brigotti, L. Montanaro, F. Stirpe, and S. Sperti. 1998. Shigalike toxin I is a polynucleotide:adenosine glycosidase. Mol. Microbiol. 29(2): 661-669.

Barbieri, L., P. Valbonesi, E. Bonora, P. Gorini, A. Balognesi, and F. Stirpe. 1997. Polynucleotide:adenosine glycosidase activity of ribosome-inactivating proteins: effect on DNA, RNA and poly(A). Nucleic Acids Res. 25(3): 518-522.

Barbieri, L., P. Gorini, P. Valbonesi, P. Castiglioni, and F. Stirpe. 1994. Unexpected activity of saporins. Nature. 372: 624.

Barrett, T.J., M.E. Potter, and N.A. Strockbine. 1990. Evidence for participation of the macrophage in Shiga-like toxin II-induced lethality in mice. Microb. Pathogenesis. 9: 95-103.

Basu, D., X.-P. Li, J.N. Kahn, K.L. May, P.C. Kahn, N.E. Tumer. 2016. The A1 Subunit of Shiga Toxin 2 Has Higher Affinity for Ribosomes and Higher Catalytic Activity than the A1 Subunit of Shiga Toxin 1. Infect. Immun. 84(1): 149-161.

Bavaro, M.F. 2012. E. coli O157:H7 and Other Toxigenic Strains: The Curse of Global Food Distribution. Curr. Gastroenterol. Rep. 14: 317-323.

Bergan, J., A.B.D. Lingelem, R. Simm, T. Skotland, and K. Sandvig. 2012. Shiga Toxins. Toxicon. 60: 1085-1107.

Bettelheim, K.A. 2007. The Non-0157 Shiga-Toxigenic (Verotoxigenic) Escherichia coli; Under-Rated Pathogens. Crit. Rev. Microbiol. 33(1): 67-87.

Beutin, L., A. Miko, G. Krause, K. Pries, S. Haby, K. Steege, and N. Albrecht. 2007. Identification of Human-Pathogenic Strains of Shiga Toxin-Producing Escherichia coli 
from Food by a Combination of Serotyping and Molecular Typing of Shiga Toxin Genes. Appl. Environ. Microbiol. 73(15): 4769-4775.

Beutin, L., G. Krause, S. Zimmermann, S. Kaulfuss, and K. Gleier. 2004. Characterization of Shiga Toxin-Producing Escherichia coli Strains Isolated from Human Patients in Germany over a 3-Year Period. J. Clin. Microbiol. 42(3): 1099-1108.

Beutin, L., Zimmermann, S., and K. Gleier. 2002. Evaluation of the VTEC-Screen "Seiken" test for detection of different types of Shiga toxin (verotoxin)-producing Escherichia coli (STEC) in human stool samples. Diagn. Micr. Infec. Dis. 42: 1-8.

Beutin, L., D. Geier, H. Steinrück, S. Zimmermann, and F. Scheutz. 1993. Prevalence and Some Properties of Verotoxin (Shiga-Like Toxin)-Producing Escherichia coli in Seven Different Species of Healthy Domestic Animals J. Clin. Microbiol. 31(9): 2483-2488.

Beutin, L., M.A. Montenegro, I. Ørskov, J. Prada, S. Zimmermann, and R. Stephan. 1989. Close Association of Verotoxin (Shiga-Like Toxin) Production with Enterohemolysin Production in Strains of Escherichia coli. J. Clin. Microbiol. 27(11): 2559-2564.

Bielaszewska, M., A. Mellmann, W. Zhang, R. Köck, A. Fruth, A. Bauwens, G. Peters, and H. Karch. 2011. Characterization of the Escherichia coli strain associated with an outbreak of haemolytic uraemic syndrome in Germany, 2011: a microbiological study. Lancet Infect. Dis. 11: 671-676.

Bielaszewska, M., A.W. Friedrich, T. Aldick, R. Schürk-Bulgrin, and H. Karch. 2006. Shiga Toxin Activatable by Intestinal Mucus in Escherichia coli Isolated from Humans: Predictor for a Severe Clinical Outcome. Clin. Infect. Dis. 43: 1160-1167.

Bitzan, M., R. Poole, M. Mehran, E. Sicard, C. Brockus, C. Thuning-Roberson, and M. Rivière. 2009. Safety and Pharmacokinetics of Chimeric Anti-Shiga Toxin 1 and AntiShiga Toxin 2 Monoclonal Antibodies in Healthy Volunteers. Antimicrob. Agents Ch.. 53(7): 3081-3087.

Bitzan, M. 2009. Treatment options for HUS secondary to Escherichia coli O157:H7. Kidney Int. 75(Suppl 112): 562-566.

Blanco, J.E., M. Blanco, M.P. Alonso, A. Mora, G. Dahbi, M.A. Coira, and J. Blanco. 2004a. Serotypes, Virulence Genes, and Intimin Types of Shiga Toxin (Verotoxin)-Producing Escherichia coli Isolates from Human Patients: Prevalence in Lugo, Spain, from 1992 through 1999. J. Clin. Microbiol. 42(1): 311-319.

Blanco, M., J.E. Blanco, A. Mora, G. Dahbi, M.P. Alonso, E.A. González, M.I. Bernárdez and J. Blanco. 2004b. Serotypes, Virulence Genes, and Intimin Types of Shiga Toxin (Verotoxin)-Producing Escherichia coli Isolates from Cattle in Spain and Identification of a New Intimin Variant Gene (eae-६). J. Clin. Microbiol. 42(2): 645651.

Boerlin, P., S.A. McEwen, F. Boerlin-Petzold, J.B. Wilson, R.P. Johnson, and C.L. Gyles. 1999. Associations between Virulence Factors of Shiga Toxin-Producing Escherichia coli and Disease in Humans. J. Clin. Microbiol. 37(3): 497-503.

Bompiani, K.M., R.S. Woodruff, R.C. Becker, S.M. Nimjee, and B.A. Sullenger. 2012. Antidote control of aptamer therapeutics: the road to a safer class of drug agents. Curr. Pharm. Biotechnol. 13(10): 1924-1934.

Bosilevac, J.M. and M. Koohmaraie. 2011. Prevalence and Characterization of Non-O157 Shiga Toxin-Producing Escherichia coli Isolates from Commercial Ground Beef in the 
United States. Appl. Environ. Microbiol. 77(6): 2103-2112.

Brandelli, J.R., T.P. Griener, A. Laing, G. Mulvey, and G.D. Armstrong. 2015. The Effects

of Shiga Toxin 1, 2 and Their Subunits on Cytokine and Chemokine Expression by

Human Macrophage-Like THP-1 Cells. Toxins. 7: 4054-4066.

Brigotti, M., D. Carnicelli, V. Arfilli, N. Tamassia, F. Borsetti, E. Fabbri, P.L. Tazzari, F.

Ricci, P. Pagliaro, E. Spisni, and M.A. Cassatella. 2013. Identification of TLR4 and the

Receptor That Recognizes Shiga Toxins in Human Neutrophils. J. Immunol. 191:

4748-4758.

Brigotti, M., R. Alfieri, P. Sestili, M. Bonelli, P.G. Petronini, A. Guidarelli, L. Barbieri, F.

Stirpe, and S. Sperti. 2002. Damage to nuclear DNA induced by Shiga toxin 1 and

ricin in human endothelial cells. FASEB J. 16(3): 365-72.

Brigotti, M., P. Accorsi, D. Carnicelli, S. Rizzi, A.G. Vara, L. Montanaro, and S. Sperti.

2001. Shiga toxin 1: damage to DNA in vitro. Toxicon. 39: 341-348.

Brigotti, M., D. Carnicelli, P. Alvergna, R. Mazzaracchio, S. Sperti, and L. Montanaro.

1997. The RNA-N-Glycosidase Activity of Shiga-Like Toxin I: Kinetic Parameters of

the Native and Activated Toxin. Toxicon. 35(9): 1431-1437.

Bruce-Grey-Owen Sound Health Unit. 2000. Waterborne Outbreak of Gastroenteritis

Associated with a Contaminated Municipal Water Supply, Walkerton, Ontario, May-

June 2000. Canada Communicable Disease Report. 26-20: 3 pgs.

Bruno, J.G., T. Phillips, A.M. Richarte, T. Montez, A. Garcia, and J.C. Sivils. 2016.

Fluorescent DNA Aptamer-Magnetic Bead Sandwich Assays and Portable

Fluorometer for Sensitive and Rapid Foodborne Pathogen Detection and

Epidemiology. J. Infect. Dis. Epidemiol. 2(2): 6 pgs.

Bruno, J.G., and J.C. Sivils. 2016. Aptamer "Western" Blotting for E. coli Outer

Membrane Proteins and Key Virulence Factors in Pathogenic E. coli Serotypes.

Aptamers and Synthetic Antibodies. 2(1): 29-35.

Bruno, J.G. 2015. Predicting the Uncertain Future of Aptamer-Based Diagnostics and

Therapeutics. Molecules. 20: 6866-6887.

Calderwood, S.B. and J.J. Mekalanos. 1987. Iron Regulation of Shiga-Like Toxin

Expression in Escherichia coli Mediated by the fur Locus. J. Bacteriol. 169(10): 47594764.

Catford, A., V. Kouamé, A. Martinez-Perez, A. Gill, E. Buenaventura, H. Couture, and J.M. Farber. 2014. Risk Profile on Non-O157 Verotoxin-Producing Escherichia Coli in

Produce, Beef, Milk and Dairy Products in Canada. Int. Food Risk Anal. J. 4:21.

Centers for Disease Control and Prevention (CDC). 2014. Surveillance for Foodborne

Disease Outbreaks, United States, 2012: Annual Report. Atlanta, Georgia: US

Department of Health and Human Services, CDC.

Challa, S., S. Tzipori, and A. Sheoran. 2014. Selective Evolution of Ligands by Exponential Enrichment to Identify RNA Aptamers against Shiga Toxins. J. Nucleic Acids. 2014: ID 214929, 8 pages.

Chan, Y.-L., Y. Endo, and I.G. Wool. 1983. The Sequence of the Nucleotides at the $a$ Sarcin Cleavage Site in Rat 28 S Ribosomal Ribonucleic Acid. J. Biol. Chem. 258(21): 12768-12770.

Chang, A.L., M. McKeague, J.C. Liang, and C.D. Smolke. 2014. Kinetic and Equilibrium 
Binding Characterization of Aptamers to Small Molecules using a Label-Free, Sensitive, and Scalable Platform. Anal. Chem. 86: 3273-3278.

Chen, H., S.S. Ahsan, M.B. Santiago-Berrios, H.D. Abruña, and W.W. Webb. 2010. Mechanisms of Quenching of Alexa Fluorophores by Natural Amino Acids. J. Am. Chem. Soc. 132: 7244-7245.

Chui, H., M. Chan, D. Hernandez, P. Chong, S. McCorrister, A. Robinson, M. Walker, L.A.M. Peterson, S. Ratnam, D.J.M. Haldene, S. Bekal, J. Wylie, L. Chui, G. Westmacott, B. Xu, M. Drebot, C. Nadon, J.D. Knox, G. Wang, and K. Cheng. 2015. Rapid, Sensitive, and Specific Escherichia coli H Antigen Typing by Matrix-Assisted Laser Desorption Ionization-Time of Flight-Based Peptide Mass Fingerprinting. J. Clin. Microbiol. 53(8): 2480-2485.

Chui, L., M.-C. Lee, K. Malejczyk, L. Lim, D. Fok, and P. Kwong. 2011. Prevalence of Shiga Toxin-Producing Escherichia coli as Detected by Enzyme-Linked Immunoassays and Real-Time PCR during the Summer Months in Northern Alberta, Canada. J. Clin. Microbiol. 49(12): 4307-4310.

Clementi, N., A. Chirkova, B. Puffer, R. Micura, and N. Polacek. 2010. Atomic mutagenesis reveals A2660 of 235 ribosomal RNA as key to EF-G GTPase activation. Nat. Chem. Biol. 6: 344-351.

Conrady, D.G., M.J. Flagler, D.R. Friedmann, B.D. Vander Wielen, R.A. Kovall, A.A. Weiss, and A.B. Herr. 2010. Molecular Basis of Differential B-Pentamer Stability of Shiga Toxins 1 and 2. PLoS One. 5(12): 9 pgs.

Cornick, N.A., A.F. Helgerson, V. Mai, J.M. Ritchie, and D.W.K. Acheson. 2006. In Vivo Transduction of an Stx-Encoding Phage in Ruminants. Appl. Environ. Microbiol. 72(7): 5086-5088.

Correll, C.C., A. Munishkin, Y.-L. Chan, Z. Ren, I.G. Wool, and T.A. Steitz. 1998. Crystal structure of the ribosomal RNA domain essential for bidding elongation factors. Proc. Natl. Acad. Sci. USA. 95: 13436-13441.

Couturier, M.R., B. Lee, N. Zelyas, and L. Chui. 2011. Shiga-Toxigenic Escherichia coli Detection in Stool Samples Screened for Viral Gastroenteritis in Alberta, Canada. J. Clin. Microbiol. 49(2): 574-578.

Croxen, M.A., Law, R.J., Scholz, R., Keeney, K.M., Wlodarska, M., and Finlay, B.B. 2013. Recent advances in understanding enteric pathogenic Escherichia coli. Clin. Microbiol. Rev. 26(4):822-880.

D’Alessio, L., A. Pinto, A. Cangelosi, P.A. Geoghegan, C. Tironi-Farinati, G.J. Brener, and J. Goldstein. 2016. Sub-Lethal Dose of Shiga Toxin 2 from Enterohemorrhagic Escherichia coli Affects Balance and Cerebellar Cytoarchitecture. Front. Microbiol. 7: 133. doi: 10.3389/fmicb.2016.00133.

de Sablet, T., Y. Bertin, M. Vareille, J.-P. Girardeau, A. Garrivier, A.P. Gobert, and C. Martin. 2008. Differential expression of $s t x_{2}$ variants in Shiga toxin-producing Escherichia coli belonging to seropathotypes A and C. Microbiology. 154: 176-186.

DebRoy, C., P.M. Fratamico, X. Yan, G. Baranzoni, Y. Liu, D.S. Needleman, R. Tebbs, C.D. O’Connell, A. Allred, M. Swimley, M. Mwangi, V. Kapur, J.A. Raygoza Garay, E.L. Roberts, and R. Katani. 2016. Comparison of O-Antigen Gene Clusters of All OSerogroups of Escherichia coli and Proposal for Adopting a New Nomenclature for 
O-Typing. PLOS ONE. 11(1): e0147434. 13 pages.

DebRoy, C., E. Roberts, and P.M. Fratamico. 2011. Detection of O antigens in Escherichia coli. Anim. Health Res. Rev. 12(2): 169-185.

Delcenserie, V., G. LaPointe, T. Charaslertrangsi, A. Rabalski, and M.W. Griffiths. 2011. Glucose Decreases Virulence Gene Expression of Escherichia coli 0157:H7. J. Food Protect. 75(4): 748-752.

Dell'Omo, G., S. Morabito, R. Quondam, U Agrimi, F. Ciuchini, A Macrì, and A. Caprioli. 1998. Feral pigeons as a source of verocytotoxin-producing Escherichia coli. Vet. Rec. 142: 309-310.

Dinu, L.D., and S. Bach. 2011. Induction of Viable but Nonculturable Escherichia coli 0157:H7 in the Phyllosphere of Lettuce: A Food Safety Risk Factor. Appl. Environ. Microbiol. 77(23): 8295-8302.

Dodson, M.L., M.L. Michaels, and R.S. Lloyd. 1994. Unified Catalytic Mechanism for DNA Glycosylases. J. Biol. Chem. 269(52): 32709-32712.

Dowd, S.E., and J.B. Williams. 2008. Comparison of Shiga-Like Toxin II Expression between Two Genetically Diverse Lineages of Escherichia coli 0157:H7. J. Food Protect. 71(8): 1673-1678.

Dubertret, B., M. Calame, and A.J. Libchaber. 2001. Single-mismatch detection using gold-quenched fluorescent oligonucleotides. Nat. Biotechnol. 19: 365-370.

Dwivedi, H.P., R.D. Smiley, L.A. Jaykus. 2013. Selection of DNA aptamers for capture and detection of Salmonella Typhirium using a whole-cell SELEX approach in conjugation with cell sorting. Appl. Microbiol. Biotechnol. 97: 3677-3686.

Endo, Y., A. Glück, Y.-L. Chan, K. Tsurugi, and I.G. Wool. 1990. RN-Protein Interaction: An Analysis With RNA Oligonuleotides Of The Recognition By $\alpha$-Sarcin Of A Ribosomal Domain Critical For Function. J. Biol. Chem. 265(4): 2216-2222.

Endo, Y., and K. Tsurugi. 1988. The RNA N-Glycosidase Activity of Ricin A-chain. J. Biol. Chem. 263(18): 8735-8739.

Endo, Y., Y.-L. Chan, A. Lin, K. Tsurugi, and I.G. Wool. 1988a. The Cytotoxins $\alpha$-Sarcin and Ricin Retain Their Specificity when Tested on a Synthetic Oligoribonucleotide (35Mer) That Mimics a Region of $28 \mathrm{~S}$ Ribosomal Ribonucleic Acid. J. Biol. Chem. 263(17): 7917-7920.

Endo, Y., K, Tsurugi, T. Yutsudo, Y. Takeda, T. Ogasawara, and K. Igarashi. 1988b. Site of action of a Vero toxin (VT2) from Escherichia coli 0157:H7 and of Shiga toxin on eukaryotic ribosomes. Eur. J. Biochem. 171: 45-50.

Ellington, A.D., and J.W. Szostak. 1990. In vitro selection of RNA molecules that bind specific ligands. Nature. 346: 818-822.

Exeni, R.A., G.C. Fernández, and M.S. Palermo. 2007. Role of Polymorphonuclear Leukocytes in the Pathophysiology of Typical Hemolytic Uremic Syndrome. TheScientificWorldJO. 7: 1155-1164.

Famulok, M., and G. Mayer. 2011. Aptamer Modules as Sensors and Detectors. Accounts Chem. Res. 44(12): 1349-1358.

Famulok, M., G. Mayer, and M. Blind. 2000. Nucleic Acid Aptamers--From Selection in Vitro to Applications in Vivo. Accounts Chem. Res. 33: 591-599.

Fan, M., S.R. McBurnett, C.J. Andrews, A.M. Allman, J.G. Bruno, and J.L. Kiel. 2008. 
Aptamer Selection Express: A Novel Method for Rapid Single-Step Selection and Sensing Aptamers. J. Biomol. Tech. 19: 311-321.

Farfan, M.J., and A.G. Torres. 2012. Molecular Mechanisms That Mediate Colonization of Shiga Toxin-Producing Escherichia coli Strains. Infect. Immun. 80(3): 903-913.

Feng, P.C.H., K. Jinneman, F. Scheutz, and S.R. Monday. 2011. Specificity of PCR and Serological Assays in the Detection of Escherichia coli Shiga toxin Subtypes. Appl. Environ. Microbiol. 77(18): 6699-6702.

Fernandez, G.C., M.F. Lopez, S.A. Gomez, M.V. Ramos, L.V. Bentancor, R.J. FernandezBrando, V.I. Landoni, G.I. Dran, R. Meiss, M.A. Isturiz, and M.S. Palermo. 2006. Relevance of neutrophils in the murine model of haemolytic uraemic syndrome: mechanisms involved in Shiga toxin type 2-induced neutrophilia. Clin. Exp. Immunol. 146: 76-84.

Fernández, G.C., M.W.M. Te Loo, T.J.A. van der Velden, L.P.W. van der Heuvel, M.S. Palermo, and L.L.A. Monnens. 2003. Decrease of thrombomodulin contributes to the procoagulant state of endothelium in hemolytic uremic syndrome. Pediatr. Nephrol. 18: 1066-1068.

Frank, C., D. Werber, J.P. Cramer, M. Askar, M. Faber, M. an der Heiden, H. Bernard, A. Fruth, R. Prager, A. Spode, M. Wadl, A. Zoufaly, S. Jordan, M.J. Kemper, P. Follin, L. Müller, L.A. King, B. Rosner, U. Buchgolz, K. Stark, and G. Krause. 2011. Epidemic Profile of Shiga-Toxin-Producing Escherichia coli O104:H4 Outbreak in Germany. New Engl. J. Med. 365: 1771-1780.

Franke, S., D. Harmsen, A. Caprioli, D. Pierard, L.H. Wieler, and H. Karch. 1995. Clonal Relatedness of Shiga-Like Toxin-Producing Escherichia coli 0101 Strains of Human and Porcine Origin. J. Clin. Microbiol. 33(12): 3174-3178.

Franz, E., A.H.A.M. van Hoek, M. Wuite, F.J. van der Wal, A.G. de Boer, E. Bouw, and H.J.M. Aarts. 2015. Molecular Hazard Identification of Non-O157 Shiga ToxinProducing Escherichia coli (STEC). PLoS ONE. 10(3): e0120353.

Fraser, M.E., M.M. Cherney, P. Marcato, G.L. Mulvey, G.D. Armstrong, and M.N.G. James. 2006. Binding of adenine to Stx2, the protein toxin from Escherichia coli 0157:H7. Acta Crystallogr. F62: 627-630.

Fraser, M.E., M. Fujinaga, M.M. Cherney, A.R. Melton-Celsa, E.M. Twiddy, A.D. O'Brien, and M.N.G. James. 2004. Structure of Shiga Toxin Type 2 (Stx2) from Escherichia coli O157:H7. J. Biol. Chem. 279(26): 27511-27517.

Fraser, M.E., M.M. Chernaia, Y.V. Kozlov, and M.N.G. James. 1994. Crystal structure of the holotoxin from Shigella dysenteriae at $2.5 \AA$ A resolution. Nat. Struct. Biol. 1(1): 59-64.

Frenzen, P.D., A. Drake, F.J. Angulo, and The Emerging Infections Program Foodnet Working Group. 2005. Economic Cost of Illness Due to Escherichia coli 0157 Infections in the United States. J. Food Protect. 68(12): 2623-2630.

Friedrich, A.W., J. Borell, M. Bielaszewska, A. Fruth, H. Tschäpe, and H. Karch. 2003. Shiga Toxin 1c-Producing Escherichia coli Strains: Phenotypic and Genetic Characterization and Association with Human Disease. J. Clin. Microbiol. 41(6): 2448-2453.

Friedrich, A.W., M. Bielaszewska, W.-L. Zhang, M. Pulz, T. Kuczius, A. Ammon, and H. 
Karch. 2002, Escherichia coli Harboring Shiga Toxin 2 Gene Variants: Frequency and Association with Clinical Symptoms. J. Infect. Dis. 185: 74-84.

Friesema, I, K. van der Zwaluw, T. Schuurman, M. Kooistra-Smid, E. Franz, Y van Duynhoven, and $W$ van Pelt. 2014. Emergence of Escherichia coli encoding Shiga toxin $2 \mathrm{f}$ in human Shiga toxin-producing E. coli (STEC) infections in the Netherlands, January2008 to December 2011. Euro. Surveill. 19(17): 26-32.

Frydendahl, K. 2002. Prevalence of serogroups and virulence genes in Escherichia coli associated with postweaning diarrhea and edema disease in pigs and a comparison of diagnostic approaches. Vet. Microbiol. 85: 169-182.

Fu, X.J., K. lijima, K. Nozu, K. Hamahira, R. Tanaka, T. Oda, N. Yoshikawa, and M. Matsuo. 2004. Role of p38 MAP kinase pathway in a toxin-induced model of hemolytic uremic syndrome. Pediatr. Nephrol. 19: 844-852.

Fuchs, G., M. Mobassaleh, A. Dnonohue-Rolfe, R.K. Montgomery, R.J. Grand, and G.T. Keusch. 1986. Pathogenesis of Shigella Diarrhea: Rabbit Intestinal Cell Microvillus Membrane Binding Site for Shigella Toxin. Infect. Immun. 53(2): 372-377.

Fuller, C.A., C.A. Pellino, M.J. Flagler, J.E. Strasser, and A.A. Weiss. 2011. Shiga Toxin Subtypes Display Dramatic Differences in Potency. Infect. Immun. 79(3): 1329-1337.

Gamage, S.D., J.E. Strasser, C.L. Chalk, and A.A. Weiss. 2003. Nonpathogenic Escherichia coli Can Contribute to the Production of Shiga Toxin. Infect. Immun. 71(6): 31073115.

Gannon, V.P.J, C. Teerling, S.A. Masri, and C. Gyles. 1990. Molecular cloning and nucleotide sequence of another variant of the Escherichia coli Shiga-like toxin II family. J. Gen. Microbiol. 136: 1125-1135.

Garg, A.X., R.S. Suri, N. Barrowman, F. Rehman, D. Matsell, M.P. Rosas-Arellano, M. Salvadori, R.B. Haynes, and W.F. Clark. 2007. Long-term Renal Prognosis of Diarrhea-Associated Hemolytic Uremic Syndrome: A Systematic Review, Metaanalysis, and Meta-regression. JAMA. 290: 1360-1370.

Garrad, $\varnothing$., B. van Deurs, and K. Sandvig. 1995. Furin-induced Cleavage and Activation of Shiga Toxin. J. Biol. Chem. 270(5): 10817-10821.

Gill, A. and G. Huszczynski. 2016. Enumeration of Escherichia coli 0157:H7 in OutbreakAssociated Beef Patties. J. Food Protect. 79(7): 1266-1268.

Gill, A., and D. Oudit. 2015. Enumeration of Escherichia coli 0157 in OutbreakAssociated Gouda Cheese Made with Raw Milk. J. Food Protect. 78(9): 1733-1737.

Gill, A.O., and C.O. Gill. 2015. Chapter 11: Developments in sampling and test methods for pathogens in fresh meat. In Advances in Microbial Food Safety. (C) 2015 Elsevier Limited. Pages 257-280.

Gill, A., G. Huszczynski, M. Gauthier, and B. Blais. 2014. Evaluation of eight agar media for isolation of shiga toxin-producing Escherichia coli. J. Microbiol. Meth. 96: 6-11.

Gill, A. and C.O. Gill. 2012. Microbial decontamination of raw and ready-to-eat meats in Microbial decontamination in the food industry: novel methods and applications. Copyright Woodhead Publishing Limited, PA, USA. Pages 30-59.

Gill, A., A. Martinez-Perez, S. Mcllwham, and B. Blais. 2012. Development of a Method for the Detection of Verotoxin-Producing Escherichia coli in Food. J. Food. Prot. 75(5): 827-837. 
Glass, K.A., J.M. Loeffelholz, J.P. Ford, and M.P. Doyle. 1992. Fate of Escherichia coli 0157:H7 as Affected by pH or Sodium Chloride and in Fermented, Dry Sausage. Appl. Environ. Microbiol. 58(8): 2513-2516.

Goldwater, P.N., and K.A. Bettelheim. 1996. An Outbreak of Hemolytic Uremic Syndrome due to Escherichia coli 0157:H-: Or Was It? Letters. 2(2): 153-154.

Gonzalo, P., and J.-P. Reboud. 2003. The puzzling lateral flexible stalk of the ribosome. Biol. Cell. 95: 179-193.

Gorski, J.L., I.L. Gonzalez, and R.D. Schmickel. 1987. The Secondary Structure of Human 28S rRNA: The Structure and Evolution of a Mosaic rRNA Gene. J. Mol. Evol. 24: 236251.

Granobles Velandia, C.V., A. Krüger, Y.R. Parma, A.E. Parma, and P.M.A. Lucchesi. 2012. Differences in Shiga toxin and phage production among stx $2 \mathrm{~g}$-positive STEC strains. Front. Cell. Infect. Mi. 2: 5 pages.

Gyles, C.L. 2007. Shiga toxin-producing Escherichia coli: An overview. J. Anim. Sci. 85(E. Suppl.): E45-E62.

Habib, N.F., and M.P. Jackson. 1993. Roles of a Ribosome-Binding Site and mRNA Secondary Structure in Differential Expression of Shiga Toxin Genes. J. Bacteriol. 175(3): 597-603.

Hamaguchi, N., A. Ellington, and M. Stanton. 2001. Aptamer Beacons for the Direct Detection of Proteins. Anal. Biochem. 294: 126-131.

Hamula, C.L.A., J.W. Guthrie, H. Zhang, X.-F. Li, and X.C. Le. 2006. Selection and analytical applications of aptamers. TrAC-Trends Anal. Chem. 25(7): 681-691.

Hancock, D.D., T.E. Besser, D.H. Rice, E.D. Ebel, D.E. Herriott, and L.V. Carpenter. 1998. Multiple sources of Escherichia coli 0157 in feedlots and dairy farms in the Northwestern USA. Prev. Vet. Med. 35: 11-19.

Hathcox, A.K., L.R. Beuchat, and M.P. Doyle. 1995. Death of Enterohemorrhagic Escherichia coli 0157:H7 in Real Mayonnaise and Reduced-Calorie Mayonnaise Dressing as Influenced by Initial Population and Storage Temperature. Appl. Environ. Microbiol. 61(12): 4172-4177.

Henderson, G. 1977. The Effects of Absorption and Self-Absorption Quenching on Fluorescent Intensities. J. Chem. Educ. 54(1): 57-59.

Hermann, T., and D.J. Patel. 2000. Adaptive Recognition by Nucleic Acid Aptamers. Science. 287: 820-825.

Herold, S., H. Karch, and H. Schmidt. 2004. Shiga toxin-encoding bacteriophages genomes in motion. Int. J. Med. Microbiol. 294: 115-121.

Howard, J.G. 1955. Observations on the intoxication produced in mice and rabbits by the neurotoxin of Shigella shigae. Br. J. Exp. Pathol. 36(4): 439-446.

Hull, A.E., D.W.K. Acheson, P. Echeverria, A. Donohue-Rolfe, and G.T. Keusch. 1993. Mitomycin Immunoblot Colony Assay for Detection of Shiga-Like Toxin-Producing Escherichia coli in Fecal Samples: Comparison with DNA Probes. J. Clin. Microbiol. 31(5): 1167-1172.

Hurley, B.P., C.M. Thorpe, and D.W.K. Acheson. 2001. Shiga Toxin Translocation across Intestinal Epithelial Cells Is Enhanced by Neutrophil Transmigration. Infect. Immun. 69(10): 6148-6155. 
Hwang, J.Y., S.T. Kim, H.-S. Han, K. Kim, and J.S. Han. 2016. Optical Aptamer Probes of Fluorescent Imaging to Rapid Monitoring of Circulating Tumor Cell. Sensors. 1909: 13 pgs.

Ichinohe, N., Y. Ohara-Nemoto, T.K. Nemoto, S. Kimura, and S. Ichinohe. 2009. Effects of fosfomycin on Shiga toxin-producing Escherichia coli: quantification of copy numbers of Shiga toxin-encoding genes and their expression levels using real-time PCR. J. Med. Microbiol. 58(7): 971-973.

Igarashi, K., T. Ogasawara, K. Ito, T. Yutsudo, and Y. Takeda. 1987. Inhibition of elongation factor 1-dependent aminoacyl-tRNA binding to ribosomes by Shiga-like toxin I (VT1) from Escherichia coli 0157:H7 and by Shiga toxin. FEMS Microbiol. Lett. 44: 91-94.

Jacewicz, M., H. Clausen, E. Nudelman, A. Donohue-Rolfe, and G.T. Keusch. 1986. Pathogenesis of Shigella Diarrhea: XI. Isolation of a Shigella Toxin-Binding Glycolipid from Rabbit Jejunum and HeLa Cells and Its Identification as Globotriaosylceramide. J. Exp. Med. 163: 1391-1404.

Jackson, M.P., R.J. Neill, A.D. O’Brien, R.K. Holmes, and J.W. Newland. 1987. Nucleotide sequence analysis and comparison of the structural genes for Shiga-like toxin I and Shiga-like toxin II encoded by bacteriophages from Escherichia coli 933. FEMS Microbiol. Lett. 44: 109-114.

James, C.E., K.N. Stanley, H.E. Allison, H.J. Flint, C.S. Stewart, R.J. Sharp, J.R. Saunders, and A.J. McCarthy. 2001. Lytic and Lysogenic Infection of Diverse Escherichia coli and Shigella Strains with a Verocytotoxigenic Bacteriophage. Appl. Environ. Microbiol. 67(9): 4335-4337.

Jayasena, S.D. 1999. Aptamers: An Emerging Class of Molecules That Rival Antibodies in Diagnostics. Clin. Chem. 45(9): 1628-1650.

Jenison, R.D., S.C. Gill, A. Pardi, and B. Polisky. 1994. High-Resolution Molecular Discrimination by RNA. Science. 263: 1425-1429.

Jensen, C., P. Schiellerup, K.E.P. Olsen, F. Scheutz, E. Petersen, P. Gerner-Smidt, and K. Mølbak. 2005. Antimicrobial treatment of asymptomatic carriers of verocytotoxinproducing Escherichia coli: An empiric study. Scand. J. Infect. Dis. 37: 61-63.

Jin, D., H. Qi, S. Chen, T. Zeng, Q. Liu, and S. Wang. 2008. Simultaneous detection of six human diarrheal pathogens by using DNA microarray combined with tyramide signal amplification. J. Microbiol. Meth. 75: 365-368.

Joensen, K.G., F. Scheutz, O. Lund, H. Hasman, R.S. Kaas, E.M. Nielsen, and F.M. Aarestrup. 2014. Real-Time Whole-Genome Sequencing for Routine Typing, Surveillance, and Outbreak Detection of Verotoxigenic Escherichia coli. J. Clin. Microbiol. 52(5): 1501-1510.

Johannes, L., and W. Römer. 2010. Shiga toxins - from cell biology to biomedical applications. Nat. Rev. 8: 105-116.

Johnson, K.E., C.M. Thorpe, and C.L. Sears. 2006. The Emerging Importance of Non-O157 Shiga Toxin-Producing Escherichia coli. Emerging Infections. 43: 1587-1595.

Karch, H., P.I. Tarr, and M. Bielaszewska. 2005. Enterohaemorrhagic Escherichia coli in human medicine. Int. J. Med. Microbiol. 295: 405-418.

Karch, H., H. Schmidt, C. Janetzki-Mittmann, J. Scheef, and M. Kröger. 1999. Shiga toxins 
even when different are encoded at identical positions in the genomes of related temperate bacteriophages. Mol. Gen. Genet. 262: 600-607.

Karve, S.S., and A.A. Weiss. 2014. Glycolipid Binding Preferences of Shiga Toxin Variants. PLOS ONE. 9(7): 10 pages

Karmali, M.A., M. Petric, B.T. Steele, and C. Lim. 1983. Sporadic Cases of HaemolyticUraemic Syndrome Associated With Faecal Cytotoxin And Cytotoxin-Producing Escherichia Coli In Stools. Lancet. 1983: 619-620.

Karpman, D., and A. Ståhl. 2014. Enterohemorrhagic Escherichia coli Pathogenesis and the Host Response. Microbiol. Spectr. 2(5): 15 pages.

Kauffmann, F. 1947. The Serology of the Coli Group. J. Immunol. 57: 71-100.

Kawano, K., H. Ono, O. Iwashita, M. Kurogi, T. Haga, K. Maeda, and Y. Goto. 2012. Relationship between stx Genotype and Stx2 Expression Level in Shiga ToxinProducing Escherichia coli 0157 Strains. Jpn. J. Infect. Dis. 65: 322-325.

Kiel, J.L., J.E. Parker, E.A. Holwitt, and J. Vivekananda. 2004a. DNA Capture Elements for Rapid Detection and Identification of Biological Agents. Proc. SPIE - Int. Soc. Opt. Eng. 5416: 105-110, 2004a.

Kiel, J.L. E.A. Holwitt, J.E. Parker, J. Vivekananda, and V. Franz. 2004b. Nanoparticlelabeled DNA capture elements for detection and identification of biological agents. Proc. SPIE - Int. Soc. Opt. Eng. 5617: 382-387.

Kitov, P.I., J.M. Sadowska, G. Mulvey, G.D. Armstrong, H. Ling, N.S. Pannu, R.J. Read, and D.R. Bundle. 2000. Shiga-like toxins are neutralized by tailored multivalent carbohydrate ligands. Lett. Nat. 403: 669-672.

Konowalchuk, J., J.I. Spiers, and S. Stavric. 1977. Vero Response to a Cytotoxin of Escherichia coli. Infect. Immun. 18(3): 775-779.

Kovbasnjuk, O., M. Edidin, and M. Donowitz. 2001. Role of lipid rafts in Shiga toxin 1 interaction with the apical surface of Caco-2 cells. J. Cell Sci. 114: 4025-4031.

LaPointe, P., X. Wei, and J. Gariépy. 2005. A Role for the Protease-sensitive Loop Region of Shiga-like Toxin 1 in the Retrotranslocation of Its $A_{1}$ Domain from the Endoplasmic Reticulum Lumen. J. Biol. Chem. 280(24): 23310-23318.

Larkey, N.E., L. Zhang, S.S. Lansing, V. Tran, V.L. Seewaldt, and S.M. Burrows. 2016. Förster resonance energy transfer to impart signal-on and -off capabilities in a single microRNA biosensor. Analyst. DOI: 10.1039/c6an01555g

Law, D, and J. Kelly. 1995. Use of Heme and Hemoglobin by Escherichia coli 0157 and Other Shiga-like-Toxin-Producing E. coli Serogroups. Infect. Immun. 63(2): 700-702.

LeClerc, J.E., B. Li, W.L. Payne, and T.A. Cebula. 1996. High Mutation Frequencies Among Escherichia coli and Salmonella Pathogens. Science. 274: 1208-1211.

Leung, P.H.M., J.S.M. Peiris, W.W.S. Ng, R.M. Robins-Browne, K.A. Bettelheim, and W.C. Yam. 2003. A Newly Discovered Verotoxin Variant, VT2g, Produced by Bovine Verocytotoxigenic Escherichia coli. Appl. Environ. Microbiol. 69(12): 7549-7553.

Levine, M.M. 1987. Escherichia coli that Cause Diarrhea: Enterotoxigenic, Enteropathogenic, Enteroinvasive, Enterohemorrhagic, and Enteroadherent. J. Infect. Dis. 155(3): 377-389.

Lindgren, S.W., J.E. Samuel, C.K. Schmitt, and A.D. O'Brien. 1994. The Specific Activities of Shiga-Like Toxin Type II (SLT-II) and SLT-II-Related Toxins of Enterohemorrhagic 
Escherichia coli Differ When Measured by Vero Cell Cytotoxicity but Not by Mouse Lethality. Infect. Immun. 62(2): 623-631.

Lindgren, S.W., A.R. Melton, and A.D. O'Brien. 1993. Virulence of Enterohemorrhagic Escherichia coli 091:H21 Clinical Isolates in an Orally Infected Mouse Model. Infect. Immun. 61(9): 3832-3842.

Ling, H., N.S. Pannu, A. Boodhoo, G.D. Armstrong, C.G. Clark, J.L. Brunton, and R.J. Read. 2000. A mutant Shiga-like toxin Ile bound to its receptor $\mathrm{Gb}_{3}$ : structure of a group II Shiga-like toxin with altered binding specificity. Structure. 8: 253-264.

Ling, H., A. Boodhoo, B. Hazes, M.D. Cummings, G.D. Armstrong, J.L. Brunton, and R.J. Read. 1998. Structure of the Shiga-like Toxin I B-Pentamer Complexed with an Analogue of Its Receptor Gb3. Biochemistry. 37: 1777-1788.

Liu, Y., C. Wang, G. Tyrell, and X.F. Li. 2010. Production of Shiga-like toxins in viable but nonculturable Escherichia coli O1157:H7. Water Research. 44: 711-718.

Liu, W.T., J.H. Wu, E.S.Y. Li, and E.S. Selamat. 2005. Emission Characteristics of Fluorescent Labels with Respect to Temperature Changes and Subsequent Effects on DNA Microchip Studies. Appl. Environ. Microbiol. 71(10): 6453-6457.

Louise, C.B., and T.G. Obrig. 1995. Specific Interaction of Escherichia coli O157:H7Derived Shiga-like Toxin II with Human Renal Endothelial Cells. J. Infect. Dis. 172: 1397-1401.

Louise, C.B., and T.G. Obrig. 1992. Shiga Toxin-Associated Hemolytic Uremic Syndrome: Combined Cytotoxic Effects of Shiga Toxin and Lipopolysaccharide (Endotoxin) on Human Vascular Endothelial Cells In Vitro. Infect. Immun. 60(4): 1536-1543.

Ludwig, K., V. Sarkim, M. Bitzan, M.A. Karmali, C. Bobrowski, H. Ruder, R. Laufs, I. Sobottka, M. Petric, H. Karch, and D.E. Müller-Wiefel. 2002. Shiga Toxin-Producing Escherichia coli Infection and Antibodies against Stx2 and Stx1 in Household Contacts of Children with Enteropathic Hemolytic-Uremic Syndrome. J. Clin. Microbiol. 40(5): 1773-1782.

MacLeod, D.L., and C.L. Gyles. 1989. Effects of culture conditions on yield of Shiga-like toxin-Ilv from Escherichia coli. Can. J. Microbiol. 35: 623-629.

Magnus, T., J. Röther, O. Simova, M. Meier-Cillien, J. Repenthin, F. Möller, J. Gbadamosi, U. Panzer, M. Wengenroth, C. Hagel, S. Kluge, R.K. Stahl, K. Wegscheider, P. Urban, B. Eckert, M. Glatzel, J. Fiehler, and C. Gerloff. 2012. The neurological syndrome in adults during the 2011 northern German E. coli serotype 0104:H4 outbreak. Brain. 135: 1850-1859.

Malyukova, I., K.F. Murray, C. Zhu, E. Boedeker, A. Kane, K. Patterson, J.R. Peterson, M. Donowitz, and O. Kovbasnjuk. 2009. Macropinocytosis in Shiga toxin 1 uptake by human intestinal epithelial cells and transcellular transcytosis. Am. J. Physiol. Gastrointest. Liver. Physiol. 296: G78-G92.

Manandhar, Y., K.C.T. Bahadur, W. Wang, T. Uzawa, T. Aigaki, and Y. Ito. 2015. In vitro selection of a peptide aptamer that changes fluorescence in response to verotoxin. Biotechnol. Lett. 37: 619-625.

March, S.B., and S. Ratnam. 1986. Sorbitol-MacConkey Medium for Detection of Escherichia coli 0157:H7 Associated with Hemorrhagic Colitis. J. Clin. Microbiol. 23(5): 869-872. 
Marras, S.A.E., F.R. Kramer, and S. Tyagi. 2002. Efficiencies of fluorescence resonance energy transfer and contact-mediated quenching in oligonucleotide probes. Nucleic Acids Res. 30(21): e122, 8 pages.

Mauro, S.A., and G.B. Koudelka. 2011. Shiga Toxin: Expression, Distribution, and Its Role in the Environment. Toxins. 3: 608-625.

McDaniel, T.K., K.G. Jarvis, M.S. Donnenberg, and J.B. Kaper. 1995. A genetic locus of enterocyte effacement conserved among diverse enterobacterial pathogens. Proc. Natl. Acad. Sci. 92: 1664-1668.

Mead, P.S. and P.M. Griffin. 1998. Escherichia coli 0157:H7. Lancet. 352: 1207-1212.

Melton-Celsa, A. R. 2014. Shiga toxin (Stx) classification, structure, and function. Microbiology Spectr. 2(3): EHEC-0024-2013. doi: 10.1128/microbiolspec.EHEC-00242013.

Melton-Celsa, A.R., S.C. Darnell, and A.D. O'Brien. 1996. Activation of Shiga-Like Toxins by Mouse and Human Intestinal Mucus Correlates with Virulence of Enterohemorrhagic Escherichia coli 091:H21 Isolates in Orally Infected, Streptomycin-Treated Mice. Infect. Immun. 64(5): 1569-1576.

Moazed, D., J.M. Robertson, and H.F. Noller. 1988. Interaction of elongation factors EF-G and EF-Tu with a conserved loop in 23S RNA. Lett. Nat. 334: 362-364.

Mühldorfer, I., J. Hacker, G.T. Keusch, D.W. Acheson, H. Tschäpe, A.V. Kane, A. Ritter, T. Ölschläger, and A. Donohue-Rolfe. 1996. Regulation of the Shiga-like toxin II operon in Escherichia coli. Infect Immun. 64: 495-502.

Nakano, S., M. Fujimoto, H. Hara, and N. Sugimoto. 1999. Nucleic acid duplex stability: influence of base composition on cation effects. Nucleic Acids Res. 27(14): 29572965.

Nathanson, S., T. Kwon, M. Elmaleh, M. Charbit, E.A. Launay, J. Harambat, M. Brun, B. Ranchin, F. Bandin, S. Cloarec, G. Bourdat-Michel, C. Piètrement, G. Champion, T. Ulinski, and G. Deschênes. 2000. Acute neurological involvement in diarrheaassociated hemolytic uremic syndrome. Clin. J. Am. Soc. Nephrol. 5(7): 1218-1228.

Neely, M.N., and D.I. Friedman. 1998. Functional and genetic analysis of regulatory regions of coliphage $\mathrm{H}-19 \mathrm{~B}$ : location of shiga-like toxin and lysis genes suggest a role for phage functions in toxin release. Mol. Microbiol. 28(6): 1255-1267.

Neupane, M., G.S. Abu-Ali, A. Mitra, D.W. Lacher, S.D. Manning, and J.T. Riordan. 2011. Shiga toxin 2 overexpression in Escherichia coli 0157:H7 strains associated with severe human disease. Microbial Pathogens. 51: 466-470.

Newburg, D.S., P. Chaturvedi, E.L. Lopez, S. Devoto, A. Fayad, and T.G. Cleary. 1993. Susceptibility to Hemolytic-Uremic Syndrome Relates to Erythrocyte Glycosphingolipid Patterns. J. Infect. Dis. 168: 476-479.

Nguyen, Y. and V. Sperandio. 2012. Enterohemorrhagic E. coli (EHEC) pathogenesis. Front. Cell. Infect. Mi. 2: article 90, 7 pages.

Nutiu, R., and Y. Li. 2004. Structure-Switching Signaling Aptamers: Transducing Molecular Recognition into Fluorescence Signaling. Chem. Eur. J. 10: 1868-1876.

Obata, F., K. Tohyama, A.D. Bonev, G.L. Kolling, T.R. Keepers, L.K. Gross, M.T. Nelson, S. Sato, and T.G. Obrig. 2008. Shiga Toxin 2 Affects the Central Nervous System Through Receptor $\mathrm{Gb}_{3}$ Localized to Neurons. J. Infect. Dis. 198(9): 1398-1406. 
Orth, D., K. Grif, A.B. Khan, A. Naim, M.P. Dierich, and R. Würzner. 2007. The Shiga toxin genotype rather than the amount of Shiga toxin or the cytotoxicity of Shiga toxin in vitro correlates with the appearance of the hemolytic uremic syndrome. Diagn. Microbiol. Infect. Dis. 59: 235-242.

Ostroff, S.M., P.I. Tarr, M.A. Neill, J.H. Lewis, N. Hargrett-Bean, and J.M. Kobayashi. 1989. Toxin Genotypes and Plasmid Profiles as Determinants of Systemic Sequelae in Escherichia coli 0157:H7 Infections. J. Infect. Dis. 160(6): 994-998.

Pacheco, A.R., and V. Sperandio. 2012. Shiga toxin in enterohemorrhagic E. coli: regulation and novel anti-virulence strategies. Front. Cell. Infect. Mi. 2(81): 12 pg. Palumbo, S.A., J.E. Call, F.J. Schultz, and A.C. Williams. 1995. Minimum and Maximum Temperatures for Growth and Verotoxin Production by Hemorrhagic Strains of Escherichia coli. J. Food Protect. 58(4): 352-356.

Parry, S.M. and R.L. Salmon. 1998. Sporadic STEC 0157 Infection: Secondary Household Transmission in Wales. Dispatches. 4(4): 657-661.

Paton, A.W., and J.C. Paton. 2002. Direct Detection and Characterization of Shiga Toxigenic Escherichia coli by Multiplex PCR for stx $x_{1}$ st $x_{2}$, eae, ehxA, and saa. J. Clin. Microbiol. 40(1): 271-274.

Paton, A.W., R.M. Ratcliff, R.M. Doyle, J. Seymour-Murray, D. Davos, J.A. Lanser, and J.C. Paton. 1996. Molecular Microbiological Investigation of an Outbreak of HemolyticUremic Syndrome Caused by Dry Fermented Sausage Contaminated with Shiga-Like Toxin-Producing Escherichia coli. J. Clin. Microbiol. 34(7): 1622-1627.

Pellino, C.A., S.S. Karve, S. Pradhan, and A.A. Weiss. 2016. AB ${ }_{5}$ Preassembly Is Not Required for Shiga Toxin Activity. J. Bacteriology. 198(11): 1621-1630.

Piérard, D., G. Muyldermans, L. Moriau, D. Stevens, and S. Lauwers. 1998. Identification of New Verocytotoxin Type 2 Variant B-Subunit Genes in Human and Animal Escherichia coli Isolates. J. Clin. Microbiol. 36(11): 3317-3322.

Pinheiro, V.B., and P. Hollinger. 2014. Towards XNA nanotechnology: new materials from synthetic genetic polymers. Trends Biotechnol. 32(6): 321-328.

Pinheiro, V.B., A.I. Taylor, C. Cozens, M. Abramov, M. Renders, S. Zhang, J.C. Chaput, J. Wengel, S.-Y. Peak-Chew, S.H. McLaughlin, P. Herdewijn, and P. Hollinger. 2012. Synthetic genetic polymers capable of heredity and evolution. Science. 336(6079): 341-344.

Pruimboom-Brees, I.M., T.W. Morgan, M.R. Ackermann, E.D. Nystrom, J.E. Samuel, N.A. Cornick, and H.W. Moon. 2000. Cattle lack the vascular receptors for Escherichia coli 0157:H7 Shiga toxins. Proc. Natl. Acad. Sci. USA. 97(19): 10325-10329.

Rajendran, M., and A.D. Ellington. 2003. In vitro selection of molecular beacons. Nucleic Acids Res. 31(19): 5700-5713.

Reisbig, R., S. Olsnes, and K. Eiklid. 1981. The Cytotoxic Activity of Shigella Toxin. J. Biol. Chem. 256(16): 8739-8744.

Ren, J., I. Utsunomiya, K. Taguchi, T. Ariga, T. Tai, Y. Ihara, and T. Miyatake. 1999. Localization of verotoxin receptors in nervous system. Brain Res. 825: 183-188.

Resch-Genger, U., M. Grabolle, S. Cavaliere-Jaricot, R. Nitschke, and T. Nann. 2008. Quantum dots versus organic dyes as fluorescent labels. Nat. Methods. 5.9: 763775. 
Reyes, A.G., J. Anné, and A. Mejía. 2012. Ribosome-inactivating proteins with an emphasis on bacterial RIPs and their potential medical applications. Future Microbiol. 7(6): 705-717.

Reuter, J. S., \& Mathews, D. H. 2010. RNAstructure: software for RNA secondary structure prediction and analysis. BMC Bioinformatics. 11: 129. (http://rna.urmc.rochester.edu/RNAstructureWeb/index.html)

Richardson, S.E., T.A. Rotman, V. Jay, C.R. Smith, L.E. Becker, M. Petric, N.F. Olivieri, and M.A. Karmali. 1992. Experimental Verocytotoxemia in Rabbits. Infect. Immun. 60(10): 4154-4167.

Riley, L.W., R.S. Remis, S.D. Helgerson, H.B. McGee, J.G. Wells, B.R. Davis, R.J. Hebert, E.S. Olcott, L.M. Johnson, N.T. Hargrett, P.A. Blake, and M.L. Cohen. 1983. Hemorrhagic Colitis Associated With A Rare Escherichia Coli Serotype. New Engl. J. Med. 308(12): 681-685.

Ritchie, J.M., P.L. Wagner, D.W.K. Acheson, and M.K. Waldor. 2003. Comparison of Shiga Toxin Production by Hemolytic-Uremic Syndrome-Associated and Bovine-Associated Shiga Toxin-Producing Escherichia coli Isolates. Appl. Environ. Microbiol. 69(2): 1059-1066.

Rocha, L.B., and R.M.F. Piazza. 2007. Production of Shiga toxin by Shiga toxin-expressing Escherichia coli (STEC) in broth media: from divergence to definition. Lett. Appl. Microbiol. 45: 411-417.

Römer, W., L. Berland, V. Chambon, K. Gaus, B. Windschiegl, D. Tenza, M.R.E. Aly, V. Fraisier, J.-C. Florent, D. Perrais, C. Lamaze, G. Raposo, C. Steinem, P. Sens, P. Bassereau, and L. Johannes. 2007. Shiga toxin induces tubular membrane invaginations for its uptake into cells. Nature. 450: 670-675.

Ruckman, J., L.S. Green, J. Beeson, S. Waugh, W.L. Gillette, D.D. Henninger, L. ClaessonWelsh, and N. Janjić. 1998. 2'-Fluoropyrimidine RNA-based Aptamers to the 165Amino Acid Form of Vascular Endothelial Growth Factor (VEGF165). J. Biol. Chem. 273(32): 20556-20567.

Russo, L.M., A.R. Melton-Celsa, and A.D. O’Brien. 2016. Shiga Toxin (Stx) Type 1a Reduces the Oral Toxicity of Stx Type 2a. J. Infect. Dis. 213: 1271-1279.

Russo, L.M., A.R. Melton-Celsa, M.A. Smith, M.J. Smith, and A.D. O’Brien. 2014. Oral Intoxication of Mice with shiga Toxin Type 2a (Stx2a) and Protection by Anti-Stx2a Monoclonal Antibody 11E10. Infect. Immun. 82(3): 1213-1221.

Rutjes, N.W.P., B.A. Binnington, C.R. Smith, D. Maloney, and C.A. Lingwood. 2002. Differential tissue targeting and pathogenesis of verotoxins 1 and 2 in the mouse animal model. Kidney Int. 62: 832-845.

Saxena, S.K., A.D. O’Brien, and E.J. Ackerman. 1989. Shiga Toxin, Shiga-like Toxin II Variant, and Ricin Are All Single-site RNA N-Glycosidases of 28 S RNA When Microinjected into Xenopus Oocytes. J. Biol. Chem. 264(1): 596-601.

Scallan, E., R.M. Hoekstra, F.J. Angulo, R.V. Tauxe, M.-A. Widdowson, S.L. Roy, J.L. Jones, and P.M. Griffin. 2011. Foodborne Illness Acquired in the United States--Major Pathogens. Emerg. Infect. Dis. 17(1): 7-15.

Scheutz, F. 2014. Taxonomy Meets Public Health: The Case of Shiga Toxin-Producing Escherichia coli. Microbiology Spectr. 2(4): 15 pages. 
Scheutz, F., Teel, L.D., Beutin, L., Piérard, D., Buvens, G., Karch, H., Mellmann, A., Caprioli, A., Tozzoli, R., Morabito, S., Strockbine, N.A., Melton-Celsa, A.R., Sanchez, M., Persson, S., and O'Brien, A.D. 2012. Multicenter evaluation of a sequence-based protocol for subtyping Shiga toxins and standardizing Stx nomenclature. J. Clin. Microbiol. 50(9):2951-2963. doi: 10.1128/JCM.00860-12.

Scheutz, F., and S. Ethelberg. 2008. Meeting Report: Nordic Meeting on detection and surveillance of VTEC infections in humans: Copenhagen 7-8 May 2007. Statens Serum Institute.

Scheutz, F., T. Cheasty, D. Woodward, and H.R. Smith. 2004. Designation of O174 and O175 to temporary $\mathrm{O}$ groups $\mathrm{OX} 3$ and $\mathrm{OX7}$, and six new E. coli $\mathrm{O}$ groups that include Verocytotoxin-producing E. coli (VTEC): 0176, 0177, 0178, 0179, 0180 and 0181. APMIS. 112: 569-584.

Schmidt, H. 2001. Shiga-toxin-converting bacteriophages. Res. Microbiol. 152: 687-695.

Schmidt, H., J. Scheef, S. Morabito, A. Caprioli, L.H. Wieler, and H. Karch. 2000. A New Shiga Toxin 2 Variant (Stx2f) from Escherichia coli Isolated from Pigeons. Appl. Environ. Microbiol. 66(3): 1205-1208.

Schultz, S., D.R. Smith, J.J. Mock, and D.A. Schultz. 2000.Single-target molecule detection with nonbleaching multicolor optical immunolabels. PNAS. 97(3): 669-1001.

Selvin, S.R. 2000. The renaissance of fluorescence resonance energy transfer. Nat. Struct. Biol. 7(9): 730-734.

Seto, E.Y.W., J.A. Soller, and J.M. Colford Jr. 2007. Strategies to Reduce Person-toPerson Transmission during Widespread Escherichia coli 0157:H7 Outbreak. Emerg. Infect. Dis. 13(6): 860-866.

Shi, X., P.K. Khade, K.Y. Sanbonmatsu, and S. Joseph. 2012. Functional Role of the SarcinRicin Loop of the 23S rRNA in the Elongation Cycle of Protein Synthesis. J. Mol. Biol. 419: $125-138$.

Shi, H., X. He, K. Wang, X. Wu, X. Ye, Q. Guo, W. Tan, Z. Qing, X. Yang, and B. Zhou. 2011. Activatable aptamer probe for the contrast-enhanced in vivo cancer imaging based on cell membrane protein-triggered conformation alteration. PNAS. 108(10): 39003905.

Siegler, R., and R. Oakes. 2005. Hemolytic uremic syndrome; pathogenesis, treatment, and outcome. Curr. Opin. Pediatr. 17: 200-204.

Siegler, R.L., T.G. Obrig, T.J. Pysher, V.L. Tesh, N.D. Denkers, and F.B. Taylor. 2003. Response to Shiga toxin 1 and 2 in a baboon model of hemolytic uremic syndrome. Pediatr. Nephrol. 18: 92-96.

Song, S., L. Wang, J. Li, J. Zhao, and C. Fan. 2008. Aptamer-based biosensors. Trends Anal. Chem. 27(2): 108-117.

St. Hilaire, P.M., M.K. Boyd, and E.J. Toone. 1994. Interaction of the Shiga-like Toxin Type 1 B-Subunit with Its Carbohydrate Receptor. Biochemistry. 33: 14452-14463.

Stearns-Kurosawa, D.J., V. Collins, S. Freeman, V.L. Tesh, and S. Kurosawa. 2010. Distinct Physiologic and Inflammatory Response Elicited in Baboons after Challenge with Shiga Toxin Type 1 or 2 from Enterohemorrhagic Escherichia coli. Infect. Immun. 78(6): 2497-2504.

Stephan, R., and F. Untermann. 1999. Virulence Factors and Phenotypical Traits of 
Verotoxin-Producing Escherichia coli Strains Isolated from Asymptomatic Human Carriers. J. Clin. Microbiol. 37(5): 1570-1572.

Stoltenburg, R., C. Reinemann, and B. Strehlitz. 2007. SELEX-A ${ }^{\circledR}$ evolutionary method to generate high-affinity nucleic acid ligands. Biomol. Eng. 24: 381-403.

Strockbine, N.A., Marques, L.R., Newland, J.W., Smith, H.W., Holmes, R.K., and O'Brien,

A.D. 1986. Two toxin-converting phages from Escherichia coli O157:H7 strain 933 encode antigenically distinct toxins with similar biologic activities. Infect. Immun. 53:135-140.

Subils, T., V. Aquili, G. Ebner, and C. Balagué. 2012. Effect of Preservatives on Shiga Toxigenic Phages and Shiga Toxin of Escherichia coli 0157:H7. J. Food Protect. 75(5): 959-965.

Suh, H.S., and L.-A. Jaykus. 2013. Nucleic acid aptamers for capture and detection of Listeria spp. J. Biotechnol. 167: 454-461.

Suh, J.-K., C.J. Hovde, and J.D. Robertus. 1998. Shiga Toxin Attacks Bacterial Ribosomes as Effectively as Eucaryotic Ribosomes. Biochemistry. 37: 9394-9398.

Szewczak, A.A., and P.B. Moore. 1995. The Sarcin/Ricin Loop, a Modular RNA. J. Mol. Biol. 247: 81-98.

Takeshita, S., K. Nakatani, H. Kawase, S. Seki, M, Yamamoto, I. Sekine, and S. Yoshioka. 1999. The Role of Bacterial Lipopolysaccharide-Bound Neutrophils in the Pathogenesis of Kawasaki Disease. J. Infect. Dis. 179: 508-512.

Tam, P.J., and C.A. Lingwood. 2007. Membrane-cytosolic translocation of verotoxin $A_{1}$ subunit in target cells. Microbiology. 153: 2700-2710.

Tarr, P.I., C.A. Gordon, and W.L. Chandler. 2005. Shiga-toxin-producing Escherichia coli and haemolytic uraemic syndrome. Lancet. 365: 1073-1086.

te Loo, D.M.W.M., V.W.M. van Hinsbergh, L.P.W.J. van den Heuvel, and L.A.H. Monnens. 2001. Detection of Verocytotoxin Bound to Circulating Polymorphonuclear Leukocytes of Patients with Hemolytic Uremic Syndrome. J. Am. Soc. Nephrol. 12: 800-806.

te Loo, D.M.W., L.A.H. Monnens, T.J.A.M. van der Velden, M.A. Vermeer, F. Preyers, P.N.M. Demacker, L.P.W.J. van den Heuvel, and V.W.M. van Hinsbergh. 2000. Binding and transfer of verocytotoxin by polymorphonuclear leukocytes in hemolytic uremic syndrome. Blood. 95(11): 3396-3402.

Tesh, V.L., J.A. Burris, J.W. Owens, V.M. Gordon, E.A. Wadolkowski, A.D. O’Brien, and J.E. Samuel. 1993. Comparison of the Relative Toxicities of Shiga-Like Toxins Type I and Type II for Mice. Infect. Immun. 61(8): 3392-3402.

Tesh, V.L., J.E. Samuel, L.P. Perera, J.B. Sharefkin, and A.D. O'Brien. 1991. Evaluation of the Role of Shiga and Shiga-like Toxins in Mediating Direct Damage to Human Vascular Endothelia Cells. J. Infect. Dis. 164: 344-352.

Teunis, P., K. Takumi, and K. Shinagawa. 2004. Dose Response for Infection by Escherichia coli 0157:H7 from Outbreak Data. Risk Analysis. 24(2): 401-407.

Thomas, M.K., R. Murray, L. Flockhart, K. Pintar, F. Pollari, A. Fazil, A. Nesbitt, and B. Marshall. 2013. Estimates of the Burden of Foodborne Illness in Canada for 30 Specified Pathogens and Unspecified Agents, Circa 2006. Foodborne Pathog. Dis. 10(7): 639-648. 
Tilden, J.J., W. Young, A.M. McNamara, C. Custer, B. Boesel, M.A. Lambert-Fair, J. Majkowski, D. Vugia, S.B. Werner, J. Hollingsworth and J.G.J. Morris. 1996. A new route of transmission for Escherichia coli: infection from dry fermented salami. Am. J. Public Health. 86:1142-1145.

Tuerk, C., and L. Gold. 1990. Systematic evolution of ligands by exponential enrichment: RNA ligands to bacteriophage T4 DNA Polymerase. Science. 249: 505-510.

Tumer, N.E., and X.-P. Li. 2012. Interaction of Ricin and Shiga Toxins with Ribosomes. Curr. Top. Microbiol. Immunol. 357: 1-18.

Tuttle, J., Gomez, T., Doyle, M.P., Wells, J.G., Zhao, T., Tauxe, R.V., and Griffin, P.M. 1999. Lessons from a Large Outbreak of Escherichia coli 0157:H7 Infections: Insights into the Infectious Dose and Method of Widespread Contamination of Hamburger Patties. Epidemiol. Infect. 122: 185-192.

Tyagi, S., D.P. Bratu, and F.R. Kramer. 1998. Multicolor molecular beacons for allele discrimination. Nat. Biotechnol. 16: 49-53.

Tyagi, S., and F.R. Kramer. 1996. Molecular Beacons: Probes that Fluoresce upon Hybridization. Nat. Biotechnol. 14: 303-308.

van der Kar, N.C.A.J., L.A.H. Monnens, M.A. Karmali, and V.W.M. van Hinsbergh. 1992. Tumor Necrosis Factor and Interleukin-1 Induce Expression of the Verocytotoxin Receptor Globotriaosylceramide on Human Endothelial Cells: Implications for the Pathogenesis of the Hemolytic Uremic Syndrome. Blood. 80(11): 2755-2764.

Van Dyck, M., and W. Proesmans. 2004. Renoprotection by ACE inhibitors after severe hemolytic uremic syndrome. Pediatr. Nephrol. 19: 688-690.

van Setten, P.A., V.W.M. van Hinsbergh, T.J.A.N. van der Velden, N.C.A.J. van de Kar, M. Vermeer, J.D. Mahan, K.J.M. Assmann, L.P.W.J. van den Heuvel, and L.A.H. Monnens. 1997. Effects of TNF $\alpha$ on verocytotoxin cytotoxicity in purified human glomerular microvascular endothelial cells. Kidney Int. 51: 1245-1256.

Vareille, M., T. de Sablet, T. Hindré, C. Martin, and A.P. Gobert. 2007. Nitric oxide inhibits Shiga-toxin synthesis by enterohemorrhagic Escherichia coli. PNAS. 104(24): 10199-10204.

Varma, J.K., K.D. Greene, M.E. Reller, S.M. DeLong, J. Trottier, S.F. Nowicki, M. DiOrio, E.M. Koch, T.L. Bannerman, S.T. York, M.-A. Lambert-Fair, J.G. Wells, and P.S. Mead. 2003. An Outbreak of Escherichia coli 0157 Infection Following Exposure to a Contaminated Building. JAMA. 290(20): 2709-2712.

Velázquez-Campoy, A., H. Ohtaka, A. Nezami, S. Muzammil, and E. Friere. 2004. Isothermal Titration Calorimetry. Chapter 17.8 in Current Protocols in Cell Biology.

Wadolkowski, E.A., L.M. Sung, J.A. Burris, J.E. Samuel, and A.D. O'Brien. 1990. Acute Renal Tubular Necrosis and Death of Mice Orally Infected with Escherichia coli Strains That Produce Shiga-Like Toxin Type II. Infect. Immun. 58(12): 3959-3965.

Wang, L., D. Rothemund, H. Curd, and P.R. Reeves. 2003. Species-Wide Variation in the Escherichia coli Flagellin (H-Antigen) Gene. J. Bacteriol. 185(9): 2936-2943.

Weeratna, R.D., and M.P. Doyle. 1991. Detection and Production of Verotoxin 1 of Escherichia coli 0157:H7 in Food. Appl. Environ. Microb. 57(10): 2951-2955.

Werber, D., A. Fruth, U. Buchholz, R. Prager, M.H. Kramer, A. Ammon, and H. Tschäpe. 2003. Strong Association Between Shiga Toxin-Producing Escherichia coli 0157 and 
Virulence Genes stx2 and eae as Possible Explanation for Predominance of Serogroup 0157 in Patients with Haemolytic Uraemic Syndrome. Eur. J. Clin. Microbiol. Infect. Dis. 22: 726-730.

World Health Organization (WHO). 2015. WHO estimates of the global burden of foodborne diseases. Geneva, Switzerland: WHO.

Wong, C.S., S. Jelacic, R.L. Habeeb, S.L. Watkins, and P.I. Tarr. 2000. The Risk of the Hemolytic-Uremic Syndrome After Antibiotic Treatment of Escherichia coli O157:H7 Infections. New Engl. J. Med. 342(26): 1930-1936.

Wu, W., S. Zhao, Y. Mao, Z. Fang, X. Lu, and L. Zeng. 2015. A sensitive lateral flow biosensor for Escherichia coli 0157:H7 detection based on aptamer mediated strand displacement amplification. Analytica Chimica Acta. 861: 62-68.

Wu, C., C.J. Yang, and W. Tan. 2013. Chapter 11: Molecular Aptamer Beacons in Molecular Beacons. (C) Springer-Verlag Berlin Heidelberg 2013. DOI 10.1007/978-3642-39109-5 11

Yamamoto, R., and P.K.R. Kumar. 2000. Molecular beacon aptamer fluoresces in the presence of Tat protein of HIV-1. Genes to Cells. 5: 389-396.

Yang, Y., M. Zhou, H. Hou, J. Zhu, F. Yao, X. Zhang, X. Zhu, P.R. Hardwidge, and G. Zhu. 2014. Quorum-sensing gene luxS regulates flagella expression and Shiga-like toxin production in F18ab Escherichia coli. Can. J. Microbiol. 60: 355-361.

You, Y., A.V. Tataurov, and R. Owczarzy. 2011. Measuring Thermodynamic Details of DNA Hybridization Using Fluorescence. Biopolymers. 95(7): 472-486.

Yu, M., and D.B. Haslam. 2005. Shiga Toxin Is Transported from the Endoplasmic Reticulum following Interaction with the Luminal Chaperone HEDJ/ERdj3. Infect. Immun. 73(4): 2524-2532.

Yung, A., W.B. Turnbull, A.P. Kalverda, G.S. Thompson, S.W. Homans, P. Kitov, and D.R. Bundle. 2003. Large-Scale Millisecond Intersubunit Dynamics in the B Subunit Homopentamer of the Toxin Derived from Escherichia coli 0157. J. Am. Chem. Soc. 125: 13058-13062.

Zangari, T., A.R. Melton-Celsa, A. Panda, M.A. Smith, I. Tatarov, L. De Tolla, and A.D. O'Brien. 2014/ Enhanced Virulence of the Escherichia coliO157:H7 SpinachAssociated Outbreak Strain in Two Animal Models Is Associated with Higher Levels of Stx2 Production after Induction with Ciprofloxacin. Infect. Immun. 82(12): 49684977.

Zimbres, F.M., A. Tárnok, H. Ulrich, and C. Wregner. 2013. Aptamers: Novel Molecules as Diagnostic Markers in Bacterial and Viral Infections? BioMed Research International. 2013: Article ID 731516, 7 pages

Zoja, C., D. Corna, C. Farina, G. Sacchi, C. Lingwood, M.P. Doyle, V.V. Padhye, M. Abbate, and G. Remuzzi. 1992. Verotoxin glycolipid receptors determine the localization of macroangiopathic process in rabbits given verotoxin-1. J. Lab. Clin. Med. 120(2): 229-238.

Zuker, M. 2003. Mfold web server for nucleic acid folding and hybridization prediction. Nucleic Acids Res. 31 (13): 3406-3415. (http://mfold.rna.albany.edu/)

Patents: 
RU 2556552 C1. Владимировна, К.А., К.А. Владимирович, Г.В. Павловна, Л.Н.

Михайловна, К.О. Николаевна, Р.А. Константиновна, Ш.И. Георгиевич, and Д.И. Алексеевич. 2015. Последовательность днк-аптамеров, связывающая шигатоксин типа 2. Russian Patent. Oct. 27, 2015.

US 2004/0023265 A1. Vivekananda, J., and J.L. Kiel. 2004. Methods and Compositions for Nucleic Acid Ligands Against Shiga Toxin and/or Shiga-Like Toxin. United States Patent Application Publication. Published Feb. 5, 2004.

US 2006/0008841 A1. Okada, K., S. Senda, A. Kobayashi, E. Fukusaki, T. Honda, I. Yanagihara, and T. Nakanishi. 2006. Aptamer Capable of Specifically Adsorbing to Verotoxin-1 and Method for Obtaining the Aptamer. United States Patent Application Publication. Published Jan. 12, 2006.

US 8,790,877 B2. Holwitt, E.A., J.L. Kiel, and V.F. Sorola. 2014. Using DNA Aptamers and Quantum Dots for the Detection of Proteins or Other Targets. United States Patent. Jul. 29, 2014. 
Appendices 


\section{Appendix A Additional Figures}

\section{List of Figures:}

Figure A-1 - Most probable secondary structure of Fan et al.'s forward (+) verotoxin aptamer sequence predicted with Mfold software; with increasing free energy (dG) of $0.46,0.7,0.83$, and 1.11 from left to right (Fan et al., 2008; Zuker, 2003).

Figure A-2 - Most probable secondary structure for aptamer beacon with 10 complementary bases (A10) (left) and 15 complementary bases (A15) (right) predicted with Mfold software (Zuker, 2003).

Figure A-3 - Fluorescence intensity results of fluorescent control DNA (FC) solutions in TEB buffer showing a linear range of $2.5 \times 10^{-3}$ to $5 \mu \mathrm{M}$ for Synergy 2 plate reader.... 172

Figure A-4 - Fluorescence intensity of fluorescent control DNA (FC) and fluorescein / dabcyl labeled aptamer beacon with 10 complementary bases (A10) at $75 \mathrm{nM}$ in TEB buffer. About 40 minutes passed while heating to $60^{\circ} \mathrm{C}$.

Figure A-5 - Fluorescence intensity of fluorescent control DNA (FC) and fluorescein / dabcyl labeled aptamer beacon with 10 complementary bases (A10) at $5.7 \mathrm{nM}$ in TEB buffer. About 40 minutes passed while heating to $60^{\circ} \mathrm{C}$.

Figure A-6 - Fluorescence intensity effects of refolding in TEB buffer (B) of fluorescein / dabcyl labeled aptamer beacon with 10 complementary bases (A10) (100 nM) alone and in the presence of $\mathrm{C} 10(400 \mathrm{nM})$. Fluorescent control DNA (FC) had a value of about $27 \mathrm{x}$ $10^{4}$ RFUs.

Figure A-7 - Fluorescence intensity effects of refolding in TEB buffer (B) of fluorescein / dabcyl labeled aptamer beacon with 10 complementary bases (A10) (10 nM) alone and in the presence of $\mathrm{C} 10(40 \mathrm{nM})$. Fluorescent control DNA $(\mathrm{FC})$ had a value of about $21 \mathrm{x}$ $10^{3}$ RFUs.

Figure A-8 - The effects of buffer salinity (TEB, 20mM; or TES, $1 \mathrm{M}$ ) and refolding in buffer (B) on background fluorescence of fluorescein / dabcyl labeled aptamer beacon with 10 complementary bases (A10) $(1 \mu \mathrm{M})$. Fluorescent control DNA (FC) values were around $35 \times 10^{5}$ RFUs. Ex. $495 \pm 10$ nm, Em. $528 \pm 20$ nm.

Figure A-9 - The effects of buffer salinity (TEB, 20mM; or TES, $1 \mathrm{M}$ ) and refolding in buffer (B) on background fluorescence of fluorescein / dabcyl labeled aptamer beacon with 10 complementary bases (A10) (100 nM). Fluorescent control DNA (FC) values were around $30 \times 10^{4}$ RFUs. 
Figure A-10 - The effects of buffer salinity (TEB, 20mM; or TES, $1 \mathrm{M}$ ) and refolding in buffer (B) on background fluorescence of fluorescein / dabcyl labeled aptamer beacon with 10 complementary bases (A10) (10 nM). Fluorescent control DNA (FC) values were around $22 \times 10^{3}$ RFUs

Figure A-11 - Fluorescence intensity of fluorescein / dabcyl labeled aptamer beacon with 10 complementary bases (A10) $(1 \mu \mathrm{M})$ in TEB or TES at various $\mathrm{A} 10: \mathrm{C} 10$ ratios after refolding in buffer. Fluorescent control DNA (FC) values were about $34 \times 10^{5}$ RFUs. Ex. $495 \pm 10$ nm, Em. $528 \pm 20$ nm. 176

Figure A-12 - Fluorescence intensity of fluorescein / dabcyl labeled aptamer beacon with 10 complementary bases (A10) $(100 \mathrm{nM})$ in the presence of C10 or C15 (100 nM) in TEB (B) and TES (S). Fluorescent control DNA (FC) values were about $25 \times 10^{4}$ RFUs. .. 176

Figure A-13 - Fluorescence of fluorescein / dabcyl labeled aptamer beacon (FLQ) (10 $\mathrm{nM}$ ) measured over time with varying VT1 concentrations in TES buffer. Fluorescein labeled aptamer (FLX) values (10 nM) were around $17 \times 10^{3}$ RFUs. Data points represent the average of 6 wells from replicates 1 and 2. Error bars shown are SD. Ex. $495 \pm 10 \mathrm{~nm}$, Em. $528 \pm 20 \mathrm{~nm}$.

Figure A-14 - Fluorescence of fluorescein / dabcyl labeled aptamer beacon (FLQ) (10 $\mathrm{nM}$ ) measured over time with varying VT1 concentrations in TES buffer. Fluorescein labeled aptamer (FLX) values (10 nM) were around $17 \times 10^{3}$ RFUs. Data points represent the average of 12 wells over 4 replicates. Error bars represent SD. Ex. $495 \pm 10 \mathrm{~nm}$, Em. $528 \pm 20 \mathrm{~nm}$.

Figure A-15 - Blanked change in fluorescence over time for the Texas Red / Black Hole Quencher 2 labeled aptamer beacon (TRQ) (10 nM) in the presence of VT1a or VT2a (each at $10 \mathrm{nM}$ ). A) Full view including unquenched Texas Red labeled aptamer (TRX) sequence; B) close-up on curves excluding Cyanine 5 labeled aptamer (CYX). Data points are the average of triplicate readings. Error bars show SD. Ex. $596 \pm 15$ nm, Em. $680 \pm 30$ $\mathrm{nm}$

Figure A-16 - Blanked change in fluorescence over time for Cyanine 5 / Black Hole Quencher 3 labeled aptamer beacon (CYQ) (10 nM) in the presence of VT1a or VT2a (each at $10 \mathrm{nM}$ ). A) Full view including unquenched Cyanine 5 labeled aptamer (CYX) sequence; B) close-up on curves excluding CYX. Data points are the average of triplicate readings. Error bars show SD. Ex. $620 \pm 40 \mathrm{~nm}$, Em. $680 \pm 30 \mathrm{~nm}$.

Figure A-17 - Comparison of water and $100 \mu \mathrm{M}$ solutions of fluorescein, Texas Red, and NIR-641 (top to bottom) illuminated by white light, handheld UV lamp, and green LED flashlight (left to right). Images taken by cellphone camera. 179

Figure A-18 - TSA plates used for visible fluorescence comparison in white light. Texas Red (left), both (middle), fluorescein (right). Image taken with a cell phone camera... 179 
Figure A-19 - Comparison of visible fluorescence of fluorescein and Texas Red spotted on a $1.5 \%$ agarose plate. Solutions were between $40-1 \mu \mathrm{M}$. Samples were spotted in triplicate and the plate was refrigerated overnight to allow spots to dry. Image taken with a cell phone camera.

Figure A-20 - Fluorescence values for the first enzyme activity assay for the first 40minute incubation at $30{ }^{\circ} \mathrm{C}$. Solutions contained DNase ( 0.5 units per $\left.100 \mu \mathrm{L}\right)$, VT1 or VT2 $(10 \mathrm{nM})$, in addition to polyA $(3.75 \mu \mathrm{M})$ in EAB. Data points are the average of triplicate. Error bars are SD.

Figure A-21 - Fluorescence values for the first enzyme activity assay for the second 40minute incubation at $30^{\circ} \mathrm{C}$. Solutions contained DNase ( 0.5 units per $\left.100 \mu \mathrm{L}\right)$, VT1 or VT2 (10 nM), crude VT2a (117 nM), in addition to polyA (3.75 $\mu \mathrm{M})$ and N, $\mathrm{N}^{\prime}$-dimethylethylene-diamine $(100 \mathrm{mM})$ in $\mathrm{EAB}$. Data points are the average of triplicate. Error bars are SD.....

Figure A-22 - Fluorescence values for the second enzyme activity assay for the first 40minute incubation at $30^{\circ} \mathrm{C}$. Solutions contained DNase $(0.5$ units per $100 \mu \mathrm{L}), \mathrm{VT} 1$ or VT2 (10 nM), crude VT2a (117 nM), in addition to polyA $(3.75 \mu \mathrm{M})$ in EAB. Data points are the average of triplicate. Error bars are SD

Figure A-23 - Fluorescence values for the second enzyme activity assay for the second 40-minute incubation at $30^{\circ} \mathrm{C}$. Solutions contained DNase ( 0.5 units per $\left.100 \mu \mathrm{L}\right), \mathrm{VT} 1$ or VT2 (10 nM), crude VT2a (117 nM), in addition to polyA $(3.75 \mu \mathrm{M})$ and N, $\mathrm{N}^{\prime}$-dimethylethylene-diamine $(100 \mathrm{mM})$ in $\mathrm{EAB}$. Data points are the average of triplicate. Error bars are SD.

Figure A-24 - Fluorescence values for the third enzyme activity assay for the first 40minute incubation at $30^{\circ} \mathrm{C}$. Solutions contained DNase ( 0.5 units per $\left.100 \mu \mathrm{L}\right)$, VT1 or VT2 or crude VT2a (10 nM), in addition to polyA $(3.75 \mu \mathrm{M})$ in EAB. Data points are the average of duplicate. Error bars are SD.....

Figure A-25 - Fluorescence values for the third enzyme activity assay for the second 40minute incubation at $30^{\circ} \mathrm{C}$. Solutions contained DNase (0.5 units per $\left.100 \mu \mathrm{L}\right), \mathrm{VT} 1$ or VT2 or crude VT2a (10 nM), in addition to polyA $(3.75 \mu \mathrm{M})$ and N,N'-dimethyl-ethylenediamine $(100 \mathrm{mM})$ in EAB. Data points are the average of duplicate. Error bars are SD 


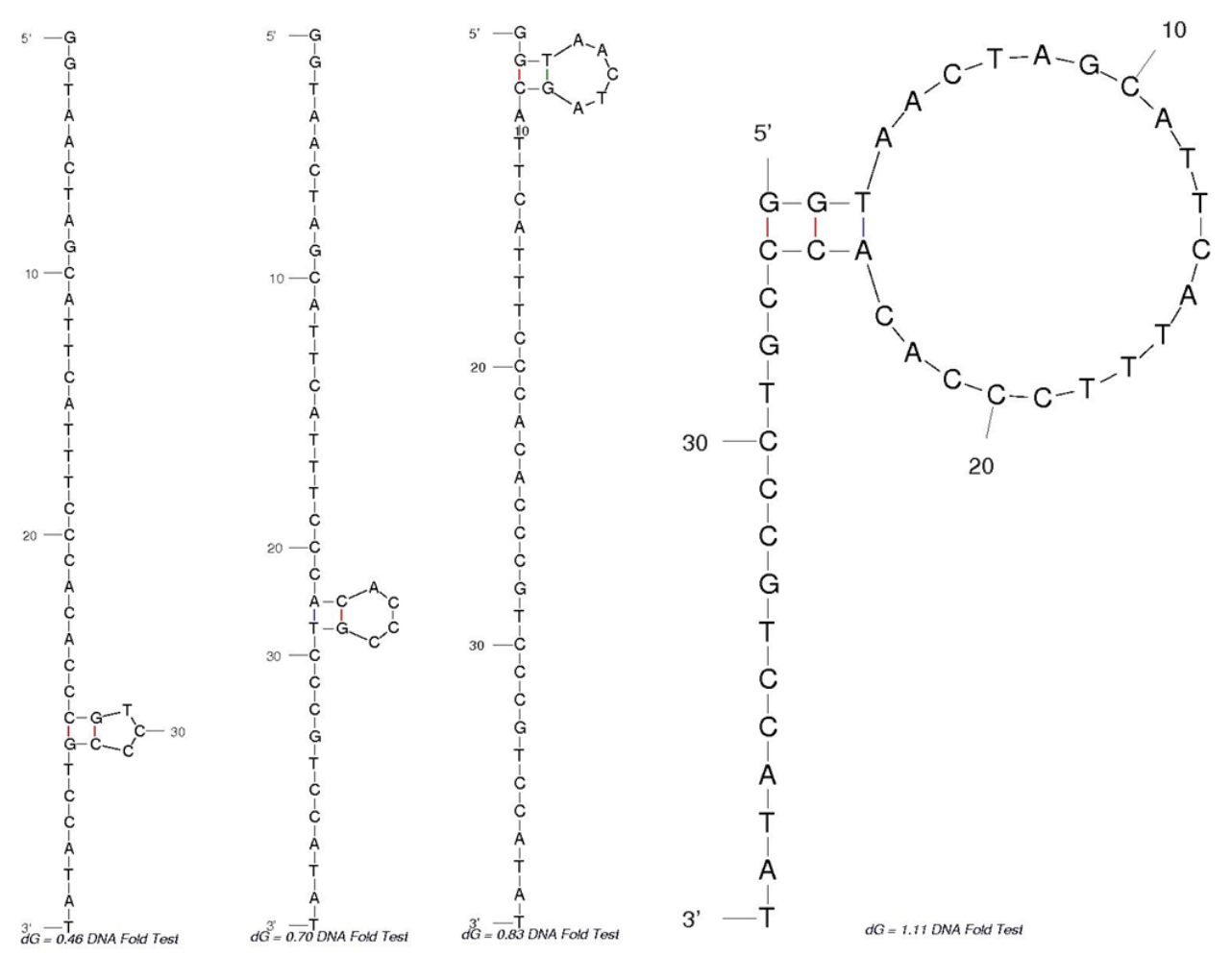

Figure A-1 - Most probable secondary structure of Fan et al.'s forward (+) verotoxin aptamer sequence predicted with Mfold software; with increasing free energy (dG) of $0.46,0.7,0.83$, and 1.11 from left to right (Fan et al., 2008; Zuker, 2003).
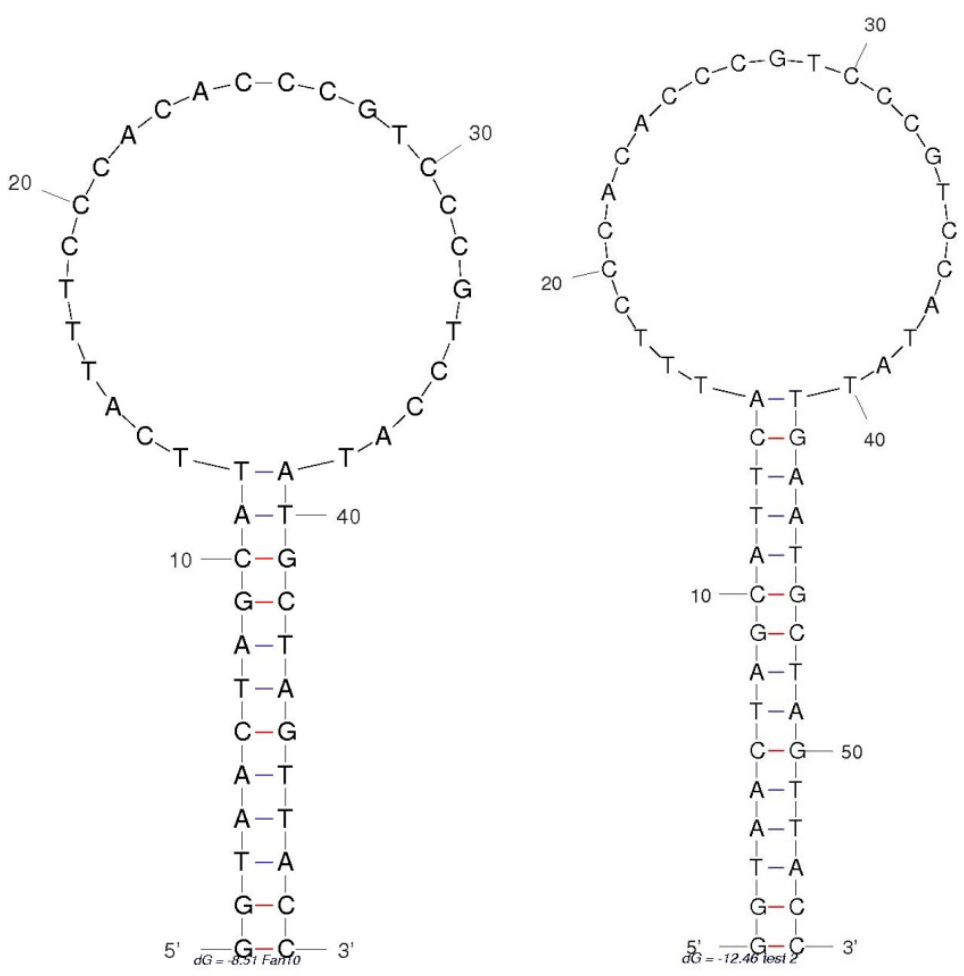

Figure A-2 - Most probable secondary structure for aptamer beacon with 10 complementary bases (A10) (left) and 15 complementary bases (A15) (right) predicted with Mfold software 
(Zuker, 2003).

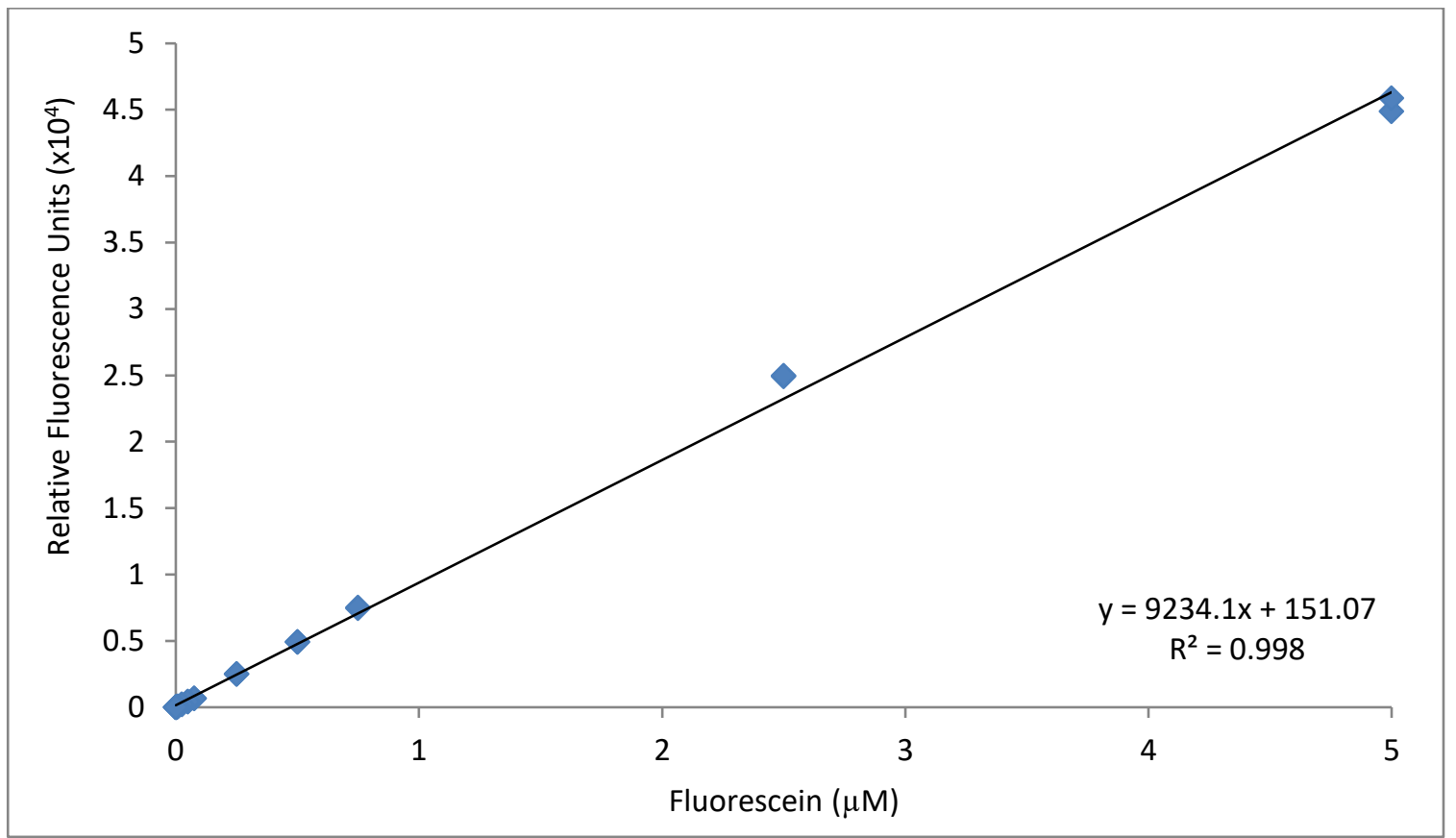

Figure A-3 - Fluorescence intensity results of fluorescent control DNA (FC) solutions in TEB buffer showing a linear range of $2.5 \times 10^{-3}$ to $5 \mu \mathrm{M}$ for Synergy 2 plate reader.

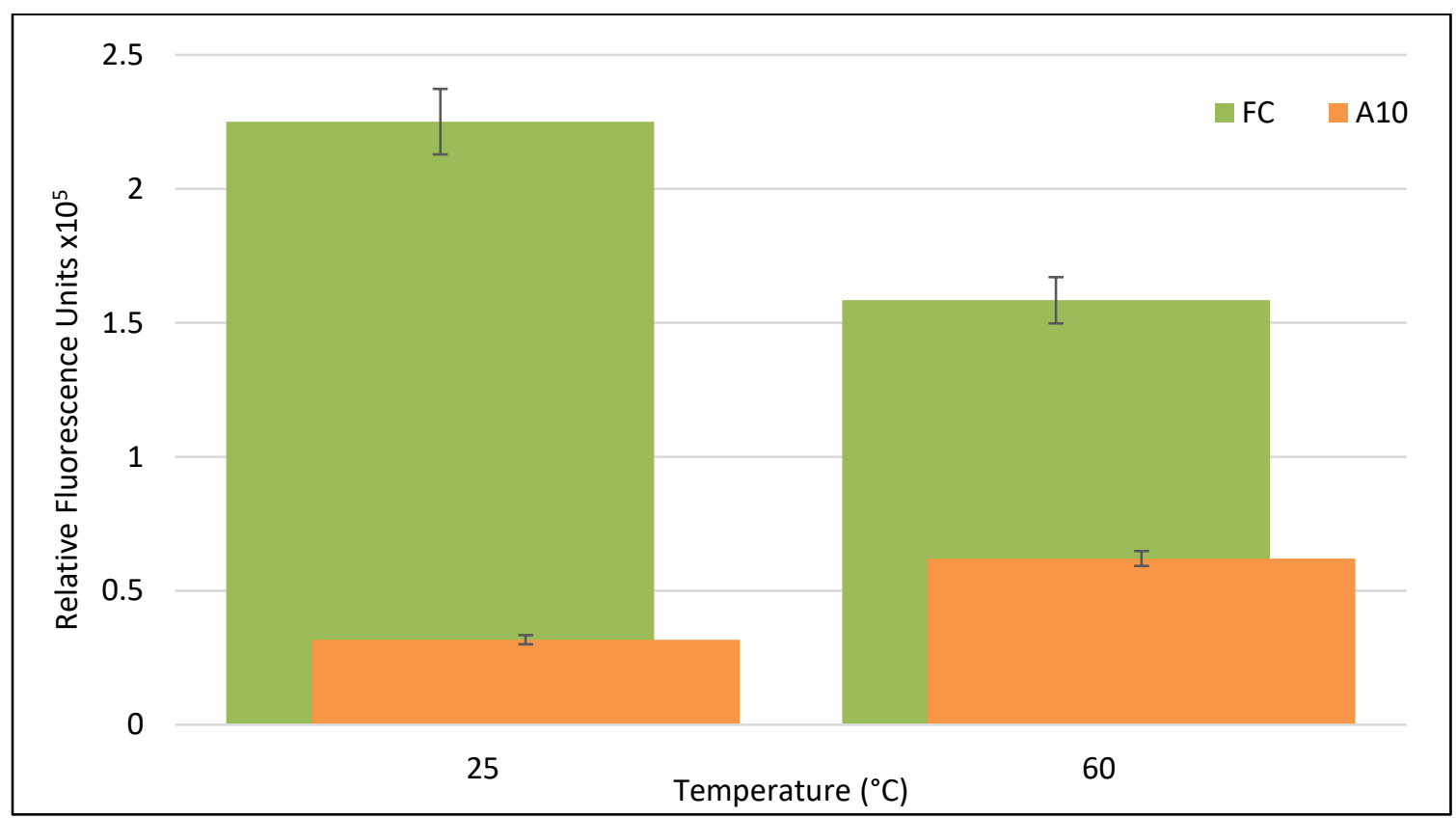

Figure A-4 - Fluorescence intensity of fluorescent control DNA (FC) and fluorescein / dabcyl labeled aptamer beacon with 10 complementary bases (A10) at 75 nM in TEB buffer. About 40 minutes passed while heating to $60^{\circ} \mathrm{C}$. 


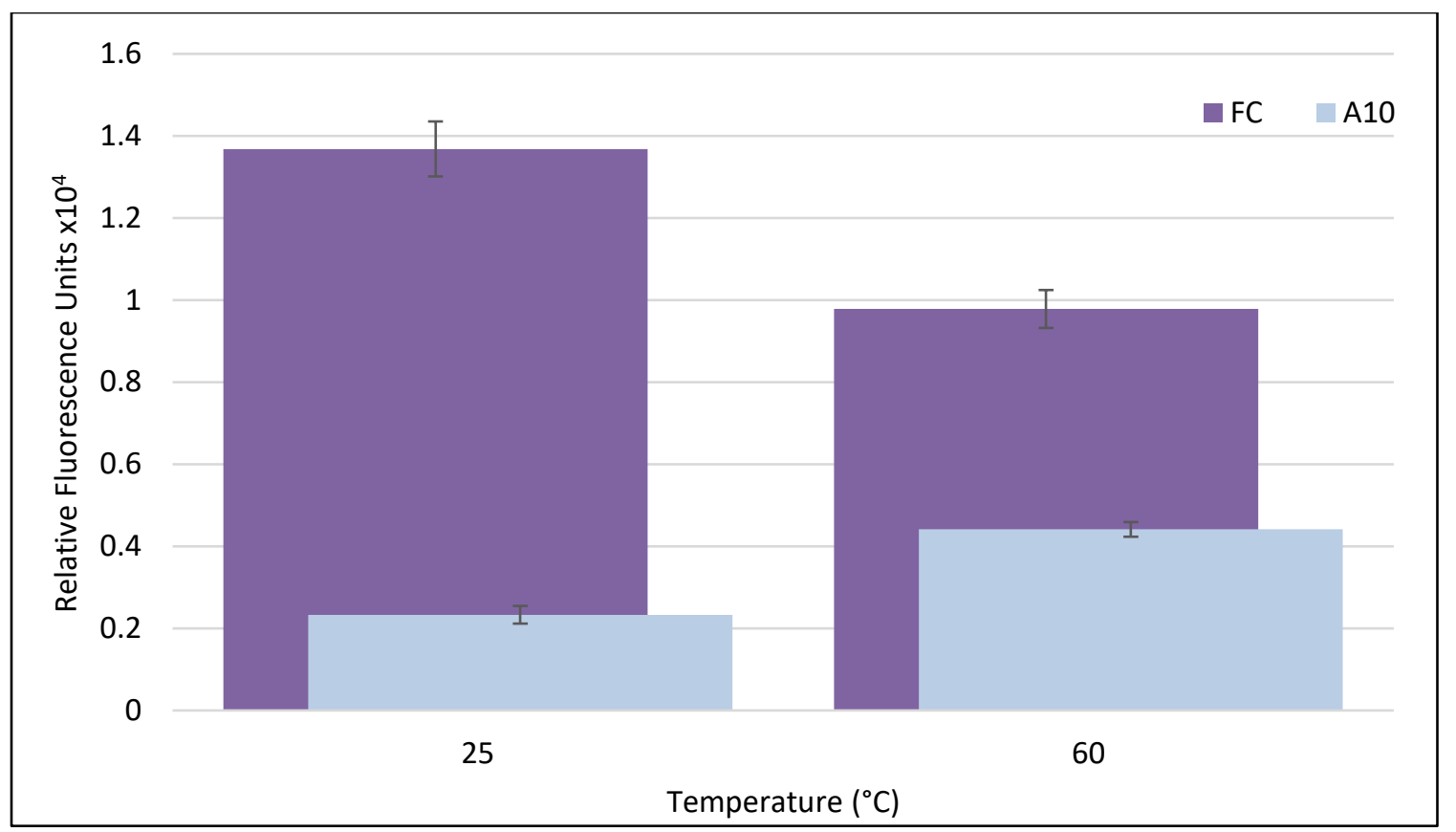

Figure A-5 - Fluorescence intensity of fluorescent control DNA (FC) and fluorescein / dabcyl labeled aptamer beacon with 10 complementary bases (A10) at 5.7 nM in TEB buffer. About 40 minutes passed while heating to $60^{\circ} \mathrm{C}$.

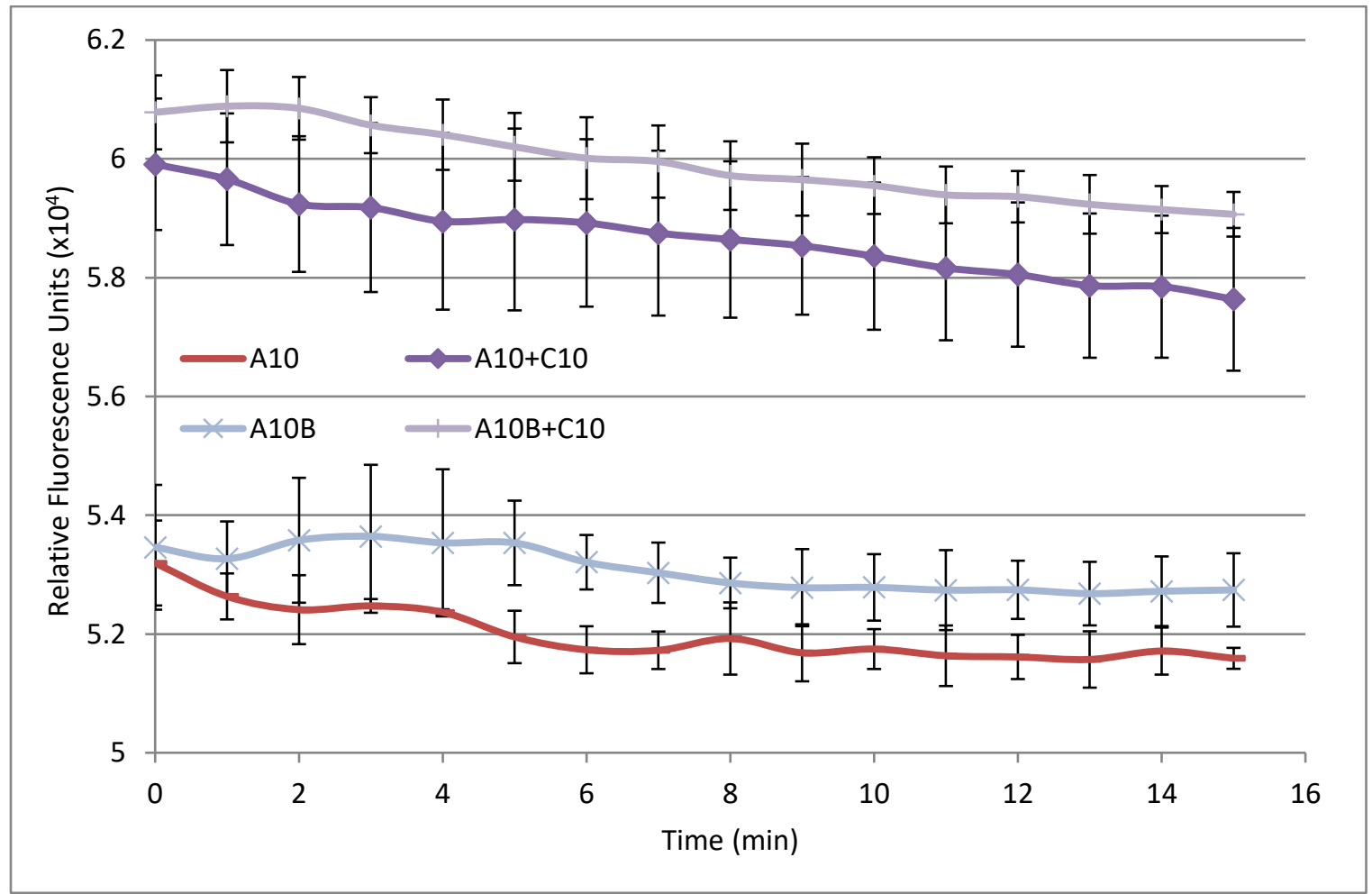

Figure A-6 - Fluorescence intensity effects of refolding in TEB buffer (B) of fluorescein / dabcyl labeled aptamer beacon with 10 complementary bases (A10) (100 nM) alone and in the presence of C10 (400 nM). Fluorescent control DNA (FC) had a value of about $27 \times 10^{4}$ RFUs. 


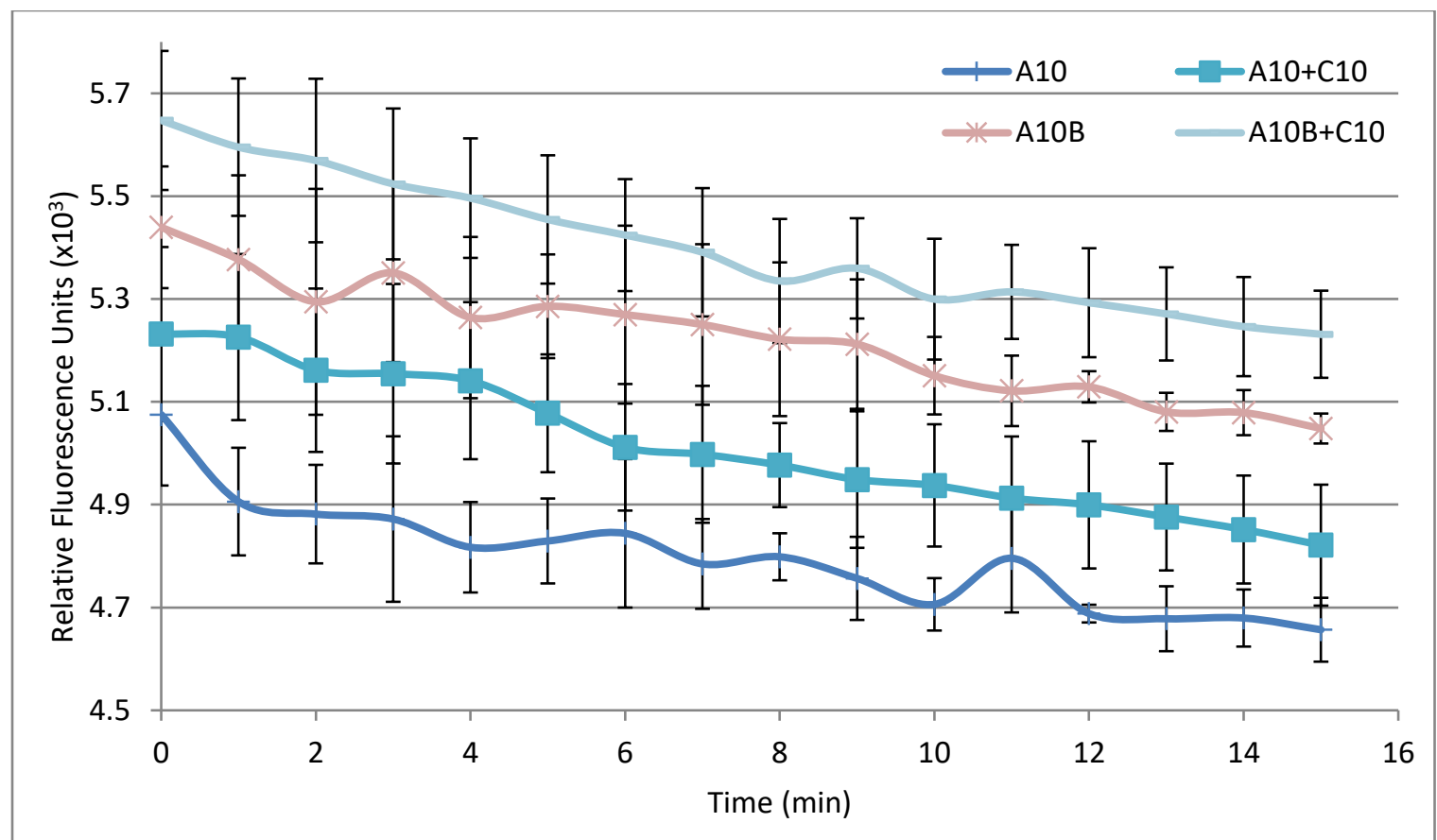

Figure A-7 - Fluorescence intensity effects of refolding in TEB buffer (B) of fluorescein / dabcyl labeled aptamer beacon with 10 complementary bases $(A 10)(10 \mathrm{nM})$ alone and in the presence of C10 (40 nM). Fluorescent control DNA (FC) had a value of about $21 \times 10^{3}$ RFUs.

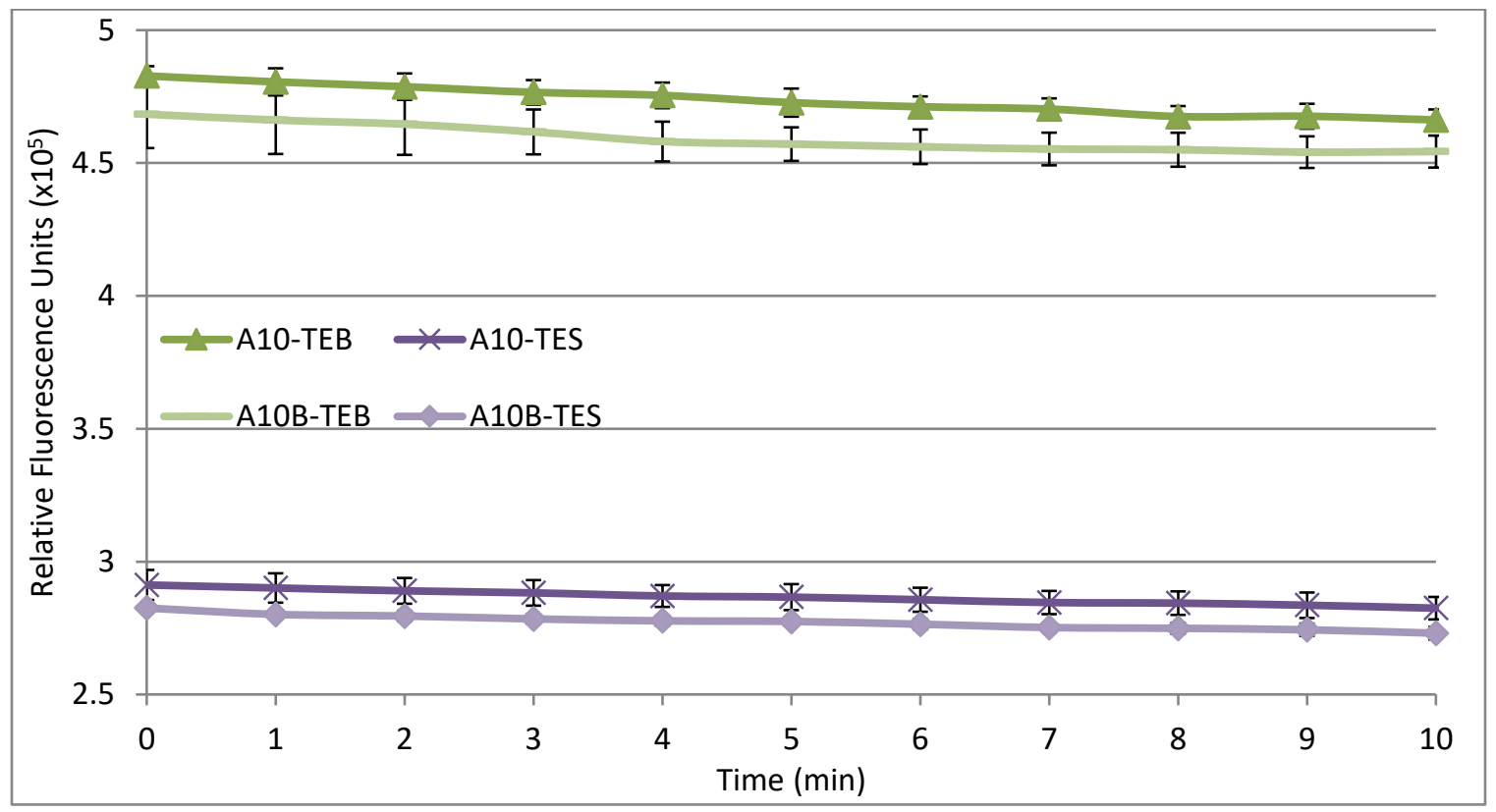

Figure A-8 - The effects of buffer salinity (TEB, 20mM; or TES, $1 \mathrm{M}$ ) and refolding in buffer (B) on background fluorescence of fluorescein / dabcyl labeled aptamer beacon with 10 complementary bases (A10) $(1 \mu \mathrm{M})$. Fluorescent control DNA (FC) values were around $35 \times 10^{5}$ RFUs. Ex. $495 \pm 10$ $\mathrm{nm}, \mathrm{Em} .528 \pm 20 \mathrm{~nm}$. 


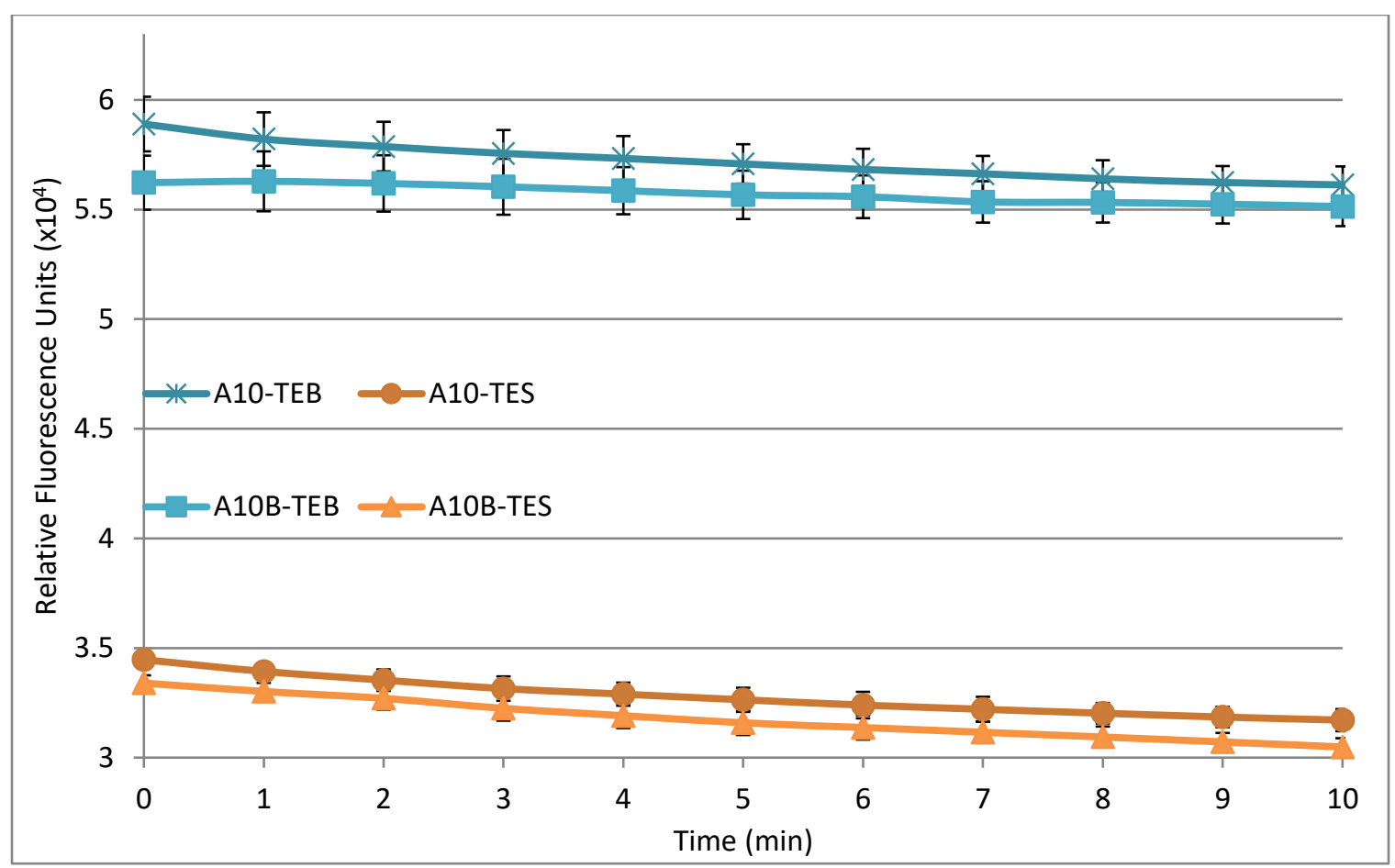

Figure A-9 - The effects of buffer salinity (TEB, 20mM; or TES, 1 M) and refolding in buffer (B) on background fluorescence of fluorescein / dabcyl labeled aptamer beacon with 10 complementary bases (A10) (100 nM). Fluorescent control DNA (FC) values were around $30 \times 10^{4}$ RFUs.

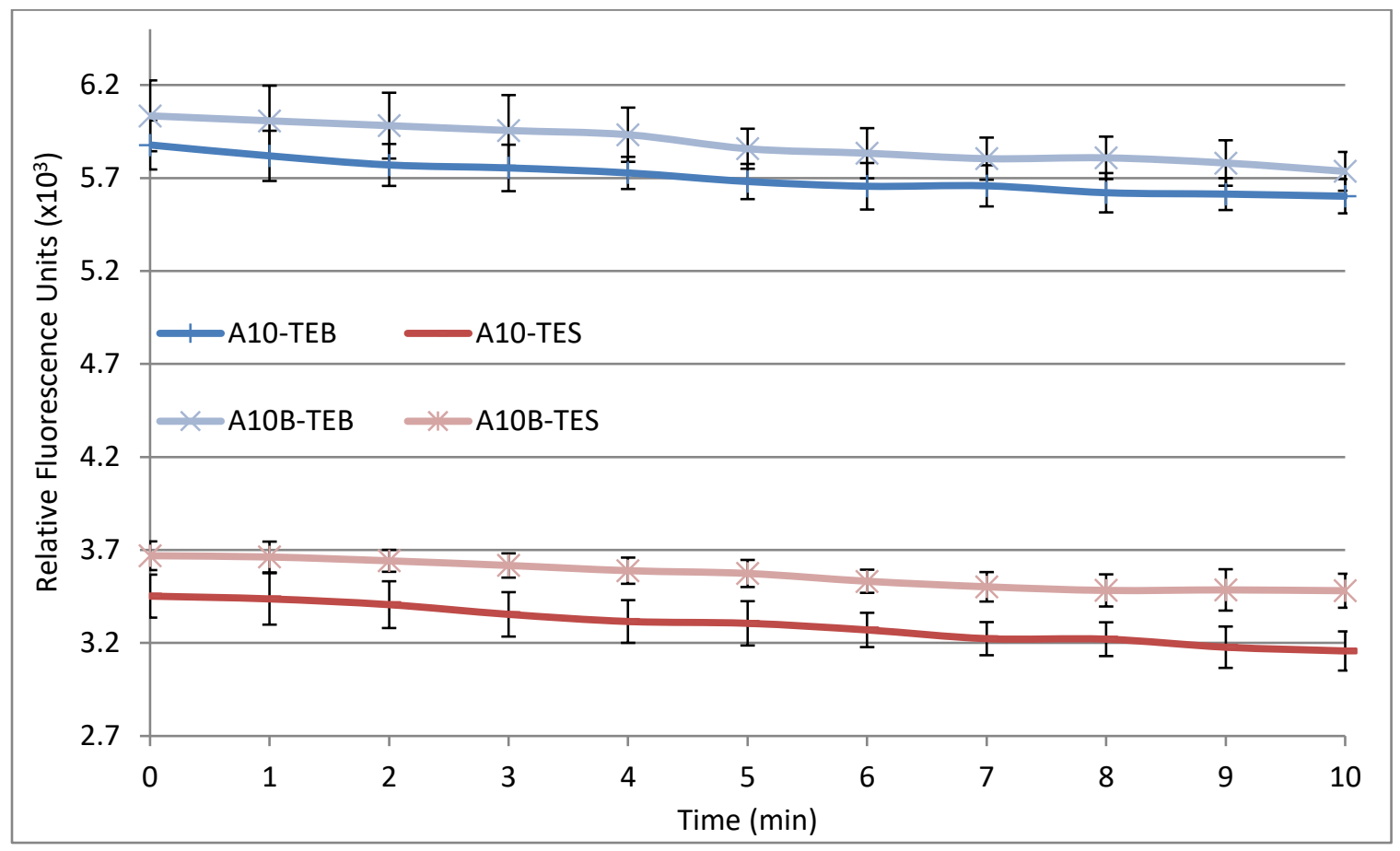

Figure A-10 - The effects of buffer salinity (TEB, 20mM; or TES, $1 \mathrm{M})$ and refolding in buffer (B) on background fluorescence of fluorescein / dabcyl labeled aptamer beacon with 10 complementary bases (A10) (10 nM). Fluorescent control DNA (FC) values were around $22 \times 10^{3}$ RFUs. 


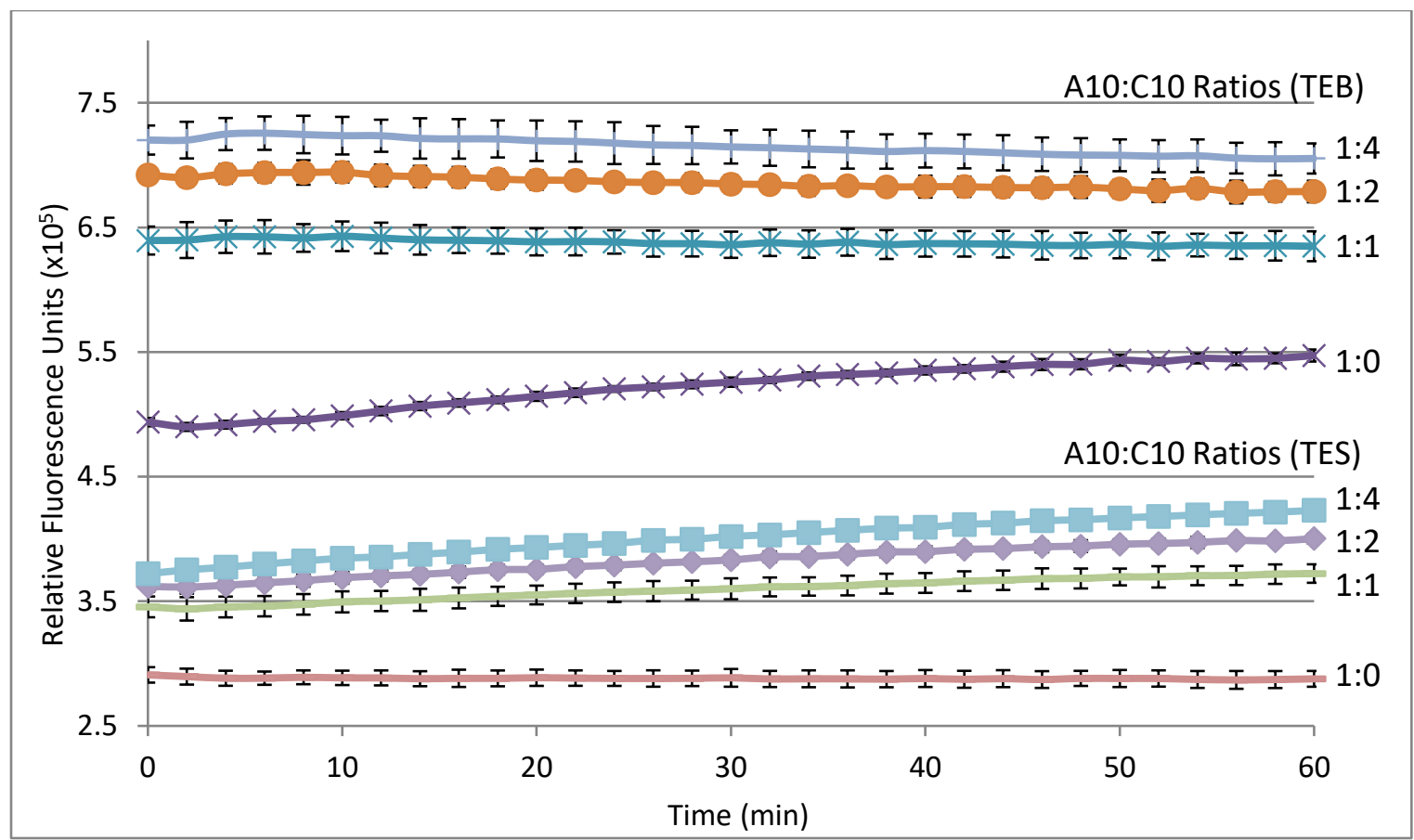

Figure A-11 - Fluorescence intensity of fluorescein / dabcyl labeled aptamer beacon with 10 complementary bases (A10) (1 $\mathrm{MM})$ in TEB or TES at various A10:C10 ratios after refolding in buffer. Fluorescent control DNA (FC) values were about $34 \times 10^{5}$ RFUs. Ex. $495 \pm 10 \mathrm{~nm}$, Em. 528 $\pm 20 \mathrm{~nm}$.

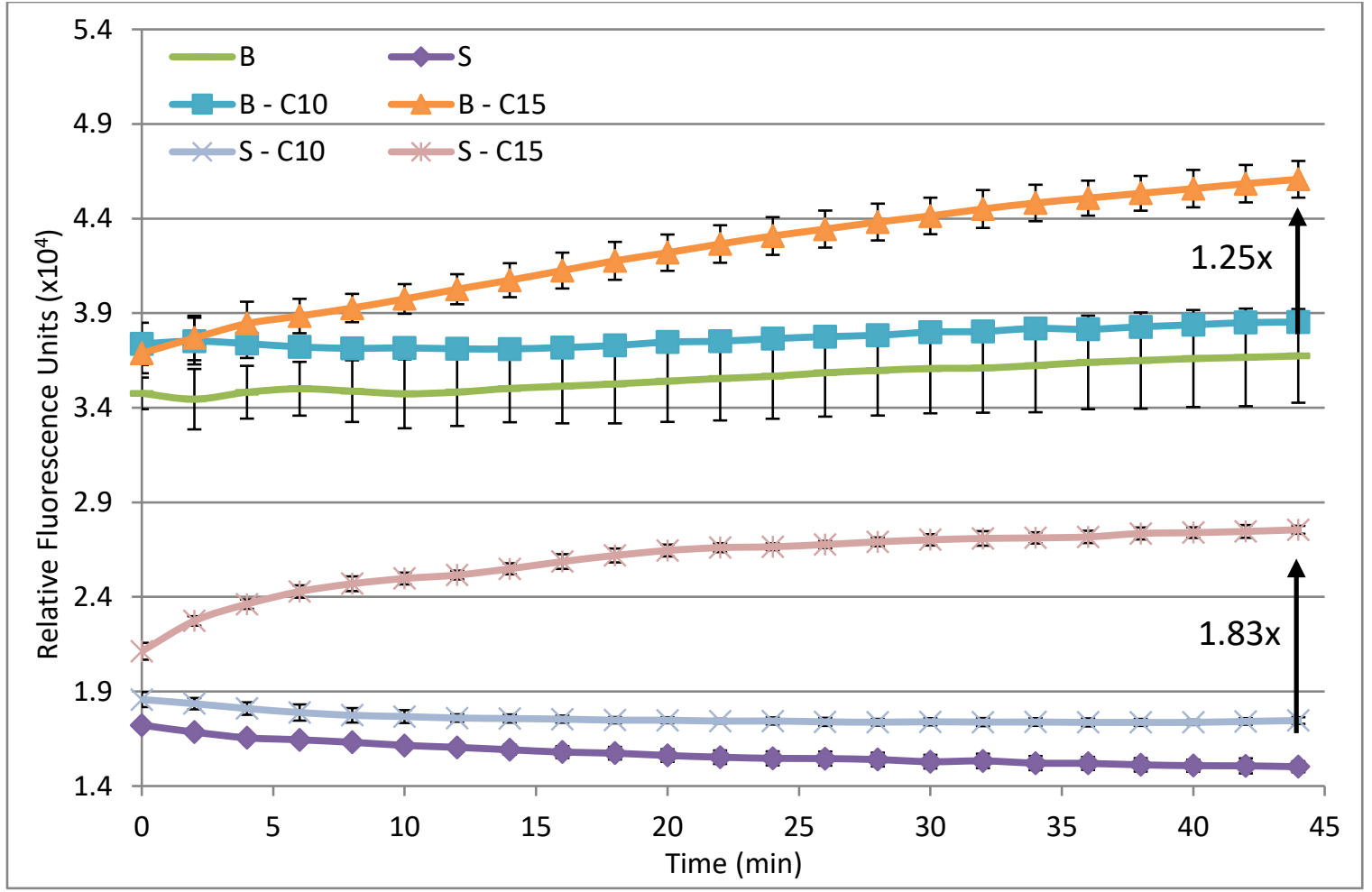

Figure A-12 - Fluorescence intensity of fluorescein / dabcyl labeled aptamer beacon with 10 complementary bases (A10) (100 nM) in the presence of C10 or C15 (100 nM) in TEB (B) and TES (S). Fluorescent control DNA (FC) values were about $25 \times 10^{4}$ RFUs. 


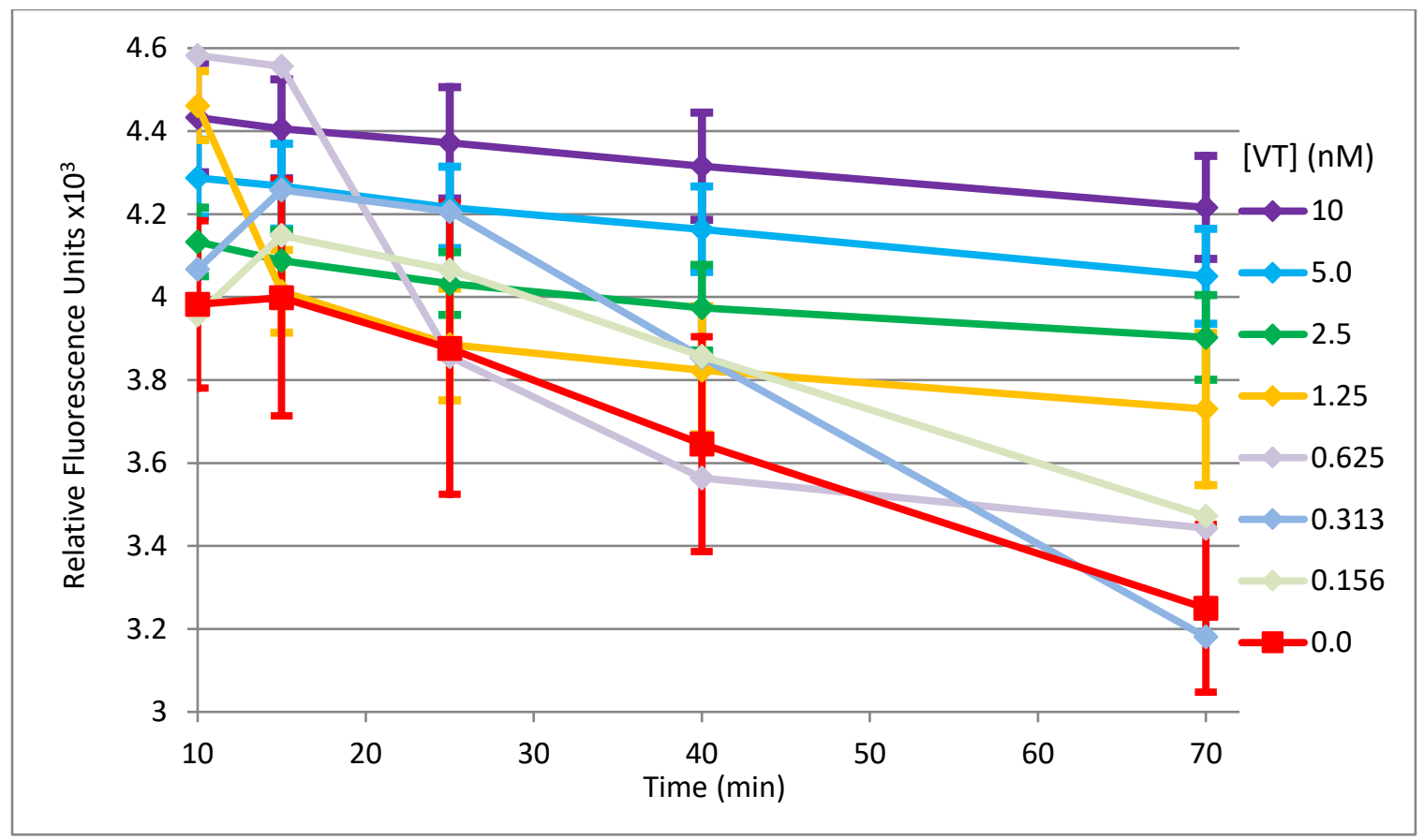

Figure A-13 - Fluorescence of fluorescein / dabcyl labeled aptamer beacon (FLQ) (10 nM) measured over time with varying VT1 concentrations in TES buffer. Fluorescein labeled aptamer (FLX) values (10 nM) were around $17 \times 10^{3}$ RFUs. Data points represent the average of 6 wells from replicates 1 and 2. Error bars shown are SD. Ex. $495 \pm 10 \mathrm{~nm}, \mathrm{Em} .528 \pm 20 \mathrm{~nm}$.

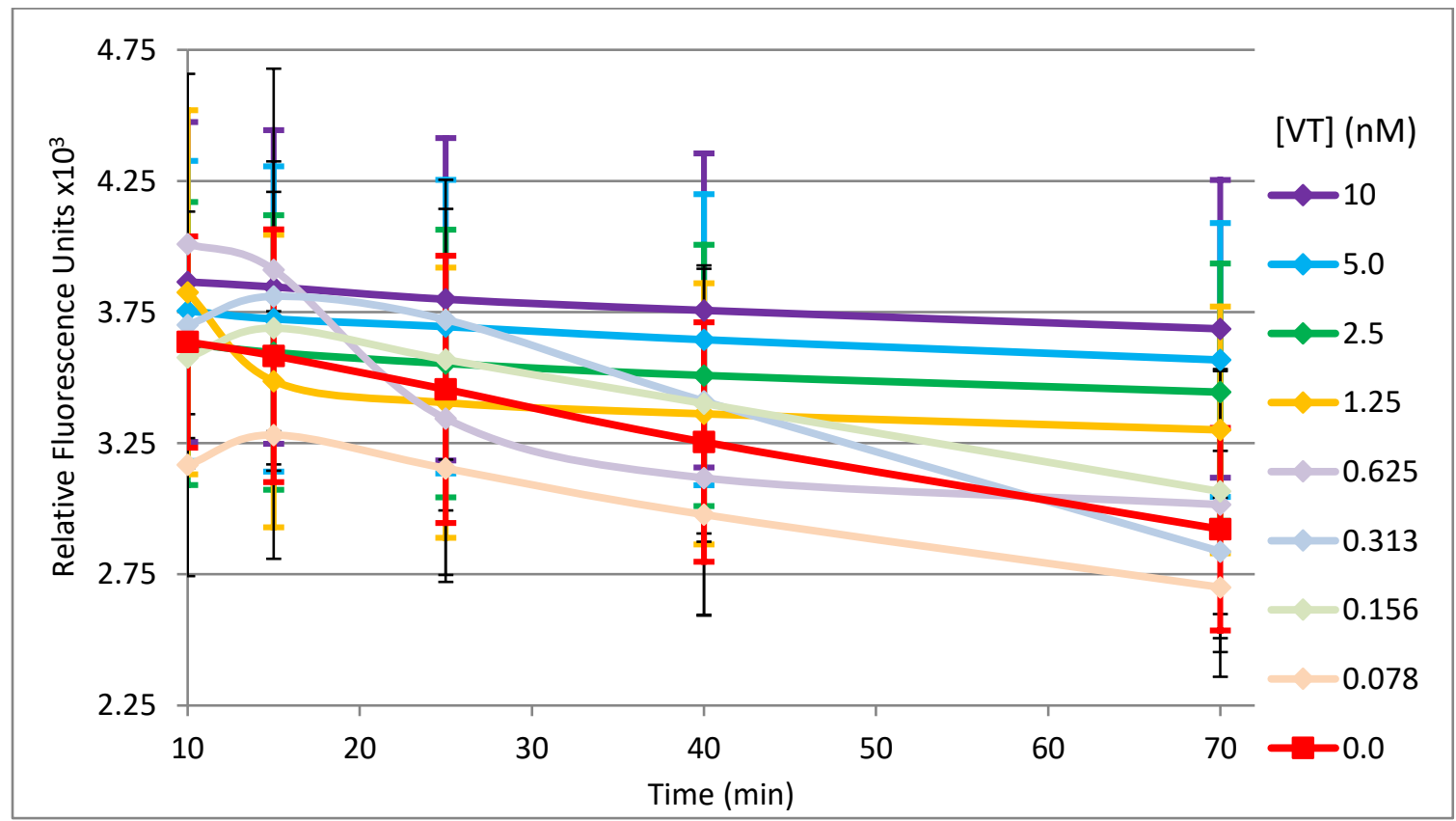

Figure A-14 - Fluorescence of fluorescein / dabcyl labeled aptamer beacon (FLQ) (10 nM) measured over time with varying VT1 concentrations in TES buffer. Fluorescein labeled aptamer (FLX) values $(10 \mathrm{nM})$ were around $17 \times 10^{3}$ RFUs. Data points represent the average of 12 wells over 4 replicates. Error bars represent SD. Ex. $495 \pm 10$ nm, Em. $528 \pm 20 \mathrm{~nm}$. 


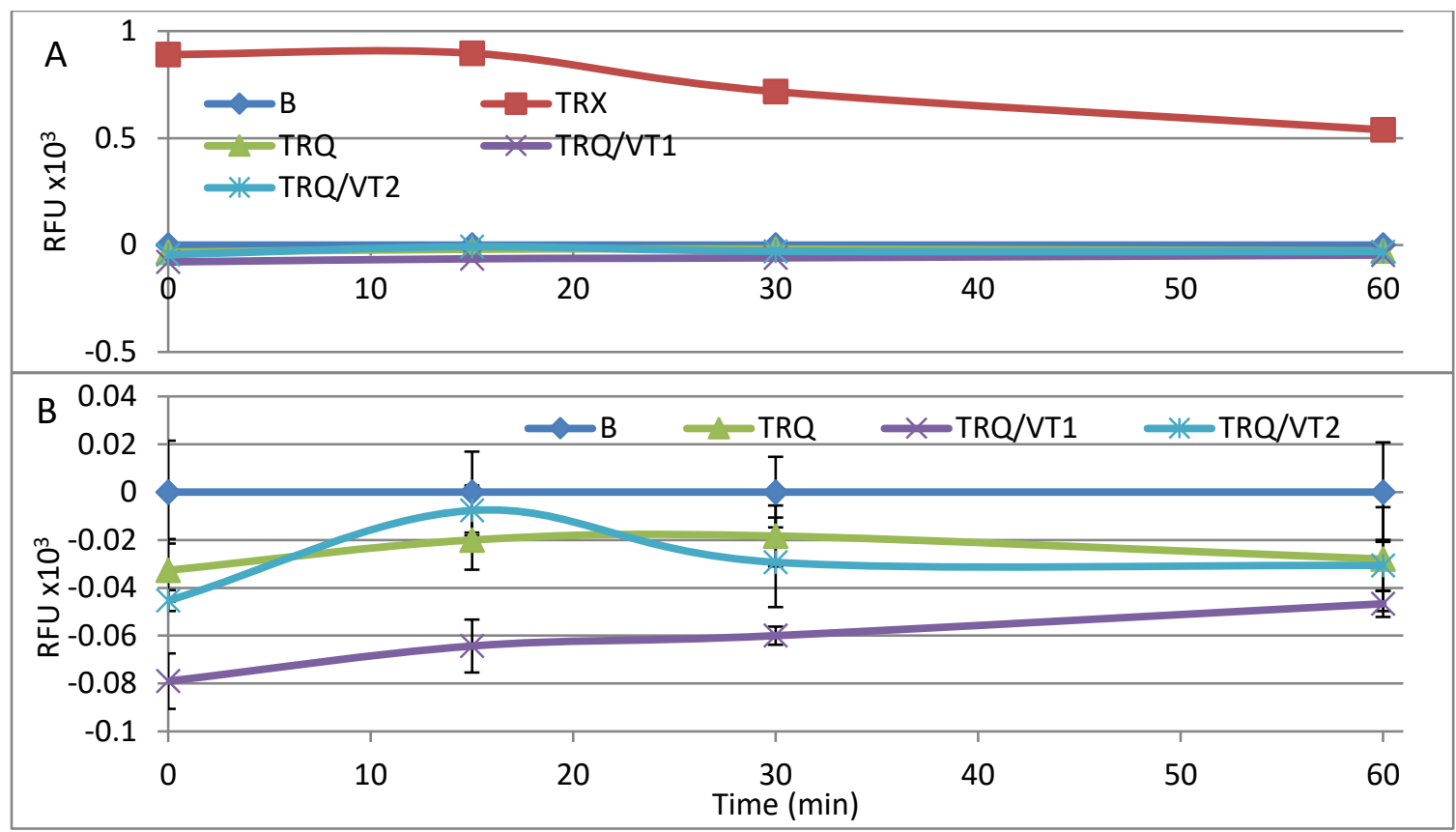

Figure A-15 - Blanked change in fluorescence over time for the Texas Red / Black Hole Quencher 2 labeled aptamer beacon (TRQ) (10 nM) in the presence of VT1a or VT2a (each at $10 \mathrm{nM}$ ). A) Full view including unquenched Texas Red labeled aptamer (TRX) sequence; $B$ ) close-up on curves excluding Cyanine 5 labeled aptamer (CYX). Data points are the average of triplicate readings. Error bars show SD. Ex. $596 \pm 15 \mathrm{~nm}, \mathrm{Em} .680 \pm 30 \mathrm{~nm}$

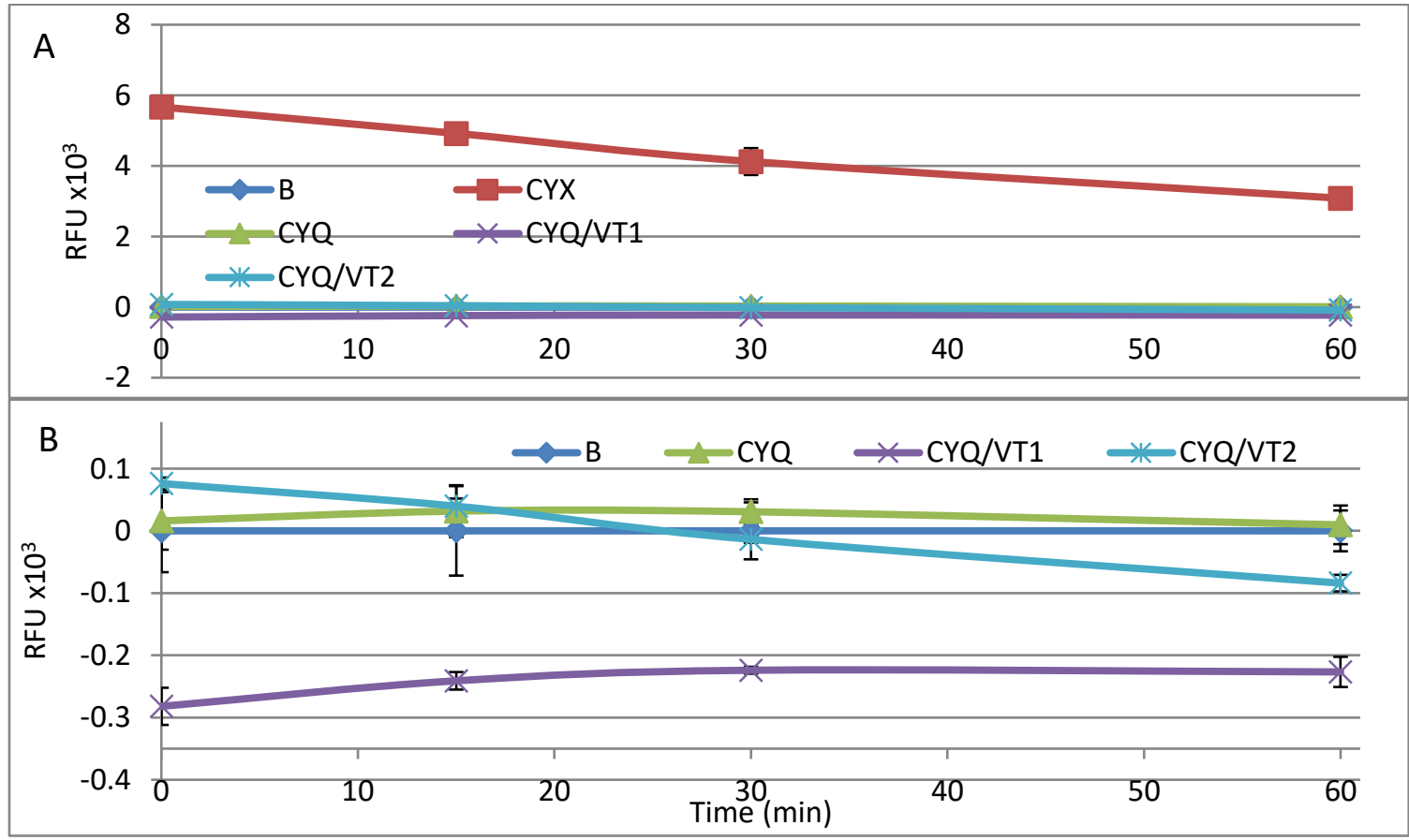

Figure A-16 - Blanked change in fluorescence over time for Cyanine 5 / Black Hole Quencher 3 labeled aptamer beacon (CYQ) (10 nM) in the presence of VT1a or VT2a (each at 10 nM). A) Full view including unquenched Cyanine 5 labeled aptamer (CYX) sequence; B) close-up on curves excluding CYX. Data points are the average of triplicate readings. Error bars show SD. Ex. $620 \pm$ $40 \mathrm{~nm}, \mathrm{Em} .680 \pm 30 \mathrm{~nm}$. 


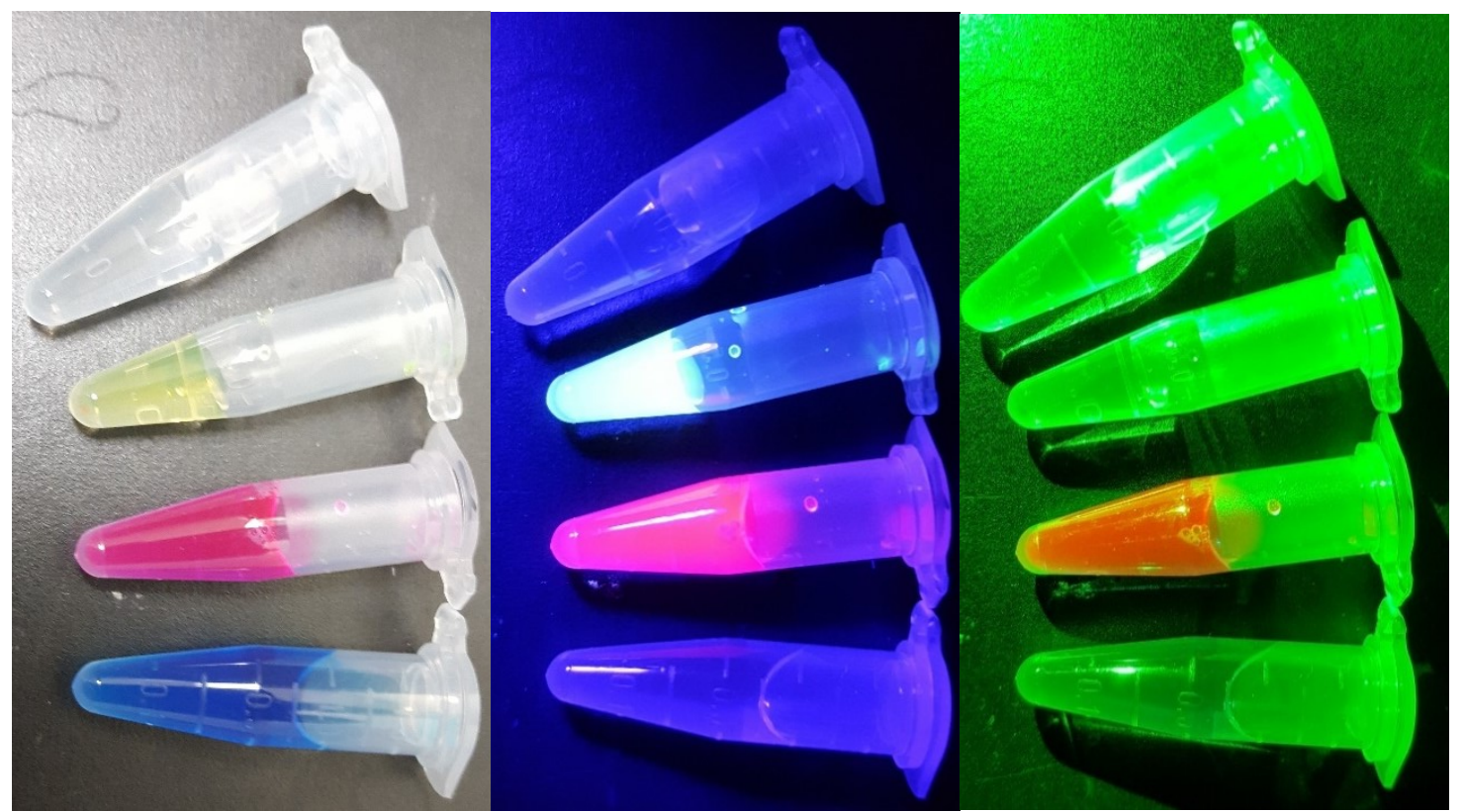

Figure A-17 - Comparison of water and $100 \mu$ M solutions of fluorescein, Texas Red, and NIR-641 (top to bottom) illuminated by white light, handheld UV lamp, and green LED flashlight (left to right). Images taken by cellphone camera.

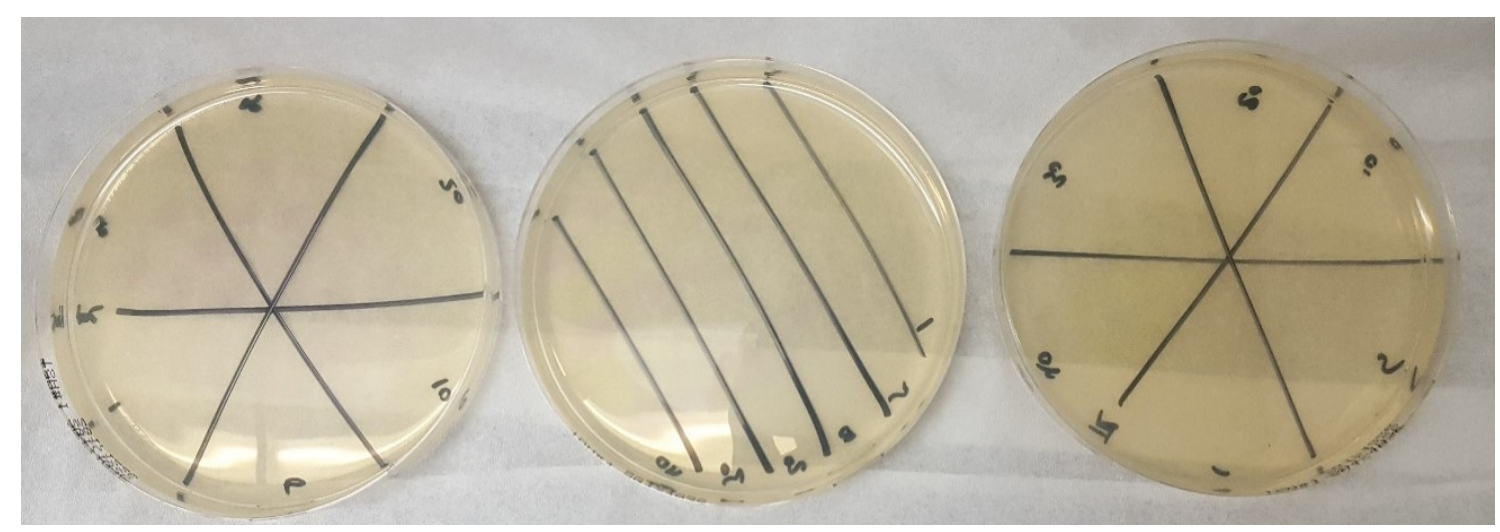

Figure A-18 - TSA plates used for visible fluorescence comparison in white light. Texas Red (left), both (middle), fluorescein (right). Image taken with a cell phone camera. 


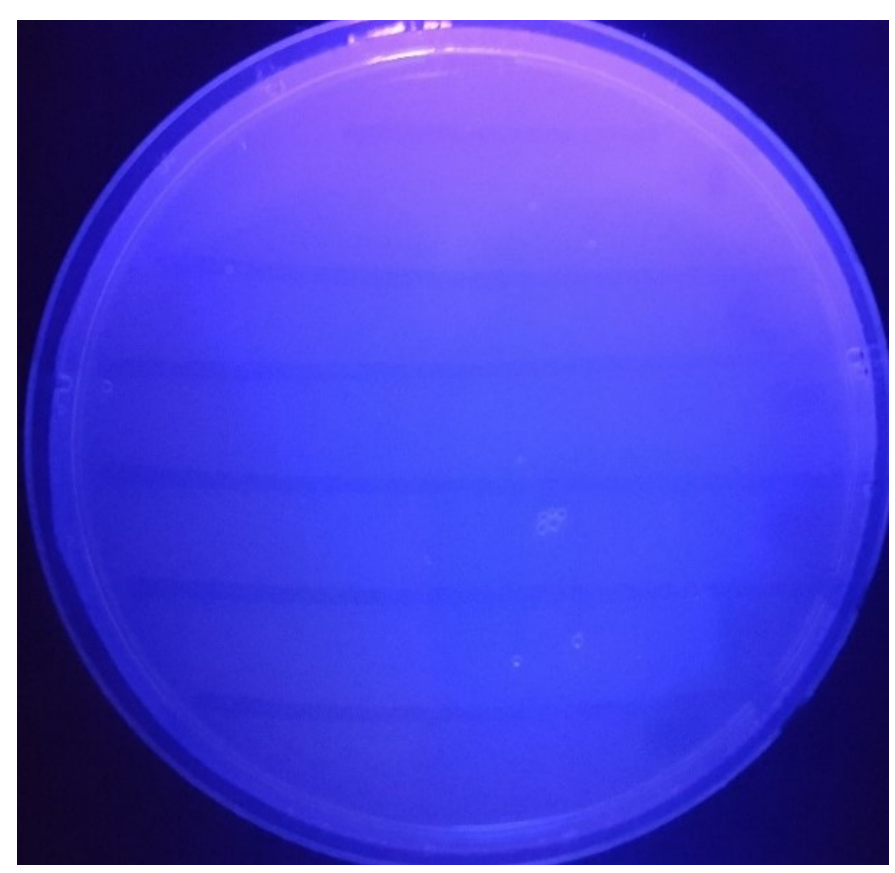

Figure A-19 - Comparison of visible fluorescence of fluorescein and Texas Red spotted on a 1.5\% agarose plate. Solutions were between $40-1 \mu \mathrm{M}$. Samples were spotted in triplicate and the plate was refrigerated overnight to allow spots to dry. Image taken with a cell phone camera.

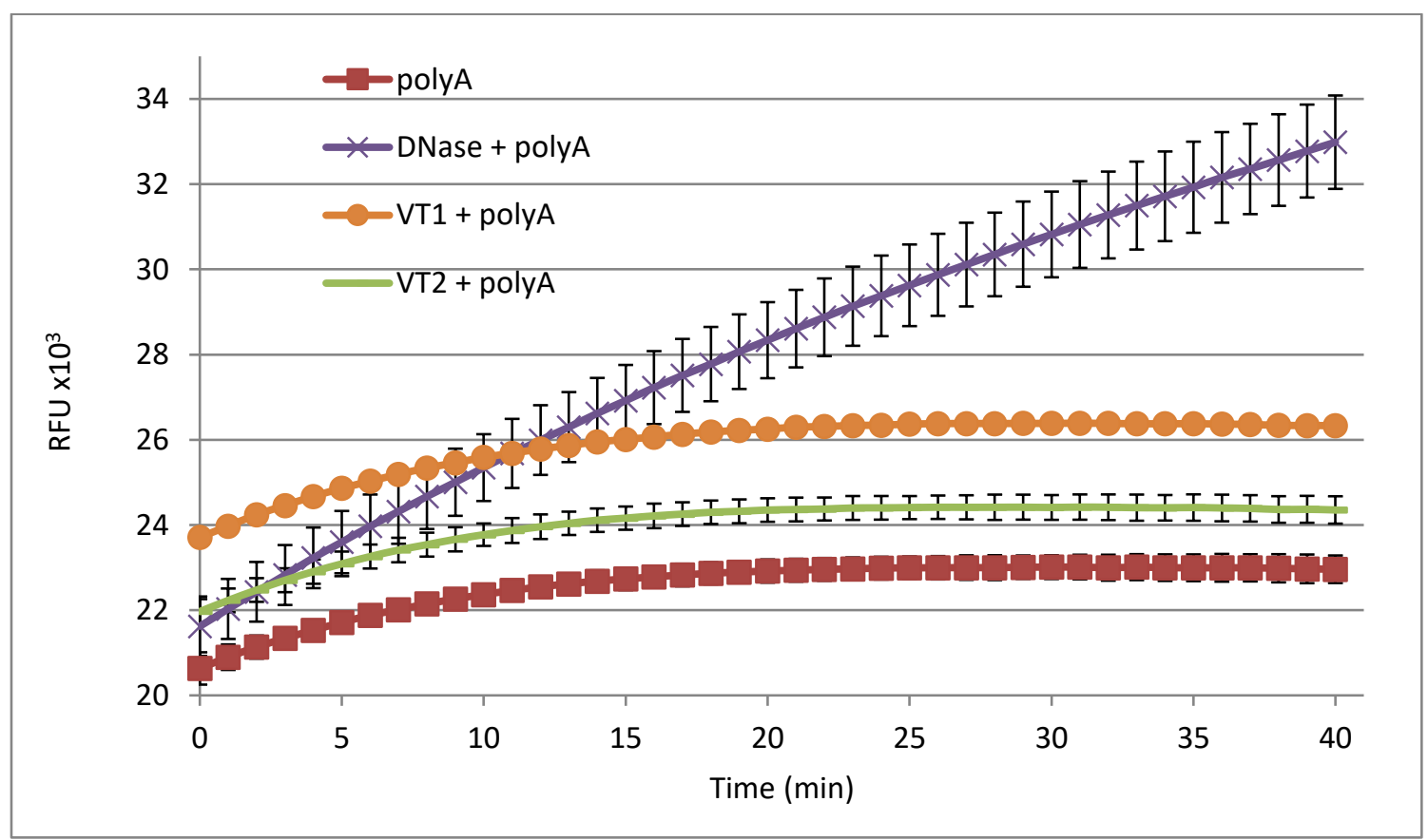

Figure A-20 - Fluorescence values for the first enzyme activity assay for the first 40-minute incubation at $30^{\circ} \mathrm{C}$. Solutions contained DNase (0.5 units per $\left.100 \mu \mathrm{L}\right)$, VT1 or VT2 (10 nM), in addition to polyA (3.75 $\mu \mathrm{M})$ in EAB. Data points are the average of triplicate. Error bars are SD. 


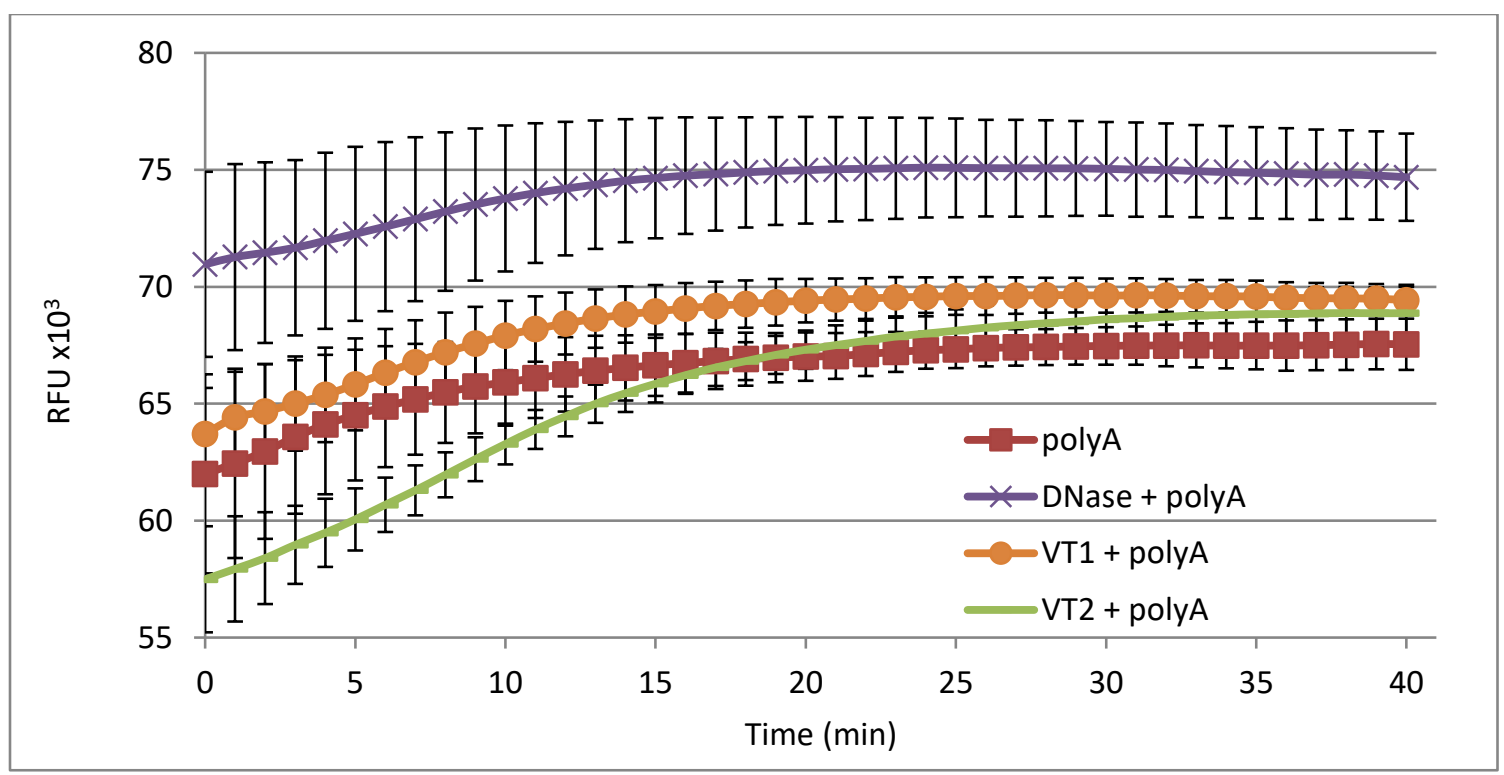

Figure A-21 - Fluorescence values for the first enzyme activity assay for the second 40-minute incubation at $30^{\circ} \mathrm{C}$. Solutions contained DNase (0.5 units per $100 \mu \mathrm{L}$ ), VT1 or VT2 (10 nM), crude $V T 2 a(117 \mathrm{nM})$, in addition to polyA (3.75 $\mu \mathrm{M})$ and $\mathrm{N}, \mathrm{N}^{\prime}$-dimethyl-ethylene-diamine $(100 \mathrm{mM})$ in $E A B$. Data points are the average of triplicate. Error bars are $S D$

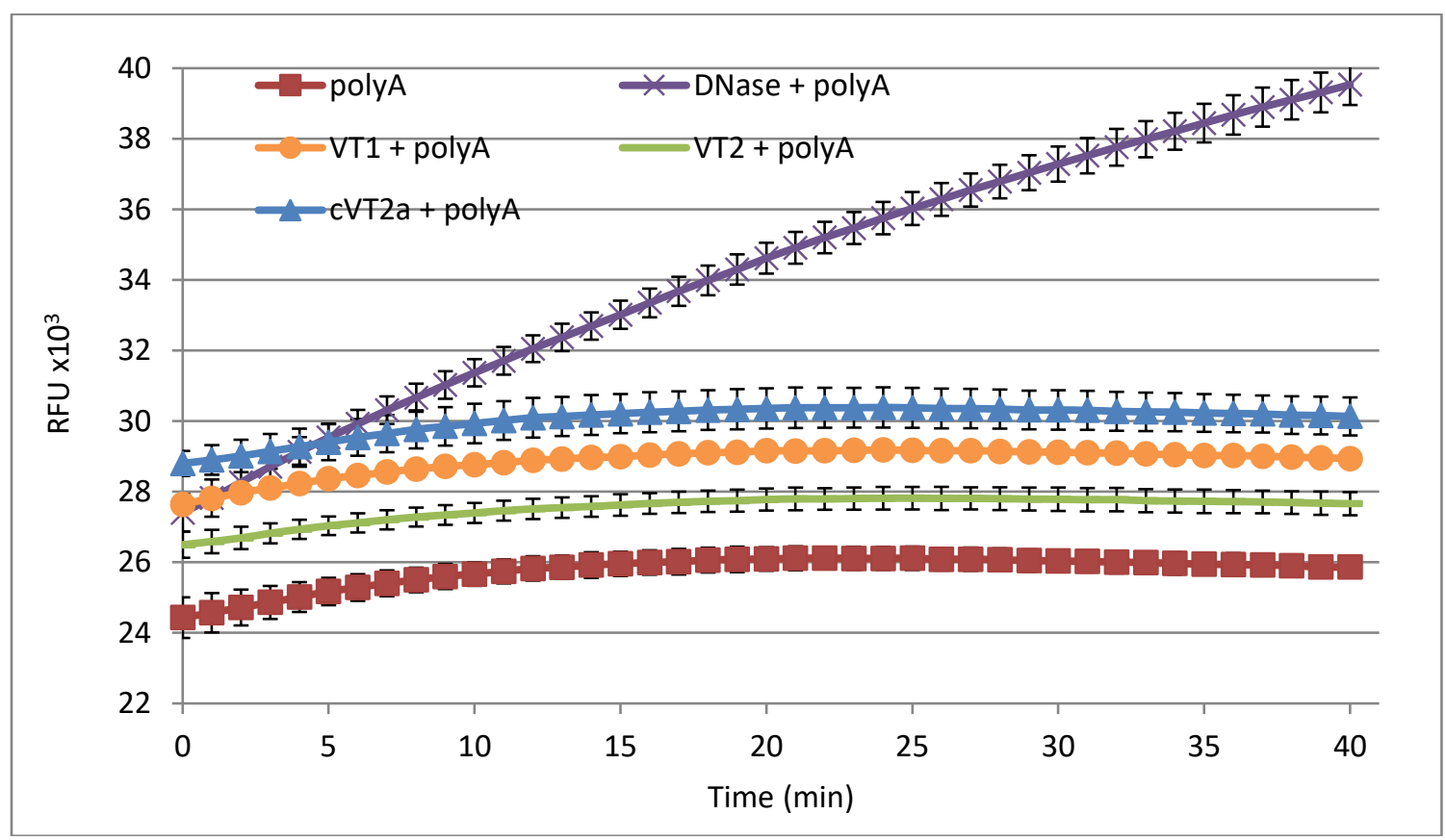

Figure A-22 - Fluorescence values for the second enzyme activity assay for the first 40-minute incubation at $30^{\circ} \mathrm{C}$. Solutions contained DNase (0.5 units per $\left.100 \mu \mathrm{L}\right)$, VT1 or VT2 (10 nM), crude $V T 2 a(117 \mathrm{nM})$, in addition to polyA (3.75 $\mu \mathrm{M})$ in EAB. Data points are the average of triplicate. Error bars are $S D$ 


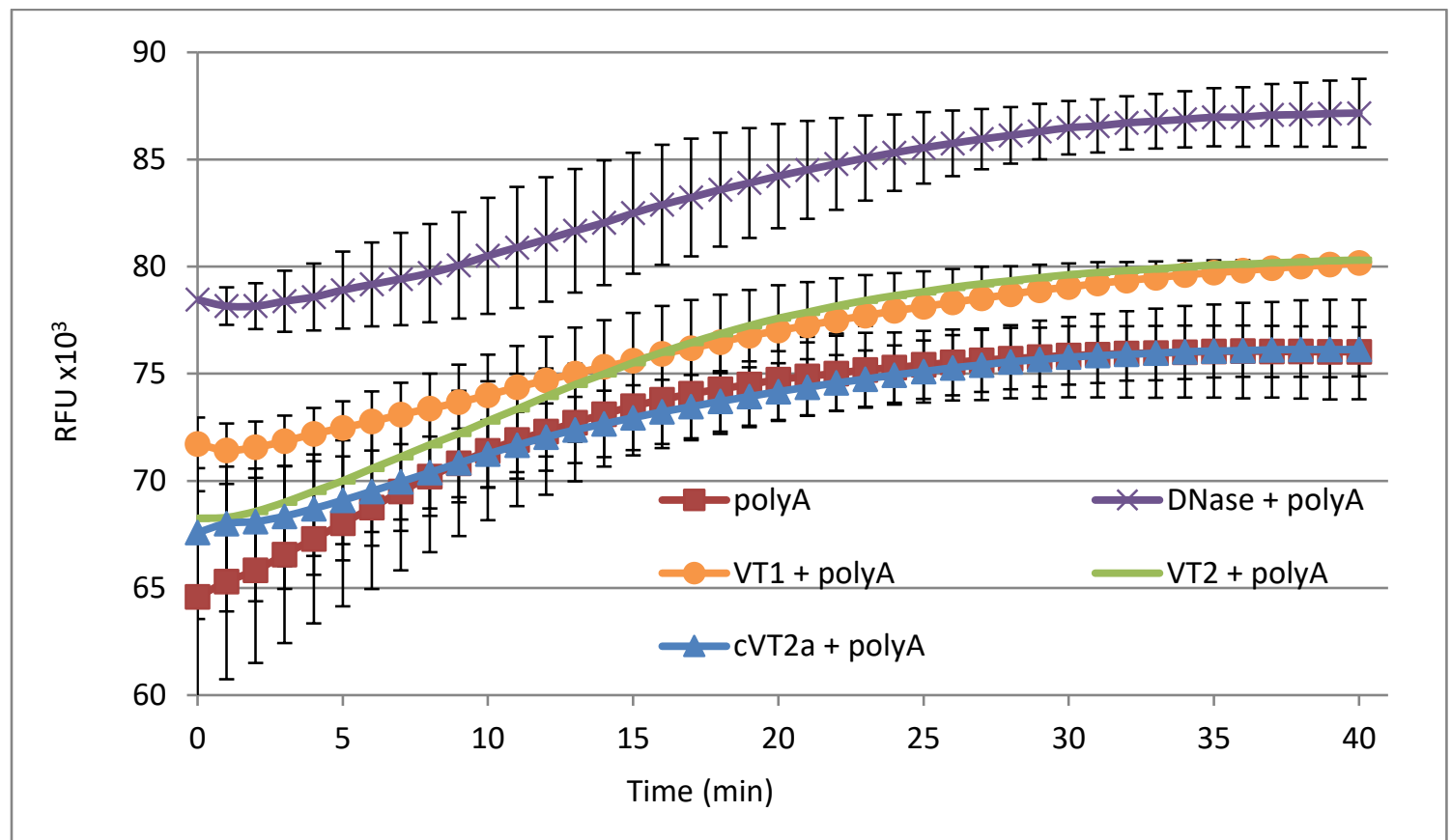

Figure A-23 - Fluorescence values for the second enzyme activity assay for the second 40-minute incubation at $30^{\circ} \mathrm{C}$. Solutions contained DNase (0.5 units per $\left.100 \mu \mathrm{L}\right)$, VT1 or VT2 (10 nM), crude VT2a (117 $\mathrm{nM})$, in addition to polyA (3.75 $\mu \mathrm{M})$ and $N, N^{\prime}$-dimethyl-ethylene-diamine $(100 \mathrm{mM})$ in $E A B$. Data points are the average of triplicate. Error bars are $S D$.

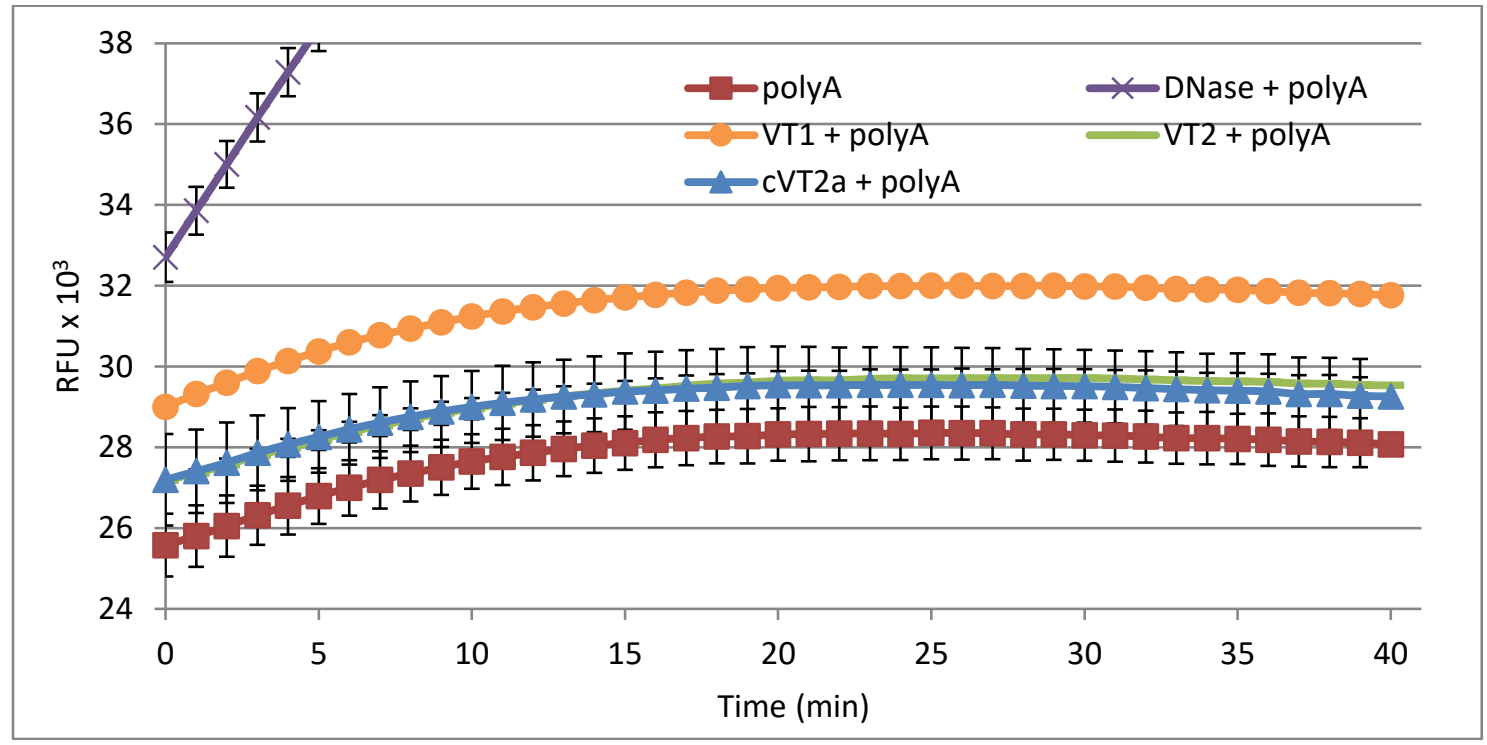

Figure A-24 - Fluorescence values for the third enzyme activity assay for the first 40-minute incubation at $30^{\circ} \mathrm{C}$. Solutions contained DNase (0.5 units per $\left.100 \mu \mathrm{L}\right)$, VT1 or VT2 or crude VT2a $(10 \mathrm{nM})$, in addition to polyA $(3.75 \mu \mathrm{M})$ in EAB. Data points are the average of duplicate. Error bars are $S D$ 


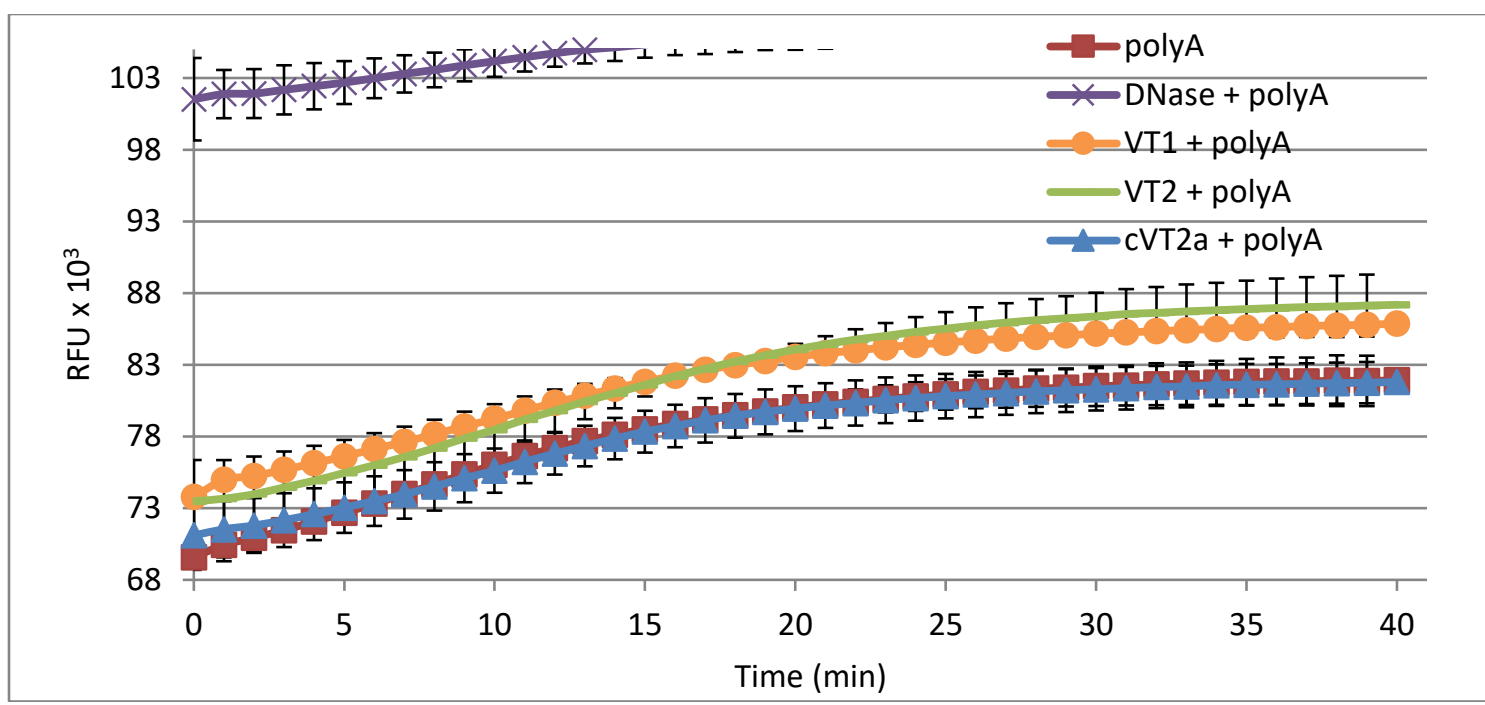

Figure A-25 - Fluorescence values for the third enzyme activity assay for the second 40-minute incubation at $30^{\circ} \mathrm{C}$. Solutions contained DNase (0.5 units per $\left.100 \mu \mathrm{L}\right)$, VT1 or VT2 or crude VT2a $(10 \mathrm{nM})$, in addition to polyA (3.75 $\mu \mathrm{M})$ and $N, N^{\prime}$-dimethyl-ethylene-diamine (100 mM) in EAB. Data points are the average of duplicate. Error bars are SD 


\section{Appendix B Additional Tables}

\section{List of Tables:}

Table A-1 - P-values for the significance between fluorescein / dabcyl labeled aptamer beacon (FLQ) and FLQ+VT1 seen in Figure 27. P-values based on a 1 tailed, paired student's t-test, $\alpha=0.05$. 
Table A-1 - P-values for the significance between fluorescein / dabcyl labeled aptamer beacon (FLQ) and FLQ+VT1 seen in Figure 27. P-values based on a 1 tailed, paired student's t-test, $\alpha=0.05$.

\begin{tabular}{|c|c|c|c|}
\hline Concentration (nM) & 25 & 40 & 70 \\
\hline 10 & 0.0248 & 0.0032 & 0.0003 \\
\hline 5 & 0.0545 & 0.0072 & 0.0007 \\
\hline 2.5 & 0.194 & 0.0300 & 0.0012 \\
\hline 1.25 & 0.481 & 0.1664 & 0.0135 \\
\hline 0.625 & 0.470 & 0.3678 & 0.2079 \\
\hline 0.313 & $0.0313^{\#}$ & 0.1137 & 0.2825 \\
\hline 0.156 & 0.145 & 0.1091 & 0.1007 \\
\hline
\end{tabular}

\# Values higher 


\section{Appendix C Mass Spectrometry Reports}

The mass spectrometry display reports in the next 6 pages correspond to testing synthesized DNA oligonucleotides. The samples were given numbers as described here:

$2 B-B a n d B$ from $C 10$ synthesis gels

$4 B$ - Band B from fluorescein / dabcyl labeled aptamer beacon with 10 complementary bases (A10) synthesis gels

$6 \mathrm{~B}$ - Band B from fluorescein / dabcyl labeled aptamer beacon with 10 complementary bases (A15) synthesis gels

CCFL \#1 - Band B from complete complement DNA (CC) sequence synthesis gels

FL3 \#2 - Band B from Fluorescein labeled aptamer (FLX) synthesis gels

FLQ5 \#4 - Band B from fluorescein / dabcyl labeled aptamer beacon (FLQ) synthesis gels 
Mass spectrometric results for $2 \mathrm{~B}-\mathrm{C} 10$ sequences

\section{Display Report}

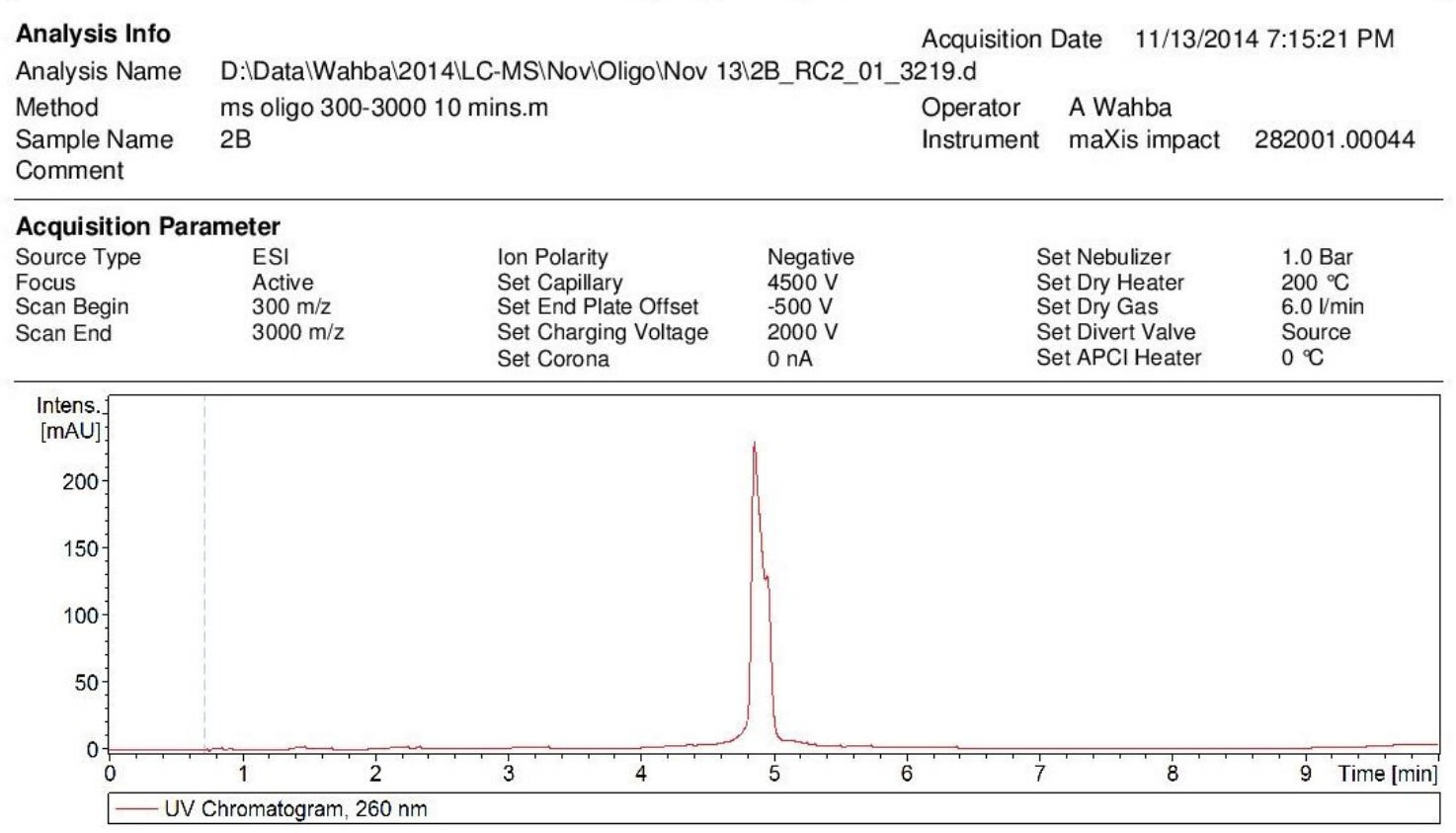
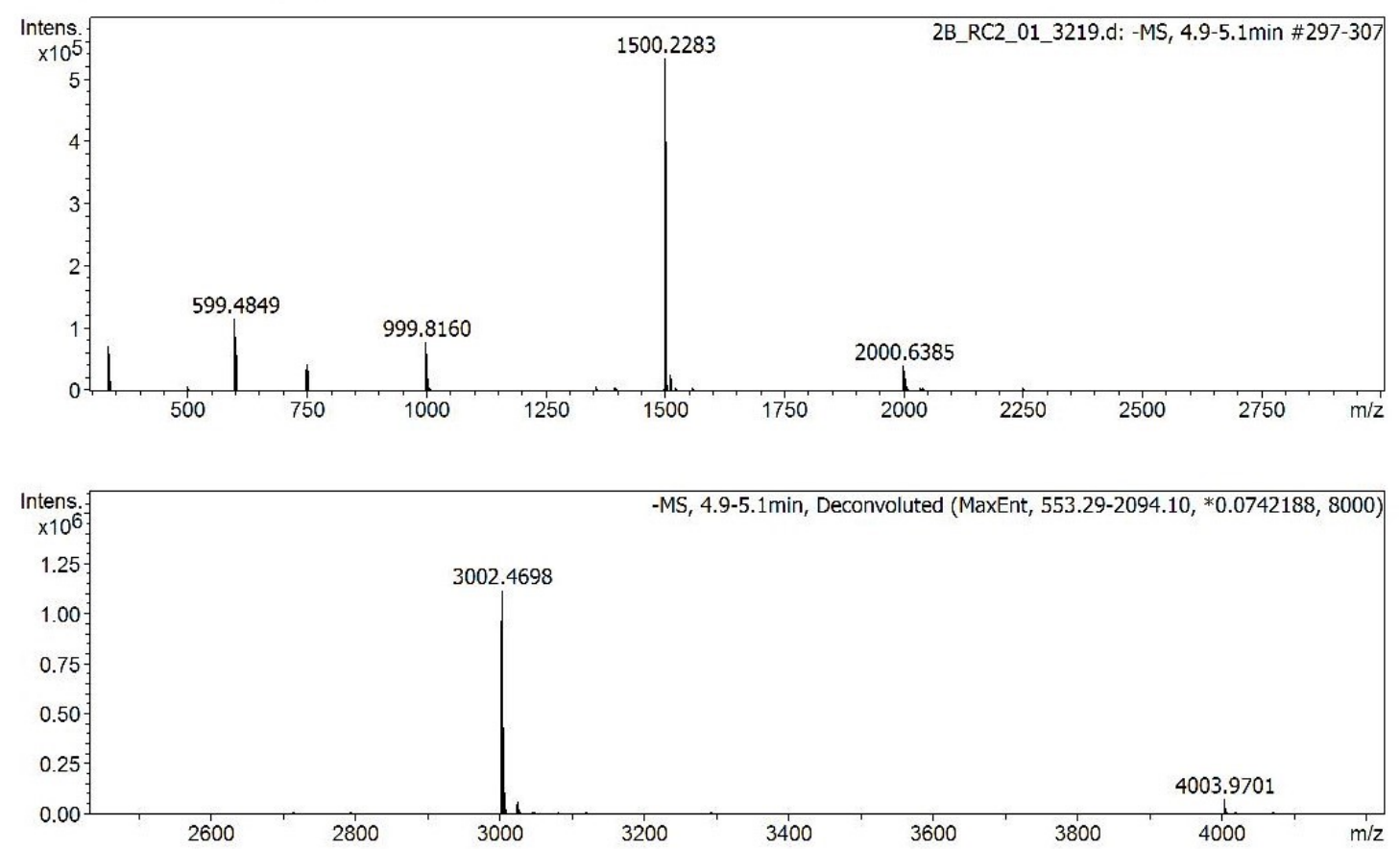

2B_RC2_01_3219.d 
Mass spectrometric results for 4B - Fluorescein / dabcyl labeled aptamer beacon with 10 complementary bases (A10) sequences
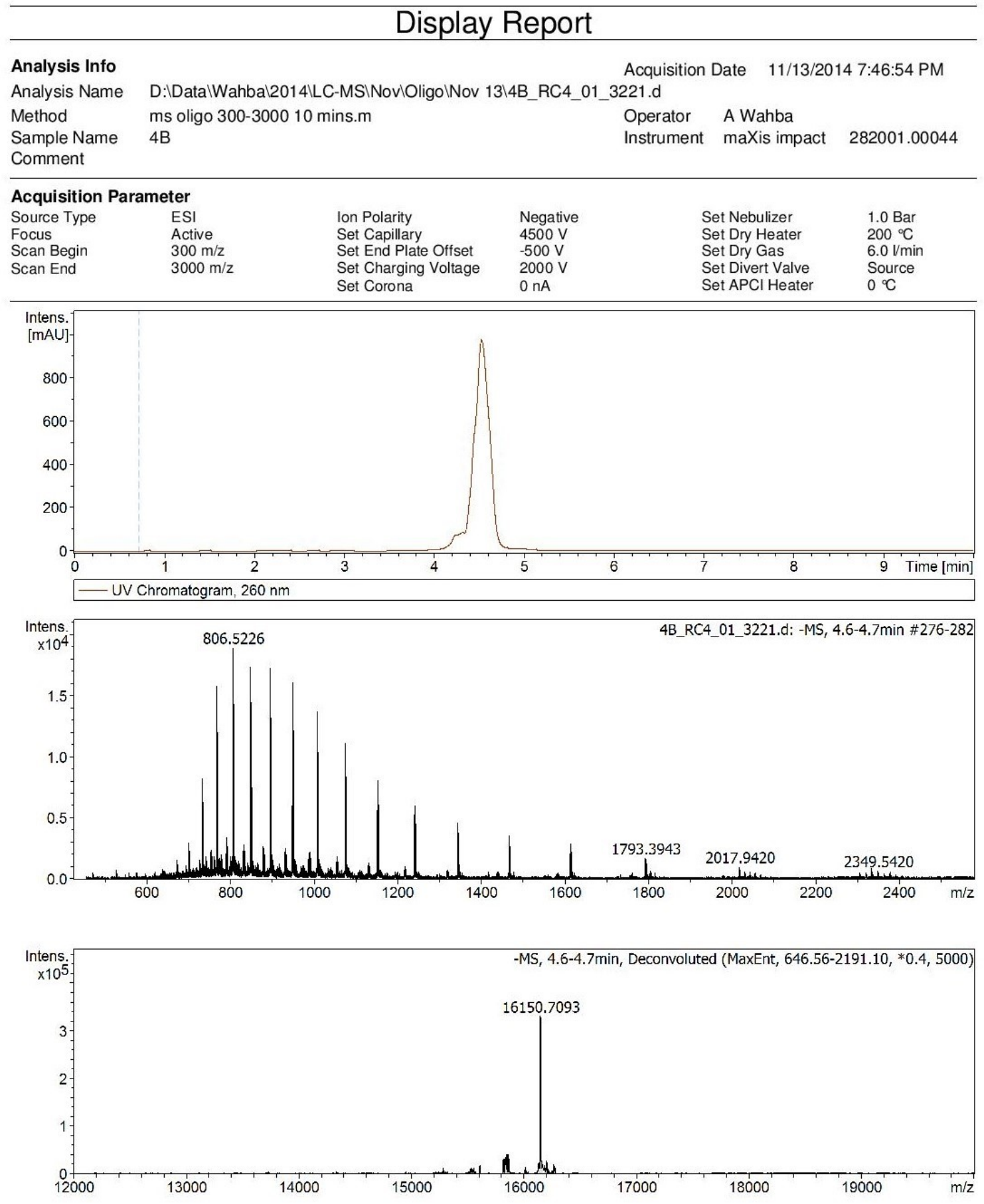

4B_RC4_01_3221.d 
Mass spectrometric results for 6B - Fluorescein / dabcyl labeled aptamer beacon with 10 complementary bases (A15) sequences

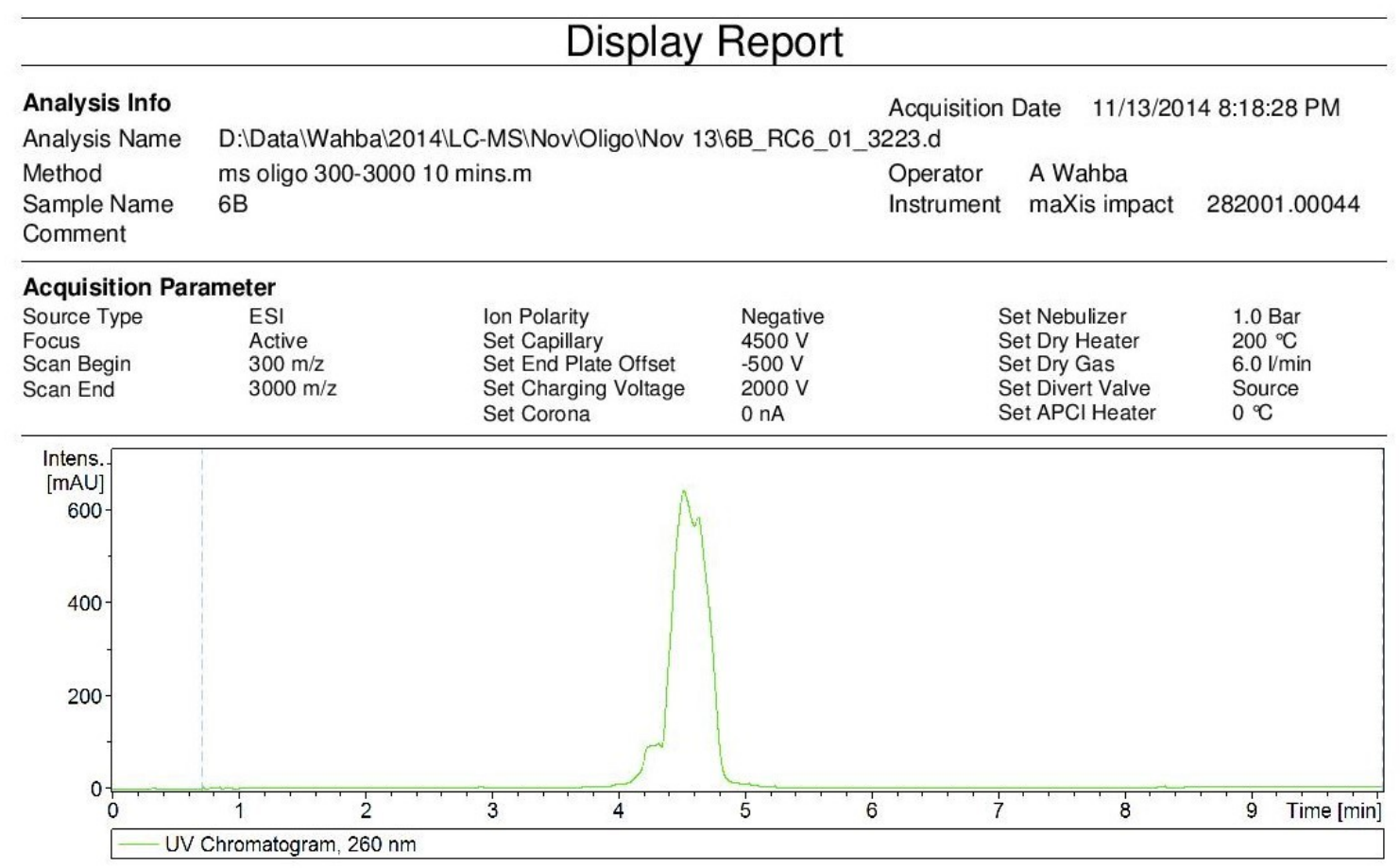
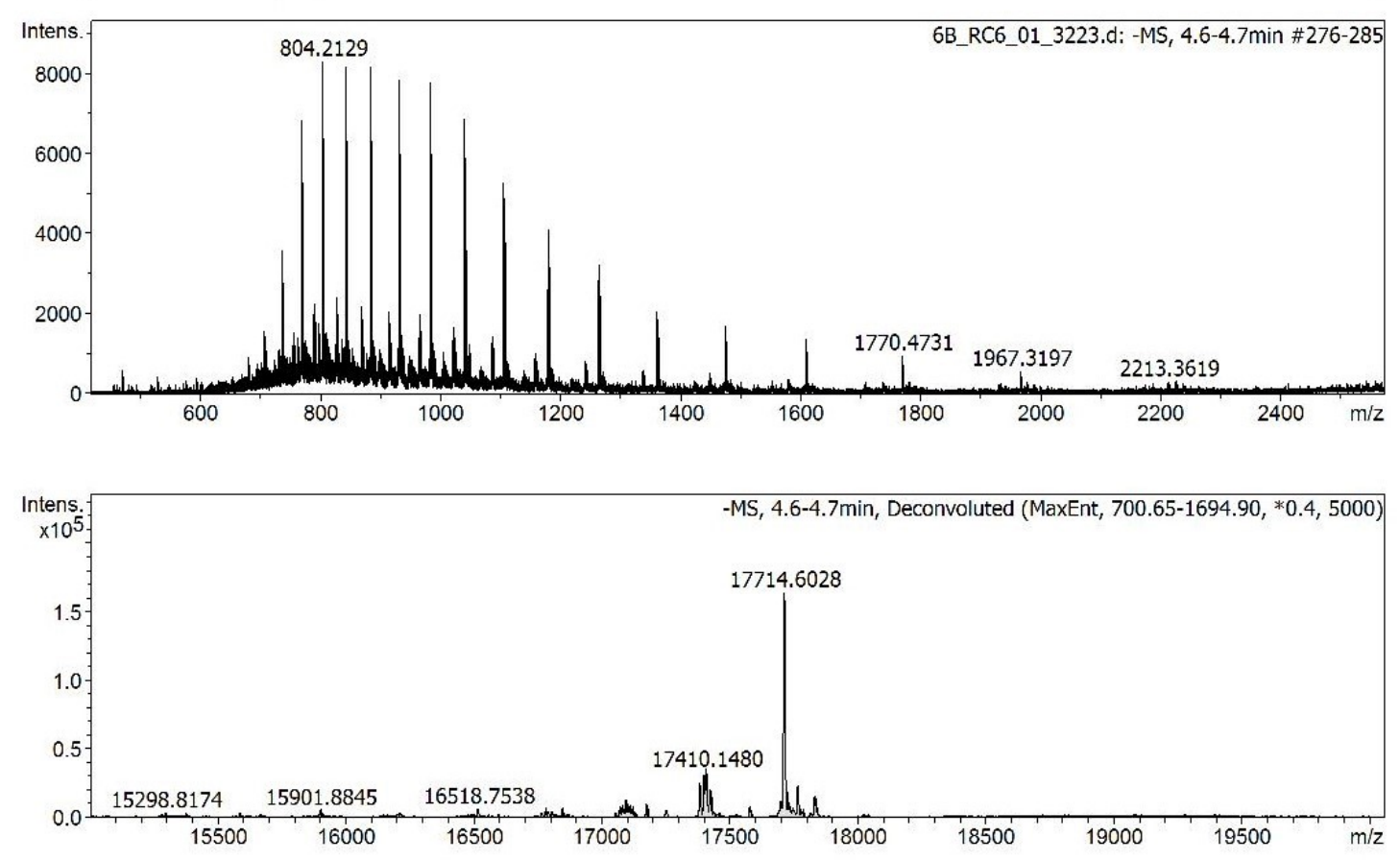

6B_RC6_01_3223.d

Bruker Compass DataAnalysis 4.1 $\quad$ printed: 11/14/2014 3:00:57 PM $\quad$ by: A Wahba $\quad$ Page 1 of 1


Mass spectrometric results for CCFL \#1 - Complete complement DNA (CC) sequences

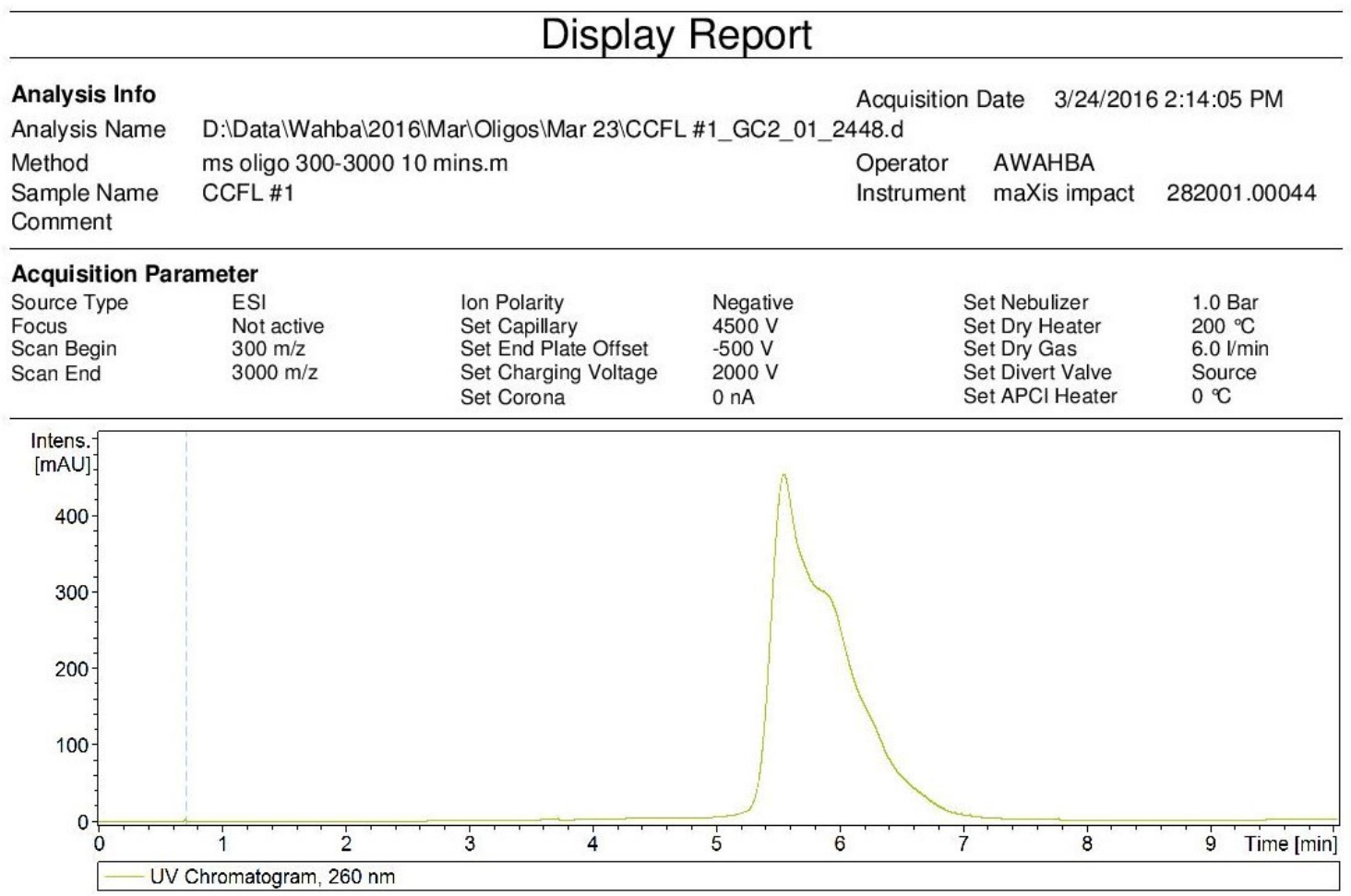
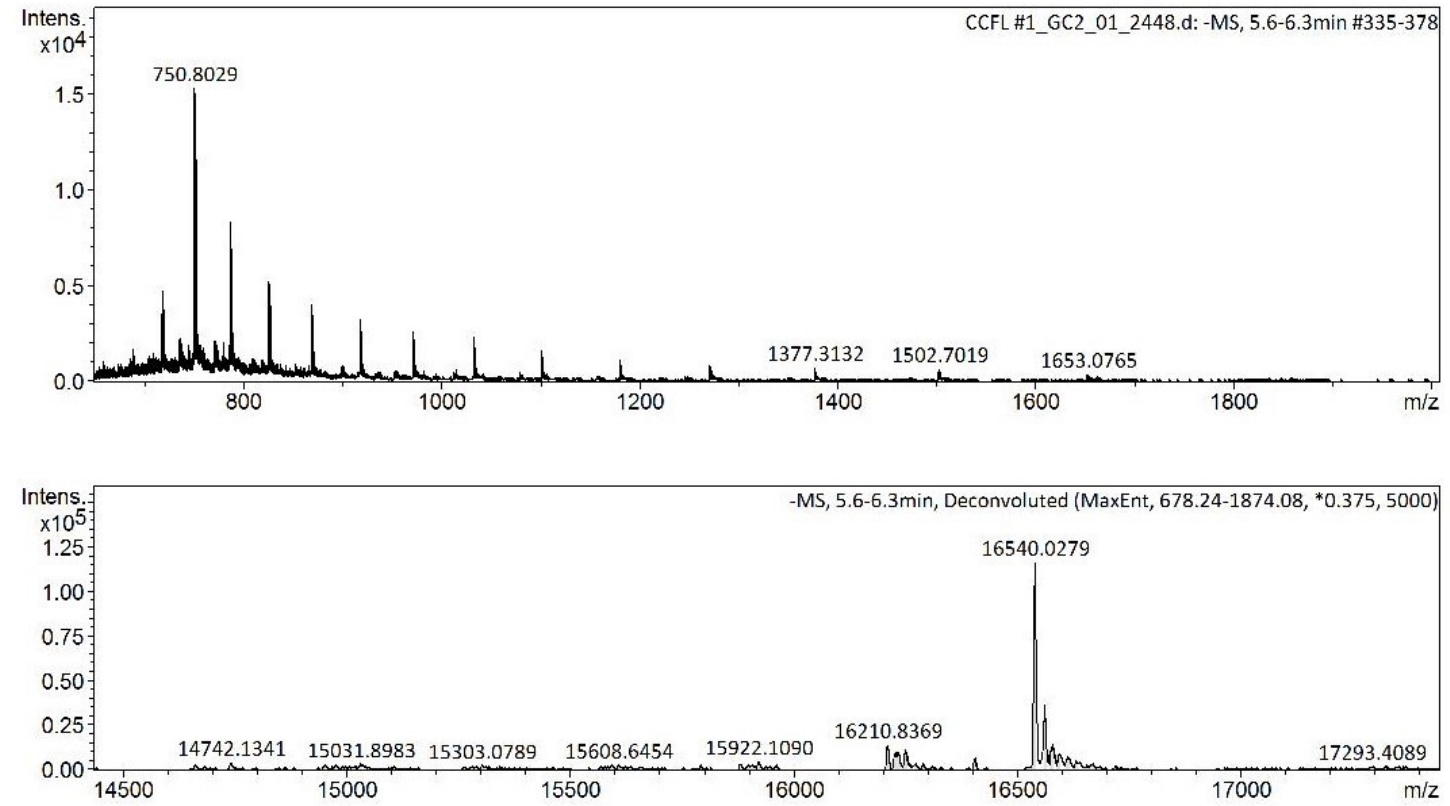

CCFL \#1_GC2_01_2448.d 
Mass spectrometric results for FL3 \#2 - Fluorescein labeled aptamer (FLX) sequences

Display Report

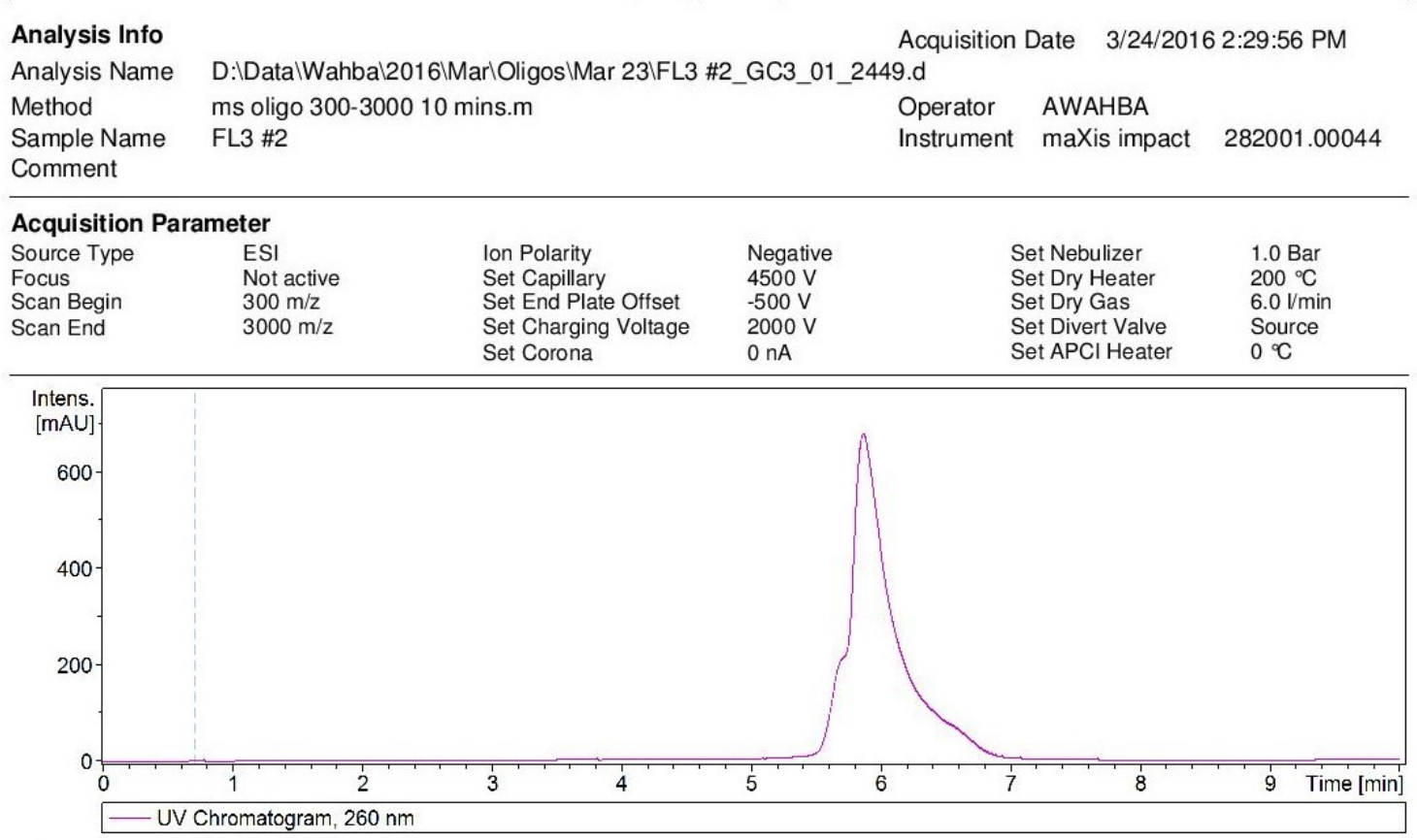
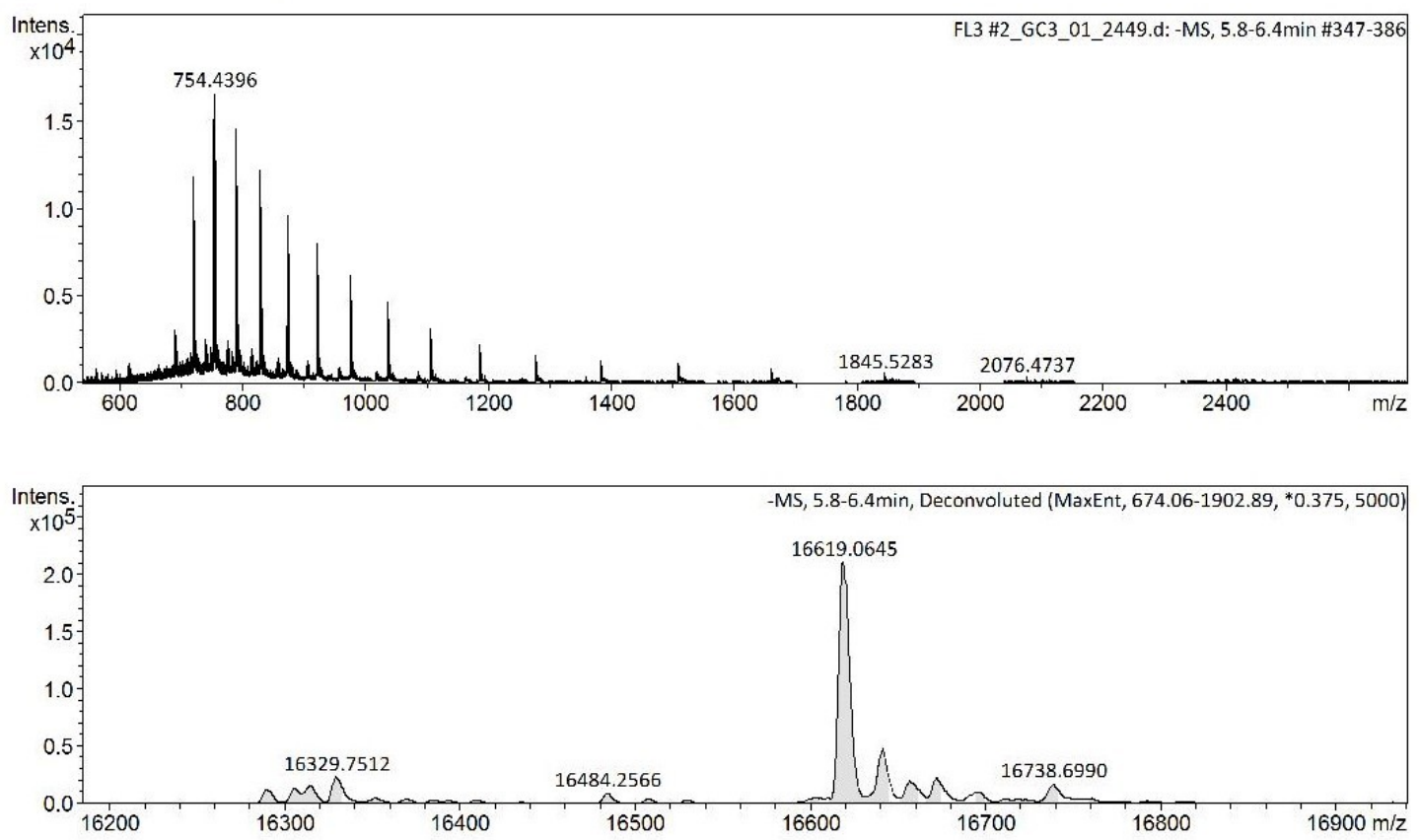

FL3 \#2_GC3_01_2449.d 
Mass spectrometric results for FLQ5 \#4 - fluorescein / dabcyl labeled aptamer beacon (FLQ) sequences

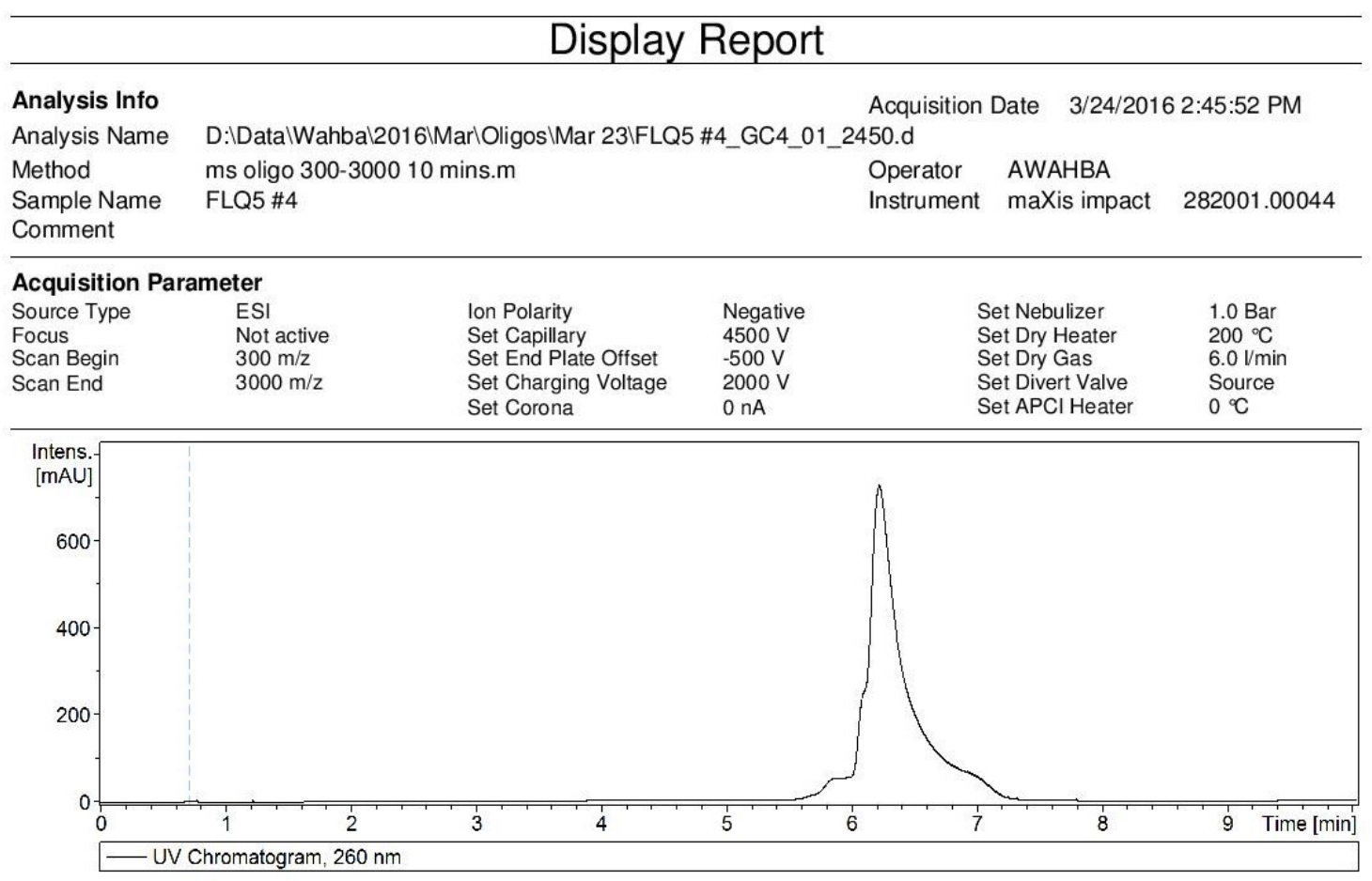
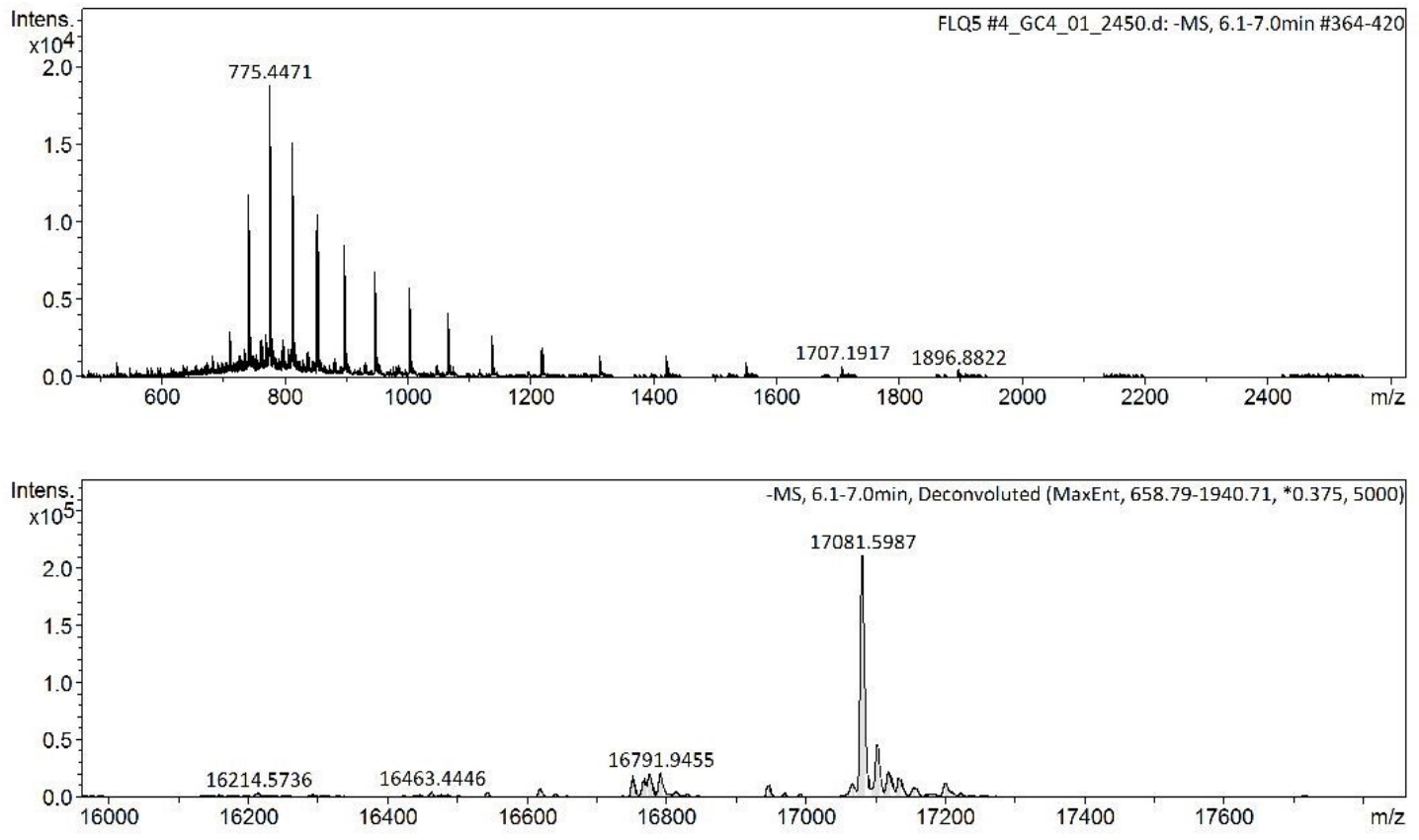

FLQ5 \#4_GC4 01 2450.d 